\author{
UNIVERSITY OF SÃO PAULO \\ SÃO CARLOS SCHOOL OF ENGINEERING \\ AND \\ CATHOLIC UNIVERSITY OF LEUVEN \\ FACULTY OF ENGINEERING SCIENCE
}

MARCELO LEITE RIBEIRO

Damage and progressive failure analysis for aeronautic composite structures with curvature

São Carlos

2013 

MARCELo LeITE Ribeiro

\section{Damage and Progressive Failure Analysis for Aeronautic Composite Structures with Curvature}

This thesis is submitted for the degree of Doctor of the University of São Paulo and KU Leuven in partial fulfillment of the requirements of the degree of Doctor in Mechanical Engineering.

Concentration Area: Aeronautical Engineering

Co-tutorship Thesis.

Brazil Supervisor: Associate Professor Volnei Tita (USP)

Belgium Supervisor: Professor Dirk Vandepitte (KU Leuven)

$$
\begin{gathered}
\text { ESTE EXEMPLAR TRATA-SEDA } \\
\text { VERSAO CORRIGIDA. } \\
\text { AVERSAOORIGINAL ENCONTRA-SE } \\
\text { DISPONIVEL WUTOAO } \\
\text { DEPARTAMENTO DEENGENHARIA } \\
\text { MECAMICA DA EESCUSP. }
\end{gathered}
$$

São Carlos

2013 
AUTORIZO A REPRODUÇÃO TOTAL OU PARCIAL DESTE TRABALHO POR QUALQUER MEIO CONVENCIONAL OU ELETROONICO, PARA FINS DE ESTUDO E PESQUISA, DESDE QUE CITADA A FONTE.

R314d Reiro, Marcelo Leite

Damage and progressive failure analysis for aeronautic composite structures with curvature / Marcelo Leite Ribeiro; orientador Volnei Tita. São Carlos, 2013.

Tese (Doutorado) - Programa de Pós-Graduação em Engenharia Mecânica e Área de Concentração em Aeronaves -- Escola de Engenharia de São Carlos da Universidade de São Paulo, 2013.

1. estruturas curvas. 2. materiais compósitos. 3. modelo de material. 4. análise progressiva de falhas. 5. elementos finitos. I. Título. 


\section{FOLHA DE JULGAMENTO}

Candidato: Engenheiro MARCELO LEITE RIBEIRO.

Título da tese: "Damage and progressive failure analysis for aeronautic composite structures with curvature".

Data da defesa: 03/04/2013

\section{Comissão Julgadora:}

Prof. Associado Volnei Tita (Orientador)

(Escola de Engenharia de São Carlos/EESC)

Prof. Dr. Dirk Vandepitte

(Katholieke Universiteit Leuven/Bélgica)

Prof. Dr. Stepan Vladimirovitch Lomov

(Katholieke Universiteit Leuven/Bélgica)

Prof. Associado Jonas de Carvalho

(Escola de Engenharia de São Carlos/EESC)

Prof. Titular Humberto Breves Coda

(Escola de Engenharia de São Carlos/EESC)

Prof. Dr. Mariano Eduardo Moreno

(Universidade Federal de São Carlos/UFSCar)

\section{Resultado:}

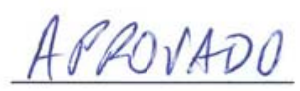

APPROVED .

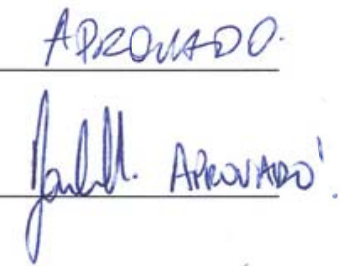

Aprovaclo

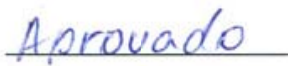

Coordenador do Programa de Pós-Graduação em Engenheira Mecânica:

Prof. Associado Marcelo Areias Trindade

Presidente da Comissão de Pós-Graduação:

Prof. Titular Denis Vinicius Coury 



\section{DEDICATION}

I would like to dedicate this work to my son Lucas, my wife Ana and my family for their support and love. 



\section{ACKNOWLEDGMENTS}

Several years of work have been spent to elaborate this thesis. Part of this time I was at KU Leuven Production Engineering, Machine Design and Automation (PMA) section and otheranother part I was at USP Aeronautical Engineering Department. In this meanwhile I have met many people who helped me to develop this thesis.

In special, I would like to thank my supervisors, Volnei Tita, for both his excellent tutorship and friendship and Dirk Vandepitte for the opportunity to work at KU Leuven as well as his suggestions for the development of this thesis.

I would like to thank my friends from KU Leuven, in special my friends who shared the office with me while I was in Belgium.

I also would like to thank my friends from USP in special those from the Aeronautical Structures Group.

A very special thank-you for my wife and son for their support and happy moments together. A special thank-you for the KU Leuven laboratory staff that helped me a lot to carry out the experiments. I also would like to thank the people from Aeronautical department for their help with several issues during this time.

At last, I would like to thank CAPES and Arenberg doctoral school for the financial support without which I would not be able to write this thesis. 

RIBEIRO, M. L. (2013) Modelos de falha e dano para estruturas aeronáuticas com curvatura e fabricadas em material compósito. 234p. Tese - Escola de Engenharia de São Carlos, Universidade de São Paulo, São Carlos.

As recentes melhorias nos processos de fabricação e nas propriedades dos materiais associadas a excelentes características mecânicas e baixo peso tornam os materiais compósitos muito atrativos para aplicação em estruturas aeronáuticas. No entanto, mesmo novos projetos, ainda são muito conservadores, pois os fenômenos de falha dos compósitos são muito complexos. Vários critérios e teorias de falha têm sido desenvolvidos para descrever o processo de dano e sua evolução, mas a solução do problema ainda está em aberto. Além disso, técnicas modernas de fabricação, como o enrolamento filamentar (filament winding) vêm sendo utilizadas para produzir uma ampla variedade de formas estruturais. Assim, este trabalho apresenta o desenvolvimento de um modelo de dano e a sua aplicação para simular a falha progressiva de estruturas planas e cilíndricas fabricadas em material compósito através do processo de filament winding. O modelo de dano proposto foi implementado como sub-rotinas em linguagem FORTRAN (UMAT-User Material Subroutine e, VUMAT-User Material Subroutine para simulações explícitas), que foram compiladas junto ao programa comercial de Elementos Finitos ABAQUS ${ }^{\mathrm{TM}}$. Várias análises numéricas foram realizadas via elementos finitos, a fim de prever a falha dessas estruturas de material compósito sob diferentes condições de carregamentos quase-estáticos e de impacto. Além disso, vários ensaios experimentais foram realizados, a fim de identificar os parâmetros relacionados com o modelo de material, bem como avaliar as potencialidades e as limitações do modelo proposto.

Palavras-chave: estruturas curvas, materiais compósitos, modelo de material, análise progressiva de falhas, Elementos Finitos. 

RIBEIRO, M. L.(2013) Damage and progressive failure analysis for aeronautic composite structures with curvature. 234p . PhD Thesis - São Carlos School of Engineering, University of São Paulo, São Carlos.

Recent improvements in manufacturing processes and materials properties associated with excellent mechanical characteristics and low weight have became composite materials very attractive for application on civil aircraft structures. However, even new designs are still very conservative, because the composite structure failure phenomena are very complex. Several failure criteria and theories have been developed to describe the damage process and how it evolves, but the solution of the problem is still open. Moreover, modern manufacturing processes, e.g. filament winding, have been used to produce a wide variety of structural shapes. Therefore, this work presents the development of a damage model and its application to simulate the progressive failure of flat composite laminates as well as for composite cylinders made by filament winding process. The proposed damage model has been implemented as a UMAT (User Material Subroutine) and VUMAT (User Material Subroutine for explicit simulations), which were linked to ABAQUS ${ }^{\mathrm{TM}}$ Finite Element (FE) commercial package. Progressive failure analyses have been carried out using FE Method in order to simulate the failure of filament wound composite structures under different quasi-static and impact loading conditions. In addition, experiments have been performed not only to identify parameters related to the material model but also to evaluate both the potentialities and the limitations of the proposed model.

Key-Words: curved structures, composite materials, material model, progressive failure analysis, Finite Element Method. 

Figure 1: (a) Boeing 787, (b) Airbus A380

Figure 2: (a) intra-ply failure of composite (Anderson, 1995); (b) inter-ply failure of composite (delamination)

Figure 3: (a) Lamina coordinate system, (b) Failure plane orientation (Puck e Schürmann, 2002)...41

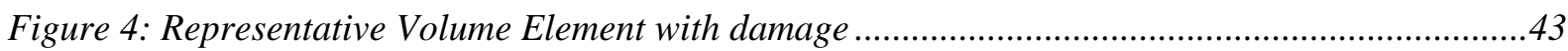

Figure 5: Experimental data (a); Secant modulus (b); Successive secant modulus (c).........................47

Figure 6: Stiffness degradation due to damage evolution. ………......................................................50

Figure 7: Damage threshold limit and experimental data ....................................................................51

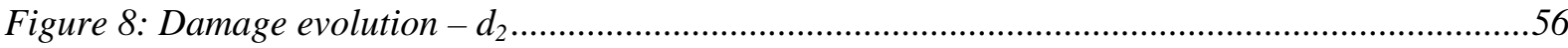

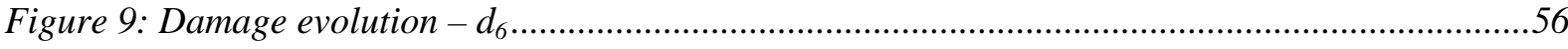

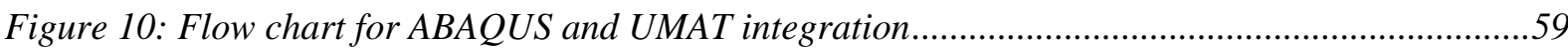

Figure 11: Vehicle impact simulation is an example of process that inertia effects are important.......59

Figure 12: Failure modes vs. orientation (Herakovich, 1998).............................................................72

Figure 13: (a) $0^{\circ}$ coupon dimensions; (b) $90^{\circ}$, coupons dimensions......................................................73

Figure 14: (a) Force vs. time history; (b) Strain vs. time history for a rosette strain gauge..................73

Figure 15: (a) Flat filament wound coupons; (b) Experimental test set-up at University of São Paulo

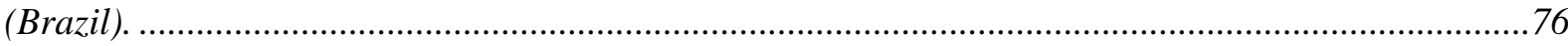

Figure 16: (a) Flat filament wound coupons; (b) Experimental test set-up at KU Leuven (Belgium)...76

Figure 17: (a) Coupon painted for image correlation procedure; (b) Image correlation strain field. .77

Figure 18: $5^{\circ}$ monotonic tensile test: Strain gages vs. Image correlation..........................................77

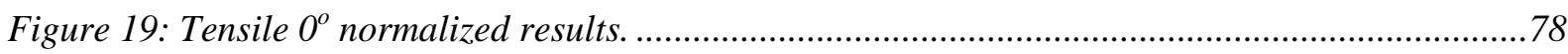

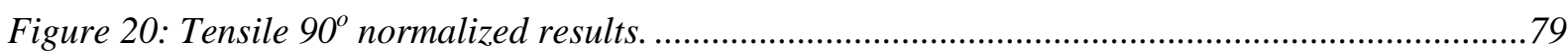

Figure 21: Applied loads in off-axis coupons during tensile tests using usual grips.............................84

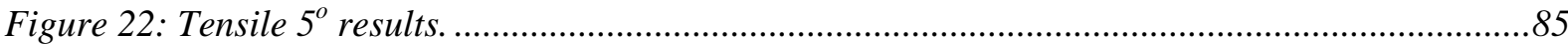

Figure 23: Comparison between experimental results and boundary conditions results for $5^{\circ}$............86

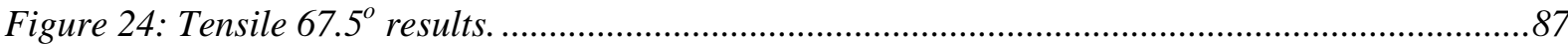

Figure 25: Acceptable and unacceptable failure modes for compression tests (ASTM D3410, 2003)..89

Figure 26: (a) Compression tests; (b) Compression coupons. ................................................................90

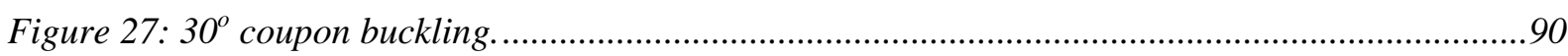

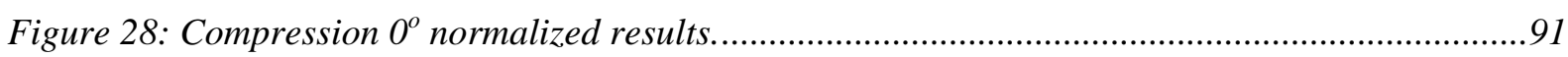

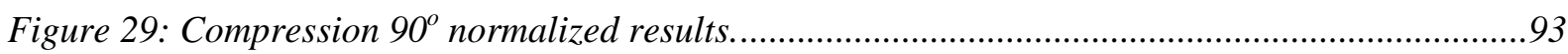

Figure 30: Comparison between experimental results and boundary conditions results for $15^{\circ}$..........94

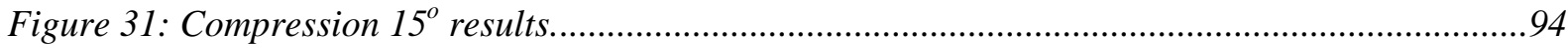


Figure 32: Comparison between experimental results and boundary conditions results for $30^{\circ} \ldots . . . . .95$

Figure 33: Compression $30^{\circ}$ results. 96

Figure 34: FE longitudinal displacement field for $50 \times 25$ mm gage length coupon (a) and $250 \times 25$ mm gage length coupon (b) - coupons with fibers at $\pm 45^{\circ}$...... 97

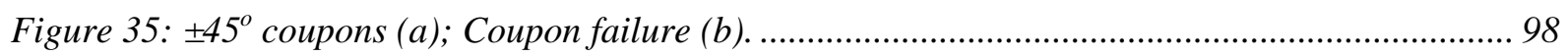

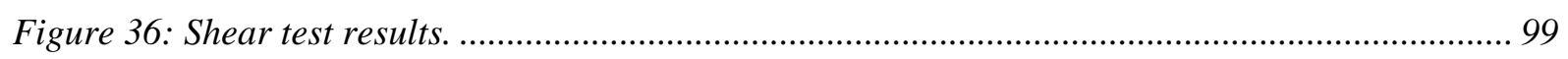

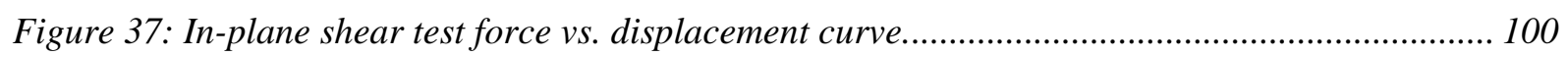

Figure 38: (a) Four point bending internal and external span dimensions. (b) Four point bending

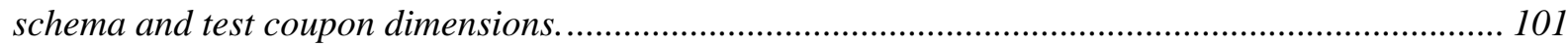

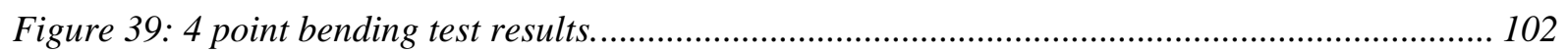

Figure 40: (a) Coupon dimensions; (b) Cylinder manufactured by filament winding process........... 105

Figure 41: (a) Drop tower apparatus, (b) Test coupon placed at the drop tower base (8.4 J tests); (c)

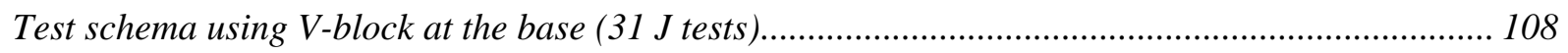

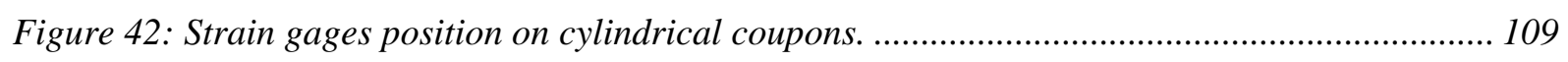

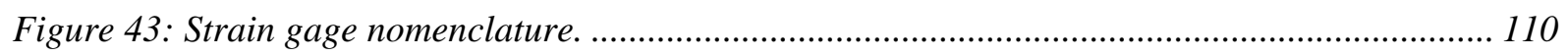

Figure 44: Type A (8.4 J) - force vs. time and displacement vs. time. …........................................... 111

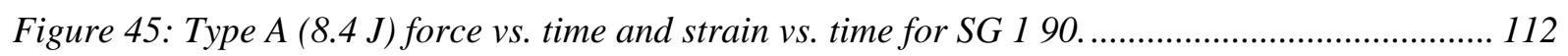

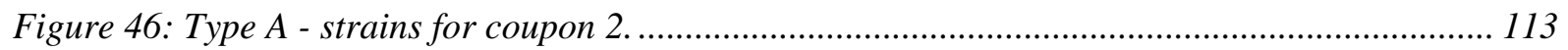

Figure 47: Kinetic energy balance between impactor and type A coupons for $8.4 \mathrm{~J}$ impact test. ....... 114

Figure 48: Type A (31 J) - force vs. time and displacement vs. time .............................................. 115

Figure 49: Kinetic energy balance between impactor and type A coupons for $31 \mathrm{~J}$ impact test. ....... 117

Figure 50: $31 \mathrm{~J}$ impacted area for type A cylinder. ......................................................................... 117

Figure 51: Type B cylinders (8.4 J impact energy): (a) C-Scan image; (b) force vs. time. ................. 119

Figure 52: Force vs. time history measured in the impactor and in the base for type B cylinders - 8.4J

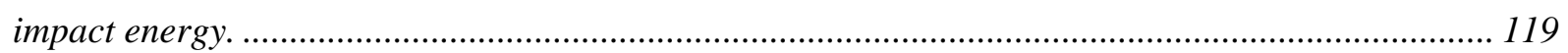

Figure 53: Type B (8.4 J) - force vs. time and displacement vs. time. .............................................. 121

Figure 54: Type B (8.4 J) - force vs. time and strain vs. time.......................................................... 121

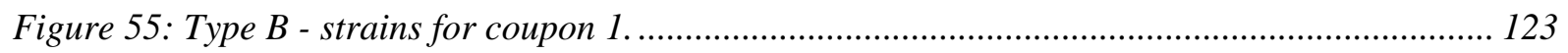

Figure 56: Kinetic energy balance between impactor and type B coupons for $8.4 \mathrm{~J}$ impact test. ...... 124

Figure 57: Type B (31 J) - force vs. time and displacement vs. time. ............................................... 125

Figure 58: Kinetic energy balance between impactor and type B coupons for $31 \mathrm{~J}$ impact test. ........ 126

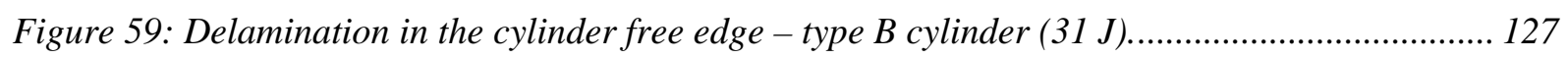

Figure 60: Type C (8.4 J) - force vs. time and displacement vs. time ................................................ 128

Figure 61: Type C (8.4 J) - force vs. time and strain vs. time.......................................................... 129

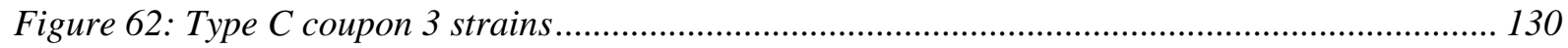

Figure 63: Kinetic energy balance between impactor and type C coupons for $8.4 \mathrm{~J}$ impact test....... 131

Figure 64: Type C (31 J) - force vs. time and displacement vs. time ................................................ 132 
Figure 65: Kinetic energy balance between impactor and type $C$ coupons for $31 \mathrm{~J}$ impact test.........133

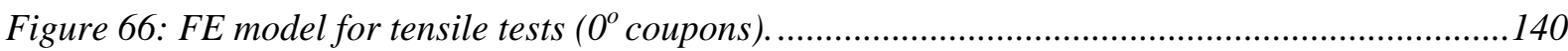

Figure 67: Tensile $0^{\circ}$ finite element mesh convergence analysis (a) and step size analysis (b).

Error $=\mid($ Experimental - Simulations $) /$ Experimental $\mid \%$

Figure 68: Normalized stress vs. strain for tensile $\left[0^{\circ}\right]_{10}$ results - numerical and experimental analyses.

Figure 69: Tensile $90^{\circ}$ finite element mesh convergence analysis (a) and step size analysis (b).

Error $=\mid($ Experimental - Simulations $) /$ Experimental $\mid \%$

Figure 70: Normalized stress vs. strain for tensile $\left[90^{\circ}\right]_{10}$ results - numerical and experimental analyses.

Figure 71: Tensile $5^{\circ}$ finite element mesh convergence analysis (a) and step size analysis (b).

Error $=\mid($ Experimental - Simulations $) /$ Experimental $\mid \%$

Figure 72: Stress vs. strain for tensile $\left[5^{\circ}\right]_{10}$ results - experimental vs. simulation.

Figure 73: Tensile $5^{\circ}$ coupon failure.

Figure 74: Compression $15^{\circ}$ finite element mesh convergence analysis (a) and step size analysis

Error $=\mid($ Experimental - Simulations $) /$ Experimental $\mid \%$ .148

Figure 75: Stress vs. strain for compression $\left[15^{\circ}\right]_{10}$ results - experimental vs. simulation. 148

Figure 76: Compression $15^{\circ}$ coupon failure. 149

Figure 77: Compression $30^{\circ}$ finite element (a) mesh convergence analysis and (b) step size analysis Error $=\mid($ Experimental - Simulations $) /$ Experimental $\mid \%$ 150

Figure 78: Stress vs. strain for compression $\left[30^{\circ}\right]_{10}$ results - experimental vs. simulation.

Figure 79: Four point bending finite element model ( $30^{\circ}$ coupons).

Figure 80: Off-axis $30^{\circ}$ 4-Point bending finite element (a) mesh convergence analysis and (b) step size analysis. Error $=\mid($ Experimental - Simulations $) /$ Experimental $\mid \%$ 153

Figure 81: Force vs. Displacement for four point bending $\left[30^{\circ}\right]_{10}$ results - numerical and experimental analyses.

Figure 82: Displacement field in z direction (blue arrow) and the bending-stretching coupling effect between the support and the coupon (in detail).

Figure 83: Stress fields just before the complete failure of the laminate: (a) Layer $1 \sigma_{11}$; (b) Layer 1

$\sigma_{22}$; (c) Layer $1 \tau_{12}$; (d) Layer $10 \sigma_{11}$; (b) Layer $10 \sigma_{22}$ and (c) Layer $10 \tau_{12}$. 156

Figure 84: Finite element model: (a) Cylinder geometry, boundary conditions and initial conditions for $30 \mathrm{~J}$ impact energy; (b) Impactor geometry, reference point, mass point and mesh. 158 Figure 85: Meshes for FE models. Three different meshes density used with structured square elements: coarse mesh (a); intermediate refined mesh (b); the most refined mesh (c). Free mesh algorithm with triangular elements (d) to verify the element type influence. 
Figure 86: Effect of mesh density and element type for type B cylinders........................................... 160

Figure 87: Hard contact (a) and exponential pressure-overclosure relationship (b)........................ 162

Figure 88: Type A contact parameters sensitivity study ..................................................................... 163

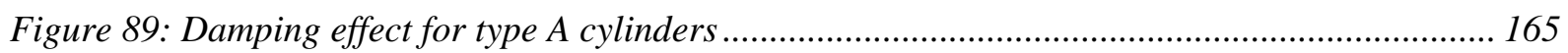

Figure 90: Mesh elements for investigation of the damping parameter influence on damage variables.

.

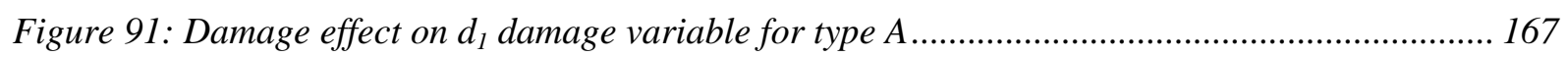

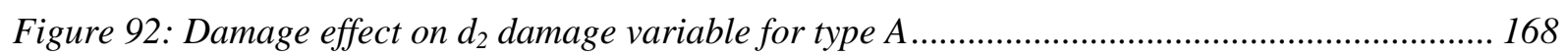

Figure 93: Damage effect on $d_{6}$ damage variable for type A............................................................... 169

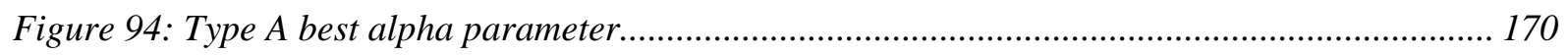

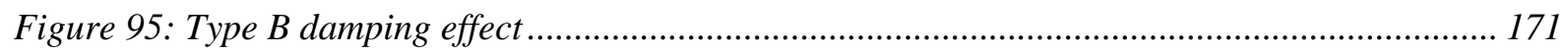

Figure 96: Damage effect on $d_{1}$ damage variable for type B........................................................... 172

Figure 97: Damage effect on $d_{2}$ damage variable for type $B$............................................................ 173

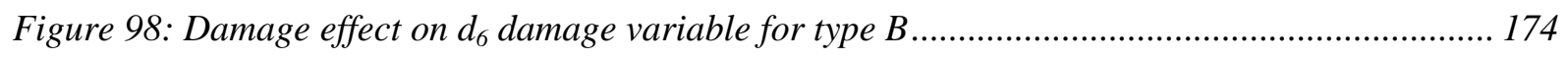

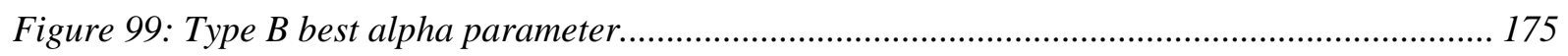

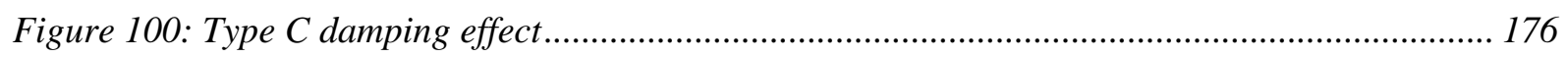

Figure 101: Damage effect on $d_{1}$ damage variable for type C...................................................... 177

Figure 102: Damage effect on $d_{2}$ damage variable for type $C$........................................................... 178

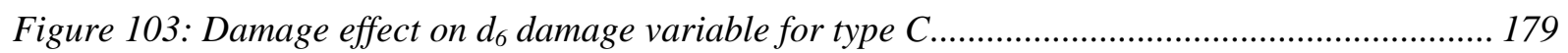

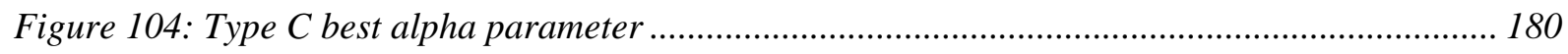

Figure 105: Type A cylinders: Simulation vs. Experimental results for (without damping effects).... 181

Figure 106: Type B cylinders: Simulation vs. Experimental results for (without damping effects).... 182

Figure 107: Type C cylinders: Simulation vs. Experimental results for (without damping effects)... 183

Figure 108: Type A simulation results. .......................................................................................... 184

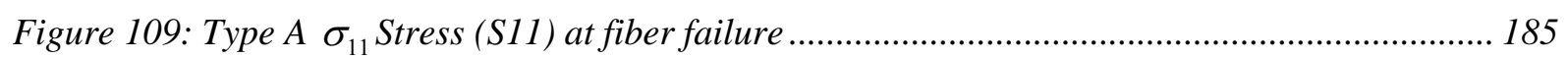

Figure 110: Type A $\sigma_{22}$ Stress (S22) at fiber failure .................................................................... 186

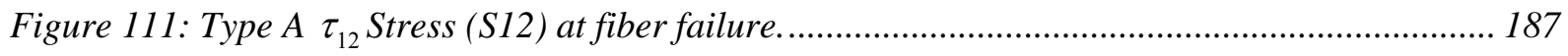

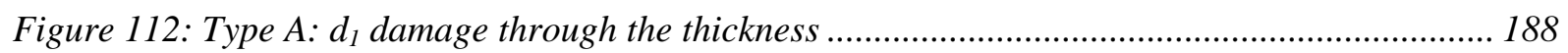

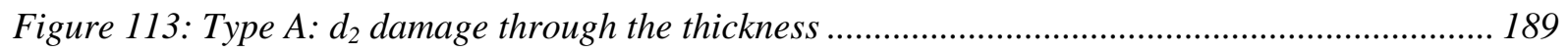

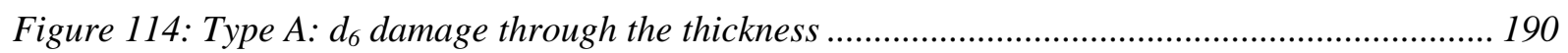

Figure 115: Damage variables for the impact point (total time) ......................................................... 191

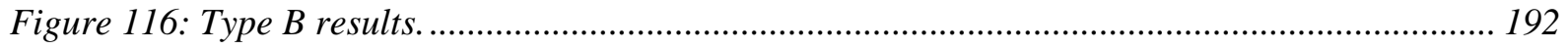

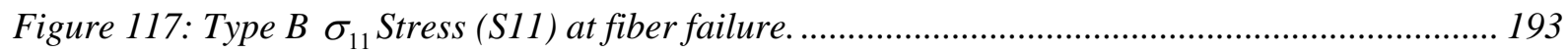

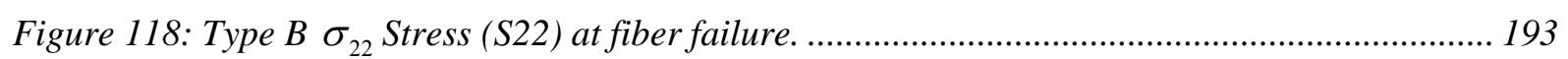

Figure 119: Type B $\tau_{12}$ Stress (S12) at the moment of layer 14 fiber breakage ................................... 194 
Figure 120: Type B: $d_{1}$ damage through the thickness ....................................................................195

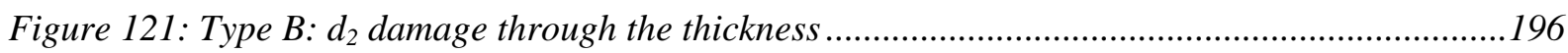

Figure 122: Type B: $d_{6}$ damage through the thickness ...................................................................197

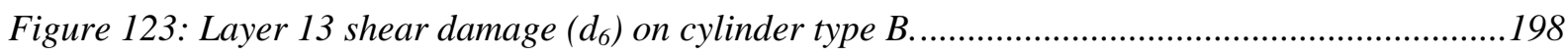

Figure 124: Type B damage variables through the thickness...........................................................198

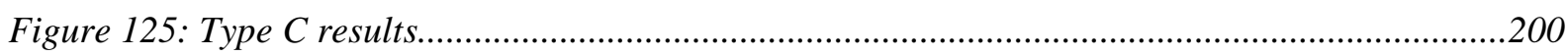

Figure 126: Type $C \sigma_{11}$ Stress (S11) at the moment of layer 14 fiber breakage.................................201

Figure 127: Type $C \sigma_{22}$ Stress (S22) at the moment of layer 14 fiber breakage ................................202

Figure 128 Type $C \tau_{12}$ Stress (S12) at the moment of layer 14 fiber breakage....................................203

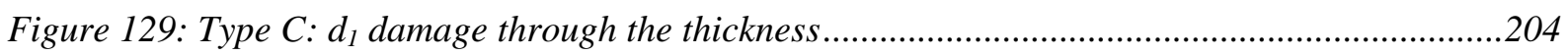

Figure 130: Type $C: d_{2}$ damage through the thickness ....................................................................205

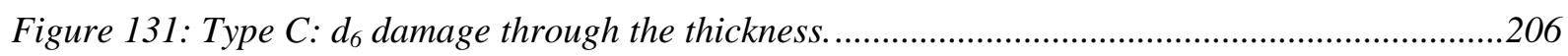

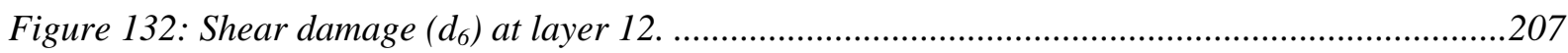

Figure 133: Type $C$ damage variables through thickness. ................................................................208 


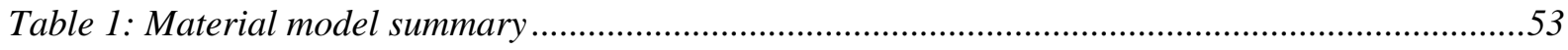

Table 2: Proposed model vs. Ladevèze ..............................................................................................54

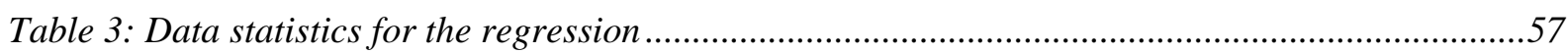

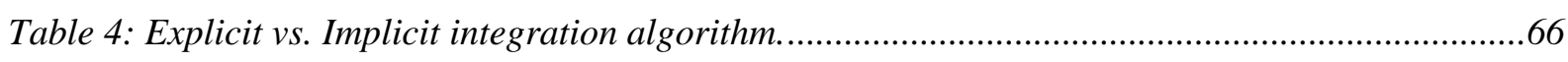

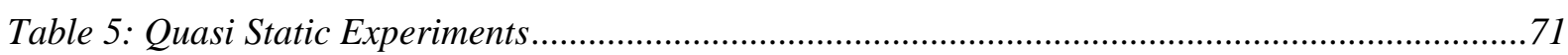

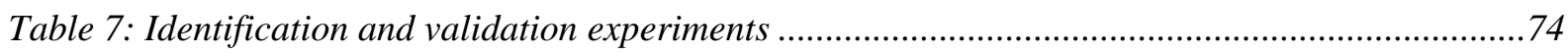

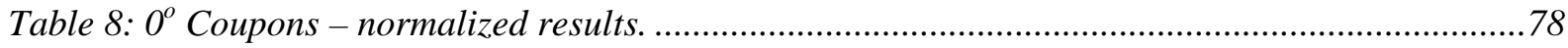

Table 9: Coupons with fibers at $90^{\circ}$ - normalized results. .................................................................79

Table 10: $0^{\circ}$ compression normalized tests results. ............................................................................92

Table 11: $90^{\circ}$ compression tests normalized results. .........................................................................93

Table 12: Shear elastic properties and strength values (Tita V. , 2003)..............................................98

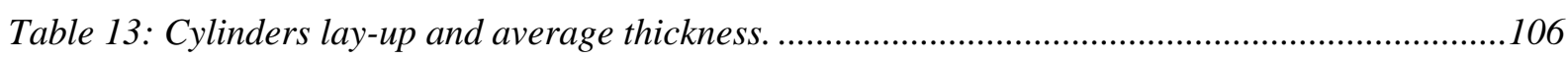

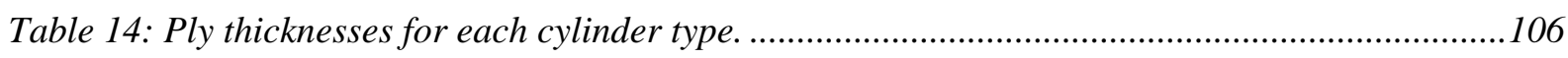

Table 15: Maximum force, displacement and strain for type A cylinders $(8.4 \mathrm{~J})$. ...............................112

Table 16: Ratio between elastic energy $\left(E_{e}\right)$ and dissipated energy $\left(E_{d}\right)-$ Type A cylinders ..............115

Table 17: Results summary for type A cylinders $(31 \mathrm{~J})$....................................................................118

Table 18: Ratio between elastic energy $\left(E_{e}\right)$ and dissipated energy $\left(E_{d}\right)$ - Type A cylinders $(31 \mathrm{~J}) \ldots .118$

Table 19: Maximum force, displacement and strain for type B cylinders $(8.4 \mathrm{~J})$. ...............................122

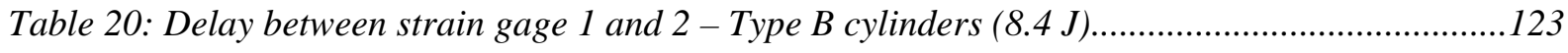

Table 21: Ratio between elastic energy $\left(E_{e}\right)$ and dissipated energy $\left(E_{d}\right)$ - Type B cylinders $(8.4 \mathrm{~J}) \ldots 124$

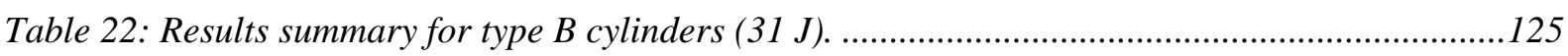

Table 23: Ratio between elastic energy $\left(E_{e}\right)$ and dissipated energy $\left(E_{d}\right)$ - Type B cylinders $(31 \mathrm{~J}) \ldots .127$

Table 24: Maximum force, displacement and strain for type $C$ cylinders $(8.4 \mathrm{~J})$................................129

Table 25: Delay between strain gage 1 and 2 - Type C cylinders $(8.4 \mathrm{~J})$.......................................130

Table 26: Ratio between elastic energy $\left(E_{a}\right)$ and dissipated energy $\left(E_{d}\right)$ - Type C cylinders (8.4 J) ..131

Table 27: Maximum force and displacement for type $C$ cylinders $(31 \mathrm{~J})$............................................132

Table 28: Ratio between elastic energy $\left(E_{a}\right)$ and dissipated energy $\left(E_{d}\right)$ - Type $C(31 \mathrm{~J})$..................133

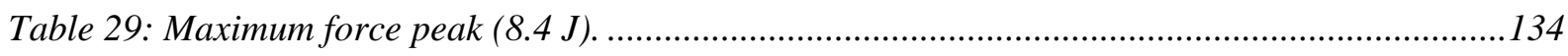

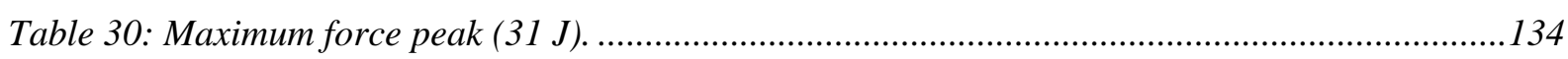

Table 31: Maximum displacement (absolute values) for $8.4 \mathrm{~J}$..........................................................135

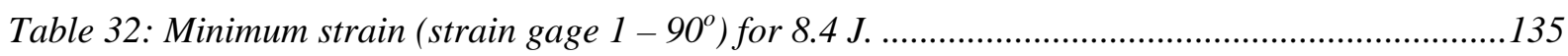

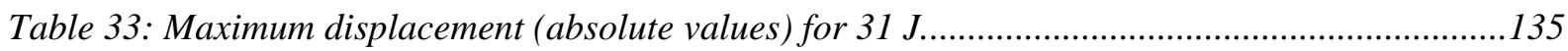

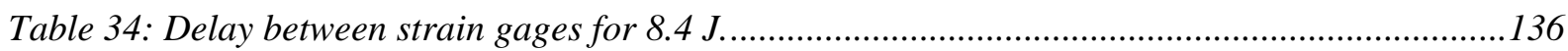




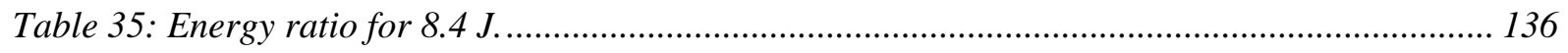

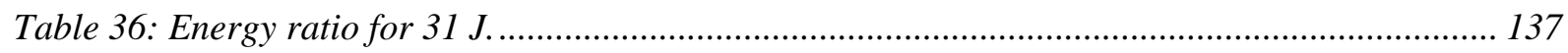

Table 37: Tensile $\left[0^{\circ}\right]_{10}$ normalized results for numerical and experimental analyses. ..................... 142

Table 38: Tensile $\left[90^{\circ}\right]_{10}$ normalized results for numerical and experimental analyses. ................... 144

Table 39: Tensile $\left[5^{\circ}\right]_{10}$ results for numerical and experimental analyses. ....................................... 147

Table 40: Compression $\left[15^{\circ}\right]_{10}$ results for numerical and experimental analyses............................. 149

Table 41: Compression $\left[30^{\circ}\right]_{10}$ results for numerical and experimental analyses............................. 151

Table 42: $\left[30^{\circ}\right]_{10}$ four point bending for numerical and experimental analyses................................. 155

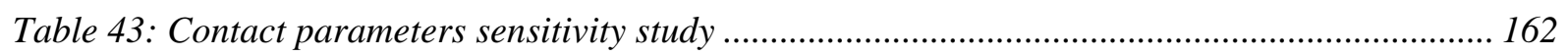

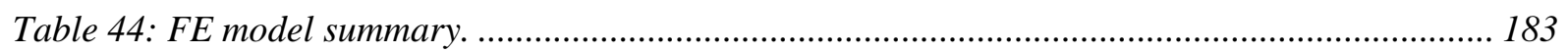




\begin{tabular}{|c|c|}
\hline ASTM & American standards for testing of materials \\
\hline UD & Unidirectional ply \\
\hline$V_{f}$ & Fiber volume fraction \\
\hline $\mathrm{V}_{\mathrm{m}}$ & Matrix volume fraction \\
\hline$d$ & Distance \\
\hline$D$ & Damage \\
\hline$D_{i j k l}$ & Forth order damage tensor \\
\hline$D_{\varepsilon}$ & Damage regarding the effective strain \\
\hline$d_{i}, i=1,2,6$ & $\begin{array}{l}\text { Damage variable (1- fiber direction, 2- } \\
\text { transverse direction, 6- ply plane/shear) }\end{array}$ \\
\hline$[C]$ & Damping matrix \\
\hline$E_{11}$ & Current elastic modulus in fiber direction \\
\hline$E_{11_{0}}$ & Original elastic modulus in fiber direction \\
\hline$E_{22}$ & $\begin{array}{l}\text { Current elastic modulus in transverse } \\
\text { direction }\end{array}$ \\
\hline$E_{22_{0}}$ & $\begin{array}{l}\text { Original elastic modulus in transverse } \\
\text { direction }\end{array}$ \\
\hline$E_{D}$ & Strain energy \\
\hline$E_{D C}$ & Strain energy critical value \\
\hline$f$ & Damage limit surface \\
\hline$\{F\}$ & Force vector \\
\hline FE & Finite Element \\
\hline$G_{12}$ & Shear modulus in ply plane \\
\hline$h$ & Coupon width \\
\hline$I_{i j k l}$ & Forth order identity tensor \\
\hline l & Coupon length \\
\hline$[K]$ & Stiffness matrix \\
\hline
\end{tabular}




\begin{tabular}{|c|c|}
\hline$[M]$ & Mass matrix \\
\hline$\vec{n}$ & Area normal vector \\
\hline$V_{i}$ & Initial speed \\
\hline$V_{f}$ & Final speed \\
\hline$U_{i}, i=x, y, z$ & Displacement in $\mathrm{x}, \mathrm{y}$ and $\mathrm{z}$ direction \\
\hline$\{u\},\{\dot{u}\},\{\ddot{u}\}$ & Displacement, speed and acceleration vectors \\
\hline$R_{i}, i=x, y, z$ & Rotation around $\mathrm{x}, \mathrm{y}$ and $\mathrm{z}$ axis \\
\hline $\bar{S}_{11}$ & Compliance matrix 11 term \\
\hline $\bar{S}_{16}$ & Compliance matrix 16 term \\
\hline $\bar{S}_{66}$ & Compliance matrix 66 term \\
\hline$S_{12}$ & Shear strength limit in ply plane \\
\hline$S_{12_{0}}$ & Shear linear elastic limit in ply plane \\
\hline$u$ & Displacement in $\mathrm{x}$ direction \\
\hline$\{u\}$ & Displacement vector \\
\hline$\{\dot{u}\}$ & Speed vector \\
\hline$\{\ddot{u}\}$ & Acceleration vector \\
\hline$\{\tilde{u}\}$ & Trial displacement vector \\
\hline$\{\dot{\tilde{u}}\}$ & Trial speed vector \\
\hline$V_{0}$ & Initial speed \\
\hline$X_{T}$ & Tensile strength limit in fiber direction \\
\hline$Y_{T}$ & Tensile strength limit in transverse direction \\
\hline$X_{C 0}$ & $\begin{array}{l}\text { Compression linear elastic limit in fiber } \\
\text { direction }\end{array}$ \\
\hline$X_{C}$ & Compression limit in fiber direction \\
\hline$Y_{C 0}$ & $\begin{array}{l}\text { Compression linear limit in transverse } \\
\text { direction }\end{array}$ \\
\hline$Y_{C}$ & Compression limit in transverse direction \\
\hline$\sigma_{i j}$ & Second order stress tensor \\
\hline
\end{tabular}




\begin{tabular}{|c|c|}
\hline$\hat{\sigma}_{i j}$ & Second order effective stress tensor \\
\hline$\sigma_{11}$ & Stress component in fiber direction \\
\hline$\sigma_{x}$ & $\begin{array}{l}\text { Stress component in principal material } \\
\text { orientation }\end{array}$ \\
\hline$\sigma_{22}$ & Stress component in transverse direction \\
\hline$\sigma_{22 y}$ & $\begin{array}{l}\text { Linear elastic limit for stress in transverse } \\
\text { direction }\end{array}$ \\
\hline$\sigma_{f}$ & $\begin{array}{l}\text { Acting stress in fiber direction at composite } \\
\text { failure }\end{array}$ \\
\hline$\sigma_{m}$ & $\begin{array}{l}\text { Acting stress in transverse direction at } \\
\text { composite failure }\end{array}$ \\
\hline$\sigma_{n}$ & Normal stress acting on fracture plane \\
\hline$\hat{\sigma}$ & Effective stress \\
\hline$\tau_{12}$ & Shear stress component in ply orientation \\
\hline$\tau_{x y}$ & Shear stress in principal material orientation \\
\hline$\tau_{n t}$ & $\begin{array}{l}\text { Normal-Transverse shear stress acting on } \\
\text { fracture plane }\end{array}$ \\
\hline$\tau_{n l}$ & $\begin{array}{l}\text { Normal-Longitudinal shear stress acting on } \\
\text { fracture plane }\end{array}$ \\
\hline$\varepsilon_{i j}$ & Second order strain tensor \\
\hline$\varepsilon_{11}$ & Strain component in fiber direction \\
\hline$\varepsilon_{x}^{0}$ & Middle plane strain in longitudinal direction \\
\hline$\varepsilon_{22}$ & Stress component in transverse direction \\
\hline$\varepsilon_{1 T}$ & Fracture tensile strain \\
\hline$\varepsilon_{f}^{*}$ & Fiber limit strain value \\
\hline$\varepsilon_{m}^{*}$ & Matrix limit strain value \\
\hline$\hat{\varepsilon}$ & Effective strain \\
\hline$\gamma_{12}$ & Shear strain component in ply plane \\
\hline$v_{12}$ & Major Poisson's coefficient \\
\hline$v_{21}$ & Minor Poisson's coefficient \\
\hline
\end{tabular}




$\begin{array}{cl}{[\phi]} & \text { Modal modes matrix } \\ \omega_{i} & \text { Natural frequency of the } i \text { mode } \\ \xi_{i} & \text { Damping factor } \\ \theta & \text { Ply orientation } \\ \psi & \text { Shape function } \\ \rho & \text { Density } \\ \lambda, \mu & \text { Lamé constants } \\ \Delta E_{C} & \text { Variation of kinetic energy } \\ \delta S & \text { Infinitesimal area } \\ \delta S_{D X} & \text { Infinitesimal damage area } \\ \partial \sigma / \partial \varepsilon & \text { Jacobian matrix }\end{array}$


TABLES XXI

SYMBOL XXIII

2.2 FaILURe MeChanisms AND Theoretical Models

2.2.1 LONGITUDINAL FAILURE

2.2.2 TRANSVERSE FaILURE

2.2.3 Continuous Damage Mechanics

2.3 Fiber Behavior Model

2.4 Damage Model for Transverse and Shear Loading 
2.6 Parameters Identification ANd Model Evaluation Experiments 55

2.7 Implementation VIa UMAT (USER MATERIal SUbroutine) 58

2.8 IMPACT SIMULATIONS

2.8.1 MASS MATRIX $\quad 60$

$\begin{array}{ll}\text { 2.8.2 DAMPING MATRIX } & 61\end{array}$

2.8.3 NUMERICAL SOLUTION: IMPLICIT VS. EXPLICIT 63

2.8.4 EXPLICIT SIMULATIONS VIA ABAQUS ${ }^{\text {TM }} 66$

$\begin{array}{llr}2.9 & \text { CONCLUSIONS } & 69\end{array}$

$\underline{3}$ EXPERIMENTS: FAILURE MECHANISMS

3.1 Quasi-STATIC TeSTS $\quad 71$

3.1.1 TENSILE TESTS $\quad 72$

$\begin{array}{lll}\text { 3.1.1.1 Materials and Methods } & 74\end{array}$

3.1.1.2 Results and Discussions 77

3.1.1.3 Tensile Tests Conclusions $\quad 87$

3.1.2 COMPRESSION TESTS 88

3.1.2.1 Materials and Methods $\quad 88$

3.1.2.2 Results and Discussions 90

3.1.2.3 Compression Tests Conclusions 96

$\begin{array}{lll}3.1 .3 & \text { SHEARTESTS } & 97\end{array}$

3.1.3.1 Materials and Methods 98

3.1.3.2 Results and Discussions 98

3.1.3.3 Shear Tests Conclusions 100

$\begin{array}{lll}\text { 3.1.4 BeNDING TESTS } & 100\end{array}$

3.1.4.1 Results and Discussions 102

3.1.4.2 Bending Tests Conclusions 102

$\begin{array}{lll}3.2 & \text { IMPACT TESTS } & 103\end{array}$

$\begin{array}{lll}3.2 .1 & \text { INTRODUCTION } & 103\end{array}$

$\begin{array}{ll}\text { 3.2.2 Materials and Methods } & 105\end{array}$

3.2.2.1 Cylindrical Test Coupons 105

3.2.2.2 Drop Tower Apparatus 107

3.2.3 EXPERIMENTAL ANALYSIS 110

3.2.3.1 Results for Cylinder Type A 111

$\begin{array}{lll}\text { 3.2.3.2 Results for Cylinder Type B } & 118\end{array}$ 
3.2.3.3 Results for Cylinder Type C

3.2.3.4 Comparison of Results 134

3.2.4 IMPACT TESTS CONCLUSIONS

4.1 Tensile Results

4.2 COMPRESSION RESULTS

4.3 Four-PoINT BENDING RESULtS

4.4 CONCLUSIONS FOR QUASI-STATIC TESTS AND SIMULATIONS

4.5 IMPACt ANALYSES

4.5.1 Mathematical Formulation 157

4.5.2 Finite Element Models 157

4.5.2.1 Mesh sensitivity studies 158

4.5.2.2 Contact algorithm studies 160

4.5.2.3 Damping coefficient studies 163

4.5.2.3.1 Type A damping effect. 164

4.5.2.3.2 Type B damping effect 170

4.5.2.3.3 Type C damping effect 175

4.5.3 RESULTS AND DISCUSSIONS 180

4.5.3.1 Damage vs. No damage. 180

4.5.3.2 Results

4.5.3.2.1 Results for Type A cylinders 183

4.5.3.2.2 Results for Type B cylinders 191

4.5.3.2.3 Results for Type C cylinders 199

4.5.4 IMPACT SIMULATION CONCLUSIONS 208 
In the last years, the use of composite materials for civil aircraft has increased for the development of primary structural elements. Some recent designs, e.g. Airbus A380 and Boeing 787 (Figure 1), use composite materials even for primary structural elements such as wing spars and fuselage skins, producing light weight structures without reducing the airworthiness. However, the application of composite materials in the civil aircraft structures is still limited due to both the difficulty in predicting their service life and the certification process ( Travessa (2006), Li and Goldberg (2011), Zhou and Gao (2012)). Also, the difficulty in predicting the structural failure modes (Puck \& Schürmann, 2002) requires better planned test program (Maimí, Camanho, Mayugo, \& Dávila, 2007) to reach the design requirements.

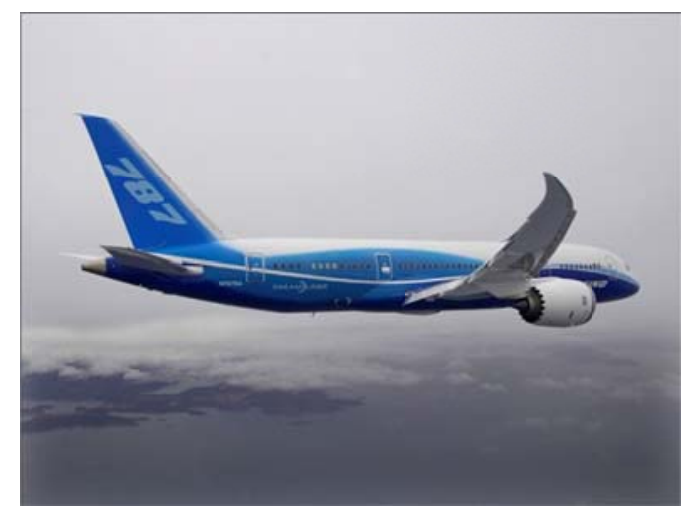

(a)

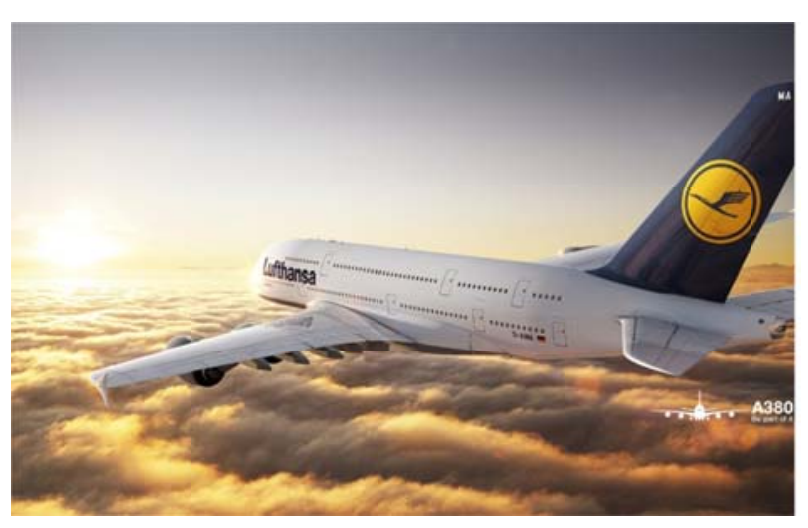

(b)

Figure 1: (a) Boeing 787, (b) Airbus A380

Among several causes of damage on composite structures, a special concern has been given to damage caused by impact loadings. In fact, composite structures are more susceptible to impact damage than the similar metallic structure. Impact loadings may cause internal damage in composite laminates, which cannot be detected normally by visual inspections (very small or internal damage), consisting of matrix cracking, delaminations and/or fiber breakage. However, this internal damage may cause a severe reduction of the structure strength (Abrate S. , 1998).

Associated to damage phenomena, the manufacturing process consists of another very important aspect for application of composite materials. Filament winding is one important 
method to produce composite parts. The basis of the filament winding process is the high speed and the precise deposition of continuous fibers impregnated by resin onto a mandrel. Several parts can be manufactured using a filament winding process, such as pressure vessels, pipes, drive shafts and aircraft structures (panels and flight command surfaces). These structures have high strength per weight, i.e. high specific strength. The geometry of the mandrel is limited to producing closed and convex structures. Filament winding can produce from small diameter tubes up to 40 meters, as well as large wind turbine blades. The manufacturing process is computer controlled and the reinforcement may be oriented to match the design requirement loads. With appropriate automation, this process is economically attractive (Callister, 2011) and, it is possible to produce a wide variety of structural shapes such as cylindrical parts and "quasi-flat" laminates (with high relative radius of curvature as $7.6 \mathrm{~m}$ ), which are manufactured by a special mandrel. However, it is worth to mention that complex parts even with cut-outs are not possible to be produced by using filament winding. In this case, it is more strategic to use, for example, fiber placement process, which produces aerospace parts with high quality.

In addition to the aspects commented above, it is very important to highlight that it is difficult to find scientific contributions in the literature for flat or quasi-flat composite laminates made by using filament winding. Thus, there is a scientific scenario, which motivates the development of a damage model and its application to simulate the progressive failure of quasi-flat (single curved) composite laminates and cylinders made by using filament winding process. Also, numerical analyses via FEM (Finite Element Method) to model the failure of flat filament wound composite laminates under different loading conditions are very attractive not only for providing scientific contributions, but also creating new technologies for the aeronautic industry.

\subsection{OBJECTIVES}

The present work aims to develop a material model to aid the study of damage and progressive failure analysis of unidirectional long fibers composite structures, mainly with curvature. This model should be easily implemented and would not significantly increase the computational cost of finite element analyses. Also, the model parameters must be easily identified and should require simple experiments. Therefore, the specific objectives of this work consist of: 
Formulating a new material model based on Continuum Damage Mechanics (CDM) in order to predict, with good accuracy, the behavior of composite structures (mainly with curvature) during failure process;

$>$ Implementing the material model proposal as a FORTRAN subroutine in order to be linked to the commercial finite element program ABAQUS ${ }^{\mathrm{TM}}$. Thus, user material subroutines defined as UMAT (for quasi-static simulations) and as VUMAT (for dynamic simulations) need to be developed;

Proposing a set of experiments to identify the model parameters. Thus, two groups of experiments will be proposed. One group to characterize the material and another to evaluate the damage evolution and how the stress state interacts with the failure process. Based on this set of experiments, it is possible not only to characterize the material, but also to determine the model parameters and partially evaluate the material model potentialities;

Performing a set of experiments in order to evaluate more deeply the potentialities and limitations of the material model proposal, considering different geometries, stacking sequences, type of loadings and etc;

Carrying out Finite Element Analyses in order to simulate the experiments. Based on the comparison between the numerical analyses and the experimental results, it is necessary to discuss the advantages and disadvantages of the FE models.

\subsection{ORganization OF CHAPTERS}

In order to help the readers, this Thesis is divided into 7 (seven) Chapters, which are summarized as follows:.

$>$ CHAPTER 1: the first chapter presents the introduction, motivation, objectives and the Thesis organization;

$>$ CHAPTER 2: the second chapter presents the development of a new damage model for composite materials. The specific literature review of progressive damage analysis of composite materials is shown, as well as the theory used to develop the damage model. After that, the procedures to identify the model parameters from experiments are described in details. The new damage model is implemented as a FORTRAN subroutine (UMAT and VUMAT - “user material”) linked to finite element software 
ABAQUS ${ }^{\mathrm{TM}}$. The differences between implicit and explicit algorithms are also discussed;

CHAPTER 3: in the third chapter, the experiments results used to characterize the material, as well as to identify the model parameters are described in details. The experimental results to evaluate the model have also been presented (quasi-static and impact tests). Procedures from American Standards for Testing Materials (ASTM) have been used for all conventional tests and consulted as a guide for non conventional tests. The results of experiments are discussed in details;

CHAPTER 4: in this chapter, the new damage model is evaluated for quasi-static loading cases of "flat" specimens, as well as for impact loadings on cylinders, veryfing the model performance to simulate real filament winding structures. In fact, potentialities and limitations of the new damage model are shown, considering the comparison between numerical and experimental results;

CHAPTER 5: in this chapter, the final conclusions based on the results of the previous chapters are presented, correlating the obtained results and the established objectives. Also, in the final of this chapter, it is possible to find a list of issues, which could be investigated in future works;

CHAPTER 6: this chapter shows the scientific publications developed by the author during the PhD work.

CHAPTER 7: the last chapter has all the references used to develop the present Thesis. 
This chapter presents the development of the material model based on Continuous Damage Mechanics in order to predict the failure behavior of composite structures. This material model needs to be developed considering some important aspects:

$\checkmark$ Requiring only simple tests for model parameters identification;

$\checkmark$ Requiring only simple test coupons to be manufactured;

$\checkmark$ Being simple to be implemented as a computational program;

$\checkmark$ Possessing low computational cost;

It is important to mention that this material model has to be applied for either flat or curved composite structures made of stacked plies with long unidirectional fibers.

\subsection{FAILURE CRITERIA AND DEgradATION LAWS}

Two approaches are usually applied for laminate failure analysis. The first one, defined as "first ply failure", regards that the whole laminate fails when a single layer does. This approach does not consider alternative load paths for the other laminate layers and, usually, provides very conservative results. Also, the first ply failure approach only needs a failure criterion. The other one, defined as "last ply failure", is more complex because it considers that the laminate fails only when its last ply does. The last ply failure approach requires a failure criterion and a degradation law to be applied for the material elastic properties. In this approach, the failed ply loads are redistributed to the other laminate plies.

On the other side, several macromechanical failure theories have been proposed for composite materials. Some of them are adaptations of the isotropic failure criterion, which accouts the anisotropy effects of composite materials (Daniel \& Ishai, 2006). As reported by Daniel and Ishai (2006), lamina failure theories are classified as:

- Limit or noninteractive theories: either the lamina stress or the strain state acting values (in local coordinate system) are compared to the corresponding stress or strains allowable values. There are no interactions between the stress and strain components;

- Interactive theories: all the stress components are included in one expression and there is no distinction among the failure modes; 
- Theories based on failure modes : different failure criteria are defined for each failure mode.

In fact, the prediction of mechanical behavior for composite structures is very complicated, because these structures exhibit multiple types of damage before total laminate rupture. The intrinsic heterogeneity of composite materials makes the failure process very complex and not well defined. This process involves a quite different number of phenomena, such as fiber fracture, fiber pull out, matrix cracking, fiber debonding, fiber kinking, interface cracks and fiber splitting, which can be defined as intra-ply failure modes. In addition, composite structures are normally made of the stacking of plies. And, it is very common to observe also delaminations between plies, which are defined as inter-ply failure modes. It is important to highlight that the difficulty in predicting the structural failure modes (Puck \& Schürmann, 2002) requires better planned design test program (Maimí, Camanho, Mayugo, \& Dávila, 2007) for aeronautic applications. Thus, considering this scenario, recent evolutions have brought significant improvements to composite materials properties, e.g. tensile strength and inter-laminar fracture toughness (Yokozeki, Ogasawara, \& Ishikawa, 2005). However, these new discoveries have not been enough to overcome the challenges related to the prediction of composite structures mechanical behavior. On one side, the evaluation of compression failure has still been considered essential for the safety design of composite structures because the compressive strength is often lower than $60 \%$ of tensile strength (Budiansky \& Fleck, 1993). On the other side, under flexural loads, the mechanical behavior of the composite structures can be driven by matrix properties, which are much lower when compared to fiber properties. In addition, matrix not only transfers the stresses to the fibers, but also protects the fibers and provides an alternative load path when a fiber fails (Reid \& Zhou, 2000). Therefore, there are many reasons to improve the prediction of the mechanical behavior of composite laminates made of polymer matrix.

Continuous Damage Mechanics (CDM) has been applied by several authors in order to model failure phenomena in composite materials. For example, Donadon et al. (Donadon, Frascino, Arbelo, \& Faria, 2009) have recently applied the CDM with crack smeared formulation to model progressive failure mechanisms in composite structures. Pavan et al. (2010) have used CDM in the development of a material model, which accounts the viscoelastic effects in the failure process of composites. Flatscher and Pettermann (2011) performed finite element analyses for open hole specimens subjected to uniaxial tensile loads, combining CDM and Plasticity Theory. Besides, multiscale approaches have been addressed in order to develop models based on micromechanics and mesomechanics of laminated 
composites, and these approaches have created a synergy between them. In this context, a semi-discrete and multiscale approach that called the computational damage micromodel for laminated composites has been presented by Lubineau and Ladeveze (2008). Transverse microcracking and microdelamination have been described through discrete cracks, for which minimum cracking surfaces have been introduced according to finite fracture mechanics. The cracked ply was assumed to be made of a "fiber-matrix material", whose homogenized behavior has been described through a continuum mechanics model. The authors implemented the model into ABAQUS ${ }^{\mathrm{TM}}$ through a User Material subroutine (UMAT) and discussed the advantages and limitations of the new proposed approach. In fact, not only the multiscale approaches for micromechanics integrated to mesomodel with CDM have been used to evaluate composite failure but also the combination of Fracture Mechanics Theory and CDM. Lubineau (2010) described a pyramidal scheme to formalize the imbrication of classical micromechanics based on discrete fracture mechanics coupled with damage mechanics. The researcher has applied the pyramidal approach to deduce homogenized law to be implemented into a commercial finite element software.

\subsection{Failure Mechanisms And Theoretical Models}

Due to both composite heterogeneity and anisotropy, it has been observed multiple mechanisms of damage before the total failure. Thus, failure of composite materials and structures are very complex and not well defined. However, as commented earlier, it is possible to summarize composite laminate failures in two types of modes:

1. Intra-ply failure modes: damage at fibers, polymeric matrix and/or interface between fibers and matrix (Figure 2(a));

2. Inter-ply failure modes: delaminations between plies (Figure 2(b)). 


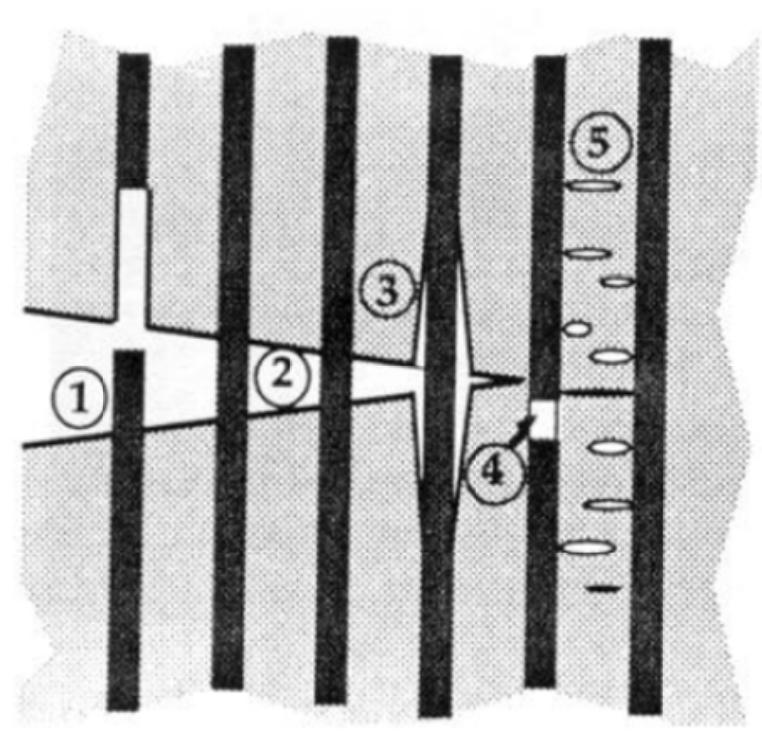

(a)

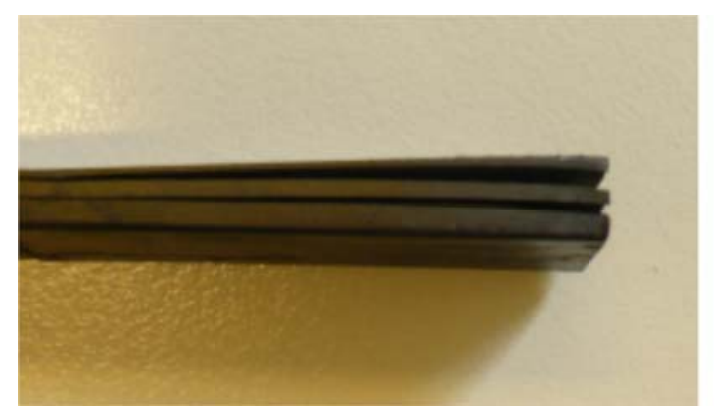

(b)

Figure 2: (a) intra-ply failure of composite (Anderson, 1995); (b) inter-ply failure of composite (delamination).

Mechanism 4, in Figure 2(a), is known as a fiber rupture. However, the fiber failure mode depends on the type of loading, for example, compression loads may induce micro-buckling, but tensile loads may induce rupture of fibers. The intra-ply damage at the matrix depends on the ductility of the polymer as well as on the in-service temperature. Thus, the polymeric matrix may present either a brittle or an inelastic behavior (mechanism 5). Mechanism 1, named "Pull-Out", occurs when the interface between fiber and matrix is weak. The fiber is pulled out of the matrix after the debonding mechanism (mechanism 3) takes place. If the interface between fiber and matrix is strong, the fiber will be not pulled out of the matrix, and the mechanism 2 named "Fiber Bridging" will be activated. The inter-ply failure, called delamination (Figure 2(b)), occurs after intra-ply damages, i.e., the evolution of intra-ply damages leads to delaminations, because the damaged regions grow as the load increases. Also, the cracks at two adjacent plies (with different orientation angles) join for creating a discrete failure between these layers. At that moment, the interlaminar shear increases strongly and the delamination process initiates. This failure mechanism is very common to occur under flexural and transversal shear stresses due to quasi-static or dynamic loading (Tita et al., 2008). It is important to highlight that under compression, laminate shows mostly elastic instability of the fibers (Puck e Schürmann, 2002). However, the compression failure mechanism is more complex and depending on the material properties. Hence, different compressive failure modes are possible to take place, e.g. microbuckling, fiber kinking and fiber failure (Pinho, 2005). 
Regarding the numerical methods used to simulate structures it is possible to mention that Finite Element Method (FEM) is the most popular numerical technique applied to structural analysis. The process analyses via FEM allow to model complex structures, providing displacements, strains and stresses components. Based on the stress components, it is possible to carry out the progressive failure analysis using a theoretical model and strength values as well. However, it is not a simple task, since some models demand a high computational effort whereas the analysis time may be considerable. Also, material with softening behavior normally presents severe convergence problems because the Jacobian matrix is no longer positive definitive. This issue is more pronounced when implicit finite elements programs are used (Lapczyk \& Hurtado, 2007). Nevertheless computational simulations create some problems, they can reduce the characterization costs of composite materials and support the optimization of these materials (Meer \& Sluys, 2009). Moreover, Xiao (2007) showed that some damage model parameters can be identified by correlations among computational simulations with standard material test results. In addition, some damage effects due to "free surfaces" and discontinuities can be detected by the reduction of some physical properties as stiffness, yield stress, hardness, ultrasonic wave velocity, density, etc (Lemaitre, 1996). Some of these physical effects allow measuring the damage in an inverse way, mainly with the support of CDM approach, which has been used by several authors to describe the damage process (initiation and propagation) as commented before.

Based on the considerations shown above, the present work used CDM to develop the mathematical formulation of the material model and numerical simulations via FEM in order to evaluate the potentialities of the proposed model.

\subsubsection{LONGITUdiNAL FAILURE}

When a unidirectional, UD, composite lamina is loaded in fiber direction (see Figure $3(\mathrm{a})$ ), the largest portion of the load is supported by the fibers due to their high stiffness compared to the matrix. Also the transmission of tensile loads in the fibers is not influenced by the state of damage in the matrix (Matzenmiller et al., 1995). The behavior of unidirectional lamina varies because of several factors such as: fiber volume fraction, matrix material, fiber material, manufacturing process, compressive or tensile load, etc. After fiber failure, the internal loads are redistributed and it may cause a structural collapse (Maimí, Camanho et al., 2007). 
In UD composites, intralaminar failure mechanisms trigger structural collapse almost immediately. However, multidirectional composites can support an increase of intralaminar failure before final collapse (Maimí, Camanho et al., 2007).

Considering that the fiber limit strain value is usually lower than the matrix limit strain value, when this unidirectional composite lamina is loaded in fiber direction, fibers will fail before the matrix. The majority of load supported by the fibers will be transferred to the matrix. However, under tensile loads, the fibers tend to straighten, what may contribute to matrix damage (Herakovich, 1998).

On the other hand, under compressive load, the composite failure is considered to be a fiber microbuckling problem. This phenomenon is influenced by several factors such as fiber size and shape, fiber waviness, fiber matrix bonding, fiber and matrix stiffness and strength (Herakovich, 1998). The compressive load carrying capacity is severely affected by the effective stiffness and strength of matrix. The matrix works as an elastic base for the fibers under compression (Matzenmiller, Lubliner et al., 1995).

Whereas fiber tensile strength $\mathrm{X}_{\mathrm{T}}$ can be regarded as the true fiber tensile strength, fiber compressive strength $\mathrm{X}_{\mathrm{C}}$ is usually not the true fiber compressive strength, because compressive failure mostly occurs through elastic instability (Puck e Schürmann, 2002). Also, compressive strength of composite materials is highly dependent on the fiber alignment, where low values of misalignment can lead to a drastic reduction of the compression strength ((Wisnom, 1990) (Yokozeki, Ogasawara et al., 2006)).

\subsubsection{TRANSVERSE FAILURE}

The transverse behavior of unidirectional composite (Figure 3(a) - directions 2 and 3) materials is highly anisotropic and for its strength it is considerable smaller comparing to fiber direction strength. Even when loaded in fiber direction, the composite may fail in transverse direction, having a significant influence for the composite strength (Callister, 2011). 


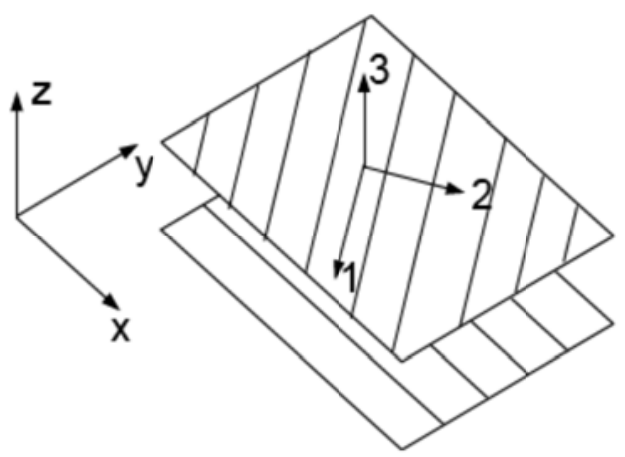

(a)

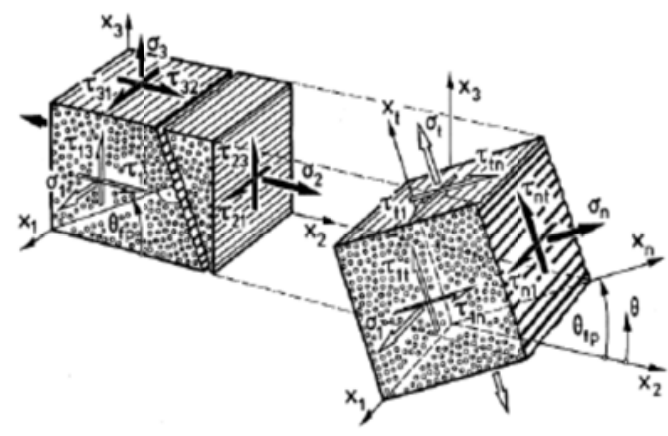

(b)

Figure 3: (a) Lamina coordinate system, (b) Failure plane orientation (Puck e Schürmann, 2002).

In the transverse direction, the normal and shear stresses are transmitted by both matrix and fibers. However, the damage occurs in the matrix and in the fiber-matrix interface. The bond strength between fiber and matrix is usually lower than the strength of each single constituent (Matzenmiller, Lubliner et al., 1995).

The failure in the transverse direction encompasses both matrix cracking and fiber-matrix debonding mechanism (Maimí, Camanho et al., 2007). Puck \& Schürmann (2002) assumed that UD carbon fiber-epoxy and glass fiber-epoxy composites behave in a very brittle way at failures, without previous apparent inelastic deformation. This brittle behavior can be better modeled by using Mohr failure criterion (Hashin, 1980).

The failure criterion developed by Puck \& Schürmann (1998) is based on physical considerations containing information about fracture angles. For plane stress under transverse tensile loading, $\sigma_{22}>0$, and in-plane shear stress, the existing defects in a ply (small debonds, voids, resin rich regions) are considered a trigger for a transverse crack, which extends through the ply thickness (Maimí, Camanho et al., 2007). These defects promote a non-linear behavior. Thus, the relation between shear stress and shear strain is non-linear before the failure. This behavior is also due to visco-plasticity of the matrix (Puck \& Schürmann, 2002). It is important to highlight that these transverse cracks do not affect the behavior of fibers.

Under compressive load, $\sigma_{22}<0$, matrix cracks crush in the sense of "fragmentation" of brittle matrix materials (Matzenmiller, Lubliner et al., 1995). If a compressive normal stress, $\sigma_{n}<0$, is acting on failure plane, the matrix failure is due to failure plane shear stresses, $\tau_{n l}$ and $\tau_{n t}$. For this case, this normal compressive stress prevents the shear fracture (Puck e Schürmann, 2002). Figure 3(b) shows the notations and coordinate system for unidirectional composite (UD) and the plane of fracture defined by Puck \& Schürmann (2002). 
Moreover, the cracks closures allow forces to be transmitted through the cracks, when $\sigma_{22}<0$. Schuecker \& Pettermann (2006) regarded this effect as stiffness recover for shear modulus.

Another important issue of composite transverse direction failure is how the shear stress affects the failure plane angle. For high in-plane shear stress compared to transverse stress, $\tau_{12}>\sigma_{22}$, the fracture plane is perpendicular to the mid-plane. And, increasing $\sigma_{22}$, the fracture plane angle changes (Maimí, Camanho et al., 2007).

\subsubsection{Continuous Damage MECHANics}

The qualitative mechanical behavior of engineering materials, such as metals, polymers, ceramics and composites, are very similar despite their physical differences. These similarities imply that common "mesoscopic" properties can be explained by energy mechanisms similar for all those materials. These characteristics make possible to apply the CDM and Thermodynamics Theories to model the material behavior, regardless the differences and complexity of their physical structure (Lemaitre, 1996).

The creation and growth of microvoids and microcracks are known as damage (Lemaitre and Desmorat, 2005). The deterioration of materials properties, when loaded, can be simulated through internal variables to describe the damage process (Lemaitre, 1996).

There are different forms of damage manifestations in a continuum media such as (Lemaitre, 1996):

$\checkmark$ Brittle damage occurs when a crack is initiated in a mesoscale without considerable plastic strains;

$\checkmark$ Ductile damage occurs when nucleation and growth of microvoids and microcracks take place in the media as a result of plastic strains (Kachanov, 1986);

$\checkmark$ Creep damage occurs when a metallic structure is loaded under elevated temperatures and the plastic strains involves viscosity (Lemaitre, 1996);

$\checkmark$ Low cycle fatigue damage occurs when a material is cyclic loaded at high values of stress or strain;

$\checkmark$ High cycle fatigue damage occurs under cyclic loads lower than a reference stress leading to a material deterioration. 
Kachanov (1986) presents other types of damage as embrittlement of steel, chemomechanical damage, environmental damage and damage of concrete, also.

The classical formulation of CDM regards the existence of energy potentials, whose the state law and constitutive equations are derived from. As mentioned before, damage is the creation of microvoids and microcracks (surface discontinuities); so regarding the smallest volume over which a measurement can be made (Representative Volume Element - RVE), it is possible to derive equations accounting the damage process (Figure 4).

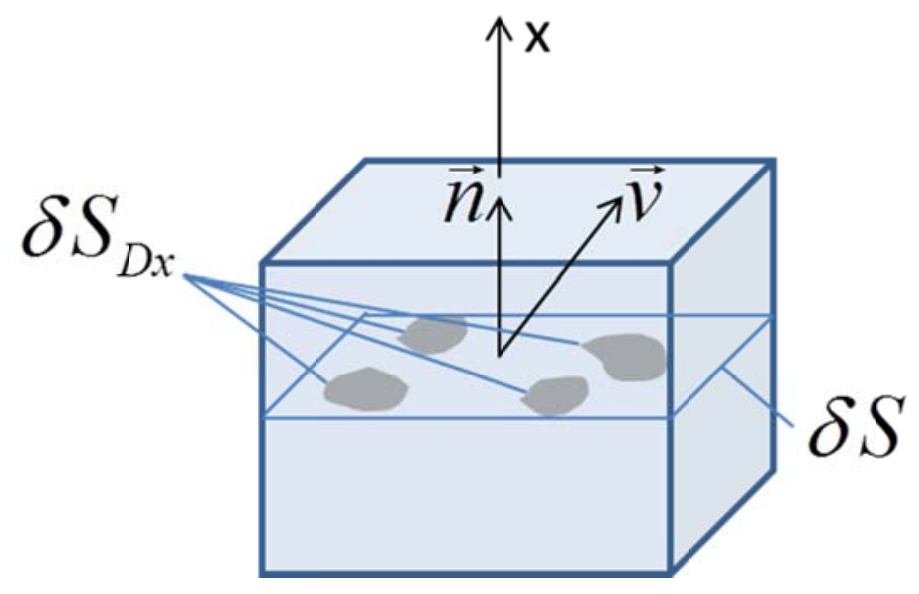

Figure 4: Representative Volume Element with damage

Where the damage of this element can be regarded as the ratio between the damage area ( $\left.\delta S_{D X}\right)$ and total area $(\delta S)$ :

$$
D(\vec{n}, x)=\frac{\delta S_{D X}}{\delta S}
$$

When $D=0$, the material is undamaged and when $D=1$, it is fully damaged (Lemaitre J. , 1996).

Damage usually is non-isotropic (Lemaitre \& Desmorat, 2005). Regarding a surface $\delta S$ with normal $\vec{n}$ and a reference vector $\vec{v}$ (Figure 4), a more general tensorial notation for damage is:

$$
\left(I_{i j k l}-D_{i j k l}\right) v_{k} n_{l} \delta S=v_{i} \hat{n}_{j} \delta \hat{S}
$$


Where $\hat{n}$ is the effective normal vector related with the effective area $(\delta \hat{S})$.

For anisotropic damage, a good representation of damage physics as well as its compatibility with thermodynamics is much more complicated. The effective stress is given by the following equation:

$$
v_{i} \hat{\sigma}_{i j} \hat{n}_{l} \delta \hat{S}=v_{i} \sigma_{k l} n_{j} \delta S
$$

Using the EQ. (2), it is possible to write:

$$
\hat{\sigma}_{i j}\left(I_{i j k l}-D_{i j k l}\right) v_{k} n_{l} \delta S=\sigma_{k l} v_{k} n_{l} \delta S
$$

It is important to mention that the fourth order damage tensor $\left(D_{i j k l}\right)$ has the following symmetries: $D_{i j k l}=D_{i j l k}=D_{j i k l}=D_{k l i j}$. Considering those symmetries, the effective stress tensor is (Lemaitre \& Desmorat, 2005):

$$
\hat{\sigma}_{i j}=\sigma_{k l}(I-D)_{k l i j}^{-1}
$$

Regarding the forth order damage tensor, there are some restrictions for this tensor:

$\checkmark$ Symmetry of the effective stress;

$\checkmark$ Effective stress independent of the strain behavior and Poisson's coefficient;

$\checkmark$ Compatibility with the Thermodynamics;

$\checkmark$ Different damage effect on the hydrostatic stresses behavior;

The actual elasticity tensor softened by damage is:

$$
\left(I_{i j r s}-D_{i j r s}\right) E_{r s k l}=\hat{E}_{i j k l}
$$

For composite materials, the damage mechanism is different in tension and shear. Thus, it is necessary two independent scalar variables to describe this influence on the elastic shear and hydrostatic energy. These variables are $D_{s}$ (for the deviator stress components) and $D_{n}$ 
(for hydrostatic stress). Hence, the anisotropic complementary energy density for damaged media is (Lemaitre J. , 1996):

$$
w_{e}=\frac{1}{2}\left(E_{i j k l}\right)^{-1} \sigma_{i j}^{D} \sigma_{k l}^{D}+\frac{1}{2}\left(E_{i j k l}\right)^{-1} \sigma_{H}^{2} \delta_{i j} \delta_{k l}
$$

Where $\sigma_{i j}^{D}$ is the stress deviator stress tensor, $\sigma_{H}$ is the hydrostatic stress and $\delta_{i j}$ is the Kronecker delta.

$$
\begin{gathered}
Y_{s}=\frac{\partial w_{e}}{\partial D_{s}} \\
Y_{n}=\frac{\partial w_{e}}{\partial D_{n}}
\end{gathered}
$$

These derivatives can be written, using engineering constants (Lemaitre J. , 1996):

$$
\begin{gathered}
Y_{s}=\frac{\partial w_{e}}{\partial D_{s}}=\frac{\tau_{12}^{2}}{2 G_{12}\left(1-D_{s}\right)^{2}} \\
Y_{n}=\frac{\partial w_{e}}{\partial D_{n}}=\frac{\sigma_{22}^{2}}{2 E_{22}\left(1-D_{n}\right)^{2}}
\end{gathered}
$$

Considering that the principal directions remain constant along the time, the damage has the same principal directions as the stress (Lemaitre J. , 1996). It results in effective stresses for three dimensional principal directions, which are:

$$
\hat{\sigma}=\left[\begin{array}{ccc}
\frac{\sigma_{11}}{\left(1-D_{1}\right)} & 0 & 0 \\
0 & \frac{\sigma_{22}}{\left(1-D_{2}\right)} & 0 \\
0 & 0 & \frac{\sigma_{33}}{\left(1-D_{3}\right)}
\end{array}\right]
$$


Where $D_{1}, D_{2}$ and $D_{3}$ are the damage variables in the principal directions for three dimensional stress states.

\subsection{Fiber BeHAVIOR MODEL}

For longitudinal tensile loads, i.e. loading applied in the fiber direction of the lamina $\sigma_{11}$, the behavior of each unidirectional lamina (e.g. made of carbon fiber with epoxy resin) under tensile can be considered to be linear elastic with a brittle fracture, which is simulated by using the Maximum Stress Criterion (EQ.(13)). After the failure detection, the damage variable in fiber direction, $\mathrm{d}_{1}$, is set to " 1 " (one).

There is no gradual evolution of the parameter $d_{1}$ in order to represent the abrupt failure phenomenon of the fibers, what has been observed during experiments. Therefore, $\mathrm{d}_{1}$ abrupt change may cause localizations issues. Thus, the degradation of properties occurs at the end of the time step via FEM solution. There is not any degradation during each iteration process. Hence, this strategy is similar to the viscous damage models, which create a time delay to reduce the properties by retarding the localization process. However, it is necessary to evaluate the time step in order to limit the size between the last step (where the damage was calculated) and the next step (where the damage is applied). Also, it is strategic to verify the FE mesh sensitivity during the numerical simulations in order to obtain better results.

$$
\frac{\sigma_{11}}{X_{T}} \geq 1
$$

On the other hand, the behavior of the composite lamina under compressive longitudinal loads is linear elastic until a specified value; then the lamina starts to behave like a nonlinear elastic material. This nonlinear elastic limit $X_{C 0}$ is identified by the experimental compression tests for $0^{\circ}$ coupons. And, the compression failure is detected by using the EQ.(14).

$$
\frac{\left|\sigma_{11}\right|}{X_{C 0}} \geq 1
$$


After $\left|\sigma_{11}\right| \geq X_{C 0}$, any increasing in the fiber compression load will result in a non-linear elastic stress-strain behavior. This non-linear elastic behavior is simulated using a secant modulus as shown by the EQ. (15):

$$
E_{11}=\frac{X_{C 0}}{\left|\varepsilon_{11}\right|}\left(1-h\left(\varepsilon_{11}\right)\right)+h\left(\varepsilon_{11}\right) E_{11_{0}}
$$

Where the parameter $h\left(\varepsilon_{11}\right)$ is obtained from the fit of stress-strain curves for $0^{\circ}$ coupons under compression loading; $\varepsilon_{11}$ is the strain component in the fiber direction and $E_{11_{0}}$ is the initial elastic modulus measured from experimental data of $0^{\circ}$ coupons under compression.

The procedure adopted in this work to fit the experimental curve is presented in Figure 5. For example, a hypothetical experimental curve shown in Figure 5(a) is required to be fit, by using the secant modulus.

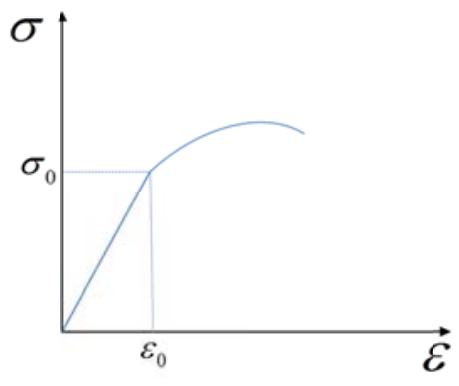

(a)

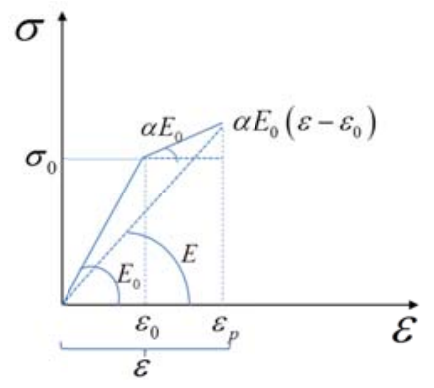

(b)

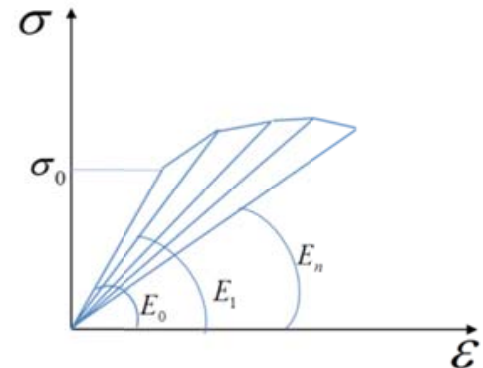

(c)

Figure 5: Hypothetical experimental data (a); Secant modulus (b); Successive secant modulus (c)

The linear elastic behavior of the stress strain curve is valid up to $\sigma_{0}$, after this stress level, the curve starts to show a nonlinear behavior. This nonlinearity can be modeled by using the secant method as shown by Figure $5(\mathrm{~b})$. In this figure, $E_{0}$ is the initial elastic modulus, $E$ is the secant modulus, $\alpha E_{0}$ is the stiffness for the stress-strain curve above the elastic limit $\sigma_{0}, \varepsilon$ is the total strain, $\varepsilon_{0}$ is the strain at $\sigma_{0}$ and $\varepsilon_{p}$ is the plastic strain. Based on Figure 5(b), the secant elastic modulus is given by: 


$$
E=\frac{\sigma_{0}}{\varepsilon}(1-\alpha)+\alpha E_{0}
$$

To improve the experimental curve fit, the previous procedure has been applied several times as shown by Figure 5(c). Thus the parameter $\alpha$ becomes function of the strain. For the present work, the parameter $h$ in EQ. (15) is the same as the parameter $\alpha$ in EQ. (16). Based on the real experimental data of $0^{\circ}$ compression curves, the linear regression for $h$ is given by EQ. (17). However, the experimental tests will be commented in details later.

$$
h\left(\varepsilon_{11}\right)=26.32 \cdot \varepsilon_{11}+0.35
$$

The previous approach to account for the effect of the compression loads on the structural behavior is different from the others shown by some researchers, who also used CDM in their model formulation.

\subsection{Damage Model For Transverse AND SHEAR LoAding}

Assuming a plane stress state in each unidirectional lamina of the quasi-flat filament wound laminate, the damage process in the matrix is driven by the stress components $\sigma_{22}$ (transverse loading - direction 2) and $\tau_{12}$ (shear loading - in plane 1-2). A nonlinear behavior has been observed in some experiments performed by the present author, mostly when the fibers and loading are not aligned. This nonlinear behavior is due to matrix inelastic strains and damage ( (Puck \& Schürmann, 2002), (Ribeiro, Tita, \& Vandepitte, 2012)). In order to model the matrix damage process, two internal damage variables $\mathrm{d}_{2}$ and $\mathrm{d}_{6}$ have been used, ranging from " 0 " (zero), for undamaged material, to " 1 " (one) for totally damaged material. Based on Continuous Damage Mechanics (CDM), the hypothesis of effective stress relates the damage variables to the plane stress state at the lamina (Herakovich, 1998) as shown by EQ. (18). 


$$
\left\{\begin{array}{l}
\hat{\sigma}_{11} \\
\hat{\sigma}_{22} \\
\hat{\tau}_{12}
\end{array}\right\}=\left[\begin{array}{ccc}
\frac{1}{1-d_{1}} & 0 & 0 \\
0 & \frac{1}{1-d_{2}} & 0 \\
0 & 0 & \frac{1}{1-d_{6}}
\end{array}\right] \cdot\left\{\begin{array}{c}
\sigma_{11} \\
\sigma_{22} \\
\tau_{12}
\end{array}\right\}
$$

EQ. (19) shows the damaged strain energy density in terms of effective stresses accounting only for matrix phase stresses (Herakovich, 1998).

$$
E_{D}=\frac{1}{2}\left[\frac{\left\langle\sigma_{22}^{2}\right\rangle_{+}}{E_{22_{0}}\left(1-d_{2}\right)}+\frac{\left\langle\sigma_{22}^{2}\right\rangle_{-}}{E_{22_{0}}}+\frac{\left|\tau_{12}^{2}\right|}{G_{12_{0}}\left(1-d_{6}\right)}\right]
$$

Where the operator $\langle x\rangle_{+}=x$, if $x>0$; otherwise $\langle x\rangle_{+}=0$, if $x<0$. And $\langle x\rangle_{-}=-x$, if $x<0$; otherwise $\langle x\rangle_{-}=0$, if $x>0$. Another important concept adopted consists of the Thermodynamic Forces, which relates the damage variables and strains energy density $E_{D}$ (EQ.(20) and (21)) (Ladeveze \& LeDantec, 1992).

$$
\begin{aligned}
& Y_{2}=\frac{\partial E_{D}}{\partial d_{2}}=\frac{\left\langle\sigma_{22}^{2}\right\rangle_{+}}{2 E_{22_{0}}\left(1-d_{2}\right)^{2}} \\
& Y_{6}=\frac{\partial E_{D}}{\partial d_{6}}=\frac{\tau_{12}^{2}}{2 G_{12_{0}}\left(1-d_{6}\right)^{2}}
\end{aligned}
$$

According to CDM, micro-cracks and micro-voids are opened in the matrix when the lamina is under transverse tensile stress, i.e. the load is applied in the direction normal to the fibers (direction 2). However, when the lamina is under transverse compression, micro-cracks and micro-voids can be closed in the matrix (Herakovich, 1998). To model this behavior, the damage variable $\mathrm{d}_{2}$ can change only when $\sigma_{22} \geq 0$, but the damage parameter $\mathrm{d}_{6}$ can change regardless of the shear stress sign, $\tau_{12}$.

The composite structures damage initiation can be identified by the stiffness reduction. The elastic properties degradation can be evaluated by performing a cyclic tensile or 
compression tests. During load-unload-load cycle is possible to observe this stiffness reduction compared to the initial stiffness (Figure 6).

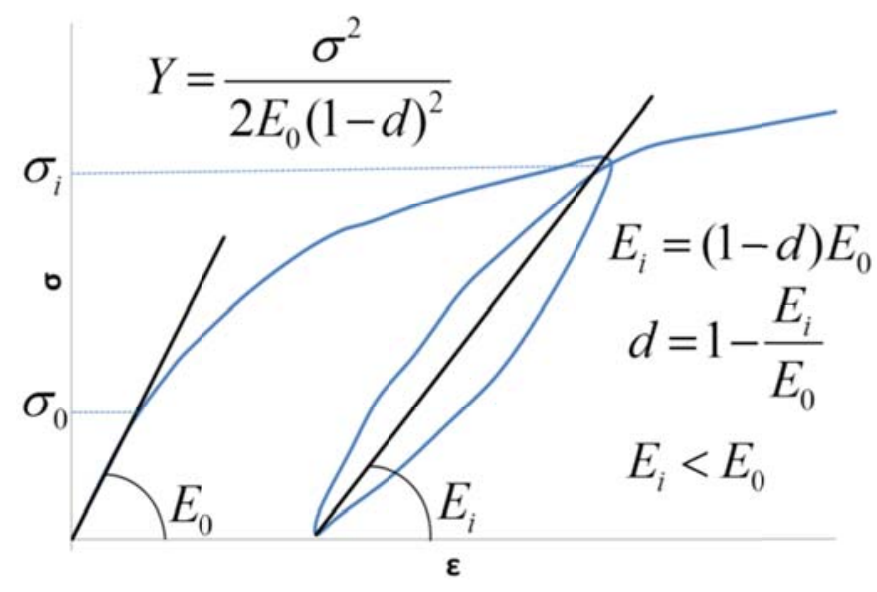

Figure 6: Stiffness degradation due to damage evolution.

To measure the damage, firstly, it is assumed that the structure is undamaged ( $d=0$ for $E_{0}$ ). Thus, the damage process is triggered when the structure starts to show a nonlinear behavior (response). On the damage process onset, the thermodynamic force is calculated regarding $d=0$. As the load increases, the first loading-unloading cycle shows the stiffness reduction. Thus the damage is calculated by using $d=1-E_{i} / E_{0}$ and the thermodynamic force is calculated by accounting the structural damage for the point, which corresponds to a loadingunloading cycle (Figure 6). In fact, this process has been repeated for each loading-unloading cycle.

Another important characteristic of the damage model is the mutual influence of $\sigma_{22}$ and $\tau_{12}$ in the matrix damage process as the damage measurement for off-axis coupon. Therefore, there are coupling parameters in order to calculate matrix damage process. Although these couple parameters were determined by Allix, Ladevèze, \& Vittecoq (1994) and Ladeveze \& LeDantec (1992), in this work, the material model regards that the damage variables $\mathrm{d}_{2}$ and $\mathrm{d}_{6}$ are written as a function of the ply orientation $(\theta)$ and the Thermodynamic Forces (Y). Also, these damage variables evolve following linear relations as shown by EQ. (22) and, the parameters $A(\theta), B(\theta), C(\theta)$ and $D(\theta)$ are identified through experiments.

$$
d_{2}=A(\theta) Y_{2}+B(\theta) ; \quad d_{6}=C(\theta) Y_{6}+D(\theta)
$$


The damage onset of composite laminates can be identified as the deterioration of materials properties (Kachanov, 1986). The elastic properties deterioration can be evaluated by quasi-static cyclic tensile and/or compressive tests. In this work, the damage onset surface is described by the EQ. (23), where $S_{12 y}$ is the shear stress linear elastic limit and $\sigma_{22_{0}}$ is the transverse linear elastic limit obtained from experimental analyses.

$$
f=\sqrt{\sigma_{22}^{2}+\tau_{12}^{2}}-\left[-S_{12 y}+\frac{2 S_{12 y}}{1+\left(\frac{\left|\sigma_{22}\right|}{\sigma_{22}}\right)^{3}}\right]
$$

Figure 7 shows the new damage onset surface and the experimental data values for each orientation. This new equation is used in this work and the experimental data showed a reasonable correlation with the surface equation. On the other hand, it is possible to observe that there are few experimental data and more tests could be conducted to verify the precision of the damage threshold surface.

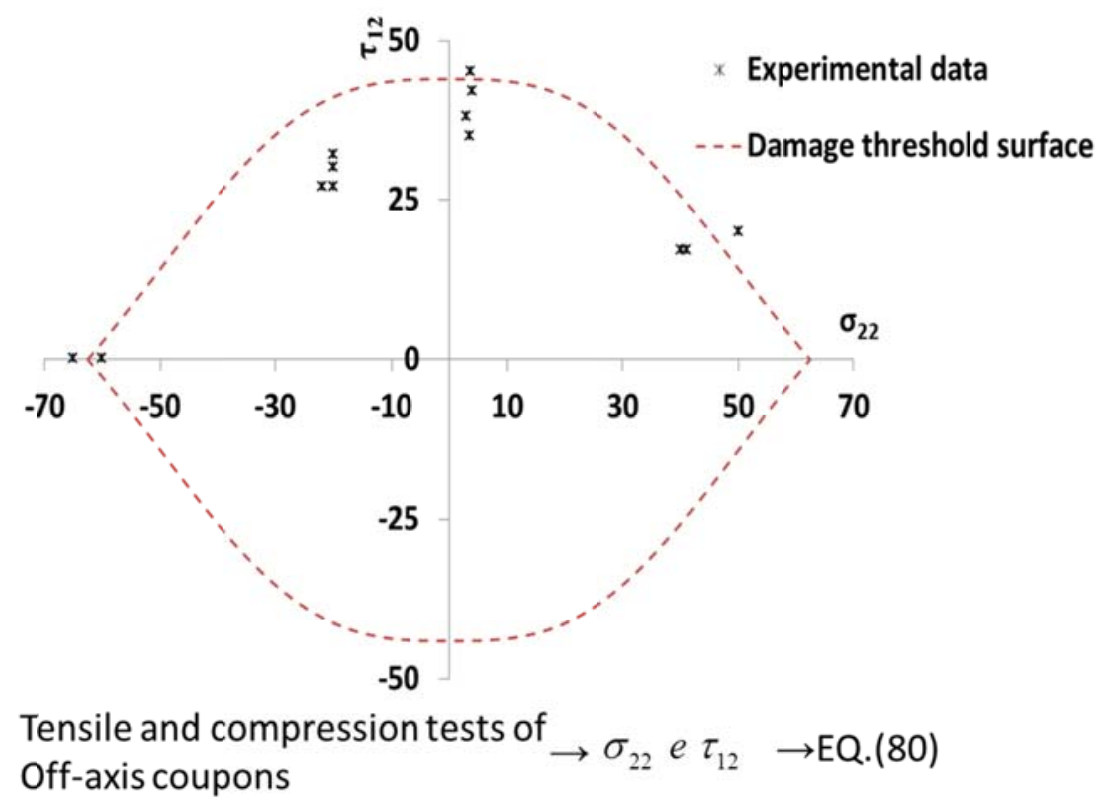

Figure 7: Damage threshold limit and experimental data

The damage onset occurs when $f \geq 0$ for a given stress state. Under transverse compressive loads $\sigma_{22}<0$, the matrix is regarded to have a nonlinear elastic behavior due to 
experimental evaluations (Ribeiro, Tita, \& Vandepitte, 2012). Therefore, this nonlinear behavior has been simulated by using a secant modulus (EQ. (24)).

$$
E_{22}=\frac{\sigma_{22 y}}{\varepsilon_{22}}\left(1-g\left(\varepsilon_{22}\right)\right)+g\left(\varepsilon_{22}\right) E_{22_{0}}
$$

The parameter $g\left(\varepsilon_{22}\right)$ is obtained from the fit of stress-strain curves for $90^{\circ}$ coupons under compressive loading. The procedure used for this case is similar to that used for fiber under compression. For the transverse compression, $\varepsilon_{22}$ is the strain component in transverse direction and $E_{22_{0}}$ is the initial elastic modulus measured from experimental data of $90^{\circ}$ coupons under compression.

Based on the real experimental data of $90^{\circ}$ compression curves, the linear regression is given by EQ. (25). However, the experimental tests will be commented in details later.

$$
g\left(\varepsilon_{22}\right)=14.61 \cdot \varepsilon_{22}+0.36
$$

This method to account the compression effects on the composite matrix is also different from those previously published by other authors. Besides, the material model considers the ply brittle fracture, using the strain energy, $E_{D}$, until this energy reaches a critical value. Thus, when $E_{D}$ is higher than a limit value $\left(E_{D C}\right)$ obtained by experiments, then $d_{2}$ is equal to "1" (one) and, $\mathrm{d}_{6}$ is equal to " 1 " (one), too.

\subsection{Material Model Summary}

In order to help the application of the material model, Table 1 summarizes the new damage model. 
Table 1: Material model summary

\begin{tabular}{ccc}
\hline Failure Criteria & Type of Failure & Degradation Law \\
\hline$\frac{\sigma_{11}}{X_{T}} \geq 1$ & Fiber Tensile & $E_{11}=0$ \\
\hline$\frac{\sigma_{11} \mid}{X_{C_{0}}} \geq 1$ & Fiber Compression & $E_{11}=\frac{X_{C_{0}}}{\left|\varepsilon_{11}\right|}\left(1-h\left(\varepsilon_{11}\right)\right)+h\left(\varepsilon_{11}\right) E_{11_{0}}$ \\
\hline$f \geq 0$ & Matrix Tensile & $d_{2}=A(\theta) Y_{2}+B(\theta)$ \\
\hline$f \geq 0$ & Matrix Compression & $E_{22}=\frac{\sigma_{22 y}}{\left|\varepsilon_{22}\right|}\left(1-g\left(\varepsilon_{22}\right)\right)+g\left(\varepsilon_{22}\right) E_{22_{0}}$ \\
\hline$f \geq 0$ & Shear & $d_{6}=C(\theta) Y_{6}+D(\theta)$ \\
\hline
\end{tabular}

Another important aspect of the damage model proposal consists of the adjustments for the Poisson's coefficients to take into account the damage effect. Using CDM formulation (Matzenmiller, Lubliner, \& Taylor, 1995), the compliance tensor is given by EQ. (23), where $K=\left(1-\left(1-d_{1}\right)\left(1-d_{2}\right) v_{12} v_{21}\right)$.

$$
D=\frac{1}{K}\left[\begin{array}{ccc}
\left(1-d_{1}\right) E_{11} & \left(1-d_{1}\right)\left(1-d_{2}\right) v_{21} E_{22} & 0 \\
\left(1-d_{1}\right)\left(1-d_{2}\right) v_{12} E_{11} & \left(1-d_{2}\right) E_{22} & 0 \\
0 & 0 & K\left(1-d_{6}\right) G_{12}
\end{array}\right]
$$

Finally, in order to exclude material self-healing behavior, the damage parameters $d_{1}, d_{2}$ and $\mathrm{d}_{6}$ never decrease from their maximum values obtained during the calculation process.

Table 2 presents the differences between the proposed damage model and Ladevèze's model (Ladeveze \& LeDantec, 1992). It is important to light that both models use the CDM framework to predict the material behavior, but it is very easy to identify the differences between some failure criteria and evolution laws. 
Table 2: Proposed model vs. Ladevèze's model

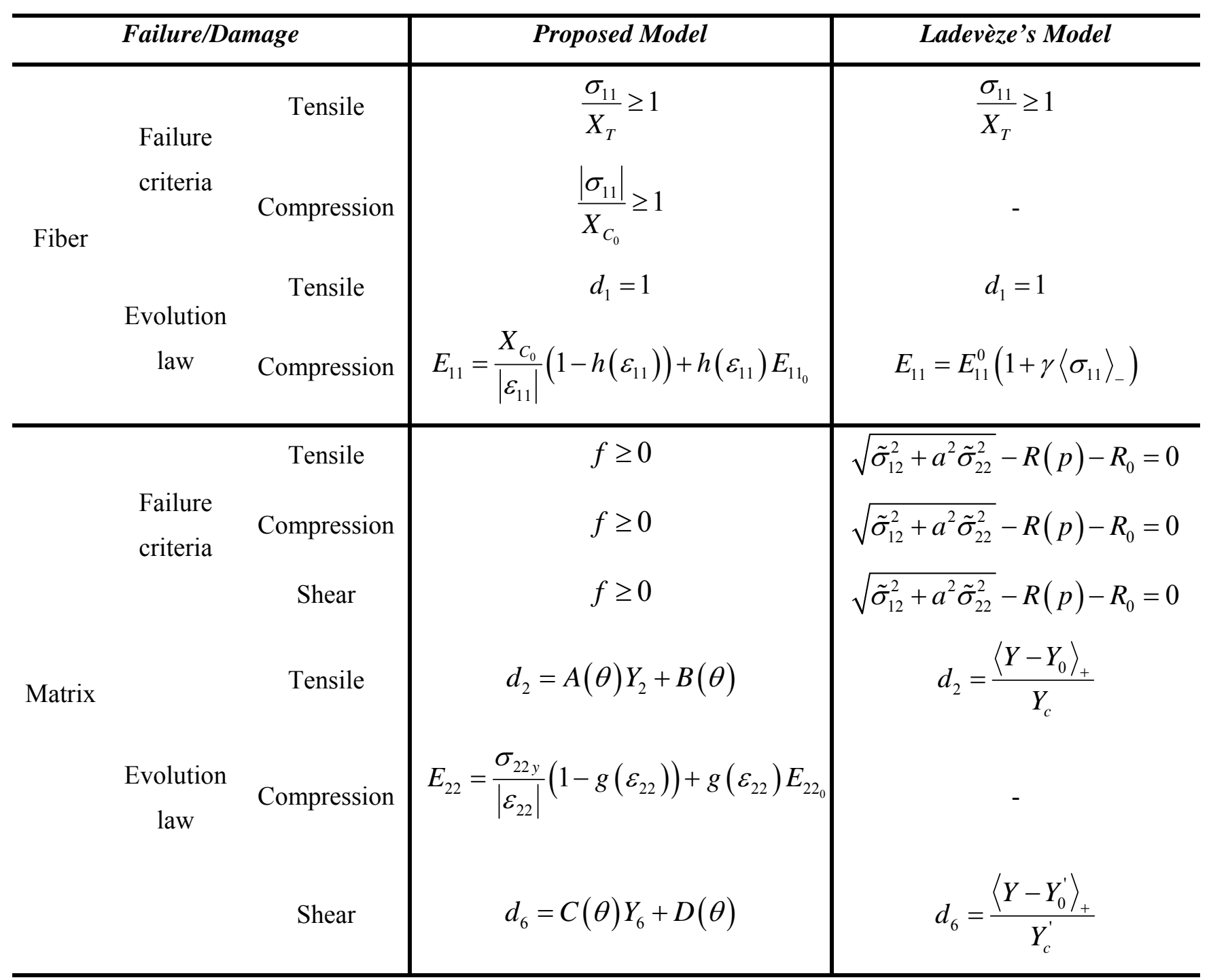

The differences start for the fiber compression failure criteria, where Ladevèze's model decreases the elastic parameter even at low loads level. However, for the model presented in this work, the elastic modulus decreases after, under compression, the load in fiber direction (direction 1) reaches some specified value, which is the limit between linear and nonlinear behavior (response).

Despite both material models use the secant modulus to decrease the elastic modulus $E_{11}$, the proposed model is the function of the strain and Ladevèze's model has a secant modulus $E_{11}=E_{11}^{0}\left(1+\gamma\left\langle\sigma_{11}\right\rangle_{-}\right)$, which decreases in a constant way.

The damage onset surface is also different for both material models. Ladevèze's model regards the matrix plasticity, using the parameters $R(p)$ and $R_{0}$. The proposed material model uses a curve fit of the experimental data to identify the damage onset. 
Finally, Ladevèze's model regards that the influence of the transverse and shear stress is constant. The material model presented in this work regards the damage variable as dependent on the ply orientation.

\subsection{PARAMETERS IdENTIFICATION AND MODEL EVAluATION EXPERIMENTS}

In order to identify the parameters and/or to evaluate the material model, standard tests for tension, shear and compression and some additional off-axis and angle-ply have been selected and performed.

Regarding Table 1, some special considerations must be taken for off-axis experiments. It is very difficult to apply only a uniaxial stress state in an off-axis test coupon due to test machine clamps. Although the applied load is uniaxial, the stress state is biaxial, with a shear stress component $\tau_{x y}$ present. A detailed discussion about off-axis experimental testing can be found in literature (Pierron \& Vautrin, 1996) and (Herakovich, 1998), which describes that the test coupon aspect ratio and lamina orientation have a significant influence on $\tau_{x y}$. Therefore, the present author followed the recommendations given by the literature. Also, Pierron \& Vautrin (1996) showed the effects of the end tabs in off-axis coupons tests.

As commented earlier, it is important to highlight that some tests have been used to evaluate the material model. For example, the off-axis $15^{\circ}$ and $30^{\circ}$ compression and, off-axis $30^{\circ}$ tensile test have been used for the model evaluation. In fact, those coupons have not been used for the elastic properties material characterization, once the coupon aspect ratio is too low. However, they are good test coupons to evaluate the model due to the complex stress state. More details about experiments as well as the results will be shown in the next chapters.

Another important aspect is related to the determination of the damage variables. For this case, cyclic experiments have been carried out and, the damage measurement procedure was the same as that described in the literature ( (Allix, Ladevèze, \& Vittecoq, 1994) (Ladeveze \& LeDantec, 1992)). For all experiments, the machine speed has been $0.5 \mathrm{~mm} / \mathrm{min}$ and, in order to avoid low cycle fatigue, the maximum number of cycles has been equal to 5 (five). The relation between damage variables and Thermodynamic Forces (Y) is shown in Figure 8 for $\mathrm{d}_{2}$ and, in Figure 9 for $\mathrm{d}_{6}$. 


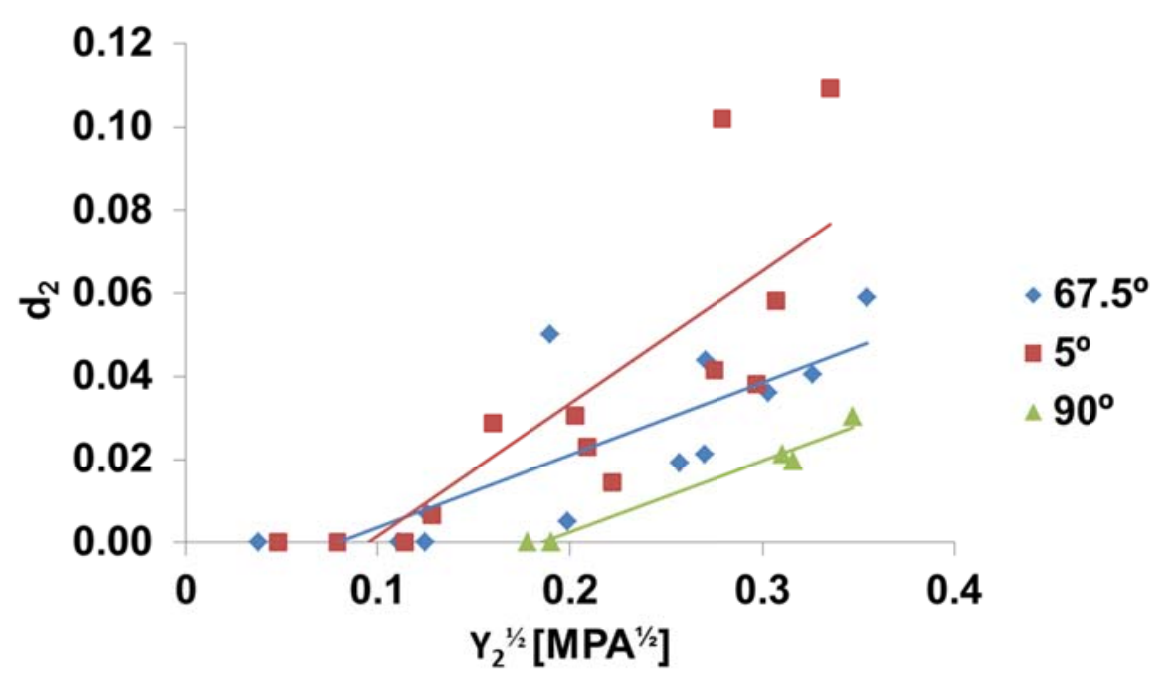

Figure 8: Damage evolution $-\mathrm{d}_{2}$ (see Figure 6 and EQ. (20) and EQ. (21)).

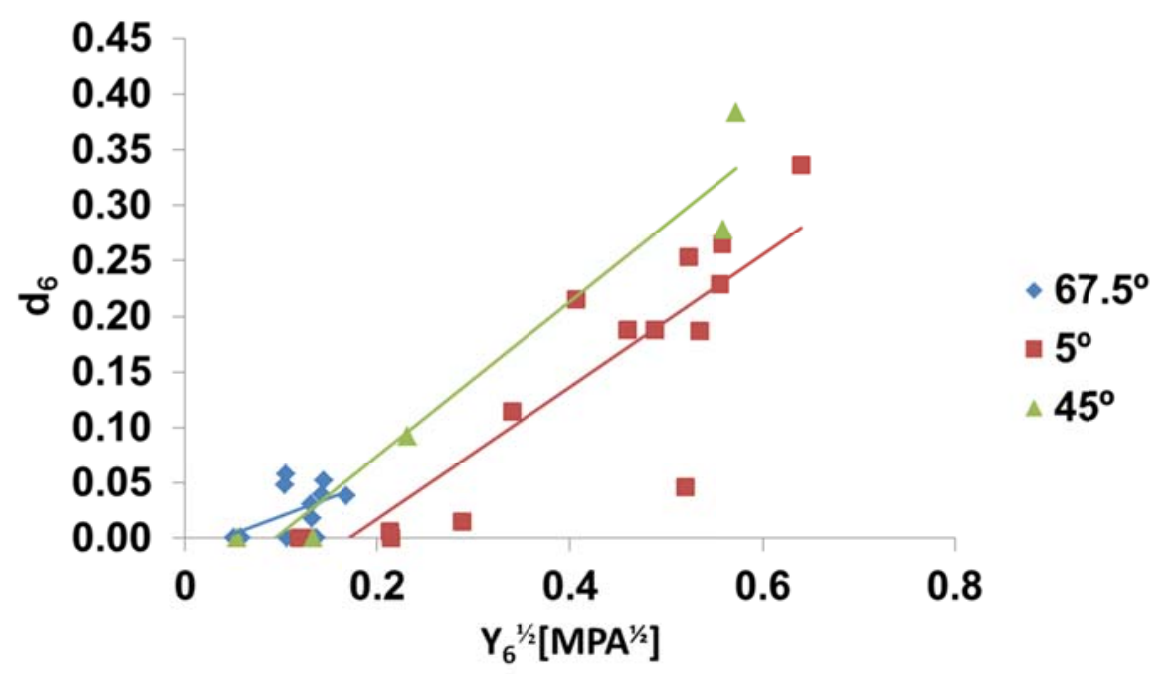

Figure 9: Damage evolution $-d_{6}$ (see Figure 6 and EQ. (20) and EQ. (21)).

For the experimental data shown in Figure 8 and Figure 9, the statistics for the linear regression are summarized in Table 3. 
Table 3: Data statistics for the regression

\begin{tabular}{l|ccc}
\hline Variable & R Square & Adjusted R Square & Significance of $F$ \\
\hline $\mathrm{d}_{2}-5^{\circ}$ & 0.51 & 0.48 & 0.0006 \\
$\mathrm{~d}_{2}-67.5^{\circ}$ & 0.63 & 0.60 & 0.0020 \\
$\mathrm{~d}_{2}-90^{\circ}$ & 0.47 & 0.43 & 0.0047 \\
$\mathrm{~d}_{6}-5^{\circ}$ & 0.76 & 0.74 & 0.00002 \\
$\mathrm{~d}_{6}-45^{\circ}$ & 0.95 & 0.93 & 0.005 \\
$\mathrm{~d}_{6}-67.5^{\circ}$ & 0.30 & 0.23 & 0.064 \\
\hline
\end{tabular}

Table 3 shows some important statistical parameters obtained by the linear regression. The $\mathrm{R}$ square represents how much of the output variables variance is explained by the input variables variance (ideally, this value should be greater than 0.6 ) by using this information, for $\mathrm{d}_{2}-5^{\circ}, \mathrm{d}_{2}-90^{\circ}$ and $\mathrm{d}_{6}-67.5^{\circ}$, the regression results are not so good, regarding the amount of data. The worst case is for $\mathrm{d}_{6}-67.5^{\circ}$ where only $30 \%$ of the predicted damage can be explained by the thermodynamic force. The other parameter, the adjusted $\mathrm{R}$ square is more conservative than the $\mathrm{R}$ square and, once again, the worst case is for $\mathrm{d}_{6}-67.5^{\circ}$.

On one hand, the R square and the adjusted R square are not good for some cases. On the other hand, the significance of $\mathrm{F}$ ( the regression might have been obtained by chance) is acceptable for almost all cases (values lower than $1 \%$ ), but for $\mathrm{d}_{6}-67.5^{\circ}$ the value of significance of $\mathrm{F}$ is $6.4 \%$, i.e. It is $6.4 \%$ possible that the regression had been a chance. Although some values identified are not so good, all results have been used in this work, even for $\mathrm{d}_{6}-67.5^{\mathrm{o}}$.

Regarding the evolution of the parameter $\mathrm{d}_{2}$ shown in Figure 8 , it is verified that the ply orientation has an important role in damage evolution. The damage process has been accelerated due to shear stress. For the parameter $\mathrm{d}_{6}$, as expected, if the orientation is close to $90^{\circ}$; the shear damage is low as shown by Figure 9 . However, if the ply orientation changes towards $0^{\circ}$; then $\mathrm{d}_{6}$ becomes a very important parameter and, it strongly affects the matrix damage process. As previously shown, the damage model assumes that $\mathrm{d}_{2}$ evolves in a linear way. Thus, $d_{2}$ evolution equations have been fitted as a function of the orientation angle $(\theta)$ (EQ. (20)). The same procedure has been applied to $d_{6}$ as presented by EQ. (20). Therefore, based on the experimental results, it is possible to identify the parameters $A(\theta), B(\theta), C(\theta)$ and $D(\theta)$. 


\subsection{IMPLEMENTATION VIA UMAT (USER MATERIAL SUbROUTINE)}

Figure 10 presents the flow chart of UMAT and ABAQUS ${ }^{\mathrm{TM}}$ integration for progressive failure analysis. ABAQUS ${ }^{\mathrm{TM}}$ controls the nonlinear solution process. The process consists of successive iterations. For all finite element integration points (at each lamina) and for every analysis time step, the UMAT subroutine is called mainly to calculate the stress state and the Jacobian matrix $(\partial \sigma / \partial \varepsilon)$ compatible to the strain state given for each $i$-th step. Thus, the first procedure performed by the UMAT is the identification of the material model state variables and the strain tensor. After that, the Jacobian matrix is calculated and, there is a prediction of the stress state (step 1 in Figure 10). Then, the calculated stress state is verified by the damage model implemented via UMAT (step 2 in Figure 10). If any failure mode occurs, the damage variables are updated (step 3 in Figure 10). After that, the state variables are updated with the damage variables, and then these variables are passed to the next step to update the stress tensor. If failure does not occur, the prediction of the stress state is considered correct. Then, a Residual $(\mathrm{R})$ is calculated as the difference between the internal and the external forces vectors, and it is compared to the tolerance threshold established by the user. If there is a convergence, i.e. $\mathrm{R}$ is lower than the tolerance (tolerance $=0.005$, which is adequate for engineering applications (Simulia, 2010)); then a new load step is applied. Otherwise, ABAQUS $^{\mathrm{TM}}$ stops the numerical analysis. During the iterations for each load step, it is expected that $\mathrm{R}$ decreases. If $\mathrm{R}$ increases, then the solution process diverges and, the numerical analysis stops. Divergence normally occurs when the material properties show a high degree of degradation and the structure has insufficient resistance to support the applied loads. Localisation problems can take place with the abrupt reduction of the material properties. The FE (Finite Element) mesh density plays an important role in this situation. Moreover, the material model hypotheses need to be physically consistent to avoid incoherent predictions. 

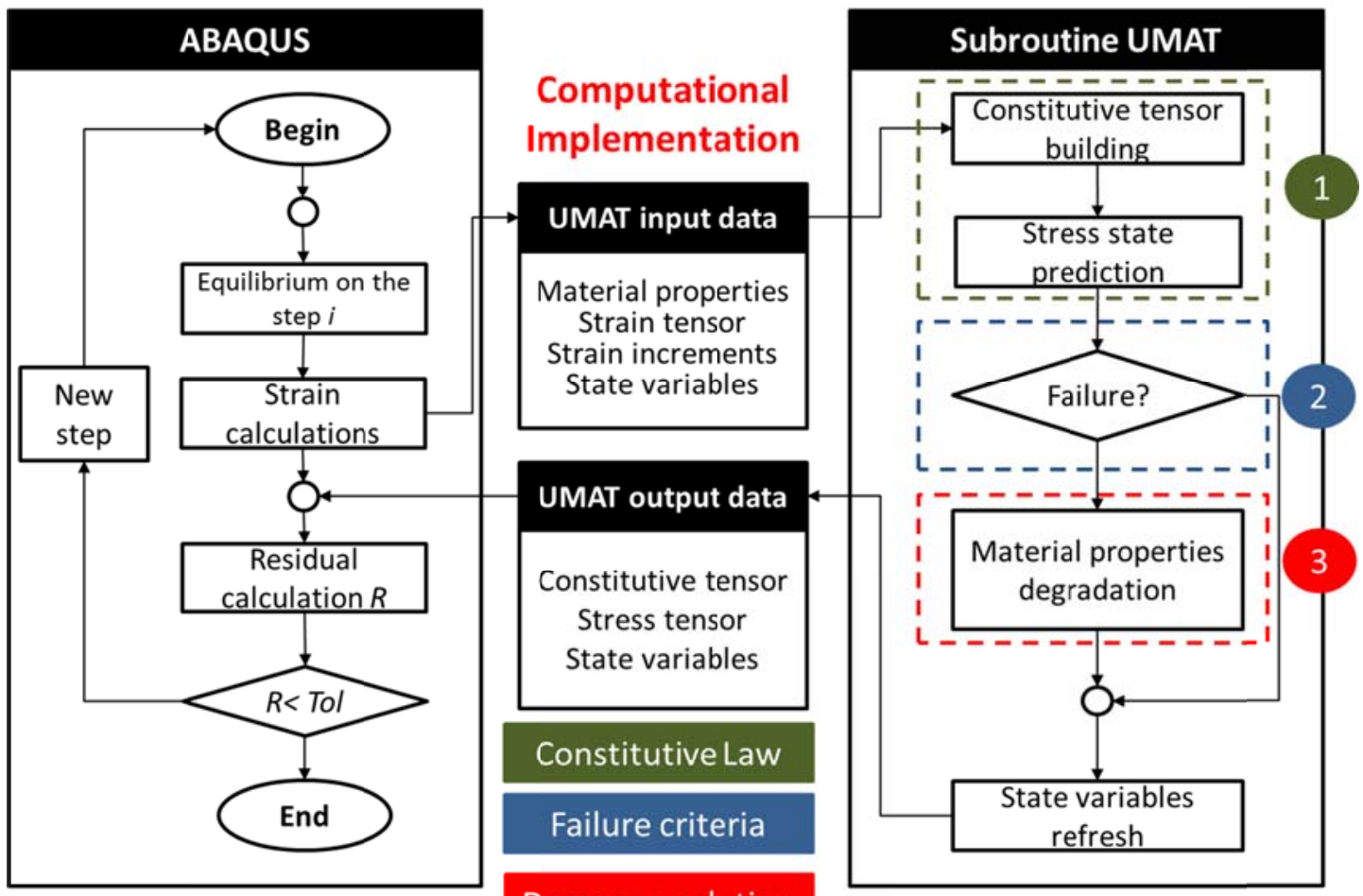

Damage evolution

Figure 10: Flow chart for ABAQUS ${ }^{\mathrm{TM}}$ and UMAT integration

\subsection{IMPACT SIMULATIONS}

Explicit dynamic simulations have some particularities, which make them different from quasi-static analyses. To clarify those issues, this section approaches explicit dynamic simulations characteristics.

A dynamic analysis must be performed when the model inertia is relevant to describe the event (see Figure 11). Modal analysis is also a dynamic simulation, but in this work, it will not deal with this kind of dynamic analysis.

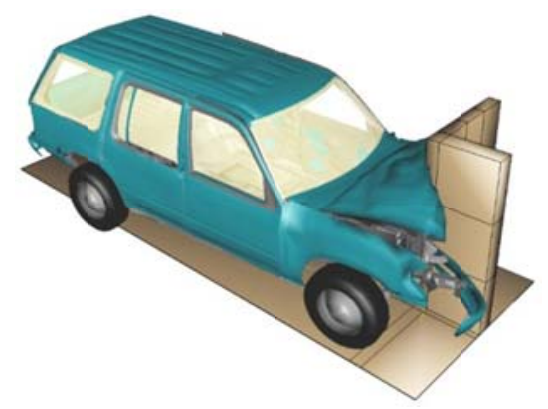

Figure 11: a vehicle impact simulation is an example of process to show that inertia effects are important. 
The equation of motion for dynamic simulation is given by:

$$
[M]\{\ddot{u}\}+[C]\{\dot{u}\}+[K]\{u\}=\{F\}
$$

Where $[\mathrm{M}]$ is the mass matrix, $[\mathrm{C}]$ is the damping matrix, $[\mathrm{K}]$ is the stiffness matrix, $\{\mathrm{F}\}$ is the load vector and $\{u\}$ is the displacement vector. From EQ. (27), the material density has always been used to calculate the mass matrix, but the damping values can be neglected, if damping effects are not relevant.

\subsubsection{MASS MATRIX}

The mass matrix is a generalization of the mass concept to the generalized coordinates used in the FEM. The finite element mass matrix is based on the element mass matrix in local coordinates transformed into global coordinates. And all the element mass matrices are merged, forming the model mass matrix, like the technique used for the stiffness matrix.

The consistent mass matrix is defined by:

$$
M=\int_{\Omega} \rho \psi^{T} \psi d \Omega
$$

Where $\rho$ is the density, $\psi$ is the shape function (using Galerkin, the trial and weight functions are the same).

In opposite to the stiffness matrix, the mass matrix can be handled, for example, by making a diagonal mass matrix to improve the numerical solution of the differential equations. There are different methods to modify the mass matrix. Each approach to obtain the mass matrix affects the performance of the solution via FEM (Zienkiewicz \& Taylor, 2000).

As mentioned before, to improve the numerical solution for many applications, the consistent mass matrix can be modified to become a diagonal matrix. The first procedure to build a diagonal mass matrix consists of summing all the matrix line components ( $\left.M_{i i}=\sum_{j} M_{i j}\right)$. And the procedure consists of calculating $M_{i i}^{*}=\frac{S}{D} M_{i i}$, where $\mathrm{S}$ is the 
summation of the consistent mass matrix and D is the summation of the consistent mass matrix principal diagonal.

\subsubsection{DAMPING MATRIX}

Real structures exhibit some levels of energy dissipation due to material nonlinearities, internal or external friction, damage, etc. Steel and high strength aluminum possess low internal damping. On the other hand, some composite materials can possess high damping.

Damping can be modeled in several ways in ABAQUS ${ }^{\mathrm{TM}}$ in order to accurately simulate the energy loss. There are four categories of damping source in ABAQUS ${ }^{\mathrm{TM}}$ (Dassault Systèmes Simulia Corp, 2010):

Material and element damping: specified as material properties and specific elements such as dashpots, springs and connectors, which could work as dampers (viscous and structural);

Global damping: apply damping to the entire model for the cases which are not possible to use material and element damping.

Modal damping: apply damping to the system modes.

Damping associated with time integration: "Marching through a simulation with finite time increment size cause some damping." (Simulia, 2010).

Also, damping can be simulated as a viscous model, which is proportional to either velocity or displacement.

ABAQUS $^{\mathrm{TM}}$ provides the Rayleigh's model for direct integration dynamic analysis to simulate energy dissipation mechanisms through damping (Dassault Systèmes Simulia Corp, 2010). In fact, in the Finite Element Analysis (FEA), damping is treated as a matrix, which can be approached in two different ways: either as a material property or as a numerical object to oppose the excitation forces (Kyriazoglou \& Guild, 2007).

Considering the equilibrium equation in dynamic analysis:

$$
[M] \cdot\{\ddot{u}\}+[C] \cdot\{\dot{u}\}+[K] \cdot\{u\}=f(t)
$$


According to (Zabaras \& Pervez, 1990), the following transformation can be applied to EQ. (29), $u_{i}=M_{i j}^{-1 / 2} y_{j}$, and the resulting equation is multiplied by $M_{i j}^{-1 / 2}$. Thus, EQ.(27) can be rewritten as:

$$
\{\ddot{y}\}+[\tilde{C}]\{\dot{y}\}+[\tilde{K}]\{y\}=\{\tilde{f}\}
$$

EQ.(30) is used to obtain the critical damping, which is calculated by:

$$
C_{C R_{i j}}=2 \tilde{K}_{i j}^{1 / 2}
$$

Based on the classical modal analysis $([C]=0,\{f\}=0)$, it can be shown that $[\phi]^{t}[K][\phi]=[w]^{2}$. The modal matrix of eigenvalues vector is $\tilde{\phi}_{i j}=M_{k j}^{1 / 2} \phi_{i l}$. Thus, the modal fraction of critical damping is given by:

$$
\xi_{i}=\frac{\{\phi\}_{i}^{t}[C]\{\phi\}_{i}}{\{\tilde{\phi}\}_{i}^{t}\left[C_{C R}\right]\{\tilde{\phi}\}_{i}}
$$

Using the Principle of the Orthogonality for $\{\tilde{\phi}\}$, eq(32) results in:

$$
\{\phi\}_{i}^{t}[C]\{\phi\}_{i}=2 \xi_{i} \omega_{i}
$$

Rayleigh's model introduces damping in the structure as a linear combination of mass and stiffness system matrices (Kyriazoglou \& Guild, 2007), where the parameters $\alpha$ and $\beta$ can be obtained by using both EQ. (34) and experimental data.

$$
[C]=\alpha[M]+\beta[K]
$$


Therefore, the damping is proportional to the mass and the stiffness. The mass contribution is related to the low frequencies vibrations and the stiffness contribution is related to the high frequencies vibrations (Dassault Systèmes Simulia Corp, 2010).

It can be proved that for a mode $i$, the fraction of critical damping proportional to $[\mathrm{M}]$ is:

$$
\xi_{i}=\frac{\alpha}{2 \omega_{i}}
$$

And, the fraction of critical damping proportional to $[\mathrm{K}]$ is:

$$
\xi_{i}=\frac{\beta \omega_{i}}{2}
$$

In fact, for this work, a reversal analysis was performed in order to obtain Rayleigh's parameters. However, in the coming works, these parameters may be obtained by dynamic experimental analyses.

\subsubsection{NUMERICAL SOLUTION: IMPLICIT VS. EXPLICIT}

To perform a dynamic analysis, it is possible to apply both implicit and explicit methods. Each method possesses its own advantages and disadvantages and finite element software user must be aware of each method particularities.

Implicit methods are unconditionally stable. Despite the stability, these methods may face some problems when analyzing the complicated 3D models. The reasons for these problems are related to the decrease of time increment; so computational cost for calculation of tangent stiffness matrix will increase radically and it may diverge. Also, local instabilities could lead to difficulties in order to guarantee the equilibrium (Sun, Lee et al., 2000).

Explicit methods have been used to overcome the problems with implicit method mentioned in previous paragraph. For explicit methods, the computational cost is proportional to the size of the finite element model and does not increase radically like solution using implicit methods. Although the explicit methods may overcome the implicit problems, they are conditionally stable. The stability of explicit method is limited to the size of the time increment, which should be less than the dilatational wave in the finite element. In addition, these methods are limited for short transient problems (Sun, Lee et al., 2000). 


$$
\Delta t \leq L^{e l} \sqrt{\frac{\rho}{\lambda+2 \mu}}
$$

Where $\rho$ is the density, $\lambda$ and $\mu$ are the material properties (Lamé constants).

Small time increments allow solving the problems without calculating the tangent stiffness matrix, what is very interesting for contact problems.

Despite the problems previously discussed, if the finite element model contains very small or stiff elements, the efficiency of explicit integration decreases once the time increment of the entire mesh will be set by those elements. In these cases, some special techniques are necessary to perform the explicit simulations. One of these techniques is called mass scaling, where the mass of those elements is increased. The other technique is called subcycling, where a smaller time step is used for those elements (Belytschko, Liu et al., 2000). Mass scaling has been used when high frequencies are not important as for quasi-static simulations. Regarding subcycling method, the domain is divided into subdomains and, each subdomain is integrated with its own stable time step (Belytschko, Liu et al., 2000).

Explicit methods could be used to perform quasi-static simulations (e.g. simulation of tensile test) when the inertia effects are neglected but the ratio between kinetic energy and internal energy is less than 10\% (ABAQUS 6.10 User Manual, 2010).

$$
\frac{E_{k}}{E_{i}} \leq 10 \%
$$

To solve dynamic problems the finite difference method allows writing the speed and acceleration as:

$$
\begin{aligned}
& \{\dot{u}\}_{i+\alpha}=\frac{\{u\}_{i+1}-\{u\}_{i}}{\Delta t} \\
& \{\ddot{u}\}_{i+\beta}=\frac{\{\dot{u}\}_{i+1}-\{\dot{u}\}_{i}}{\Delta t}
\end{aligned}
$$


With $0 \leq \alpha, \beta \leq 1$. These constants come from an integration algorithm, assuming the hypothesis that the speed and acceleration are a linear combination of the interval limits speed and acceleration. In other words:

$$
\begin{aligned}
& \{\dot{u}\}_{i+\alpha}=\alpha\{\dot{u}\}_{i+1}+(1-\alpha)\{\dot{u}\}_{i} \\
& \{\ddot{u}\}_{i+\alpha}=\beta\{\ddot{u}\}_{i+1}+(1-\beta)\{\ddot{u}\}_{i}
\end{aligned}
$$

Assuming $\alpha=\beta=1 / 2$, the speed and acceleration are the average values of the interval. Using $\alpha=1 / 2$ in (38) and (39) yields:

$$
\begin{gathered}
\{\dot{u}\}_{i+1}=\{\dot{u}\}_{i}+(1-\beta)\{\ddot{u}\}_{i} \Delta t+\beta\{\ddot{u}\}_{i+1} \Delta t \\
\{u\}_{i+1}=\{u\}_{i}+\{\dot{u}\}_{i} \Delta t+\frac{1}{2}(1-\gamma)\{\ddot{u}\}_{i} \Delta t^{2}+\frac{1}{2} \gamma\{\ddot{u}\}_{i+1} \Delta t^{2}
\end{gathered}
$$

Where $\gamma(0 \leq \gamma \leq 1)$ is another constant. Applying (42), (43) and (44) to the motion equation despite damping effects the dynamic problems can be defined as:

$$
[M]\{\ddot{u}\}+[K]\{u\}=\{F\}
$$

To solve the dynamic problem, it is possible to apply either the explicit or implicit methods. Table 4 presents a simple schema of those methods. 
Table 4: Explicit vs. Implicit integration algorithm.

\begin{tabular}{|c|c|}
\hline Explicit & Implicit \\
\hline $\begin{array}{c}\gamma=0 \text { in (44) } \\
\{u\}_{i+1}=\{u\}_{i}+\{\dot{u}\}_{i} \Delta t+\frac{1}{2}\{\ddot{u}\}_{i} \Delta t^{2} \\
\{\ddot{u}\}=[M]^{-1}[\{F\}-[K]\{u\}] \\
\{\dot{u}\}_{i+1}=\{\dot{u}\}_{i}+(1-\beta)\{\ddot{u}\}_{i} \Delta t+\beta\{\ddot{u}\}_{i+1} \Delta t\end{array}$ & 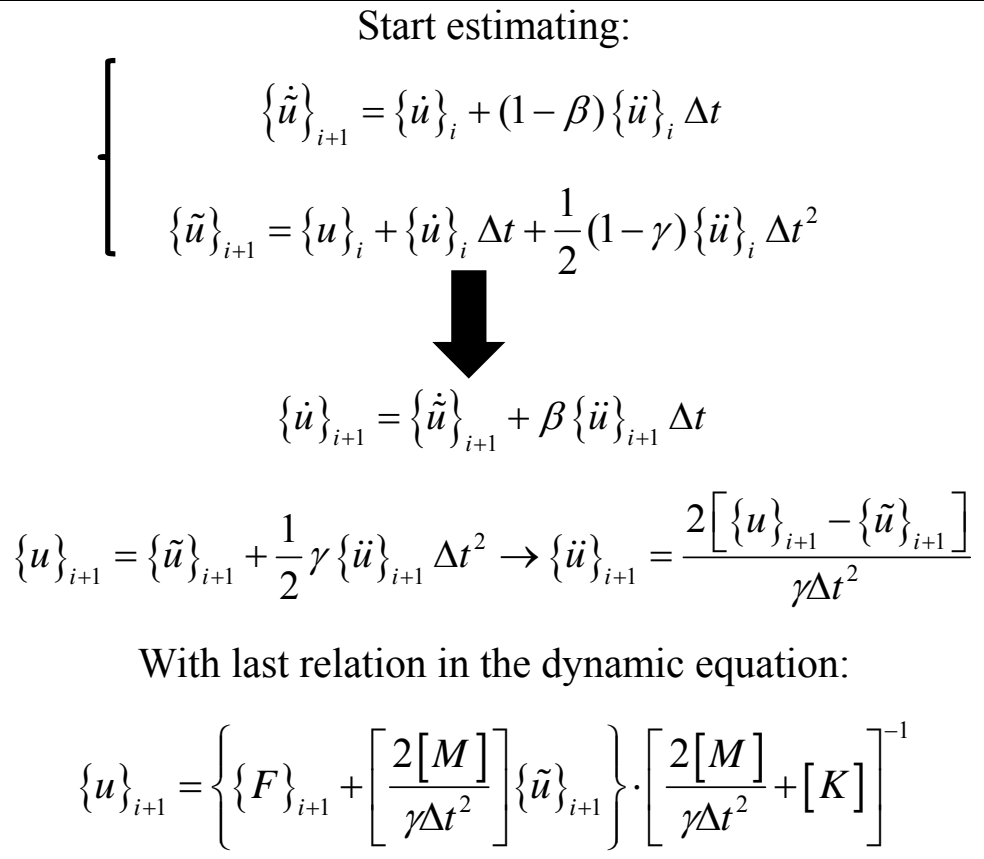 \\
\hline
\end{tabular}

It is important to verify that an implicit algorithm requires the calculation of the stiffness matrix inversion. However, it is not necessary for an explicit algorithm. Also for an implicit method, with $\{u\}_{i+1}$, it is necessary to correct the initial value for the displacement and speed vectors. Thus, an iterative method, e.g. Newton-Raphson, is necessary to be used.

In the next section, the explicit algorithm used in ABAQUS ${ }^{\mathrm{TM}} /$ Explicit is explained in further details.

\subsubsection{EXPLICIT SIMULATIONS VIA ABAQUS ${ }^{\text {TM }}$}

As mentioned before, the explicit method has been used to overcome some problems produced by the implicit method.

ABAQUS $^{\mathrm{TM}} /$ Explicit is established by using the explicit integration rule with lumped mass-matrix. The equation of motion is integrated by using the central difference method. This method is developed from central difference formulas by $\dot{u}$ and $\ddot{u}$ :

$$
\dot{u}^{i+1 / 2}=\frac{1}{\Delta t^{i+1 / 2}}\left(u^{i+1}-u^{i}\right)
$$


Where:

$$
u^{i+1}=u^{i}+\Delta t^{i+1 / 2} \dot{u}^{i+1 / 2}
$$

The acceleration is:

$$
\ddot{u}^{i}=\left(\frac{\dot{u}^{i+1 / 2}-\dot{u}^{i-1 / 2}}{t^{i+1 / 2}-t^{i-1 / 2}}\right)=\left(\frac{\Delta t^{i-1 / 2}\left(u^{i+1}-u^{i}\right)-\Delta t^{i+1 / 2}\left(u^{i}-u^{i-1}\right)}{\Delta t^{i+1 / 2} \Delta t^{i-1 / 2} \Delta t^{i}}\right)
$$

For equal time steps, $\ddot{u}$ becomes:

$$
\ddot{u}^{i}=\left(\frac{u^{i+1}-2 u^{i}+u^{i-1}}{\left(\Delta t^{i}\right)^{2}}\right)
$$

The nodal velocities and displacements update can be obtained without solving any equations, once the mass matrix is diagonal. "In explicit method, the time integration of the discrete momentum equations does not require the solution of any equations" (Belytschko, Liu et al., 2000).

The equation of motion is:

$$
\ddot{u}^{i}=M^{-1}\left(F^{i}-I^{i}\right)
$$

The time increment must satisfy $\Delta t \leq \frac{2}{w_{\max }}$, where $w_{\max }$ the maximum element eigenvalue is. In Belytschko, Liu \& Moran (2000), it is possible to observe further details about algorithm for explicit simulation as described below:

1. Set initial conditions and parameters $\left(\dot{u}^{0}, \sigma^{0} ; u^{0}=0, i=0, t=0\right)$ and compute $M$;

2. Get force;

3. Compute the initial accelerations: $\ddot{u}^{i}=M^{-1}\left(f^{i}-C^{\text {damp }} \dot{u}^{i-1 / 2}\right)$;

4. Update time: $t^{i+1}=t^{i}+\Delta t^{i+1 / 2}, t^{i+1 / 2}=\frac{1}{2}\left(t^{i}+t^{i+1}\right)$;

5. Update first partial nodal velocities : $\dot{u}^{i+1 / 2}=\dot{u}^{i}+\left(t^{i+1 / 2}-t^{i}\right) \ddot{u}^{i+1 / 2}$; 
6. Enforce the velocity boundary conditions: if node $I$ on $\Gamma_{v_{i}}: \dot{u}_{i I}^{i+1 / 2}=\overline{\dot{u}}$

7. Update nodal displacements: $u^{i+1}=u^{i}+\Delta t^{i+1 / 2} \dot{u}^{i+1 / 2}$;

8. Get force;

9. Compute $a^{i+1}$;

10. Update second partial nodal velocities $\dot{u}^{i+1}=\dot{u}^{i+1 / 2}+\left(t^{i+1}-t^{i+1 / 2}\right) \ddot{u}^{i+1}$;

11. Check the energy balance for step $i+1$;

12. Update $i \leftarrow i+1$

13. Output; if simulation is not done back to step 4

Subroutine Get force:

1. Initialize $f^{i}=0, \Delta t_{c r i t}=\infty$;

2. Compute global external forces $f_{\text {ext }}^{i}$;

3. Loop over elements:

a. Gather both element nodal displacements and velocities;

b. $f_{e}^{\text {int, } i}=0$;

c. Loop over quadrature points $\xi_{Q}$

i. If $i=0$, go to iv;

ii. Compute measures of deformation: $D^{i-1 / 2}\left(\xi_{Q}\right), F^{i}\left(\xi_{Q}\right), E^{i}\left(\xi_{Q}\right)$;

iii. Compute the stress $\sigma^{i}\left(\xi_{Q}\right)$ using the constitutive equation;

iv. $f_{e}^{\mathrm{int}, i} \leftarrow f_{e}^{\mathrm{int}, i}+\left.\Delta f\right|_{\xi_{Q}} ;$

d. Compute external nodal forces on element: $f_{e}^{e x t, i}$;

e. $f_{e}^{i}=f_{e}^{e x t, i}-f_{e}^{\mathrm{int}, i}$;

f. Compute $\Delta t_{\text {crit }}^{e}$, if $\Delta t_{c r i t}^{e}<\Delta t_{\text {crit }}$ then $\Delta t_{\text {crit }}=\Delta t_{\text {crit }}^{e}$;

g. Scatter $f_{e}^{i}$ to global $f^{i}$;

4. End Loop over elements;

5. $\Delta t=\alpha \Delta t_{c r i t}$

In fact, numerous methods to solve problems, where the inertia is relevant, are available at ABAQUS $^{\mathrm{TM}}$. However, in summary, ABAQUS/Standard has used implicit operators for integration of the equations of motion and ABAQUS/Explicit has used the central difference method as commented earlier. In implicit dynamic analysis, as required to invert the integration operator matrix (see the algorithm for implicit dynamics - Table 4), a set of nonlinear equilibrium equations must be solved for every time increment. On the other hand, for explicit dynamic analysis (see the explicit dynamic algorithm - Table 4), the displacements 
and velocities are known in the beginning of the time increment. Therefore, the global mass matrix and stiffness matrix have not been inverted.

In addition, regarding the element types, ABAQUS/Explicit offers fewer elements than ABAQUS/Standard and when a nonlinear analysis is carried out, the user must consider the length of the time step, once the response is compared to the stability limit.

Finally, just out of curiosity, ABAQUS ${ }^{\mathrm{TM}}$ allows using of nonphysical material properties, e.g. negative mass value, negative damping and negative stiffness. Sometimes, these material nonphysical properties are used to adjust the FE model.

\subsection{Conclusions}

This chapter described the development of a new material model for unidirectional long fiber composite laminates based on CDM.

The differences between the proposed material model and other classical material models are:

$\checkmark$ Degradation of elastic properties for fiber under compression is more accurate in the proposed model, once the equation used fits better the experimental data;

$\checkmark$ The damage onset surface is based on experimental data;

$\checkmark$ As for fiber direction (direction 1) under compression, the elastic properties degradation for compression of transverse direction is also more accurate in this model;

The damage parameters $\mathrm{d}_{2}$ and $\mathrm{d}_{6}$ calculation are dependent on the ply orientation accounting the influence of shear stress and transverse stress on the damage evolution.

To verify the material model performance, firstly the model will be applied to simulate flat carbon fiber composite coupons under simple loading conditions, and then, it will be checked for more complex loading conditions as the four point bending. At last, the model performance will be evaluated to simulate the impact on carbon fiber filament winding cylinders. 
A set of experiments in this work consists of characterizing the material elastic properties and determining the strength values for the carbon fibers composite material used in this research. Another set of experiments is used to identify the material model parameters as the damage variables $\mathrm{d}_{2}$ and $\mathrm{d}_{6}$, and to evaluate the potentialities of the model as well.

Therefore, the experiments can be subdivided into two main groups: characterization/identification and evaluation. Regarding the evaluation experiments, they are subdivided into quasi-static and dynamic experiments (impact tests).

\subsection{Quasi-Static Tests}

Table 5 shows the quasi-static tests performed in this work. This table shows the type of test (compression or tensile), the coupon lay-up, the ASTM standard used as well as the material parameters obtained by each type of test.

Table 5: Quasi Static Experiments

\begin{tabular}{ccccc}
\hline Test & Standard & $\begin{array}{c}\text { Dimensions } \\
{[\mathbf{m m}]}\end{array}$ & $\begin{array}{c}\text { Number of } \\
\text { specimens }\end{array}$ & $\begin{array}{c}\text { Material } \\
\text { Parameters }\end{array}$ \\
\hline Tensile $0^{\circ}$ & ASTM D3039 & $250 \times 15 \times 1$ & 5 & $E_{11}, v_{12}, X_{T}$ \\
\hline Tensile $90^{\circ}$ & ASTM D3039 & $175 \times 25 \times 2$ & 5 & $E_{22}, d_{2}, Y_{T}$ \\
\hline Tensile $\pm 67.5^{\circ}$ & N.A. & $175 \times 25 \times 2$ & 5 & $d_{2}$ and $d_{6}$ \\
\hline Tensile $5^{\circ}$ & N.A. & $175 \times 25 \times 2$ & 5 & $d_{2}$ and $d_{6}$ \\
\hline In-plane Shear $\pm 45^{\circ}$ & ASTM D3518 & $250 \times 25 \times 2.7$ & 5 & $G_{12}, S_{12}, d_{6}, S_{12_{y}}$ \\
\hline Compression $0^{\circ}$ & ASTM D3410 & $150 \times 10 \times 2$ & 7 & $X_{C_{0}}, X_{C}, \sigma_{11_{0}}$ \\
\hline Compression $90^{\circ}$ & ASTM D3410 & $150 \times 25 \times 2$ & 7 & $Y_{C}, \sigma_{22_{0}}, E_{D_{C}}$ \\
\hline Compression $30^{\circ}$ & N.A. & $150 \times 25 \times 2$ & 7 & $d_{2}$ and $d_{6}$ \\
\hline Compression $15^{\circ}$ & N.A. & $150 \times 25 \times 2$ & 7 & $d_{2}$ and $d_{6}$ \\
\hline
\end{tabular}

The reason for choosing the off-axis and angle-ply coupons is to study the coupling effects between $\tau_{12}$ and $\sigma_{22}$ and how these stresses affect the damage evolution. Figure 12 
shows the failure modes vs. orientation of the fibers. Hence, it is possible to observe how the failure mode is affected by the fibers orientation in the ply.

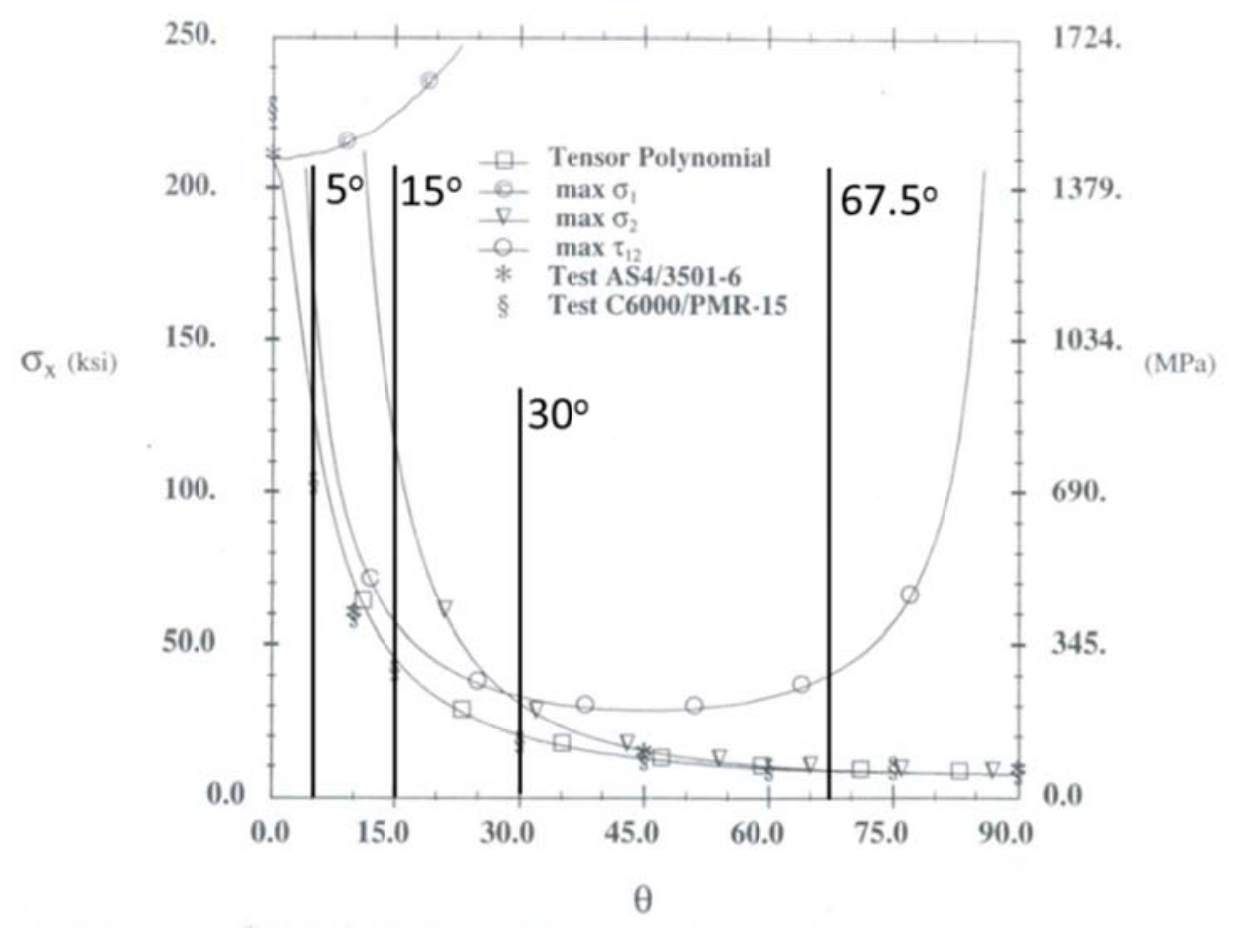

Figure 12: Failure modes vs. fibers orientation (Herakovich, 1998).

In the following sections, each of experiments will be explained in details and the respective results will be discussed.

\subsubsection{Tensile Tests}

The tensile tests follow the ASTM D3039 (2006) recommendations for $\left[0^{\circ}\right]_{10},\left[90^{\circ}\right]_{10}$, angle-ply $\left[ \pm 67.5^{\circ}\right]_{10}$ and off-axis $\left[5^{\circ}\right]_{10}$ filament winding carbon fiber coupons.

The $0^{\circ}$ coupons had $15 \mathrm{~mm}$ of width, $250 \mathrm{~mm}$ of length and $1.0 \mathrm{~mm}$ of total thickness $(0.1$ $\mathrm{mm}$ per layer). The $90^{\circ}$ coupons had $25 \mathrm{~mm}$ of width, $175 \mathrm{~mm}$ of length and $2.0 \mathrm{~mm}$ of total thickness $\left(0.2 \mathrm{~mm}\right.$ per layer) (ASTM D3039, 2006). The non-standard coupons $\left(\left[ \pm 67.5^{\circ}\right]_{10}\right.$ and $\left[5^{\circ}\right]_{10}$ ) had the same dimensions used for the $90^{\circ}$ ones. 


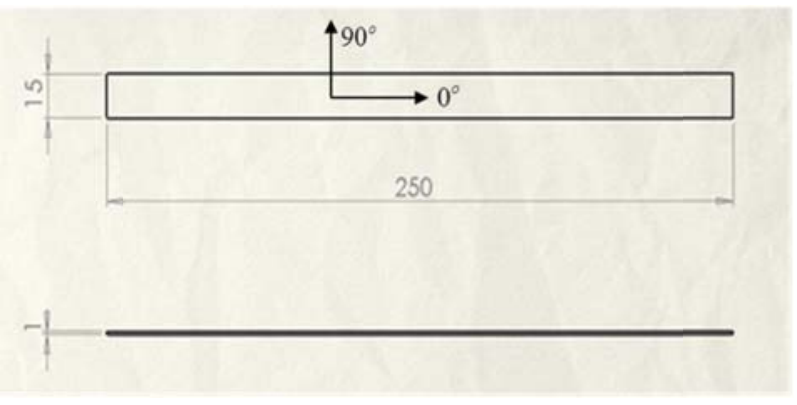

(a)

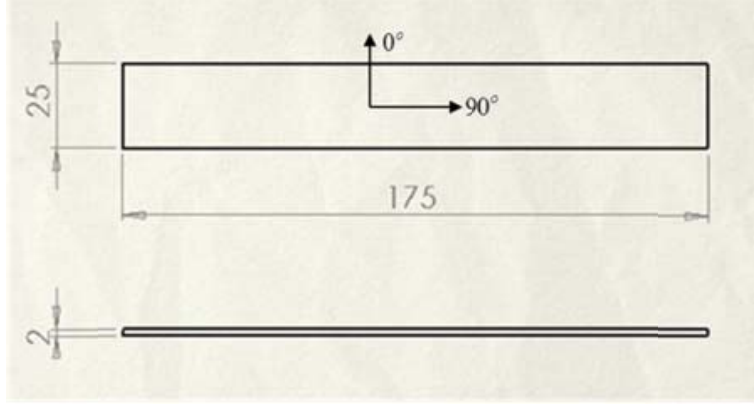

(b)

Figure 13: (a) $0^{\circ}$ coupon dimensions; (b) $90^{\circ}$ coupons dimensions (Unit: $\mathrm{mm}$ ).

The test machine provided force and displacement data where the test speed was equal to $0.5 \mathrm{~mm} / \mathrm{min}$ for loading and unloading cycles (Figure 14). Before cyclic tests, monotonic tests had been performed (for $0^{\circ}, 90^{\circ}, \pm 67.5^{\circ}$ and $5^{\circ}$ coupons) to know the general behavior of the material, determining the force vs. displacement curve. This information has been used for planning the maximum and minimum limits for each loading-unloading cycle. A limit of 5 (five) cycles has been used in order to avoid low cycle fatigue (Allix, Ladevèze, \& Vittecoq, 1994). To investigate the damage evolution, each loading-unloading cycle has been planned to begin the unloading at different load levels for each cycle. All cycles had the same low load level. Table 6 shows the purpose of each test and where each test has been carried out.

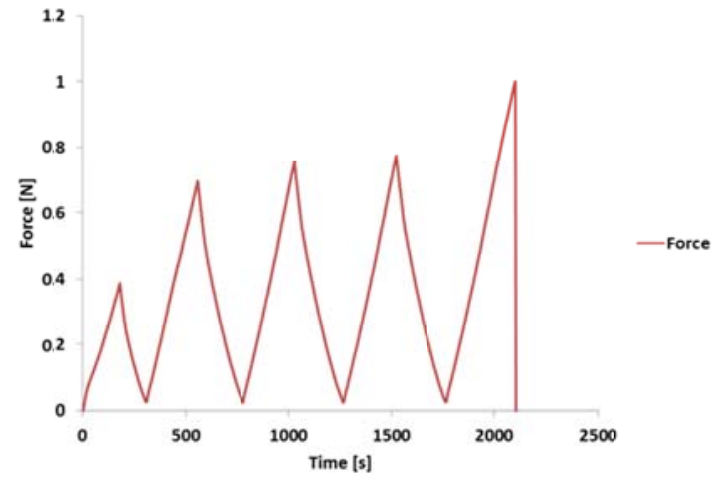

(a)

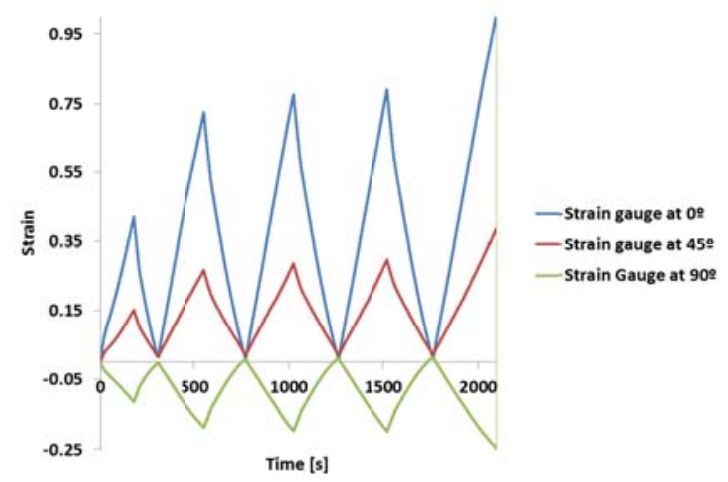

(b)

Figure 14: (a) Force vs. time; (b) Strain vs. time for a rosette strain gage 
Table 6: Identification and validation experiments

\begin{tabular}{ccc}
\hline Test & Laboratory & Type \\
\hline Tensile $0^{\circ}$ & USP $^{(*)}$ and KUL $\left.{ }^{(* *}\right)$ & Identification and Validation \\
\hline Tensile $90^{\circ}$ & $\mathrm{USP}^{(*)}$ and KUL ${ }^{(*)}$ & Identification and Validation \\
\hline Tensile $\pm 67.5^{\circ}$ & $\mathrm{KUL}^{(* *)}$ & Identification \\
\hline Tensile $5^{\circ}$ & $\mathrm{KUL}^{(* *)}$ & Identification \\
\hline In-plane Shear $\pm 45^{\circ}$ & $\mathrm{USP}^{(*)}$ & Identification \\
\hline Compression $0^{\circ}$ & $\mathrm{KUL}^{(* *)}$ & Identification and Validation \\
\hline Compression $90^{\circ}$ & $\mathrm{KUL}^{(* *)}$ & Identification and Validation \\
\hline 4-Point Bending $30^{\circ}$ & $\mathrm{KUL}^{(* *)}$ & Validation \\
\hline${ }^{(*)}$ USP: Material Engineering Department at University of Sao Paulo (Brazil) \\
${ }^{(* *)}$ KUL: Materials Engineering Department at KU Leuven (Belgium)
\end{tabular}

\subsubsection{Materials and Methods}

All tensile, compression, bending and shear tests are considered as quasi-static experiments. Those tests have been performed by using conventional, off-axis and angle-ply coupons. All coupons have been manufactured by using a filament winding process. Having two larger faces, the parallelepiped shape mandrel allows the plate wounding. Almost flat laminate plates made of carbon fiber with epoxy resin have been manufactured. After that, the plates are cured in a controlled oven. Then, in the final of this process, it is possible to obtain almost flat plates with a single curvature, which radius is around $7657 \mathrm{~mm}$. Those almost flat plates have been cut into coupons, following the dimensions provided by the American Society for Testing and Materials (ASTM) standards ( (ASTM D3039, 2006), (ASTM D790, 2008)) as commented earlier. It is important to highlight that the manufacturing processes described above have been carried out by the Brazilian Navy Technology Center in São Paulo (CTM-SP). Due to an agreement between Brazilian Navy Technology Center in São Paulo (CTM-SP) and Aeronautic Structural Group of Engineering School of São Carlos (Aeronautical Engineering Department - University of Sao Paulo - Brazil), all information about the manufacturing processes and material is classified. Therefore, the elastic properties and strength values cannot be shown in this work. In order to aid the readers for understanding the mechanical behavior of composite material obtained by CTM-SP, it is possible to mention that the CTM's material is similar (elastic and strength values) to the composite material investigated by Tita (2003) during his Ph.D. Thesis. The specimens manufactured and studied by Tita (2003) were made of prepreg M10 from Hexcel $^{\mathrm{TM}}$. In fact, they were unidirectional carbon fibers with epoxy resin and the fiber volume ratio was equal 
to $63 \%$. The elastic properties and strength values are shown either in Table 7 or by Tita, Carvalho, \& Vandepitte (2008).

Table 7: Elastic properties and strength values (Tita V. , 2003)

\begin{tabular}{cc}
\hline \multicolumn{3}{c}{ Elastic Properties } \\
\hline $\mathrm{E}_{11}$ & $127 \mathrm{GPa}$ \\
\hline $\mathrm{E}_{22}$ & $10 \mathrm{GPa}$ \\
\hline $\mathrm{G}_{12}=\mathrm{G}_{13}$ & $544 \mathrm{GPa}$ \\
\hline $\mathrm{G}_{23}$ & $3.05 \mathrm{GPa}$ \\
\hline$v_{12}=v_{13}$ & 0.34 \\
\hline $\mathrm{v}_{23}$ & 0.306 \\
\hline Density & $1580 \mathrm{~kg} / \mathrm{m}^{3}$ \\
\hline $\mathrm{X}_{\mathrm{T}}$ & $1400 \mathrm{MPa}$ \\
\hline $\mathrm{X}_{\mathrm{C}}$ & $930 \mathrm{MPa}$ \\
\hline $\mathrm{Y}_{\mathrm{T}}$ & $47 \mathrm{MPa}$ \\
\hline $\mathrm{Y}_{\mathrm{C}}$ & $130 \mathrm{MPa}$ \\
\hline $\mathrm{S}_{12}=\mathrm{S}_{13}$ & $53 \mathrm{MPa}$ \\
\hline $\mathrm{S}_{23}$ & $89 \mathrm{MPa}$ \\
\hline
\end{tabular}

After obtaining the composite specimens from CTM-SP, some experiments have been performed in the laboratory of Material Engineering Department of Engineering School of São Carlos (University of Sao Paulo - Brazil) (Figure 15). The test machine was an EMIC, which provided the force and displacement data. The strain measurements were carried out by using strain gages and Digital Image Correlation (DIC) technique. In order to perform DIC, the coupons were painted white and black, what was spread to make small marks on the coupons surface. A CANON (EOS 50D) camera was used. It had $400 \mathrm{~mm}$ lens, an aperture of $\mathrm{f} / 4.5$ and a focus distance close to $1.5 \mathrm{~m}$. LED lights were used to illuminate the specimens during the tests, avoiding heat transfer. The images obtained from the CANON camera were analysed by Correlli ${ }^{\text {Q4 }}$ software (Hild \& Roux, 2008). The black marks on the coupon surface were used to measure the displacement fields and strain fields were obtained from displacement fields gradients. 

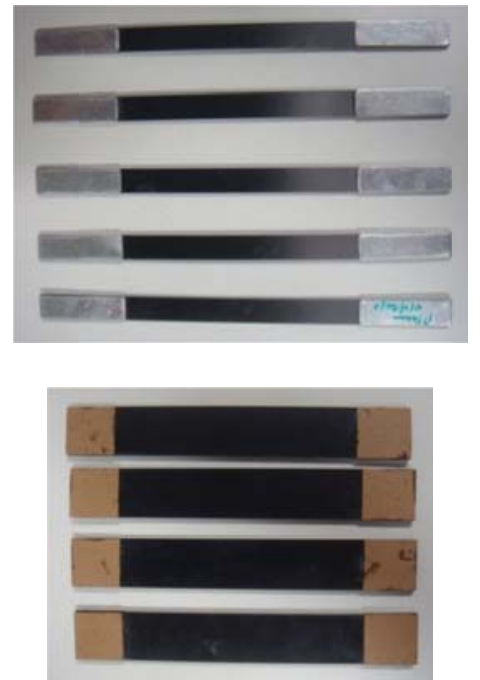

(a)

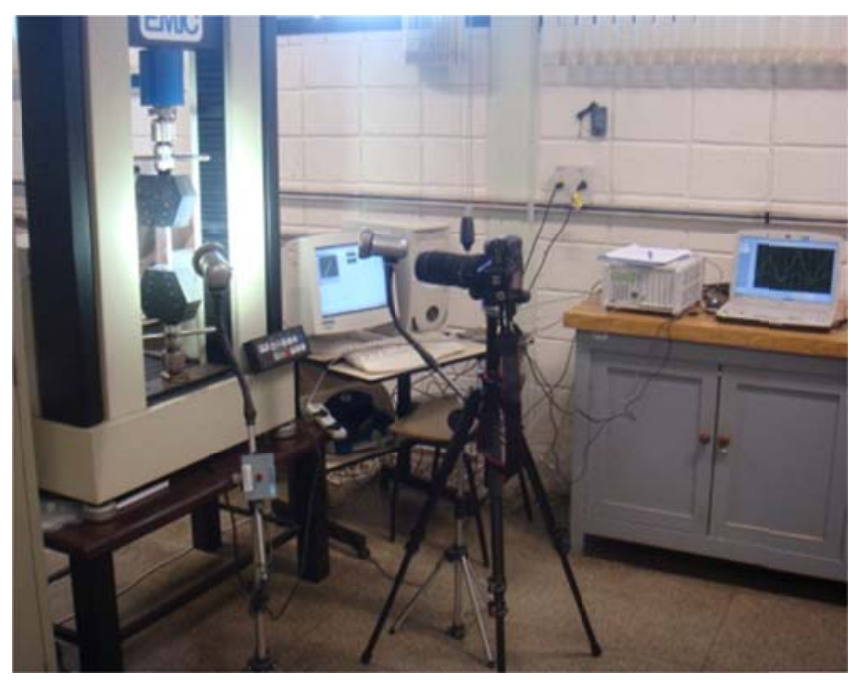

(b)

Figure 15: (a) Flat filament wound coupons; (b) Experimental test set-up at University of São Paulo (Brazil).

Other coupons (Figure 16(a)) were analysed at the Materials Engineering Department of the KU Leuven (Belgium) by using an Instron $^{\mathrm{TM}}$ testing machine (Figure 16(b)), which provides force and displacement. The strains were measured by DIC and strain gages, as well (Figure 17(b)). However, for the experiments carried out in Belgium, the DIC was performed by using the Limess ${ }^{\mathrm{TM}}$ system with VIC2D software to analyse the images.
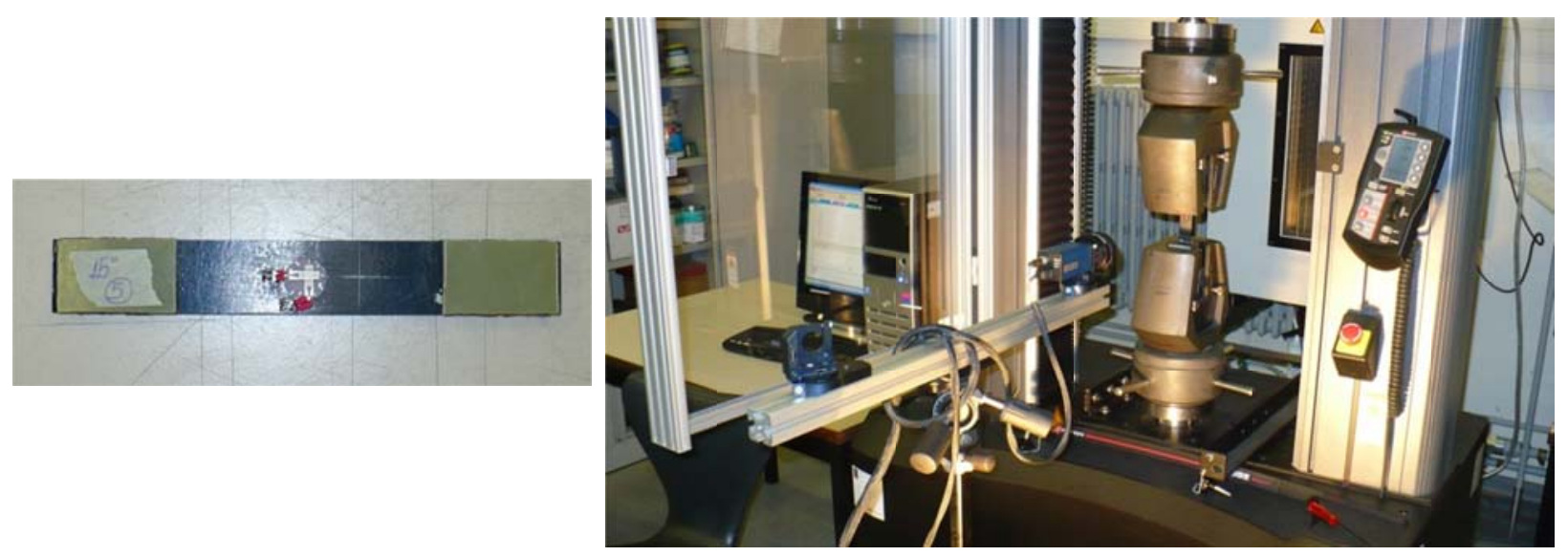

(a)

(b)

Figure 16: (a) Flat filament wound coupons; (b) Experimental test set-up at KU Leuven (Belgium).

Figure 17 shows the paint schema used for DIC, as well as the strain field provided by the software, which is obtained by the displacement field. 


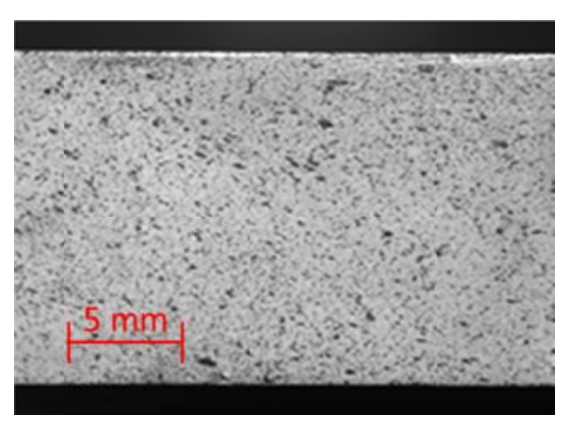

(a)

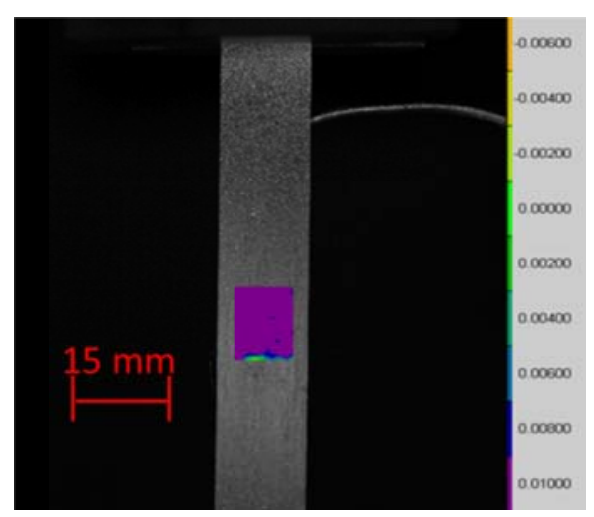

(b)

Figure 17: (a) Coupon painted for image correlation procedure; (b) Image correlation strain field.

Figure 18 shows the stress vs. strain curve obtained by strain gages and DIC for $5^{\circ}$ coupons. Once the differences are small for all cases, only one result of those methods is shown in order to keep the graphics clear.

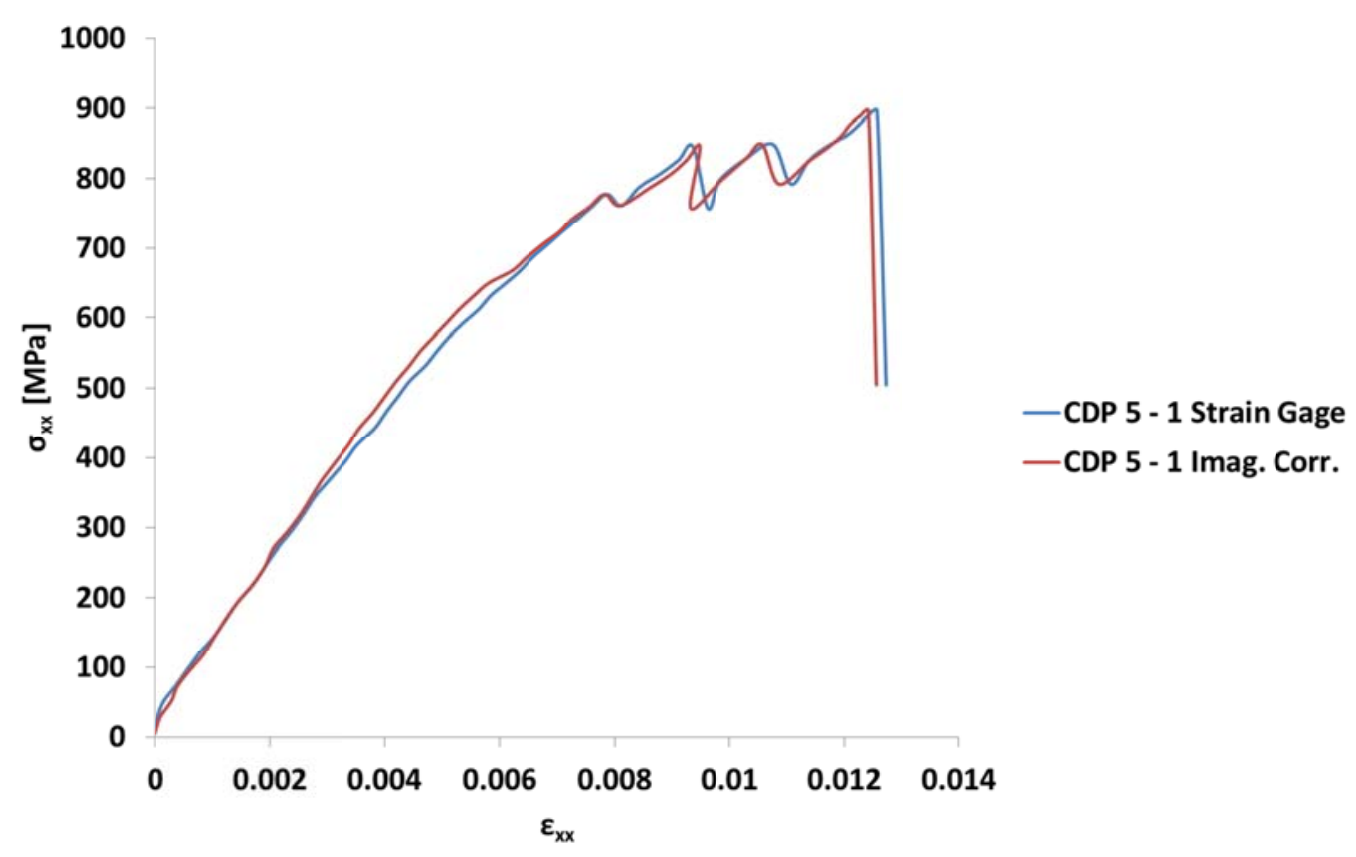

Figure 18: $5^{\circ}$ coupon - monotonic tensile test: Strain gages vs. Image correlation.

\subsubsection{Results and Discussions}

The $0^{\circ}$ coupons experiments results are shown in Figure 19. The results are normalized by the highest tensile stress and strain. One coupon (CDP $0-1)$ has been used for the monotonic test. Based on the material behavior of the coupon (e.g. ductile or fragile) and the strength 
limit, it was possible to determine the load values to perform the cycles. The same test speed $(0.5 \mathrm{~mm} / \mathrm{min})$ has been used for all tests.

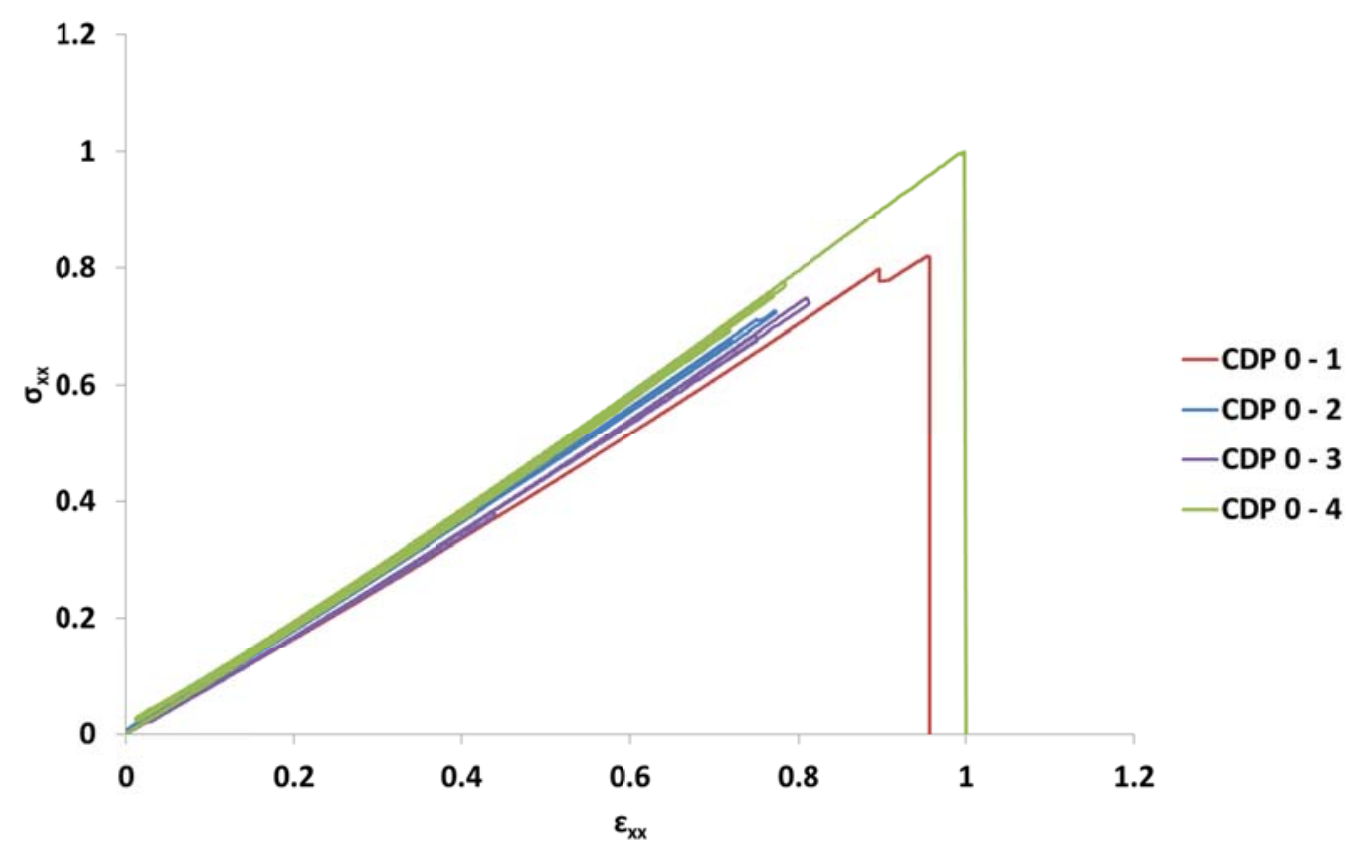

Figure 19: Tensile $0^{\circ}$ coupons - normalized results.

The $0^{\circ}$ coupons presented a linear-elastic behavior, once it has not been detected any stiffness reduction. As expected, unidirectional (UD) $0^{\circ}$ coupons fail in a brittle way. Also, it is possible to observe carbon fiber hardening, but this behavior has not been simulated in this work. The results for all $0^{\circ}$ coupons are shown in Table 8 .

Table 8: Tensile $0^{\circ}$ coupons - normalized results.

\begin{tabular}{cccccc}
\hline Coupon & $\begin{array}{c}\text { Normalized } \\
\text { Stress }\end{array}$ & $\begin{array}{c}\text { Normalized } \\
\text { Strain }\end{array}$ & $\begin{array}{c}\text { Test speed } \\
{[\mathbf{m m} / \mathbf{m i n}]}\end{array}$ & Remarks & Area [mm $\left.\mathbf{m}^{\mathbf{}}\right]$ \\
\hline CDP 0-1 & 0.82 & 0.96 & 0.5 & $\begin{array}{c}\text { Unidirectional strain } \\
\text { gage }\end{array}$ & 15.10 \\
\hline CDP 0-2 & 0.73 & 0.78 & 0.5 & - & 15.23 \\
\hline CDP 0-3 & 0.75 & 0.82 & 0.5 & - & 15.05 \\
\hline CDP 0-4 & 1.00 & 1.00 & 0.5 & - & 15.15 \\
\hline Average & 0.82 & 0.89 & - & - & 15.13 \\
\hline
\end{tabular}


Figure 20 shows the normalized results for $90^{\circ}$ coupons tensile tests. The first test was monotonic in order to know the material behavior, as well as its strength values. Hence, it was possible to plan the limits in all cycles.

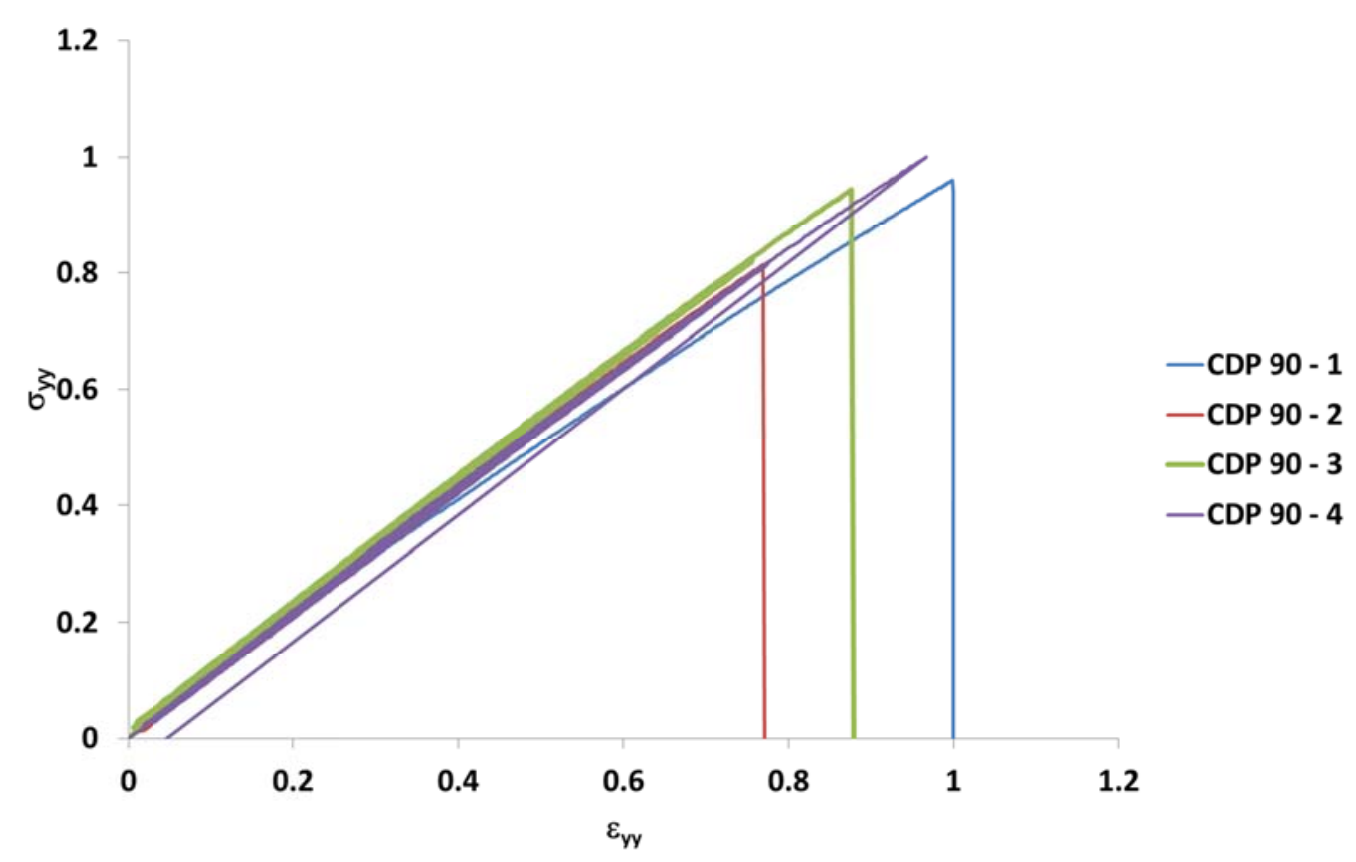

Figure 20: Tensile $90^{\circ}$ coupons - normalized results.

The coupons with fibers at $90^{\circ}$ fail due to matrix damage. These coupons possess a small non-linearity and almost no inelastic strain is observed. As coupons with fibers at $0^{\circ}$, coupons with fibers at $90^{\circ}$ fail in brittle way, also. The results for $90^{\circ}$ coupons are shown in Table 9.

Table 9: Tensile $90^{\circ}$ coupons - normalized results.

\begin{tabular}{cccccc}
\hline Coupon & $\begin{array}{c}\text { Normalized } \\
\text { Stress }\end{array}$ & $\begin{array}{c}\text { Normalized } \\
\text { Strain }\end{array}$ & $\begin{array}{c}\text { Test speed } \\
{[\mathbf{m m} / \mathbf{m i n}]}\end{array}$ & Remark & Area $\left[\mathbf{m m}^{2}\right]$ \\
\hline CDP 90-1 & 0.96 & 1.00 & 0.5 & $\begin{array}{c}\text { Unidirectional strain } \\
\text { gage }\end{array}$ & 52.29 \\
\hline CDP 90-2 & 0.81 & 0.77 & 0.5 & - & 52.10 \\
\hline CDP 90-3 & 0.94 & 0.88 & 0.5 & - & 52.35 \\
\hline CDP 90-4 & 1.00 & 0.97 & 0.5 & - & 52.15 \\
\hline CDP 90-5 & 0.93 & 0.90 & 0.5 & - & 52.55 \\
\hline Average & 0.96 & 1.00 & - & - & 52.29 \\
\hline
\end{tabular}


As shown in Figure 12, the ply orientation affects both the composite behavior and failures. At this point, it is important to comment some aspects related to off-axis coupons tests.

The first important remark regards the difficulty to apply loading only to longitudinal direction. In fact, non-zero components in the bending-stretching coupling matrix (laminate stiffness matrix B) take place, when tensile tests with usual grips are carried out. Hence, it is not possible to apply only axial loads, because unknown shear stress is applied due to the end clamped condition (Figure 21). Thus it is not possible to use these results to measure the elastic properties.

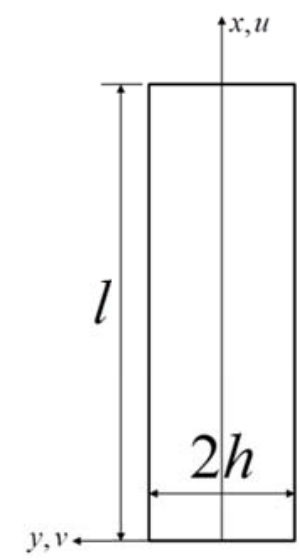

Figure 21: Coupon geometry.

Herakovich (1998) showed a solution, which is based on Theory of Elasticity, to evaluate the effect of shear stress on the results. Initially, the lamina is assumed to be in plane stress state under a far loading field. Considering that the sides of coupons are stress free, boundary conditions are given as follows:

$$
\begin{aligned}
& \sigma_{y}(x, \pm h)=0 \\
& \tau_{x y}(x, \pm h)=0
\end{aligned}
$$

where $\mathrm{h}$ is the half coupon width. The approximate displacement boundary conditions are:

$$
v(0,0)=\frac{\partial u(0,0)}{\partial y}=0
$$




$$
\begin{gathered}
v(l, 0)=\frac{\partial u(l, 0)}{\partial y}=0 \\
u(0,0)=0 \\
u(l, 0)=\varepsilon_{x}^{0} l
\end{gathered}
$$

where $\varepsilon_{x}^{0}$ is the far field axial strain. Also, the boundary conditions only constrain the displacement at the center line of the coupons ends.

The shear stress is assumed to be constant along the length ( $\mathrm{x}$ direction - Figure 21) of the coupon and it is an unknown function of $y$.

$$
\begin{gathered}
\tau_{x y}=f_{1}(y) \\
\frac{\partial \sigma_{x x}}{\partial x}+\frac{\partial \tau_{x y}}{\partial y}=0 \\
\frac{\partial \sigma_{y y}}{\partial y}+\frac{\partial \tau_{y x}}{\partial x}=0
\end{gathered}
$$

Integrating the plane stress equilibrium equations (EQ. (58) and EQ.(59)) and using EQ. (57), the expressions for the normal stresses components are:

$$
\begin{gathered}
\sigma_{x}=-x f_{1}^{\prime}(y)+f_{2}(y) \\
\sigma_{y}=g(x)
\end{gathered}
$$

where $f_{2}(y)$ and $g(x)$ are functions of their respective arguments. Using the previous results with the compatibility equations (EQ.(62)) leads to the third-order differential equation (EQ.(63)).

$$
\begin{gathered}
2 \varepsilon_{x y, y x}=\varepsilon_{x x, y y}+\varepsilon_{y y, x x} \\
-\bar{S}_{11} x f_{1}^{\prime \prime}(y)+\bar{S}_{11} x f_{2}^{\prime \prime}(y)+2 \bar{S}_{16} x f_{1}^{\prime \prime}(y)+\bar{S}_{22} g^{\prime \prime}(x)=0
\end{gathered}
$$


where $\bar{S}_{11}, \bar{S}_{22}$ and $\bar{S}_{16}$ are terms of the laminate compliance matrix. The solution for this partial differential equation, which satisfies the boundary conditions, is:

$$
\begin{gathered}
f_{1}(y)=C_{0}\left(y^{2}-h^{2}\right) \\
f_{2}(y)=-2 \frac{\bar{S}_{16}}{\bar{S}_{11}} C_{0} y^{2}+C_{1} y+C_{2} \\
g(x)=0
\end{gathered}
$$

The stresses are:

$$
\begin{gathered}
\sigma_{x}=-2 \frac{\bar{S}_{16}}{\bar{S}_{11}} C_{0} y^{2}+C_{1} y+C_{2}-2 C_{0} x y \\
\sigma_{y}=0 \\
\tau_{x y}=C_{0}\left(y^{2}-h^{2}\right)
\end{gathered}
$$

where $C_{0}, C_{1}$ and $C_{2}$ are unknown integration constants. These constants may be determined by using both the boundary conditions (EQ. (53) to EQ. (56)) and the straindisplacement relations shown below.

$$
\begin{gathered}
\varepsilon_{x}=\frac{\partial u}{\partial x} \\
\varepsilon_{y}=\frac{\partial v}{\partial y} \\
\gamma_{x y}=\frac{\partial u}{\partial y}+\frac{\partial v}{\partial x}
\end{gathered}
$$

The strains in terms of stress, considering $\sigma_{y}=0$, are:

$$
\varepsilon_{x}=\bar{S}_{11} \sigma_{x}+\bar{S}_{16} \tau_{x y}
$$




$$
\begin{aligned}
& \varepsilon_{y}=\bar{S}_{12} \sigma_{x}+\bar{S}_{26} \tau_{x y} \\
& \gamma_{x y}=\bar{S}_{16} \sigma_{x}+\bar{S}_{66} \tau_{x y}
\end{aligned}
$$

The integration of EQ.(70) using EQ.(67), EQ.(69) and EQ.(73) provides the $u$ displacement. The integration of EQ. (71) using EQ.(67), EQ. (69) and EQ.(74) gives the v displacement. By using some steps explained by Herakovich (1998), it is possible to determine the integration constants. Regarding the displacements along the centerline, $y=0$, (Figure 21), it is obtained:

$$
\begin{gathered}
u=\left(\bar{S}_{11} C_{2}-\bar{S}_{16} C_{0} h^{2}\right) x \\
v=\frac{\bar{S}_{11} C_{0}(2 x-l)(x-l) x}{6}
\end{gathered}
$$

Thus, the stress $\sigma_{x}$ in the center-line is given by:

$$
\sigma_{x}=\frac{\left[6\left(\frac{\bar{S}_{66}}{\bar{S}_{11}}\right)+\left(\frac{l}{h}\right)^{2}\right] \varepsilon_{x}^{0}}{\bar{S}_{11}\left\{6\left[\frac{\bar{S}_{66}}{\bar{S}_{11}}-\left(\frac{\bar{S}_{16}}{\bar{S}_{11}}\right)^{2}\right]+\left(\frac{l}{h}\right)^{2}\right\}}
$$

And, the shear stress is given by:

$$
\tau_{x y}=\frac{-6\left(\frac{\bar{S}_{16}}{\bar{S}_{11}}\right)}{6\left(\frac{\bar{S}_{66}}{\bar{S}_{11}}\right)+\left(\frac{l}{h}\right)^{2}} \sigma_{x}
$$

Once the strains have been measured by using either strain gages or DIC, it is possible to verify the effect of the shear stress in the results. 
Also, from EQ. (78), it is verified that if the coupon has a large length $(I)$ or/and a small width $(h)$, the effect of shear stress is smaller. Thus, shear stress is negligible for the ratio $l / h$ around 15 (Herakovich, 1998).

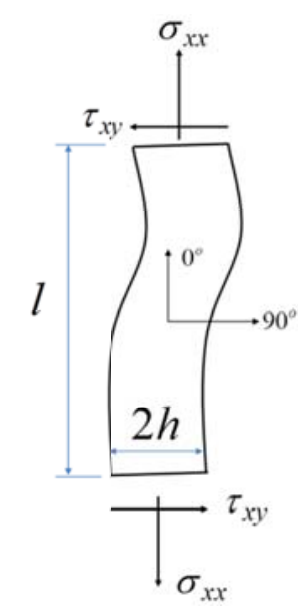

Figure 22: Applied loads to off-axis coupons during tensile tests by using usiual grips.

The effect of curvature on the coupons for tensile tests can be neglected since the machine grips straight the coupons. Also, the grips are much stiffer than the coupons and the residual stress is low, i.e. lower than $1 \%$ of the $\mathrm{Y}_{\mathrm{T}}$, which is the lowest material strength value.

The off-axis $5^{\circ}$ coupons tests results are presented in Figure 23. As previously mentioned first of all, a monotonic test was performed, and then the cycle tests were planned. For these tests, the effect of damage causes inelastic strain as well as reduction of the stiffness. 


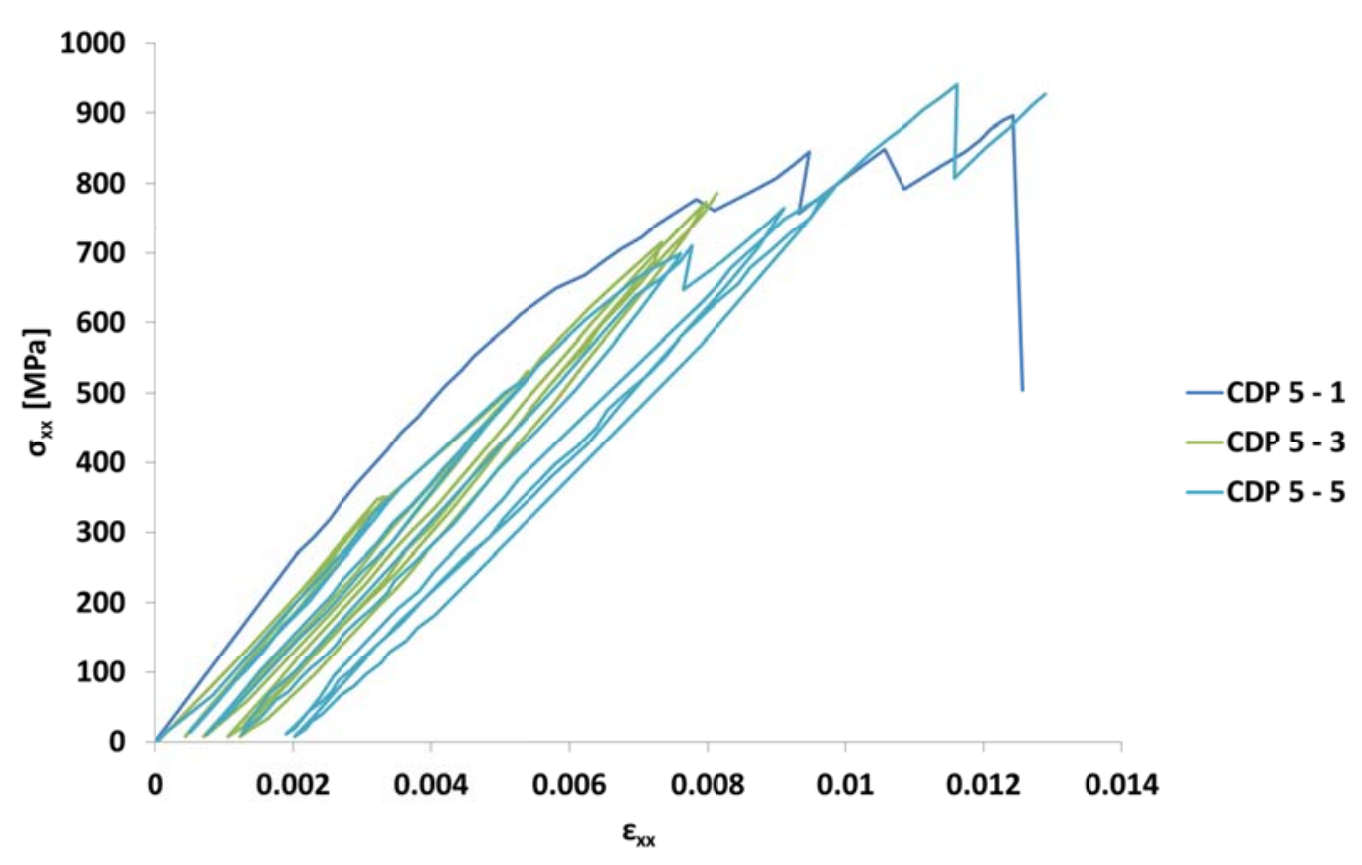

Figure 23: Tensile $5^{\circ}$ coupons results.

Figure 24 shows the results for EQ.(78) and EQ.(79) as well as the monotonic $5^{\circ}$ coupons experimental results, which show that the shear stress is rather low and, the measured $\sigma_{x}$ has not been affected by the $\tau_{x y}$. It is important to notice that the equations (78) and (79) are valid only for the linear part of the experimental curve. 


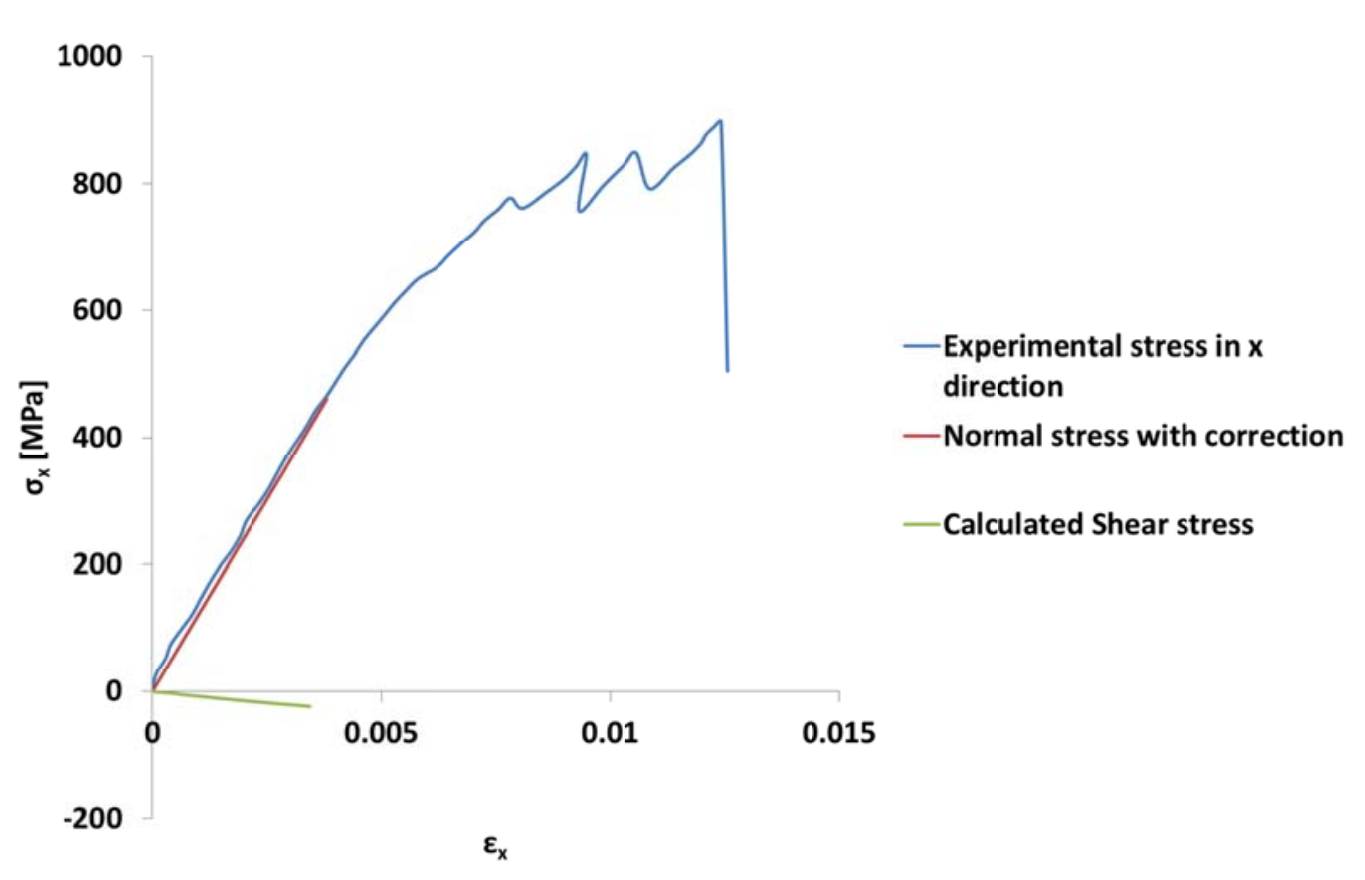

Figure 24: Comparison of experimental results and boundary conditions corrections (EQ.(78) and EQ.(79)) results for $5^{\circ}$ coupons.

With these previous results, the stress tensor has been rotated to ply direction, according to EQ. (80) and assuming $\sigma_{y}=\tau_{x y}=0$.

$$
\left[\begin{array}{ll}
\sigma_{11} & \tau_{12} \\
\tau_{21} & \sigma_{22}
\end{array}\right]=\left[\begin{array}{cc}
\cos \theta & \sin \theta \\
-\sin \theta & \cos \theta
\end{array}\right] \cdot\left[\begin{array}{cc}
\sigma_{x} & \tau_{x y} \\
\tau_{y x} & \sigma_{y}
\end{array}\right] \cdot\left[\begin{array}{cc}
\cos \theta & -\sin \theta \\
\sin \theta & \cos \theta
\end{array}\right]
$$

With the ply orientation stress tensor, the damage variables $d_{2}$ and $d_{6}$ can be obtained as already shown in the previous chapter.

Angle-ply laminates do not have the same characteristic presented by off-axis coupons. The results of tensile test for angle-ply $67.5^{\circ}$ coupons are shown in Figure 25. 


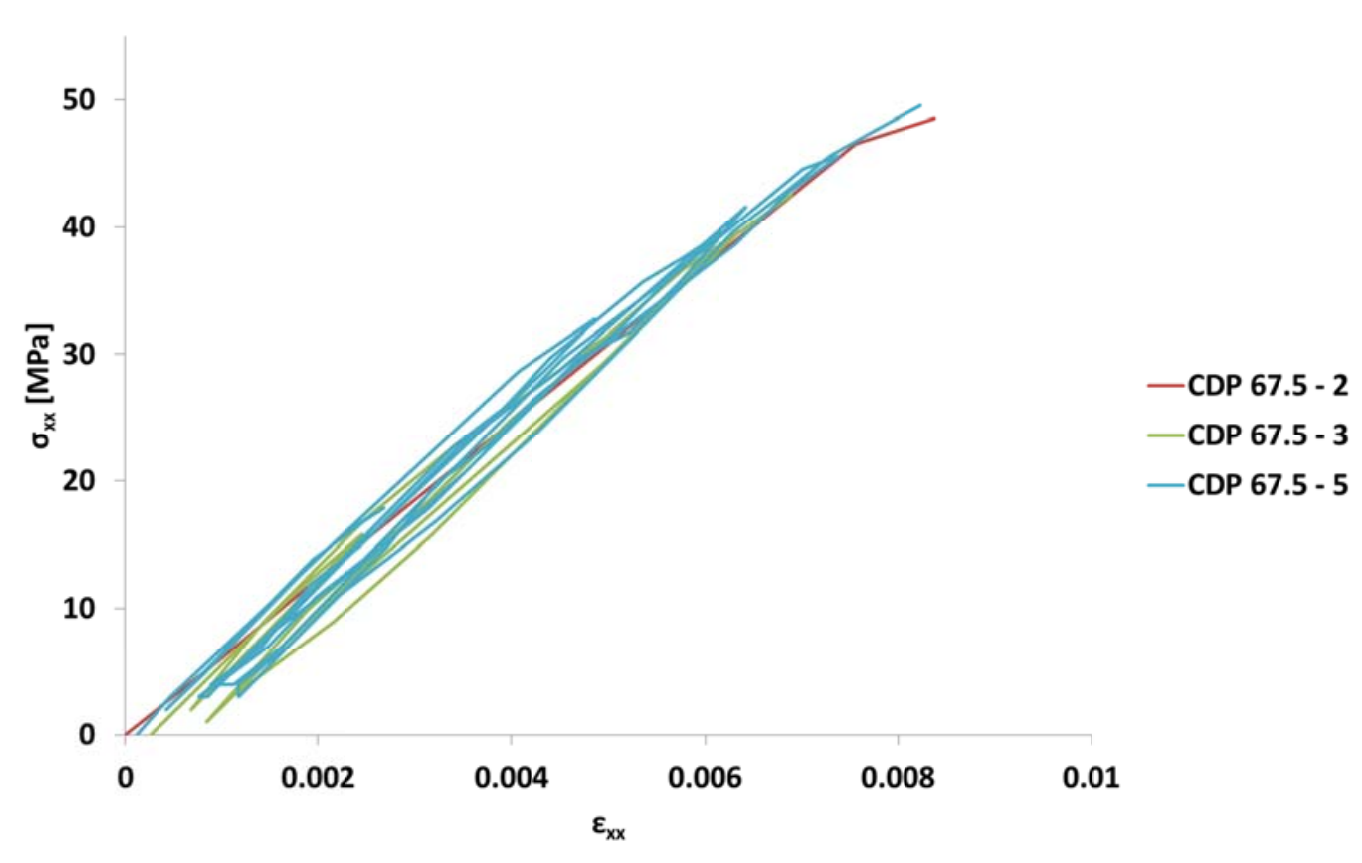

Figure 25: Tensile $67.5^{\circ}$ coupons results.

Again, these tests allow evaluating how the damage variables $d_{2}$ and $d_{6}$ evolve. It is important to verify that angle-ply $67.5^{\circ}$ laminate fails under stress value lower than coupons with fibers at $90^{\circ}$. This result indicates that damage evolves faster due to the shear influence damage.

\subsubsection{Tensile Tests Conclusions}

Both the $0^{\circ}$ and $90^{\circ}$ coupons tensile tests provide the strength parameters as well as the elastic properties. As expected, coupons with fibers at $0^{\circ}$ showed a linear elastic behavior with brittle fracture. The coupons with fibers at $90^{\circ}$ possess a non-linear behavior allowing investigating how the damage variable $\mathrm{d}_{2}$ evolves without the influence of shear stress.

To investigate the stresses coupling, the usage of $5^{\circ}$ off-axis coupons was very strategic due to some additional complexities shown by test boundary conditions and off-axis characteristics. Once the effect of shear stress has been considered, it is possible to study the damage variables $d_{2}$ and $d_{6}$ and how they interact. The off-axis results have not been used to obtain any elastic parameters.

Angle-ply $67.5^{\circ}$ laminates also allow evaluating the effect of shear damage $\left(d_{6}\right)$ in the matrix damage $\mathrm{d}_{2}$, without the boundary condition issues presented by the off-axis coupons. 
Finally, the effect of coupons curvature has not been modeled for the tensile tests, once it is very small. In fact, the test machine grips straight the coupons and the stresses created by this phenomenon are very small.

\subsubsection{COMPRESSION TESTS}

Compression tests are rather complicated to perform, since buckling is very difficult to avoid. In order to minimize the buckling phenomenon, the coupons span must be small enough and still have sufficient length to allow measuring the strains without Saint-Venant effects.

Despite the negligible effect of coupons curvature for the tensile tests, this initial curvature affects the compression results and other non-acceptable failure modes may occur. For example, it is possible to identify end-crushing, decohesion of tab adhesive, failure inside the tab area and delaminations (ASTM D3410, 2003), which are unacceptable failure modes for compression tests (Figure 26).

\subsubsection{Materials and Methods}

As in the tensile tests, the coupons for compression tests have been produced by filament winding process and, the almost flat composite laminate plates have been manufactured (with radius around $7657 \mathrm{~mm}$ ) by using a parallelepiped shape mandrel. Again, the plates have been cured in a controlled oven. After that, the almost flat plates have been cut into coupons, following the dimensions provided by the ASTM D3410 (2003) standards.

The dimensions for $0^{\circ}, 90^{\circ}, 30^{\circ}$ and $15^{\circ}$ compression test coupons are shown in Table 5. Table 6 shows where the tests were performed and why. As previously explained for tensile tests, the off-axis orientation has been chosen in order to study different failure modes and how shear stress $\left(\tau_{12}\right)$ and normal stress $\left(\sigma_{2}\right)$ interact during the damage process.

Although the dimensions have been according to the standards, the compression tests of off-axis coupons have also been submitted to unknown shear stress $\left(\tau_{x y}\right)$ due to non-zero terms in the bending-stretching coupling matrix (laminate stiffness matrix B). Despite knowing that the $h / l$ ratio (EQ.(48) and EQ.(49)) may reduce the influence of shear stress, the span in compression tests must be as small as possible to avoid buckling. 

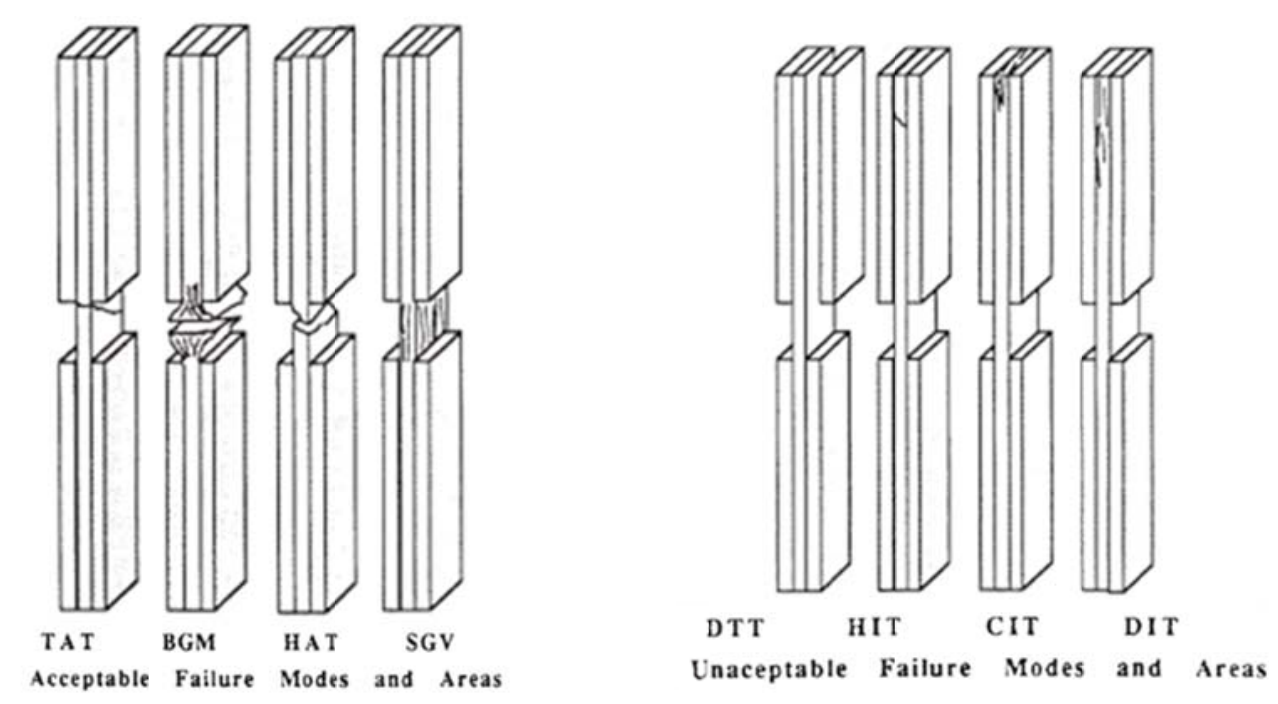

\begin{tabular}{|lc|}
\multicolumn{2}{c}{ First Character } \\
\hline Failure Mode & Code \\
\hline Angled & $\mathrm{A}$ \\
Brooming & $\mathrm{B}$ \\
end-Crushing & $\mathrm{C}$ \\
Delamination & $\mathrm{D}$ \\
Euler buckling & $\mathrm{E}$ \\
tHrough-thickness & $\mathrm{H}$ \\
Kink bands & $\mathrm{K}$ \\
Lateral & $\mathrm{L}$ \\
Muiti-mode & $\mathrm{M}(\mathrm{xyz})$ \\
long--Splitting & $\mathrm{S}$ \\
Transverse shear & $\mathrm{T}$ \\
cXplosive & $\mathrm{X}$ \\
Other & $\mathrm{O}$ \\
\hline
\end{tabular}

Second Character

\begin{tabular}{|lc|}
\hline Failure Area & Code \\
\hline Inside grip/tab & I \\
At grip/tab & A \\
Gage & G \\
Multiple Areas & M \\
Tab adhesive & T \\
Various & V \\
Unknown & U \\
\hline
\end{tabular}

Third Character

\begin{tabular}{|lc|}
\hline Failure Location & Code \\
\hline Bottom & B \\
Top & $\mathrm{T}$ \\
Left & $\mathrm{L}$ \\
Right & $\mathrm{R}$ \\
Middle & $\mathrm{M}$ \\
Various & $\mathrm{V}$ \\
Unknown & $\mathrm{U}$ \\
\hline
\end{tabular}

Figure 26: Acceptable and unacceptable failure modes for compression tests (ASTM D3410, 2003).

Thus, the influence of shear stress is stronger for off-axis compression tests than for tensile tests. Therefore it is not possible to use off-axis coupons to obtain the elastic properties, but it still allows studying the coupling between $\tau_{12}$ and $\sigma_{22}$ in the damage process.

As in the tensile tests, all compression tests data were obtained by using strain-gages and DIC. Figure 27(a) shows the device used for this test and Figure 27(b) shows the coupons for compression tests. 


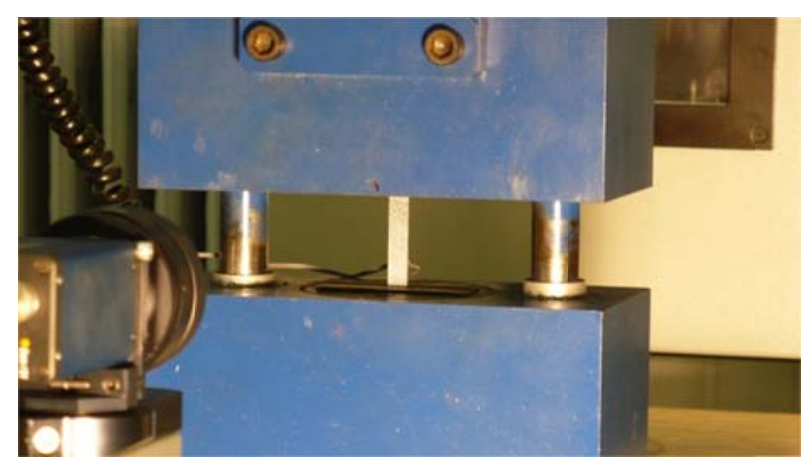

(a)

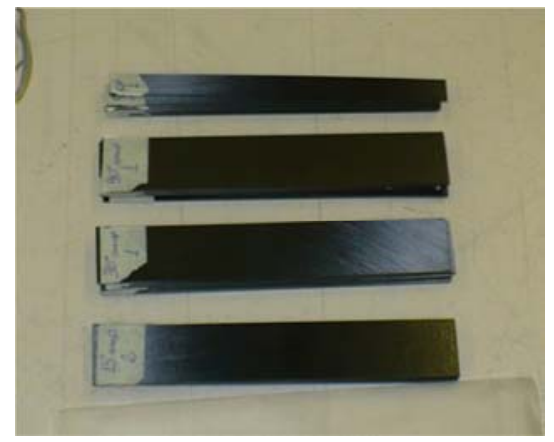

(b)

Figure 27: (a) Compression test device; (b) Compression coupons.

As commented before, buckling phenomenon has been a critical problem mainly for coupons with fibers at $30^{\circ}$ off-axis and $90^{\circ}$ (see Figure 28). It is important to mention that buckling has not been verified for other investigated orientations.

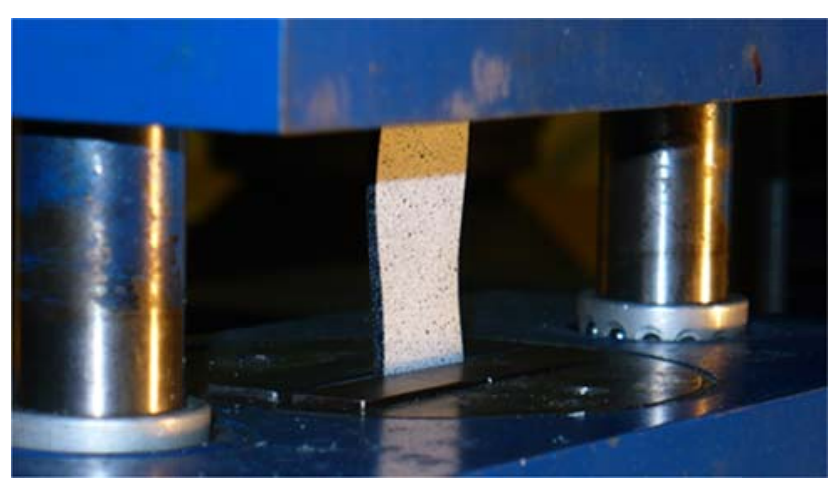

Figure $28: 30^{\circ}$ coupon - Buckling phenomenon.

\subsubsection{Results and Discussions}

Strength values for $0^{\circ}$ coupons have also been obtained from compression tests. The test results have been presented in normalized format (Figure 29) because the material data are classified. In order to keep graphic clearness, only DIC data are shown.

The buckling critical load is highly dependent on the boundary conditions. To verify the upper and lower critical load, the coupon is modeled as a simple column. For the lower critical load, the coupon is pinned on both ends and, for upper limit (EQ. (81)), the coupon is clamped on both ends (EQ.(82)). 


$$
\begin{aligned}
& P_{C R}=\frac{\pi^{2} E_{x}}{12}\left(\frac{t}{L}\right)^{2} \\
& P_{C R}=\frac{\pi^{2} E_{x}}{3}\left(\frac{t}{L}\right)^{2}
\end{aligned}
$$

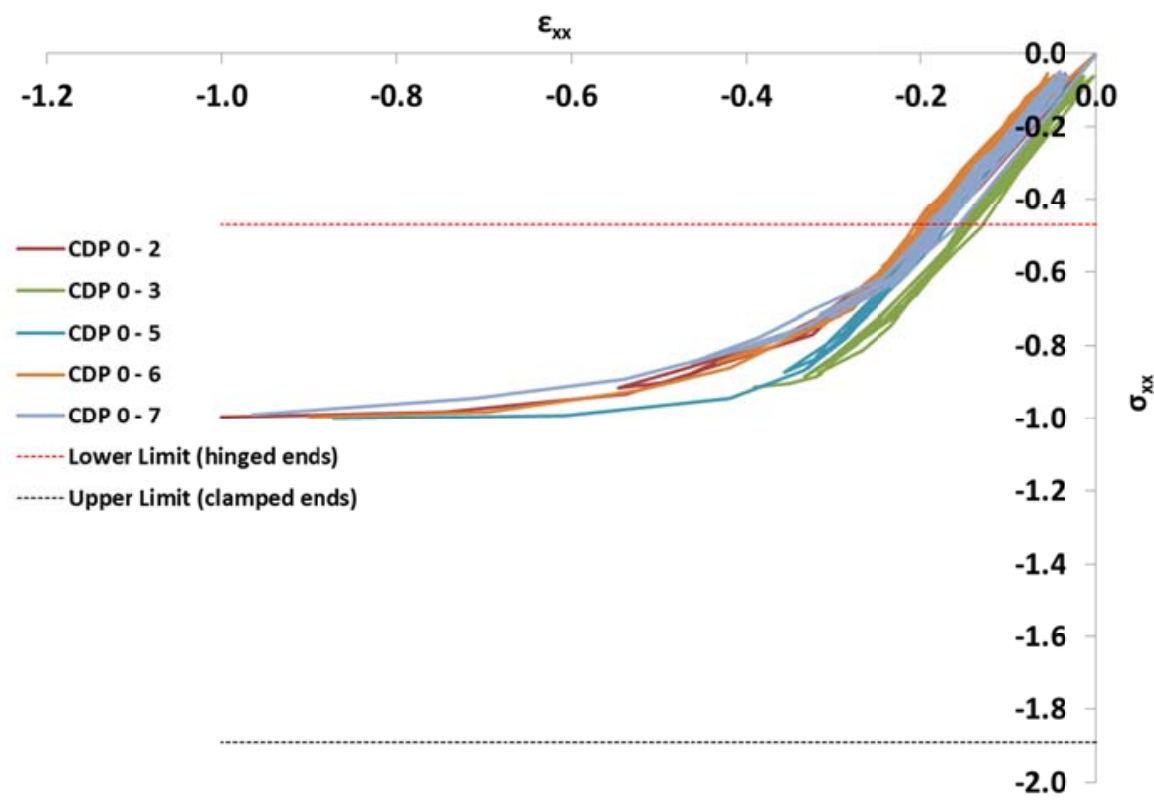

Figure 29: Compression $0^{\circ}$ coupons - normalized results.

The compression of coupons with fibers at $0^{\circ}$ showed a non-linear elastic behavior. The experimental critical loads are between the theoretical upper and lower limits. The lower limit ( 0.47 of the experiments maximum load) represents the pinned ends conditions, which are not representative of the experiments. The upper limit load (1.89 of the experiments maximum load) boundary conditions (clamped on both ends) are the most representative of the experiments. In fact, the real boundary conditions are between both ideal cases, but closer to the clamped ends.

Also the higher load obtained in the compression test is quite lower than the strength values for $0^{\circ}$ coupon tensile strength, i.e. lower than $20 \%$ of tensile strength. It may be an evidence of the coupon curvature effect the compression results, despite the coupons are straightened by the test machine fixture. In other words, the straightened coupons have a beam-column behavior. 
On the other hand, as commented before, most of $0^{\circ}$ coupons failed inside the grips or showed end-crushing. Only the coupon defined as CDP 0-3 (Figure 29) showed an acceptable failure mode, but its behavior is rather similar to the other coupons. Another explanation could be related to fiber misalignment during the manufacturing process, once only small difference in the orientation could affect the results significantly (Yokozeki, Ogasawara, \& Ishikawa, 2006). Table 10 shows the results and other test remarks. In fact, for the material model, the elastic properties have been obtained by the tensile tests and not by the compression ones.

Table 10: Compression $0^{\circ}$ coupons - normalized results.

\begin{tabular}{ccccc}
\hline Cou pon & $\begin{array}{c}\text { Normalized } \\
\text { Stress }\end{array}$ & $\begin{array}{c}\text { Normalized } \\
\text { Strain }\end{array}$ & Test speed $[\mathbf{m m} / \mathbf{m i n}]$ & Area [ $\left.\mathbf{m m}^{2}\right]$ \\
\hline CDP 0-1 & -0.85 & -0.43 & 0.5 & 22.52 \\
\hline CDP 0-2 & -1.00 & -1.00 & 0.5 & 20.36 \\
\hline CDP 0-3 & -0.91 & -0.39 & 0.5 & 21.45 \\
\hline CDP 0-4 & -0.75 & -0.30 & 0.5 & 22.58 \\
\hline CDP 0-5 & -1.00 & -0.87 & 0.5 & 21.41 \\
\hline CDP 0-6 & -1.00 & -0.90 & 0.5 & 21.91 \\
\hline CDP 0-7 & -0.99 & -0.97 & 0.5 & 22.05 \\
\hline Average & -0.93 & -0.69 & - & 21.63 \\
\hline
\end{tabular}

Compression tests of specimens with fibers at $90^{\circ}$ showed to be very difficult to perform, once buckling took place for almost all coupons. Two experimental normalized results are shown in Figure 30 as well as an upper (EQ.(82)) and lower (EQ.(81)) critical buckling load. The lowest limit buckling load is 0.31 of the experiments maximum lad and the highest limit is 1.24. In this case, the experiments results are closer to the highest limit than those for compression $0^{\circ}$ coupons.

The strength values are rather low compared to literature values for compression. Figure 30 only shows the two best results out of seven tests. Again, the initial coupon curvature straightened by the machines fixtures makes the coupon behave as a beam-column and it buckles with a lower load than for a straight coupon one. Although the strength values are low, these results have been used for the model development.

It is important to observe that for $90^{\circ}$ compression, the behavior is similar to $0^{\circ}$ coupons, i.e. non-linear elastic behavior and almost no inelastic strains have been verified. This 
behavior is due to cracks closures under compression (Allix, Ladevèze, \& Vittecoq, 1994), and there is no shear stress, which creates matrix damage.

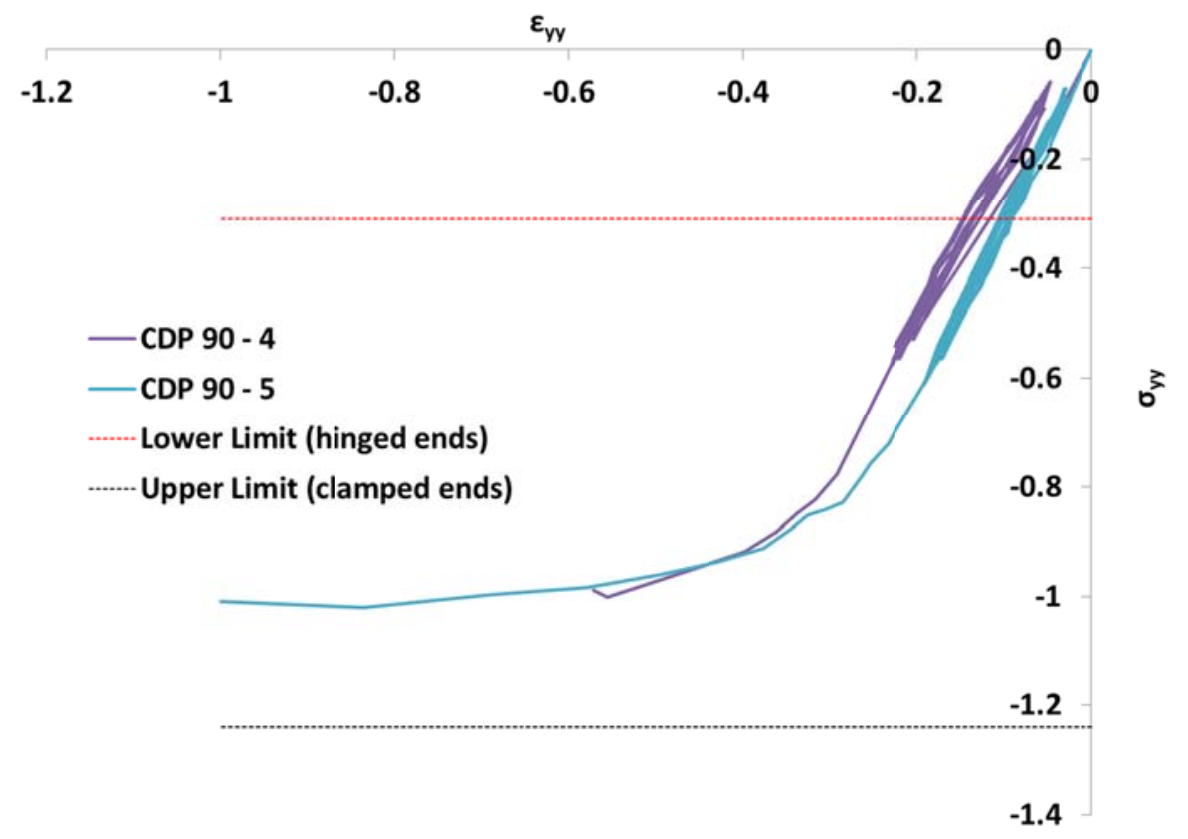

Figure 30: Compression $90^{\circ}$ coupons - normalized results.

Table 11 shows the results and other test remarks for $90^{\circ}$ coupons under compression loads.

Table 11: Compression $90^{\circ}$ coupons - normalized results.

\begin{tabular}{ccccc}
\hline Coupon & $\begin{array}{c}\text { Normalized } \\
\text { Stress }\end{array}$ & $\begin{array}{c}\text { Normalized } \\
\text { Strain }\end{array}$ & Test speed $[\mathbf{m m} / \mathbf{m i n}]$ & Area $\left[\mathbf{m m}^{2}\right]$ \\
\hline CDP 90-4 & -1.00 & -1.00 & 0.5 & 56.30 \\
\hline CDP 90-5 & -0.85 & -0.57 & 0.5 & 55.20 \\
\hline Average & -0.93 & -0.79 & - & 55.75 \\
\hline
\end{tabular}

As previously mentioned, when off-axis compression or tensile test are carried out in longitudinal direction using usual grips, it is not possible to apply only $\sigma_{x}$, but also shear stress. By using EQ.(78) and EQ.(79), it is possible to predict $\tau_{x y}$ value (Figure 31). In this case, due to smaller span and fiber orientation, the effect of shear stress $\tau_{x y}$ is higher than for $5^{\circ}$ coupons under tensile loads. 


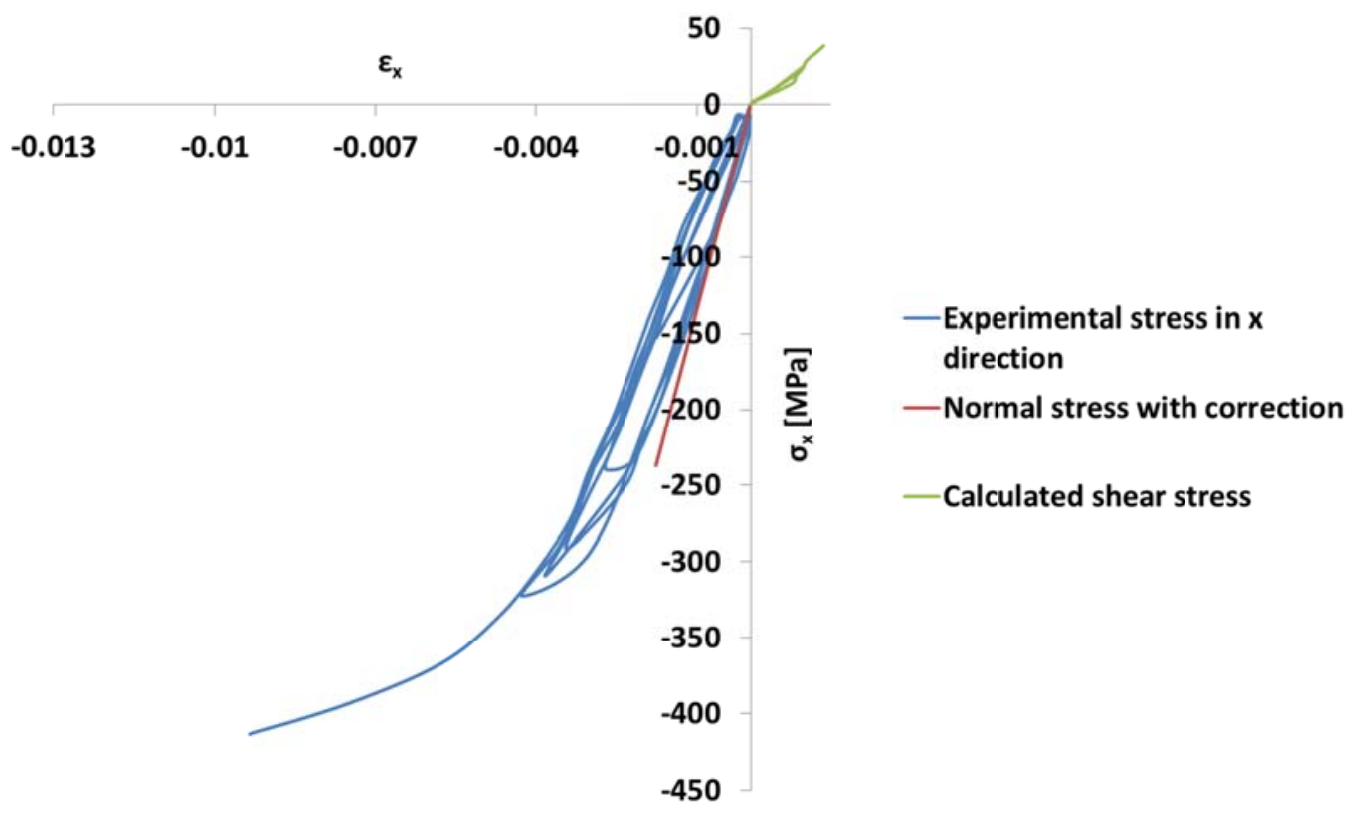

Figure 31: Comparison of experimental results and boundary conditions corrections results for $15^{\circ}$ coupons.

The experimental results for compression $15^{\circ}$ coupons are shown in Figure 32. Only the DIC data and the best results are presented in order to keep figure clearness. The lower critical load limit as well as the upper critical load limits are also shown.

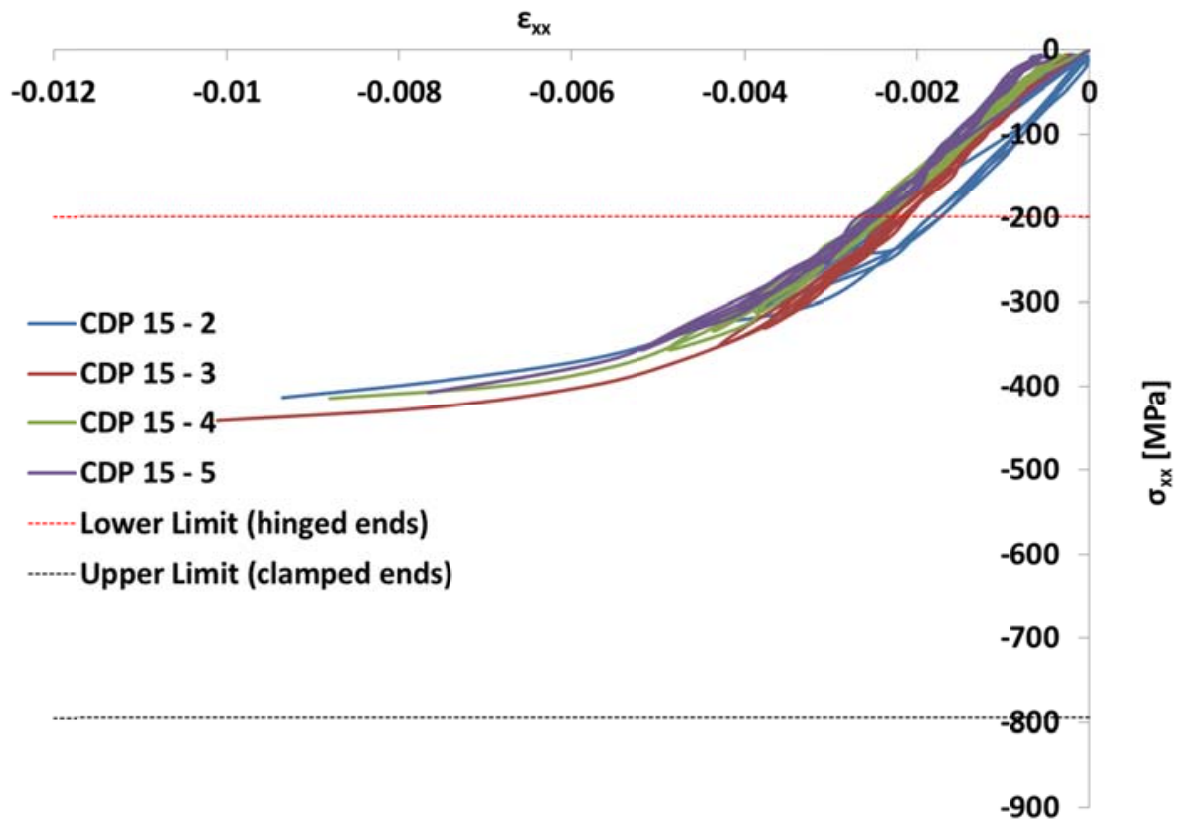

Figure 32: Compression $15^{\circ}$ coupons - results. 
It is important to verify that the compression strength value for coupons with fibers at $15^{\circ}$ is rather close to the results obtained for $0^{\circ}$ coupons. These experiments showed that the results were closer to the lower limit as for $0^{\circ}$ coupons under compression tests.

These compression tests have not been used for obtaining any elastic properties, but for analyzing the damage process. To proceed with damage evolution analyses, the stress tensor in material coordinate has been rotated to ply orientation by using EQ.(80). Thus, it becomes possible to investigate how $\mathrm{d}_{2}$ and $\mathrm{d}_{6}$ evolve and interact. Also, these results have been used to evaluate the material model.

Figure 33 shows the boundary conditions corrections for $30^{\circ}$ coupons. Based on the EQ.(78) and EQ.(79), it is possible to have a prediction of $\tau_{x y}$ for $30^{\circ}$ off-axis coupons under compression (Figure 33).

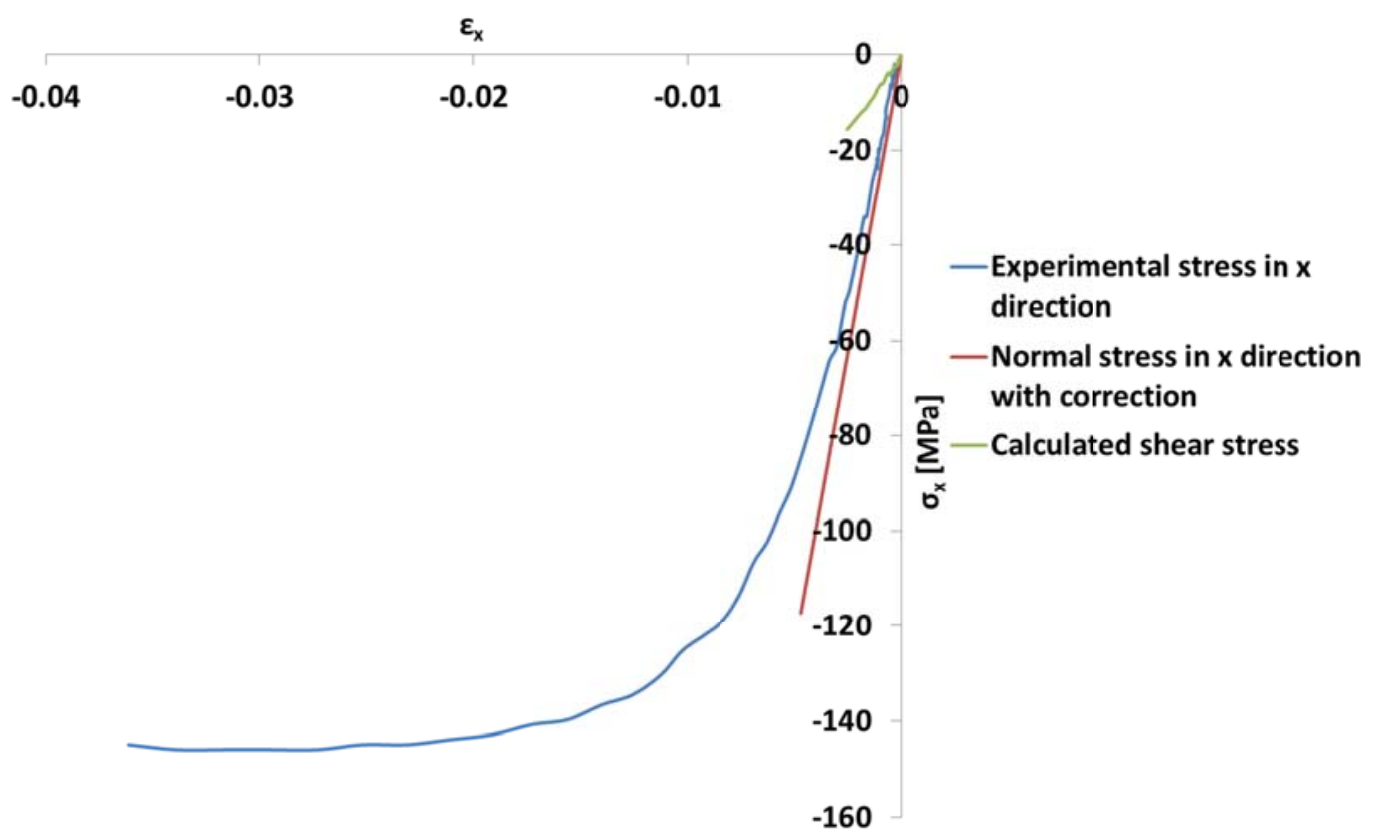

Figure 33: Comparison of experimental results and boundary conditions corrections results for $30^{\circ}$ coupons.

The compression of $30^{\circ}$ coupons results are shown in Figure 34. For this orientation, the failure mode was buckling, what is represented by the flat part of the response curve (around $120 \mathrm{MPa}$ ). Also, $30^{\circ}$ coupons presented inelastic strains caused by damage in the polymer matrix.

In this case, only the buckling lower limit is shown in Figure 34, however the highest limit is equal to $539 \mathrm{MPa}$. In this case, most of coupons buckle even at loads lower than the 
theoretical prediction. The reason for this behavior is that almost all flat coupons become straight when set by the machine fixture and a bending moment is created. Thus the coupons show a beam-column behavior.

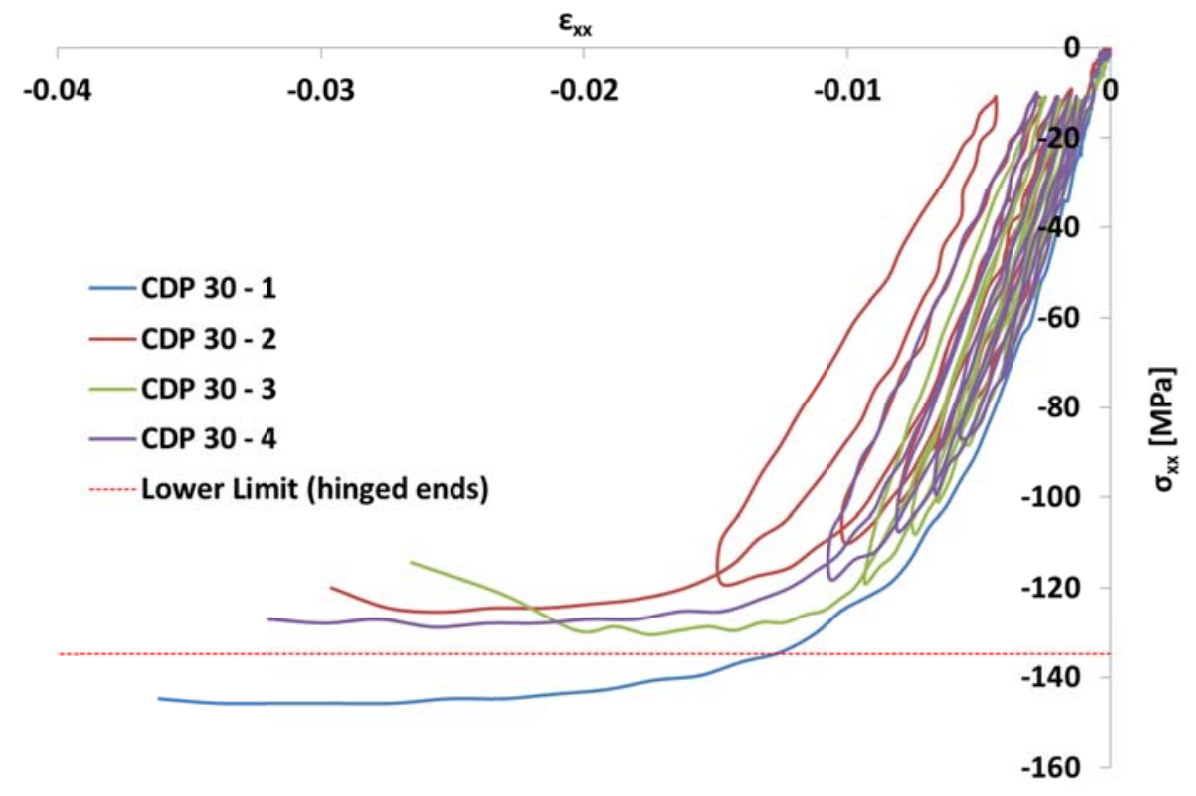

Figure 34: Compression $30^{\circ}$ coupons - results.

\subsubsection{Compression Tests Conclusions}

The compression tests are very complex due to a structural instability and some unacceptable failure modes, e.g. end-crushing. Therefore, the compression tests have been used to understand the damage process and to investigate how the damage variables interact with each other for the material model proposal.

The maximum stress value obtained by the compression tests of $0^{\circ}$ and $90^{\circ}$ coupons have been lower than the results found in the literature for a similar material. Also the experiments maximum stresses have been between lower and upper limits for critical buckling loads. Moreover the results for the coupons are closer to the lowest limit and it is an indication that the buckling load decreases due to the bending moment created by the straightening of almost all the flat coupons, when they are set by the machine fixtures. 


\subsubsection{SHEAR TESTS}

The measurement of composite shear characteristics can be performed either by using coupons with fibers at $\pm 45^{\circ}$, following the orientations provided by ASTM D3518 (2007), or by using a rail test, which has been described by ASTM D4255 (2007).

For the shear elastic properties and strength values, it was used the same values identified by Tita (2003), because some issues related to filament winding manufacturing process does not allow producing coupons as described by ASTM D3518. In fact, the process for manufacturing quasi-flat coupons enforces some limits for the winding angle and the $\pm 45^{\circ}$ can be reached only in a small part of the flat mandrel. Therefore only coupons, whose sizes are smaller than those recommended by standard, could be produced by winding process and the elastic properties and strength values obtained by those coupons might be unrealistic.

Despite the aspects commented above, some Finite Element (FE) analyses to study the shear stress $\left(\tau_{12}\right)$ field of these coupons have been performed. Hence, the results for short coupons were compared to simulations of a standard coupon (Figure 35). Thus, it was possible to observe that near the coupon center there is a region with constant stress field. Therefore those small coupons could be used to study the damage evolution by quasi-static cycles tests.

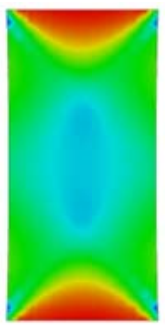

(a)

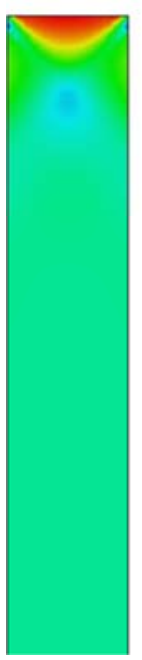

(b)

Figure 35: FE analyses - longitudinal displacement field for $50 \times 25 \mathrm{~mm}$ gage length coupon (a) and $250 \times 25 \mathrm{~mm}$ gage length coupon (b) - coupons with fibers at $\pm 45^{\circ}$ 


\subsubsection{Materials and Methods}

The coupons investigated by Tita (2003) were unidirectional carbon fiber with epoxy resin and, they had similar fiber volume fraction like the filament winding coupons used in this work. The $\pm 45^{\circ}$ coupons dimensions were $250 \times 25 \times 2.7 \mathrm{~mm}$ (ASTM D3518, 2007) and, they have been used to determine the elastic properties as well as strength values. Hence, in order to identify the damage parameters related to the material model, it was necessary to carry out two (2) extra cycle tests.

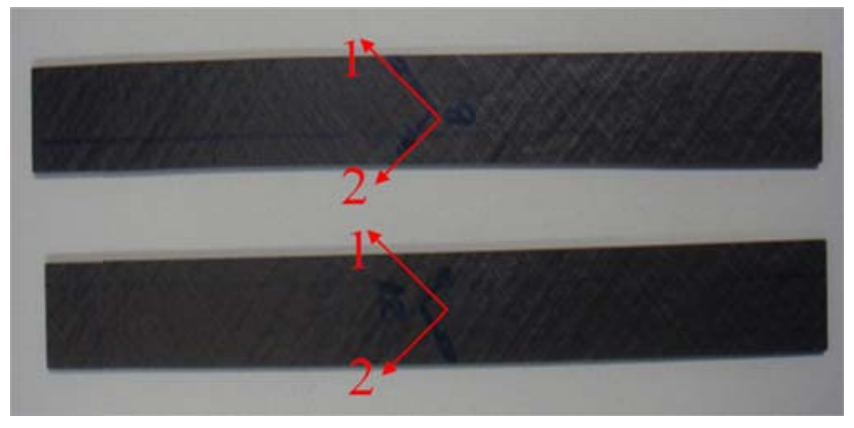

(a)

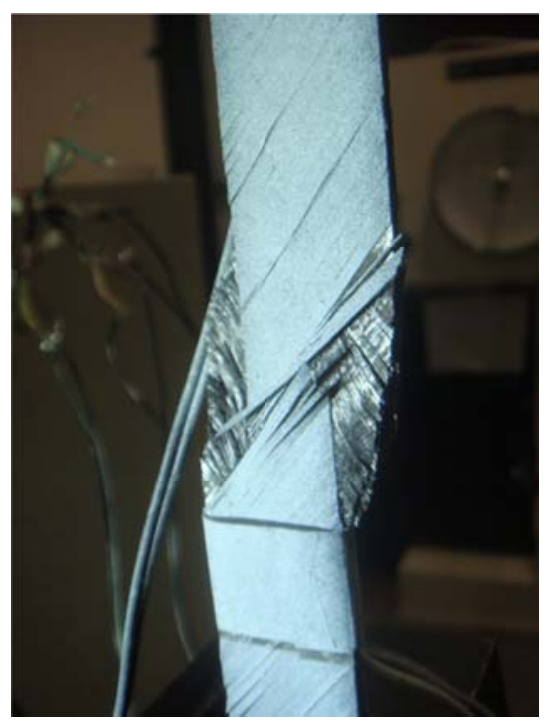

(b)

Figure 36: $\pm 45^{\circ}$ coupons (direction 1 and 2 - fiber orientations) (a); Coupon with failures (b).

\subsubsection{Results and Discussions}

The shear elastic properties as well as the strength values are presented in Table 12.

Table 12: Shear elastic propertie and strength value (Tita V., 2003).

\begin{tabular}{cc}
\hline Elastic propertie and strength value & Values \\
\hline $\mathrm{G}_{12}$ & $5.4 \mathrm{GPa}$ \\
\hline $\mathrm{S}_{12}$ & $53 \mathrm{MPa}$
\end{tabular}

Since only two coupons were available to be tested, the monotonic test has not been performed. In fact, the monotonic tests were carried out by Tita (2003). The in-plane shear 
test results are shown in Figure 37 where it is observed only one curve with coherent response (CDP 45-1). There is also an abrupt interruption on the stress-strain curve due to strain-gage detachment.

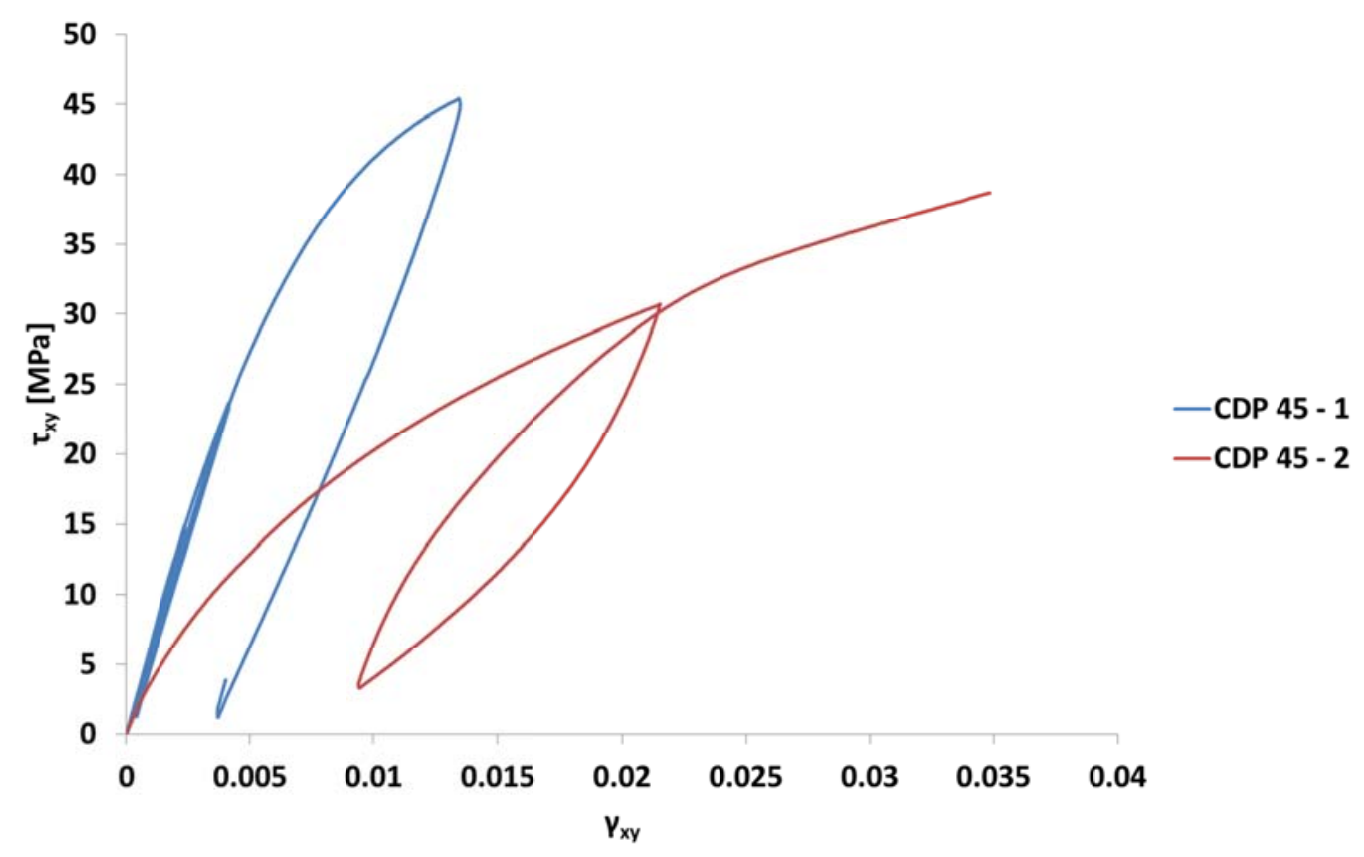

Figure 37: Shear test results.

Once the displacements in the test are rather high and the strain gages data had been lost before the test ending, the force vs. displacement curve can be used to improve the study of damage evolution (Figure 38). 


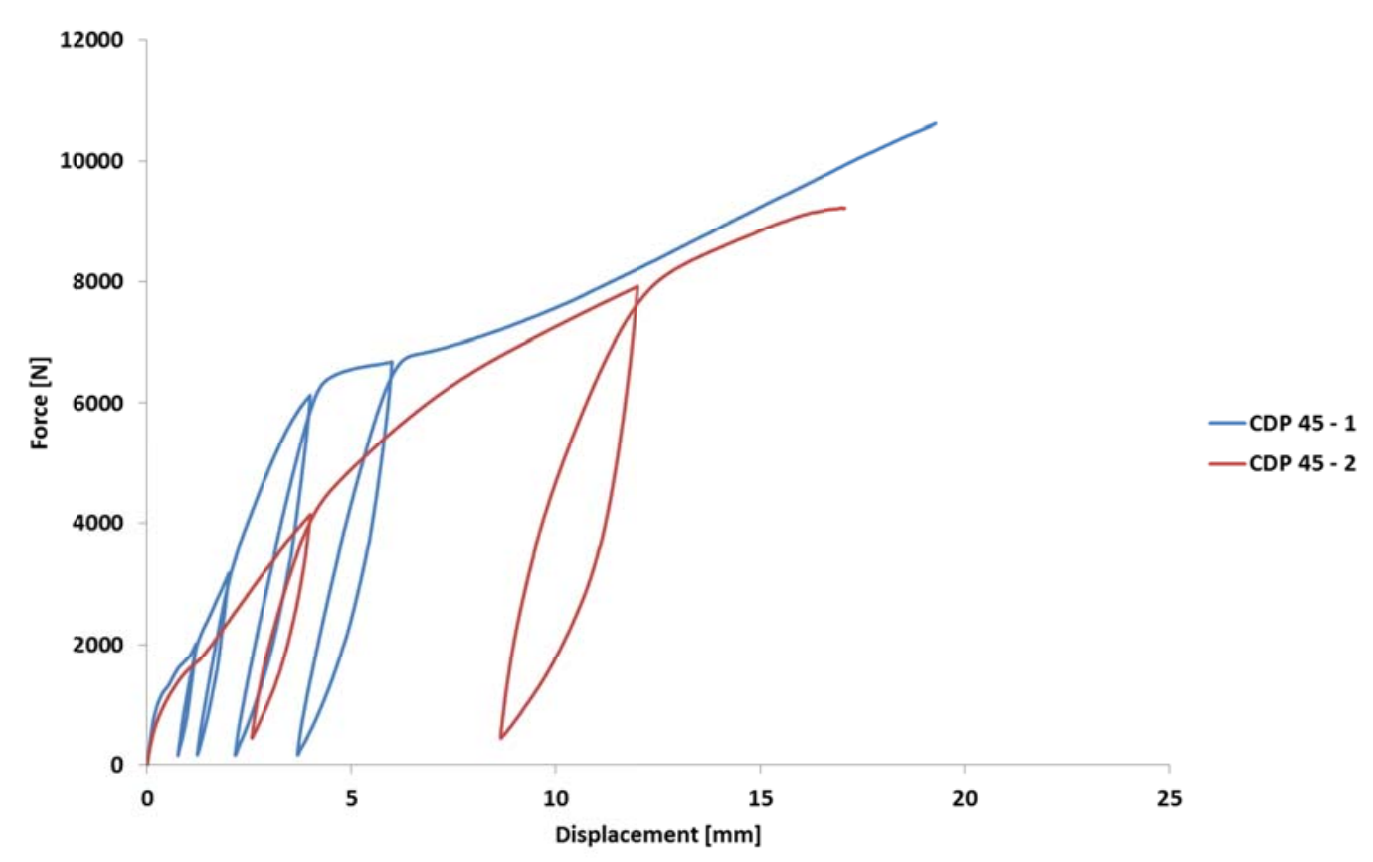

Figure 38: In-plane shear test force vs. displacement curve.

\subsubsection{Shear Tests Conclusions}

Despite the approached problems those results allow evaluating the in-plane shear damage evolution, once the damage measurements consist of a ratio between the initial stiffness and the $\mathrm{i}^{\text {th }}$ cycle stiffness. Besides, the elastic properties and strength values are regarded to be the same as those obtained by Tita (2003) on his PhD thesis because the materials characteristics are quite similar. Moreover, based on the comparison between obtained responses and literature data, the elastic properties and strength values measured for coupon CDP 45-2 have not been considered in the present work. However, the damage measurement was coherent and these results were used.

\subsubsection{BENDING TESTS}

Four point bending tests were performed for off-axis $30^{\circ}$ coupons in order to evaluate the potentialities and limitations of the material model proposal.

Those bending tests followed the guidelines described by ASTM D790 (2008). The coupon dimensions for flexural tests were $25 \mathrm{~mm}$ wide, $180 \mathrm{~mm}$ long and $2.0 \mathrm{~mm}$ thick. The 
external span was equal to $120 \mathrm{~mm}$ and the internal span was equal to $60 \mathrm{~mm}$. Figure 39 shows the schema of four point bending tests, which were carried out until the complete collapse of the coupons.

An important remark is that the off-axis coupons are almost flat plates; so coupons either rotate or translate on the static device supports (inferior cylinders - Figure 39(a)). To ensure the tests repeatability, the coupons have been set in the test device always in the same way, ensuring that the concave side of the plate touches the movable supports (superior cylinders Figure 39(a).

Once the almost flat coupons have a considerable large radius (more than $7000 \mathrm{~mm}$ when the length is equal to $180 \mathrm{~mm}$ ), the displacements in longitudinal direction are small and the effect of friction on the force and displacement results may be neglected.

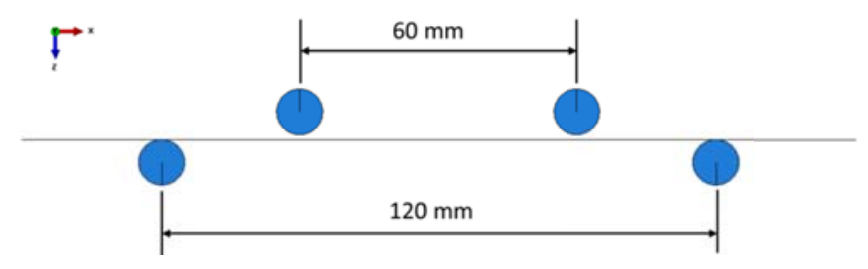

(a)

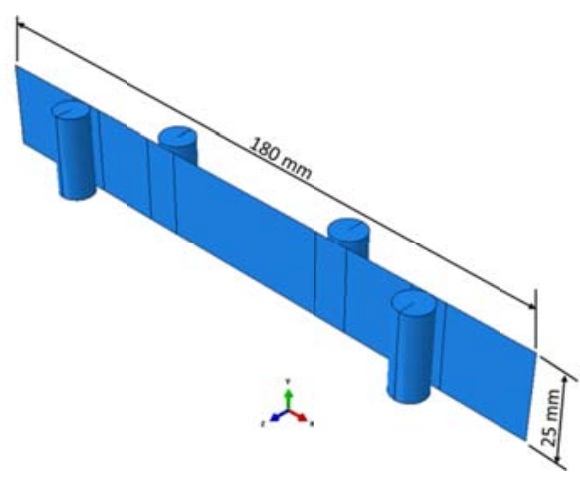

(b)

Figure 39: (a) Four point bending internal and external span dimensions (inferior and superior cylinders). (b) Four point bending schema and test coupon dimensions.

It is important to mention that off-axis coupons have bending-stretching coupling. Hence, in this case, the filament wound composite laminates show a more complex displacement due to the warping phenomenon. This kinematic behavior leads to a more complex stress state, which represents a major challenge to predict the onset of material failure and the damage evolution. Moreover, it is important to highlight that inter-laminar stresses in the off-axis laminates are not as discontinuous as in laminates with different ply orientations. This observation improves the delamination strength for off-axis laminates. Thus, that is why, in this case, only intra-ply damage was evaluated during the progressive failure analysis, which will be detailed in the next chapter. 


\subsubsection{Results and Discussions}

Figure 40 shows the total force vs. displacement results for four point bending tests. The experimental analyses show a good convergence of responses among the coupons. Only two coupons, CDP 30-3 and CDP 30-7, show some damage at high load levels before rupture. For the other coupons, neither significant damage nor delamination has been observed prior to collapse. Since the stresses discontinuities are low between layers with the same fiber orientation, delaminations for off-axis laminates are not so common for laminates with different ply orientation.

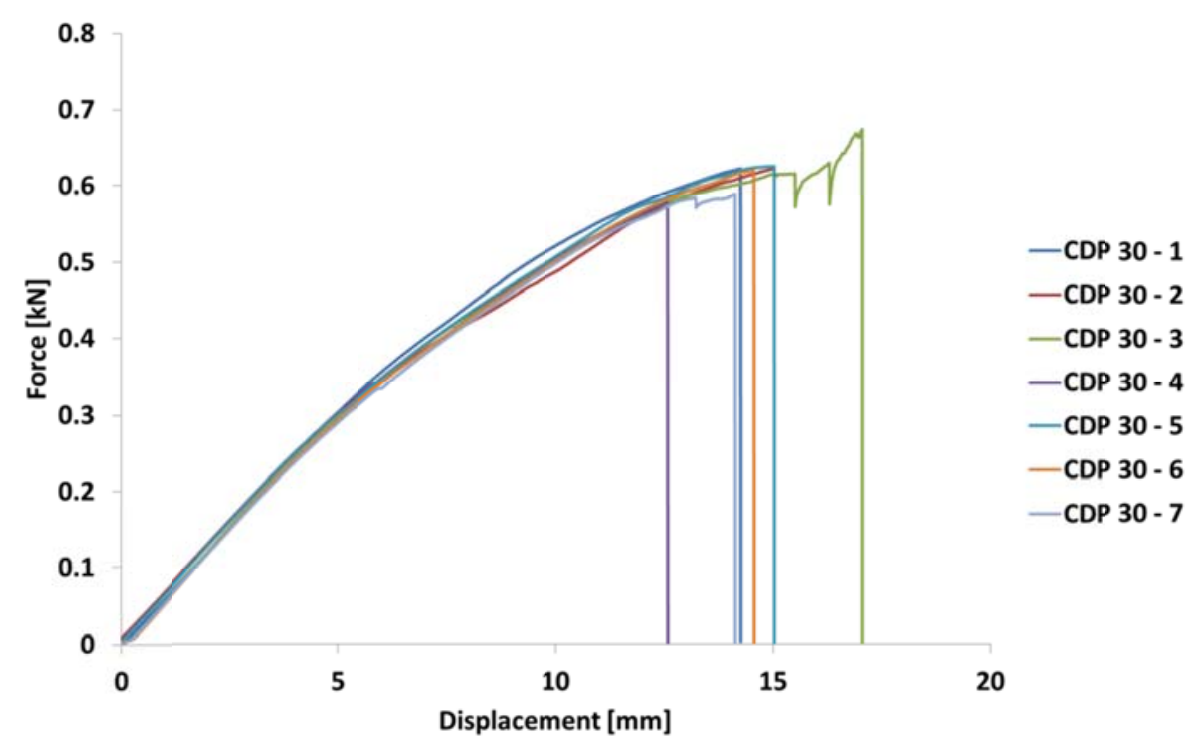

Figure 40: Four point bending test results.

\subsubsection{Bending Tests Conclusions}

The four point bending tests showed a good repetition among the coupons responses, and the results have been coherent with the literature. The curvature effect is small, but it is not possible to use those results in order to investigate only this effect on the response. Likewise, since the stress state in the coupons is more complex, the computational simulation of this test represents a worthy challenge to evaluate the proposed material model. 


\subsection{IMPACT TESTS}

Despite the static behavior of composites being quite well established, it is not possible to say the same for impact loads (Zukas, Nicholas, Swift, Greszczuk, \& Curran, Impact Dynamics, 1982). For example, ASTM D7139 (2007) can be used to guide the impact test for composite flat coupons. However, there is not a standard for impact test on composite cylinders. Moreover, several studies of composite plates under impact have been addressed by some researchers (Donadon, Iannucci, Falzon, Hodgkinson, \& Almeida (2008), Khalili, Soroush, Davar, \& Rahmani (2011), Xiao (2007), Tita, Carvalho, \& Vandepitte (2008)), but only few studies were performed, regarding impact on curved geometry (Kobayashi \& Kawahara (2012), Ballère, Viot, Lataillade, Guillaumat, \& Cloutet (2009), etc).

\subsubsection{INTRODUCTION}

The usage of composite materials in aeronautical industry has increased considerably in the last decades, even in large civil aircrafts as previously commented. Both high stiffness and low weight are the driving factors (Williams, Vaziri, \& Poursartip, 2003). Considering the structural shapes and component functionality, the intrinsic anisotropy of components allows achieving an optimal material performance. This is the reason why pressure vessels are an important application of composite materials. In fact, composite vessels design guidelines were established more than 40 years ago and it has been recommended high safety factors in order to avoid failures, mainly in pressurized vessels (Kobayashi \& Kawahara, 2012).

Among several causes of damage, failures caused by impact loading is a special category. Composite structures are generally more susceptible to impact damage than similar metallic structures. Impact loads may cause internal damage in composite laminates, what is hardly detected by visual inspection, because the damage is very small, e.g. matrix cracking, delamination and/or fiber breakage. However, this internal damage may cause a severe reduction in the structural strength (Abrate S. , 1998) and, many different parameters affect the structural response. Not only the initial kinetic energy of the impactor is an important parameter, but also the mass and the impact velocity. As reported by Abrate S. (1998), small mass at high velocity produces different damage patterns compared to the impact caused by large mass at low velocity, even if both impactors hit the target (composite structures) at equal kinetic energy. Normally, in low velocity impact, the damage starts with matrix cracking, 
which evolves to delaminations in the interface of plies with different orientations. Despite the high strength in fiber direction, out-of-plane stresses caused by impact loads due to either a bird strike or a dropped tool may lead to severe damage. In metallic structures, this kind of damage is easier to be detected differently from what happens to carbon fiber composite structures (Ballère, Viot, Lataillade, Guillaumat, \& Cloutet, 2009).

There are several testing standards (tensile, compression, shear, bending, fatigue, impact, etc) for flat composite coupons. The standards ASTM D7136 for composite and ASTM D5628 for rigid plastics provide the guides to perform the impact test on flat coupons. Several researchers ( (Tita, Carvalho, \& Vandepitte, 2008), (Abrate S. , 2001) (Christoforou \& Yigit, 2009), (Menna, Asprone, Caprino, Lopresto, \& Prota, 2011), (Quaresimin, Ricotta, Martello, \& Mian, 2013), etc) have also investigated impact problems on flat composite plates. However, there are few publications about impact on curved composite structures ( (Minak, Abrate, Ghelli, Panciroli, \& Zucchelli, 2010) (Ballère, Viot, Lataillade, Guillaumat, \& Cloutet, 2009) (Minak, Abrate, Ghelli, Panciroli, \& Zucchelli, 2010)) and, there is no standard for impact tests on curved composite coupons. Because of the absence of standards, each experimental test presented in earlier research work should be considered as a unique analysis, once those research works have shown different test conditions, e.g. different types of coupons fixture.

Part of the present work consists of experimental analysis in transverse impact tests, which is carried out in carbon fiber filament wound cylinders in order to investigate the effect of some parameters on the impact response. Thus, anisotropy, energy level, boundary conditions and cylindrical shape have been evaluated by experimental tests. Three different cylinder lay-ups were manufactured by using the filament winding process previously described, and two energy levels for the impact tests were applied by using a drop tower equipment. The difference of the response among each lay-up and the influence of energy level have been analyzed in details. 


\subsubsection{MATERIALS AND METHODS}

During the impact tests, force and displacement information of the impactor are measured when it interacts with the coupon. Strains are measured in two different points of the cylinders by using bidirectional strain gages.

All the tests have been carried out in the laboratories of the Materials Engineering Department of the Catholic University of Leuven (KU Leuven - Belgium).

\subsubsection{Cylindrical Test Coupons}

Filament winding is a manufacturing process, which can produce high quality composite cylinders. Thus, this process has been used to manufacture circular tubes with three different lay-ups. Figure 41(a) presents the cylindrical coupon dimensions and, Figure 41(b) shows a picture of the coupon after the cure process in a controlled oven.

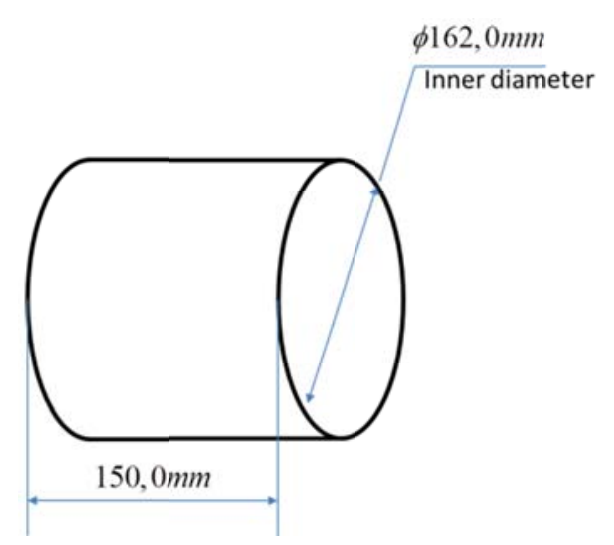

(a)

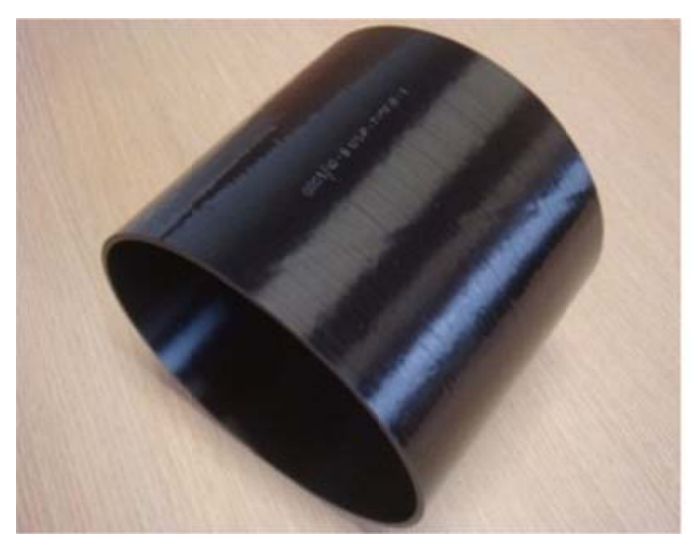

(b)

Figure 41: (a) Coupon dimensions; (b) Cylinder manufactured by filament winding process

Table 13 shows the lay-ups for the composite cylinders, which were identified as being type A $3.49 \mathrm{~mm}$ thick; type B $3.25 \mathrm{~mm}$ thick and type C $3.54 \mathrm{~mm}$ thick. For all types of cylinders, the value of thickness has been calculated based on the average of all nominally identical specimens. Those lay-ups have been used to assess how different levels of anisotropy affect the structural response under impact loads. 
Table 13: Cylinders lay-up and average thickness.

\begin{tabular}{ccc}
\hline Identification & Lay-up & Thickness [mm] \\
\hline Type A & {$[90 / 60 /-60 / 90 / 60 /-60 / 90]_{S}$} & 3.49 \\
\hline Type B & {$[90 / 30 /-30 / 90 / 30 /-30 / 90]_{S}$} & 3.25 \\
\hline Type C & {$[90 / 30 /-30 / 60 /-60 / 30 /-30]_{S}$} & 3.54 \\
\hline
\end{tabular}

Due to the filament winding process, the ply thicknesses depend on the fiber orientation as shown in Table 14.

Table 14: Ply thicknesses for each cylinder type.

\begin{tabular}{|c|c|c|c|c|c|}
\hline \multicolumn{2}{|c|}{ Type A } & \multicolumn{2}{|c|}{ Type B } & \multicolumn{2}{|c|}{ Type C } \\
\hline Orientation & $\begin{array}{c}\text { Thickness } \\
\text { [mm] }\end{array}$ & Orientation & $\begin{array}{c}\text { Thickness } \\
\text { [mm] }\end{array}$ & Orientation & $\begin{array}{c}\text { Thickness } \\
\text { [mm] }\end{array}$ \\
\hline $90^{\circ}(*)$ & 0.29 & $90^{\circ}\left(^{*}\right)$ & 0.28 & $90^{\circ}(*)$ & 0.25 \\
\hline $60^{\circ}$ & 0.29 & $30^{\circ}$ & 0.245 & $30^{\circ}$ & 0.25 \\
\hline$-60^{\circ}$ & 0.29 & $-30^{\circ}$ & 0.245 & $-30^{\circ}$ & 0.25 \\
\hline $90^{\circ}$ & 0.25 & $90^{\circ}$ & 0.24 & $60^{\circ}$ & 0.26 \\
\hline $60^{\circ}$ & 0.25 & $30^{\circ}$ & 0.245 & $-60^{\circ}$ & 0.26 \\
\hline$-60^{\circ}$ & 0.25 & $-30^{\circ}$ & 0.245 & $30^{\circ}$ & 0.25 \\
\hline $90^{\circ}$ & 0.25 & $90^{\circ}$ & 0.205 & $-30^{\circ}$ & 0.25 \\
\hline $90^{\circ}$ & 0.25 & $90^{\circ}$ & 0.205 & $-30^{\circ}$ & 0.25 \\
\hline$-60^{\circ}$ & 0.25 & $-30^{\circ}$ & 0.245 & $30^{\circ}$ & 0.25 \\
\hline $60^{\circ}$ & 0.25 & $30^{\circ}$ & 0.245 & $-60^{\circ}$ & 0.26 \\
\hline $90^{\circ}$ & 0.25 & $90^{\circ}$ & 0.24 & $60^{\circ}$ & 0.26 \\
\hline$-60^{\circ}$ & 0.23 & $-30^{\circ}$ & 0.225 & $-30^{\circ}$ & 0.25 \\
\hline $60^{\circ}$ & 0.23 & $30^{\circ}$ & 0.225 & $30^{\circ}$ & 0.25 \\
\hline $90^{\circ}(* *)$ & 0.17 & $90^{\circ}(* *)$ & 0.16 & $90^{\circ}(* *)$ & 0.21 \\
\hline $\begin{array}{c}\text { Average per } \\
\text { layer }\end{array}$ & 0.249 & $\begin{array}{c}\text { Average per } \\
\text { layer }\end{array}$ & 0.232 & $\begin{array}{c}\text { Average per } \\
\text { layer }\end{array}$ & 0.249 \\
\hline
\end{tabular}

$(*)$ outer layer; $(* *)$ inner layer. 
The variation of the ply thicknesses is due to the manufacturing process. One reason is the application of the same tension in the carbon fiber for all fiber orientations during the filament winding process. Another reason may be the difference of the temperature distribution along the coupon during the cure process, but it has not been further investigated in the present work. Besides, it is important to remember that the manufacturing process is classified.

\subsubsection{Drop Tower Apparatus}

The principle of the impact test via a drop tower apparatus is very simple. The drop test consists of a certain mass, which is dropped from a certain height, hitting the test coupon Force and displacement are measured when the impactor and the coupon are in contact. The displacement of the impactor is measured by using both a Light Emitting Diode (LED) and a light detector (Figure 42). The data recorder only saves the data for the first impact and it does not save any other information, e.g. rebounce effects.

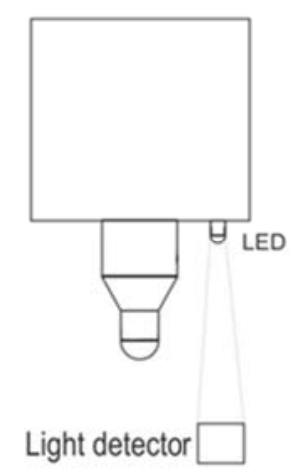

Figure 42: Displacement measurement principle for the impact tests.

The drop tower apparatus (Figure 43(a)) consists of two guiding bars to drive the falling weight during the test. These guiding bars limit the impact height to $1.8 \mathrm{~m}$. The test coupons are set at the base of the tower (Figure 43(a) and (b)). Several types of impactor heads (Figure 43(b)) can be fixed onto the impactor frame for evaluating different types of materials. For harder material, a sharp impactor head is used, but for softer material, a blunter impactor head is recommended. The tests have been performed by using an aluminum round impactor head with a diameter of $16 \mathrm{~mm}$. The round head avoids penetration of the coupon, what would occur if a sharp head were used. A piezoelectric crystal, set between the impactor head and 
the impactor frame, is used as a load cell for impact force acquisition (Figure 43(b)). As commented earlier, the displacement data have been acquired by using a light detector placed at the bottom of the drop tower apparatus, which measures the intensity of a Light Emitting Diode (LED) mounted on the impactor frame. Once the LED is stabilized (constant intensity), the distance measured by the light detector is proportional to $\sim 1 / d^{2}$, where $d$ is the distance between the LED and the light detector. The displacement is set "zero" at the point on the top of the cylinder, when the impactor toughs the specimen. Thus, the distance $d$ is equal the indentation and/or penetration of the impactor through the thickness of the specimens, which are mounted at the base of the drop tower. In this work, the cylindrical coupons have been positioned on a flat surface for impact tests under energy level equal to $8.4 \mathrm{~J}$ (Figure 43(b)) and, in a "V-block" base, for impact tests under energy level equal to $31 \mathrm{~J}$ (Figure 43(c)).

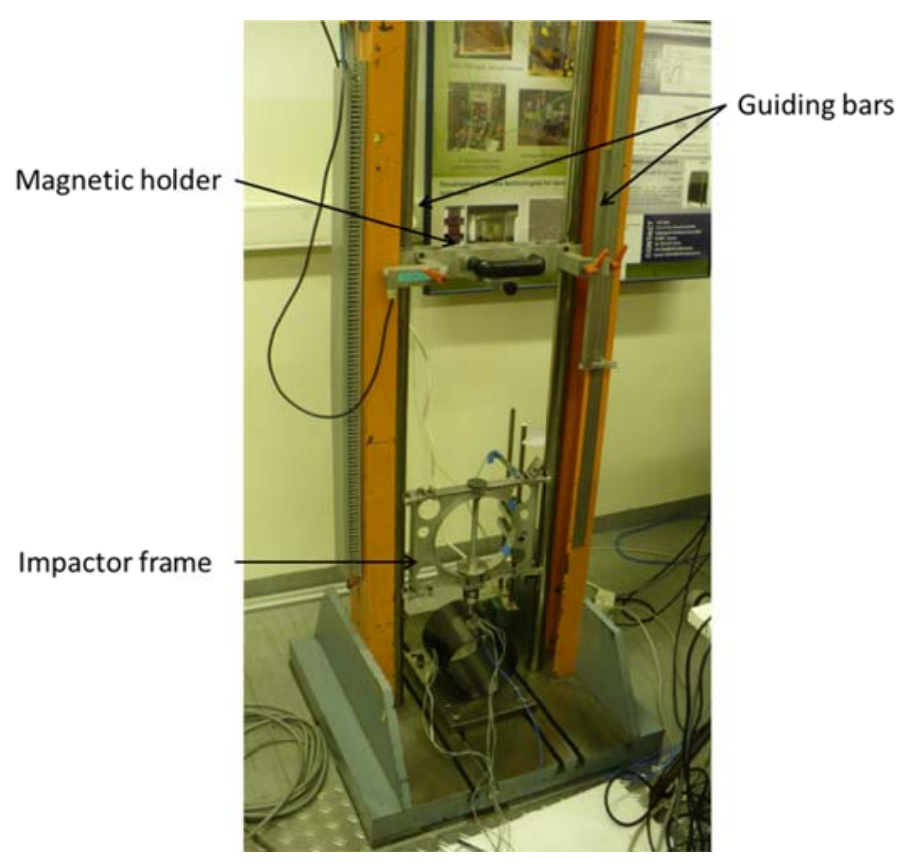

(a)

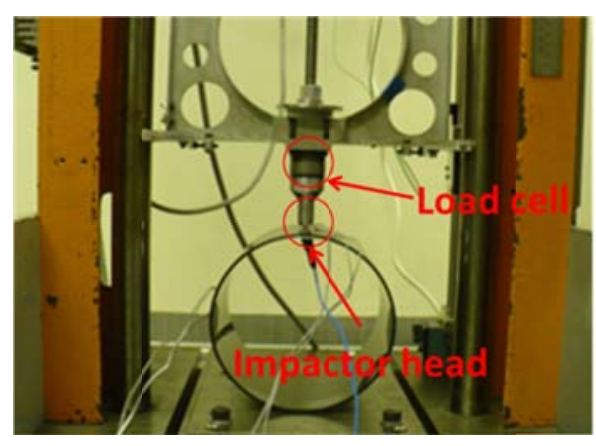

(b)

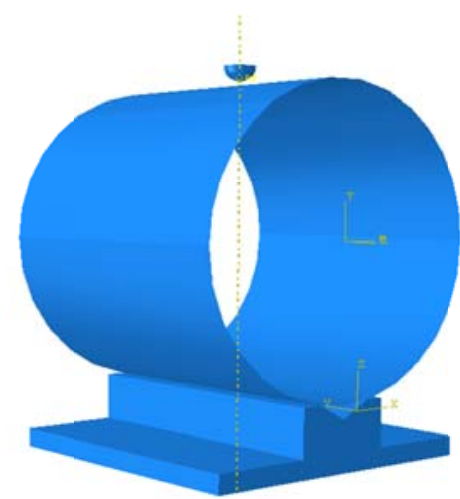

(c)

Figure 43: (a) Drop tower apparatus, (b) Test coupon set at the drop tower base (8.4 J tests); (c)Test schema using V-block at the base ( $31 \mathrm{~J}$ tests) 
The load cell was plugged on a Kistler ${ }^{\mathrm{TM}}$ amplifier (model 5007). This equipment was connected to an acquisition system, which had 11-bit data, with three channels for input and sampling frequency set to $20 \mathrm{kHz}$. The strain measurements were performed by using a HBM amplifier. The software was set to record samples at $20 \mathrm{kHz}$ frequency and the sample size had 64000 points.

As commented above, the impact test equipment provides the information of force, displacement and time when the impactor and coupon interact during the impact event. Also, bidirectional strain gages provide the strains in two different points of the cylinders as shown in Figure 44. The strain gages "1" (Figure 44) are set as close as poissible to the impactor head. The strain gages "2" (Figure 44) are set at a distance from the strain gages " 1 ", which is around $1 / 4$ of the circumferential perimeter.

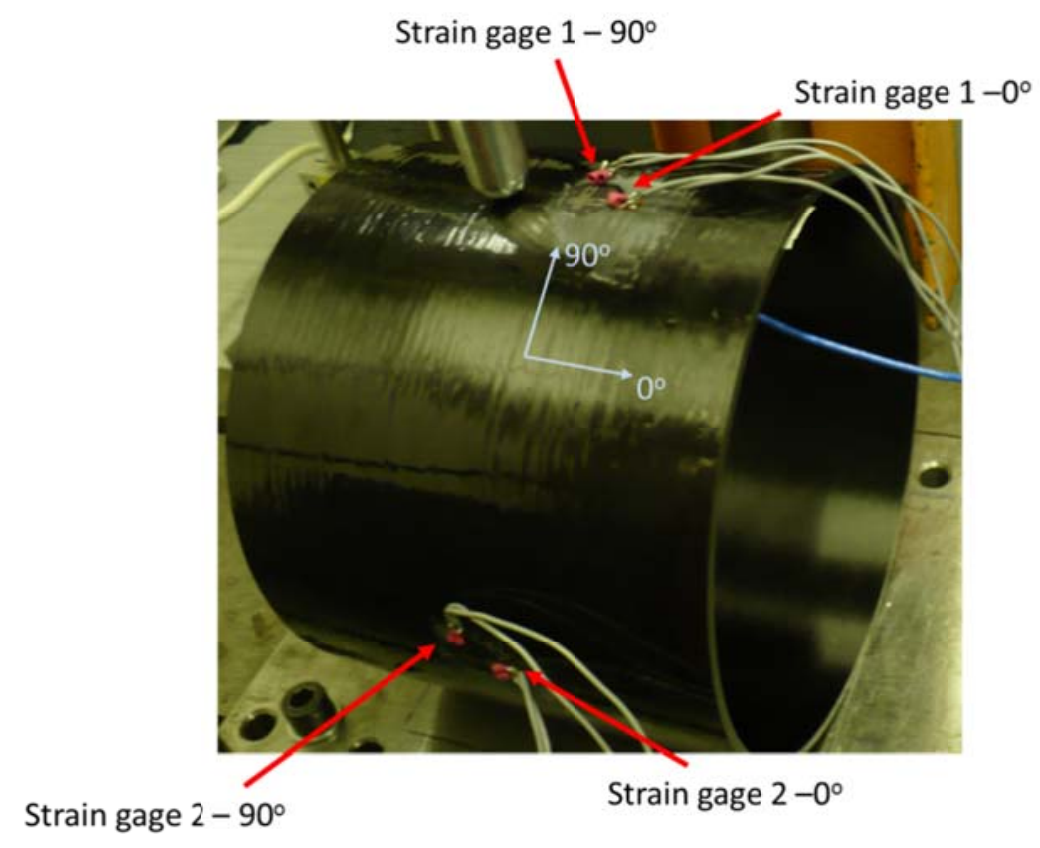

Figure 44: Strain gages position on cylindrical coupons.

Both stain gages are in the middle of the cylinder axial distance in order to measure high values for hoop and axial strains. It is important to mention that the strain gages data were only available for $8.4 \mathrm{~J}$ impact tests. Figure 45 shows the nomenclature used in this Thesis to indicate the strain gage position and the measured strain direction as well. 


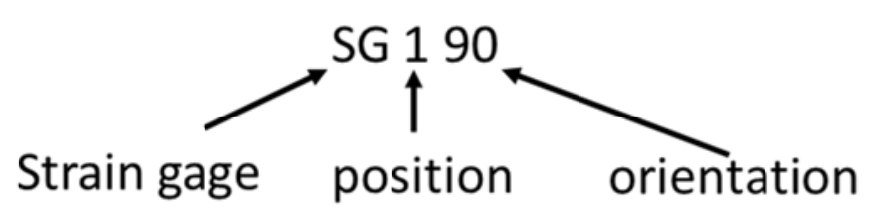

Figure 45: Strain gage nomenclature.

\subsubsection{EXPERIMENTAL ANALYSIS}

The experimental tests have been carried out by using two energy levels ( $8.4 \mathrm{~J}$ and $31 \mathrm{~J}$ ) and three different lay-ups, allowing a critical investigation about the damage process in cylindrical shapes. In addition, it was possible to investigate the influence of the anisotropy and the boundary conditions as well as the impact energy on the impact response. Table 15 shows the number of tests carried out for each cylinder type at both energy levels.

Table 15: Experimental tests repetitions

\begin{tabular}{ccc}
\hline Cylinder type & Number of tests at 8.4 J & Number of tests at 31 J \\
\hline Type A & 4 & 4 \\
\hline Type B & 4 & 4 \\
\hline Type C & 4 & 4 \\
\hline
\end{tabular}

According to some authors, damage initiation is detected in the force vs. time history when a sudden force drop occurs due to stiffness reduction caused by unstable damage growth (Schoeppner \& Abrate, 2000). It is possible to identify the delamination threshold by the first sudden force drop. However, matrix cracking, which is the first type of damage due to impact loading, does not affect the laminate stiffness (Schoeppner \& Abrate, 2000). Thus, it is very complicated to identify this type of damage, only by observing the force signal. Therefore, the experimental analysis developed in this work goes further. In other words, not only the impact test force vs. time history curves have been evaluated, but also the displacement vs. time history and the strain vs. time history as well as the transferred energy graphics have been analyzed in details. 


\subsubsection{Results for Cylinder Type A}

Figure 46 shows the force $v s$. time and displacement $v s$. time for $8.4 \mathrm{~J}$ impact tests on type A cylinders (Table 13). For this cylinder type and energy level, four tests were performed, but only two of them presented good results. Figure 46 shows that either the force or displacement histories are nearly identical for both coupons. The impact duration was around $15 \mathrm{~ms}$. The maximum displacement was around $-6.7 \mathrm{~mm}$ (Table 16).

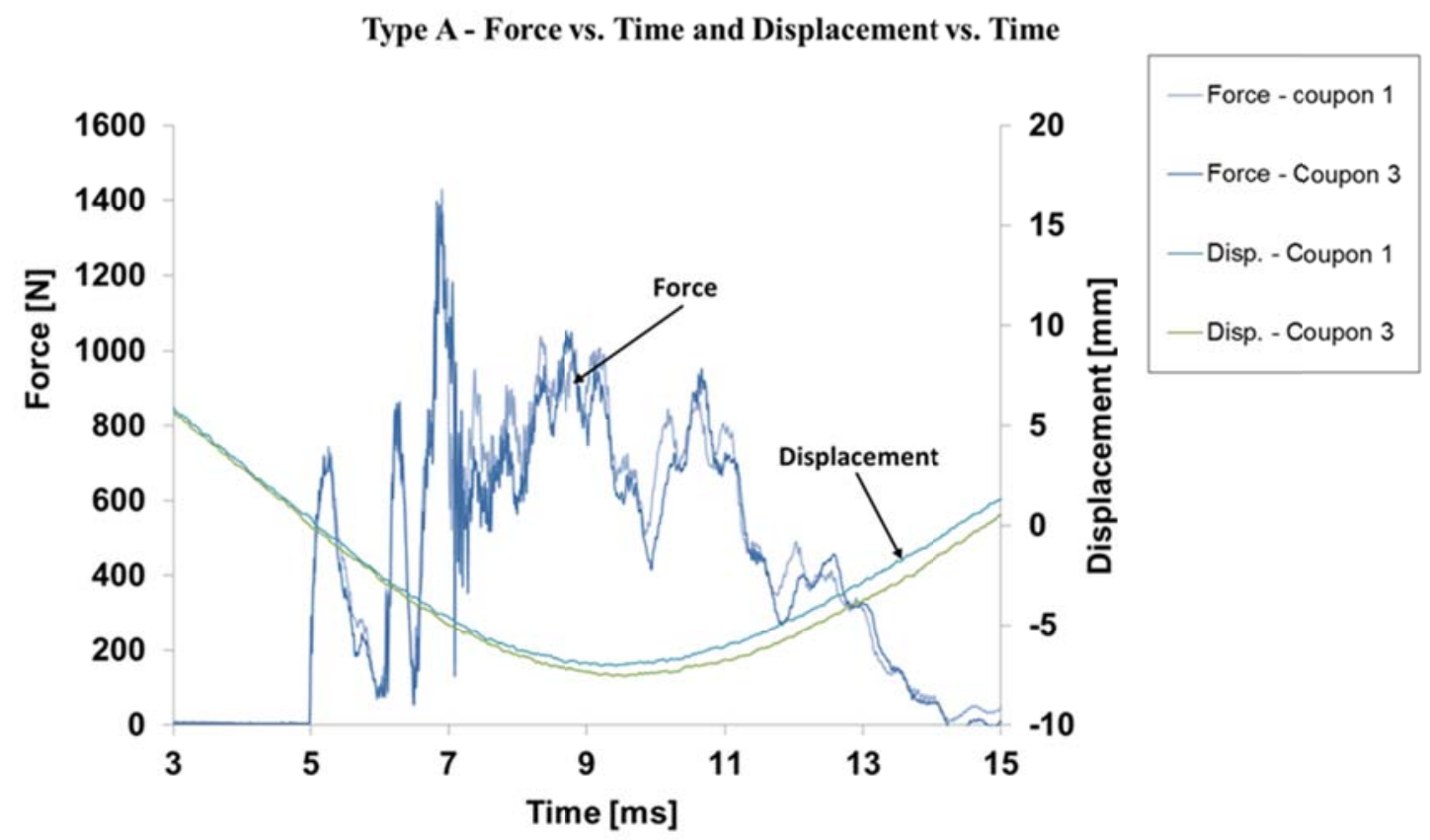

Figure 46: Type A (8.4 J) - force vs. time and displacement vs. time.

The responses for $8.4 \mathrm{~J}$ impact tests on type A cylinders (Figure 46 and Figure 47) show that the force increases quickly close to $5.17 \mathrm{~ms}$ and a sudden force drop occurs. The strain gage SG 190 (Figure 47) in the same period of time (regardless a very small delay of $0.3 \mathrm{~ms}$ in the beginning of the impact event) had a similar behavior. Thus, the strain gage shows a smoother curve and the strain drop was not so pronounced compared to the force drop. Also, the strain value is around minus $0.3 \%$ for the first force trough (at $5.9 \mathrm{~ms}$ ). After that, in Figure 46, the force increases again and a new sudden drop occurs. Few milliseconds after that (at $6.5 \mathrm{~ms}$ ), the force starts to increase again, and the force peak reaches its maximum value (about $1400 \mathrm{~N}$ ). The strain gage did not account neither the peak nor the trough. It is remarkable that the maximum registered force value (around $6.8 \mathrm{~ms}$ ) did not correspond to the maximum displacement point (around $9.5 \mathrm{~ms}$ ) as shown in Figure 46 and neither did the 
maximum strain occur at the maximum force value. In fact, the maximum strain corresponds to the maximum displacement (from $9.3 \mathrm{~ms}$ to $9.9 \mathrm{~ms}$ ) as shown in Figure 46 and Figure 47.

A similar behavior of force peaks and valleys has been registered by Minak et al. (2010). This behavior may indicate the initialization of delamination between several layers. Thus, after damage initiation, the unstable delamination propagation may cause further oscillations in the force vs. time history, as observed by Schoeppner and Abrate (2000) on flat coupons.

\section{Type A - Force vs. Time and Strain vs. Time}

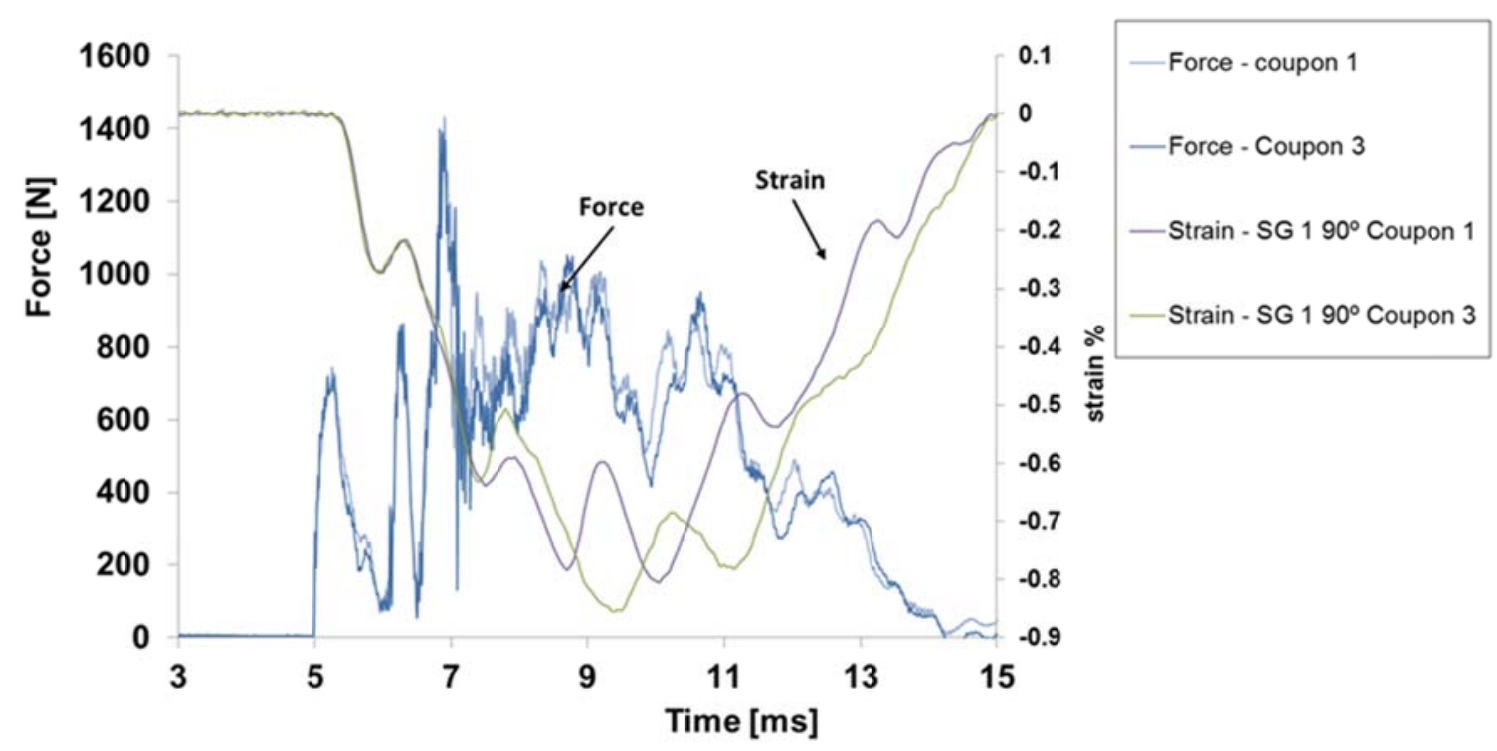

Figure 47: Type A (8.4 J) force vs. time and strain vs. time for SG 190.

Table 16 summarizes the results in force, displacement and strain for type A cylinders under impact energy equal to $8.4 \mathrm{~J}$.

Table 16: Maximum force, displacement and strain for type A cylinders (8.4 J).

Coupon Maximum force peak [N]

Maximum displacement [mm]

(absolute value)
Minimum strain [\%]

\begin{tabular}{cccc}
\hline 1 & 1430.7 & 7.0 & -0.805 \\
\hline 3 & 1396.5 & 7.5 & -0.854 \\
\hline Average & 1413.6 & 7.2 & -0.829 \\
\hline
\end{tabular}

Analyzing the response of all strain gages in Figure 48, a small delay of $1.9 \mathrm{~ms}$ in strain gages " 1 " and " 2 " responses has been observed. This delay corresponds to the time (that) the 
stress wave takes to reach strain gage "2". SG 20 did not measure any strain in the axial direction (Figure 44). For the other coupon, the measured strains behave in similar way.

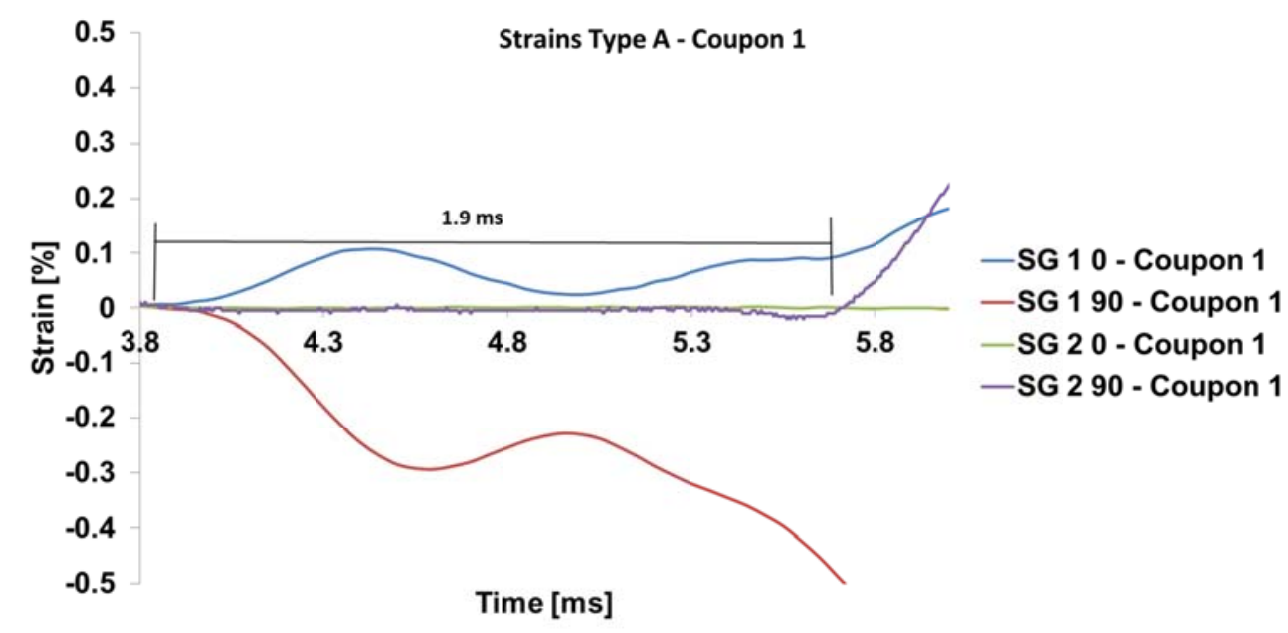

Figure 48: Type A - strains for coupon 2.

Another important parameter, which can be used in the analysis, is the amount of energy transferred from the impactor to the coupon during the impact event, i.e. the impact energy has been converted into elastic energy and dissipated or absorbed energy (depending on the adopted reference). Tita, Carvalho, \& Vandepitte (2008) performed these calculations for composite flat panels. In the present work, the author has used a similar approach, where the energy transferred $\left(E_{t}\right)$ from the impactor to the composite cylinders is shown by eq. (83).

$$
E_{t}=\frac{m v_{0}^{2}}{2}-\frac{m(v(t))^{2}}{2}
$$

where $v(t)$ is the impactor speed at $t(t \geq 0), m$ is the total impact mass (impactor, frame impactor and load cell) and $v_{0}$ is the speed at the impact onset. The impactor speed can be obtained from eq. (84).

$$
v_{t}(t)=v_{0}-\frac{1}{m} \int_{0}^{t} F_{\exp } d t
$$


where $F_{\text {exp }}$ is the measured experimental force using the load sensor. For example, the first part of the time scale in Figure 49, from $4.5 \mathrm{~ms}$ to $9.0 \mathrm{~ms}$, shows how the kinetic energy was transferred from the impactor to the coupon. The kinetic energy transfer occurs in the same slope for all the coupons. The second part of the time scale, from $9.0 \mathrm{~ms}$ to the end, shows the dissipated energy by the cylinder because of the failure mechanisms and the elastic energy, which is represented by the elastic vibrations. Thus, one part of the dissipated energy by the coupon has been related to structural damping. The other part of the dissipated energy has been related to the damage process (Figure 49) as matrix cracking, fiber breakage, delaminations and other failure mechanisms as well. For instance, in the cylinder type A, a small dent mark has been observed in the coupon like an indentation (inelastic deformation).

\section{Type A - Kinetic Energy}

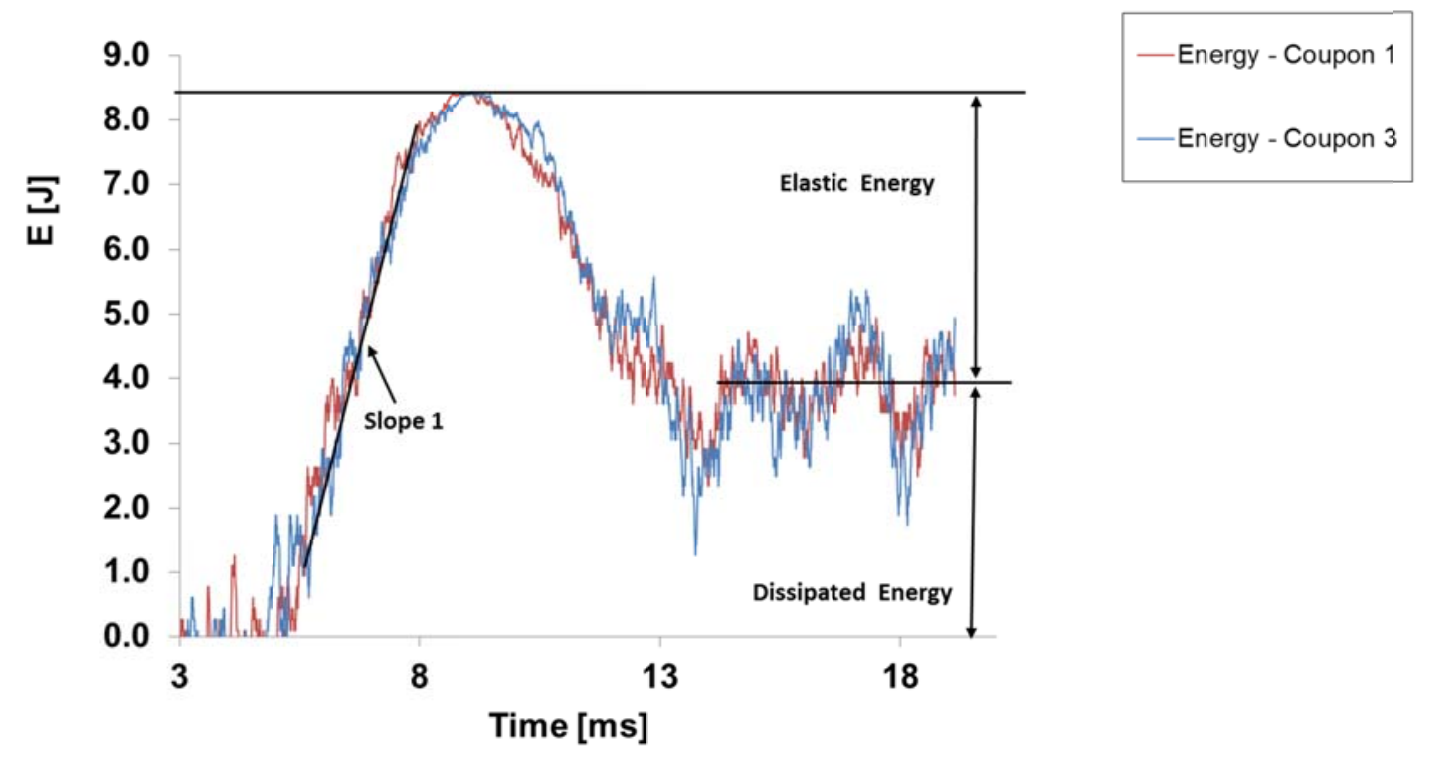

Figure 49: Kinetic energy balance between impactor and type A coupons for $8.4 \mathrm{~J}$ impact test.

Although Figure 49 provides an estimation of the energy that the cylinder can absorb, it is not possible to evaluate neither the value of the energy used to trigger a damage process nor the value dissipated by structural damping. It is further remarkable that damage in the impacted region has not been detected by $\mathrm{C}$-Scan analysis. Thus, it may be concluded that the size of the damage created in the cylinders type A is very small (e.g. matrix micro-cracks), considering $8.4 \mathrm{~J}$ of impact energy level.

Table 17 shows the ratio between the elastic energy (transferred back to the impactor) and dissipated energy (unrecoverable). Considering the lay-up $[90 / 60 /-60 / 90 / 60 /-60 / 90]_{S}$ (type 
A cylinders), impact energy level and impactor type, the elastic energy is around $130 \%$ of the dissipated energy for the coupon 1 . The ratio between elastic energy $\left(E_{e}\right)$ and dissipated energy $\left(E_{d}\right)$ has also been calculated for coupons 2 and 3 . Therefore, the average value for the elastic energy is around $133 \%$ of the dissipated energy (Table 17).

Table 17: Ratio between elastic energy $\left(E_{e}\right)$ and dissipated energy $\left(E_{d}\right)$ - Type A cylinders

\begin{tabular}{cc}
\hline Coupon & $\mathbf{E}_{\mathrm{e}} / \mathbf{E}_{\mathbf{d}}$ \\
\hline 1 & 1.34 \\
\hline 3 & 1.32 \\
\hline Average & 1.33 \\
\hline
\end{tabular}

In order to investigate the influence of the impact energy level, additional mass has been attached to the impactor frame (Figure 43(a)), and the initial impactor height has been increased. This combination of mass and height leads to a potential energy around $31 \mathrm{~J}$ (the exact value is $30.65 \mathrm{~J}$ ).

For type A cylinders under $31 \mathrm{~J}$ impact energy, the force $v s$. time and displacement $v s$. time results are shown in Figure 50. The graphics show that the repetitions produce almost identical results.

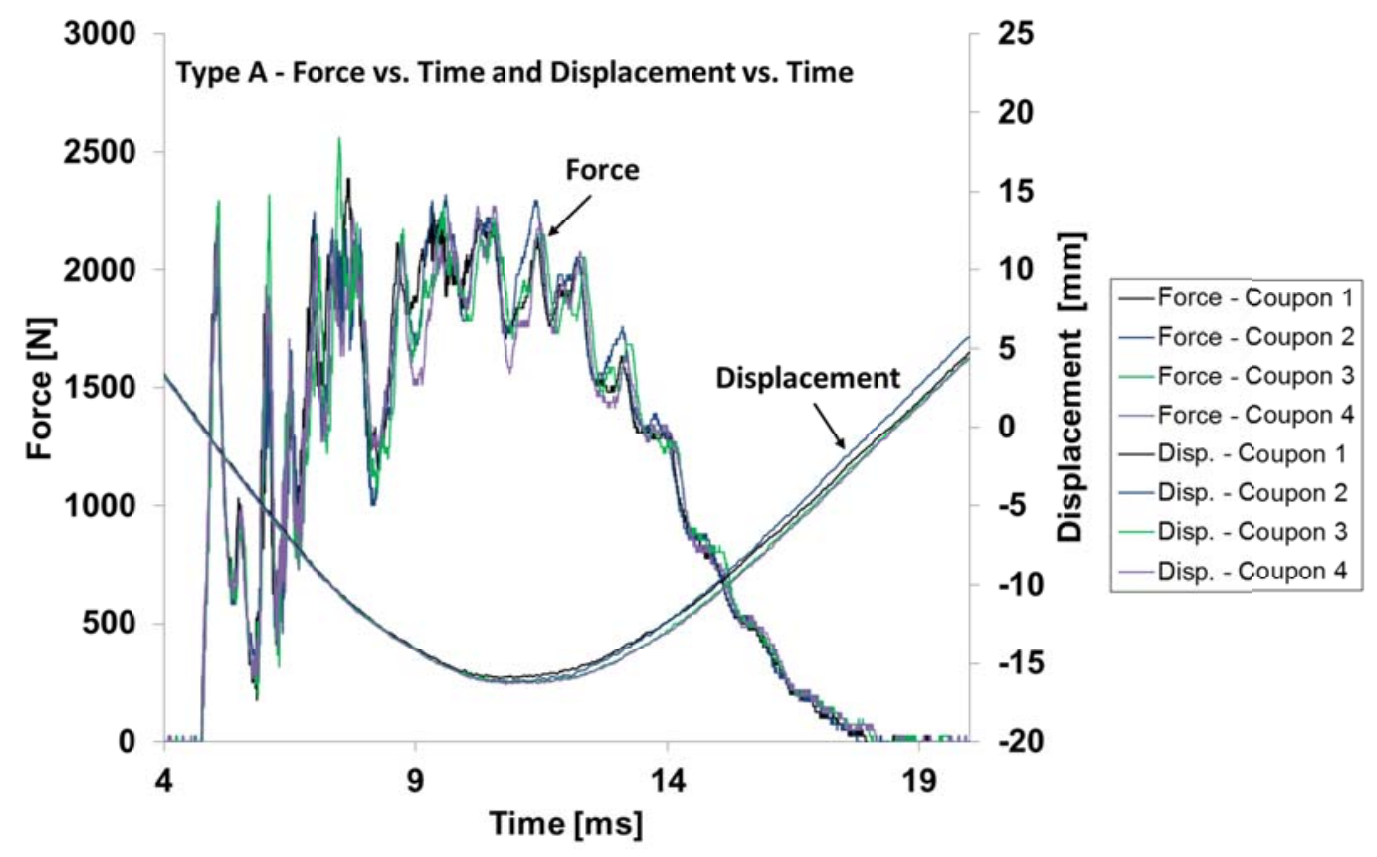

Figure 50: Type A $(31 \mathrm{~J})$ - force vs. time and displacement vs. time . 
$31 \mathrm{~J}$ impact tests on Type A cylinders (Figure 50) show that the force increases quickly close to $5.2 \mathrm{~ms}$ and then a sudden force drop occurs. After that, the force increases again and a new sudden drop occurs. The maximum force peaks (around $2500 \mathrm{~N}$ ) occur from $7 \mathrm{~ms}$ to 7.5 ms for all coupons. Just after this interval, the force drops again. The maximum force peak takes place around $0.0078 \mathrm{~s}$ and the maximum displacement at $11.1 \mathrm{~ms}$ (response delay of 3.3 $\mathrm{ms}$ ). This trend repeats close to $8.7 \mathrm{~ms}$ of the impact event. A similar behavior was registered by Minak et al. (2010). This part of peaks and troughs may be an indication of the initialization of delaminations between several layers. After this period of time (from $4.7 \mathrm{~ms}$ to $8.7 \mathrm{~ms}$ ), the unstable delamination propagation may cause further oscillations in the force vs. time history, as observed by Schoeppner and Abrate (2000) for flat coupons. Figure 50 shows that the maximum force level does not occur in the first peak and that the maximum force value does not occur in the same time of the maximum displacement either. The same behavior has already been observed for $8.4 \mathrm{~J}$ impact tests as previously commented

Figure 51 shows the amount of energy, which is transferred from the impactor to the coupons. When compared to type A coupons tested at $8.4 \mathrm{~J}$, in this case (at $31 \mathrm{~J}$ ), there is a different behavior, because during the initial phase, when the impactor loses part of the kinetic energy, the slope of the curve changes. Thus, firstly, the slope decreases at $23.8 \mathrm{~J}$ (8.19 $\mathrm{ms}$ ) and, then (at $26 \mathrm{~J}$ ), the slope increases again (Figure 51) to a value closer to the first slope. This phenomenon occurs because the impact energy level produces a lot of damage in the coupons. For example, matrix cracking and delaminations have been visible as well as a few fiber failures, which have been detected only near the impact area (Figure 52). The impactor also produces a small dent mark on the coupon (inelastic deformation). 


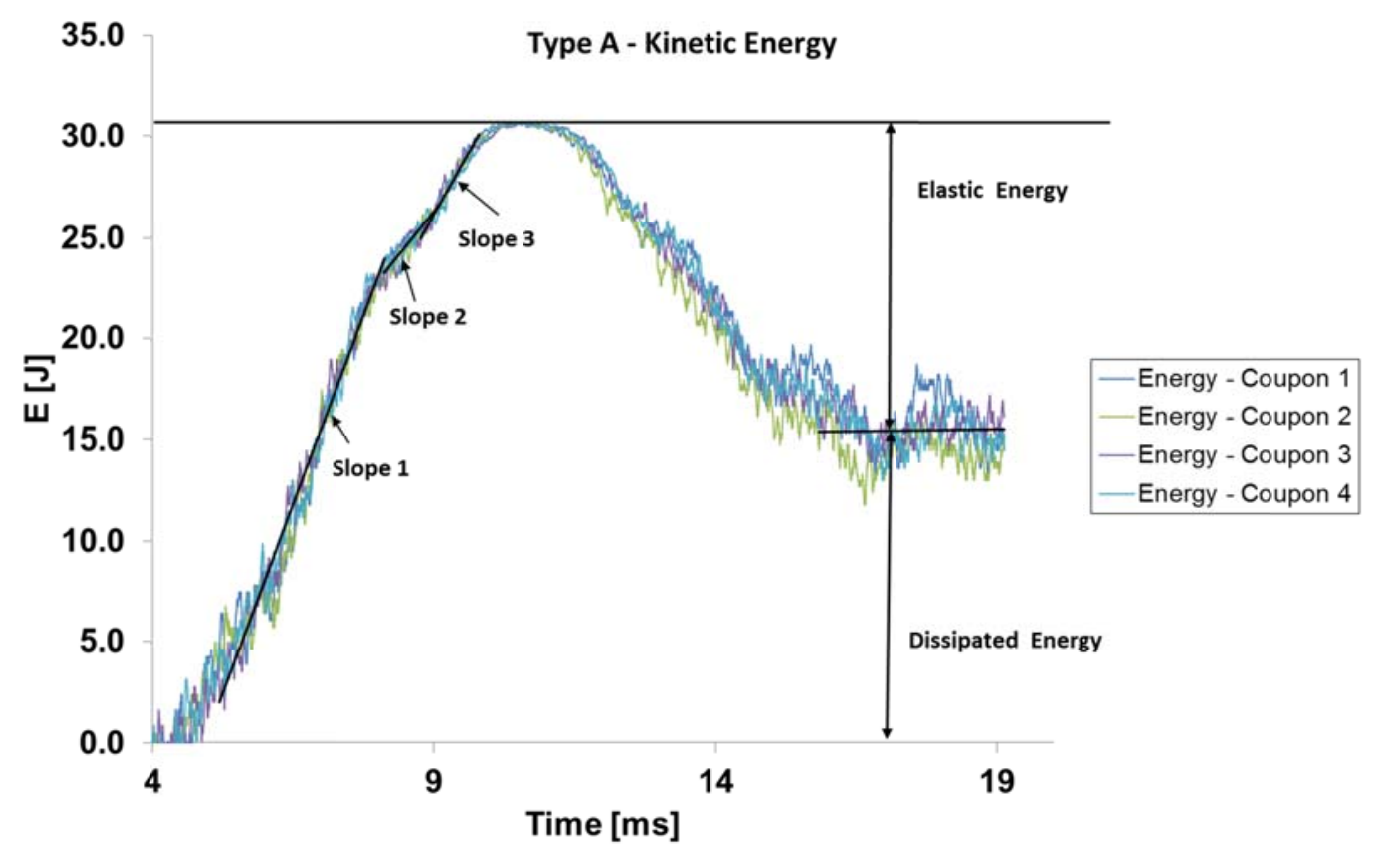

Figure 51: Kinetic energy balance between impactor and type A coupons for $31 \mathrm{~J}$ impact test.

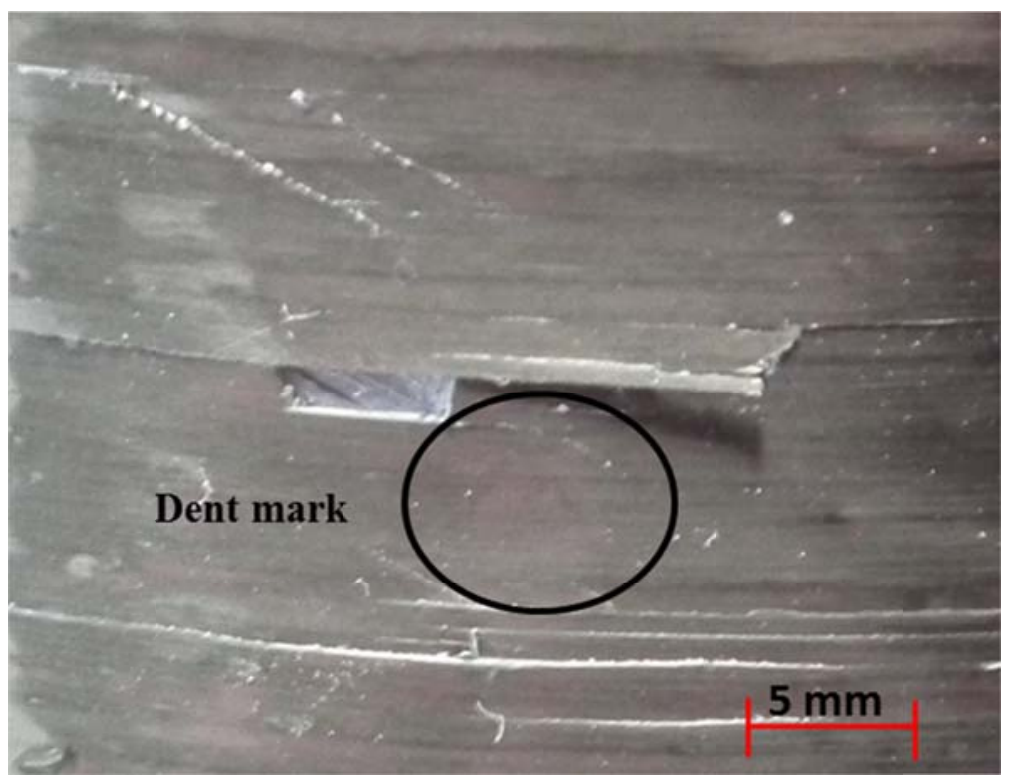

Figure 52: $31 \mathrm{~J}$ impacted area for type A cylinder.

Table 18 shows the maximum peak force value for each coupon as well as the maximum displacement (absolute value). For displacement measurements, the standard deviation is only $1.23 \%$ of the average. For force measurements, the standard deviation is $5.23 \%$ of the average. As observed in the $8.4 \mathrm{~J}$ impact tests, there is no correlation between the maximum measured forces and the maximum displacement for the $31 \mathrm{~J}$ impact tests. 
Table 18: Results summary for type A cylinders (31 J).

\begin{tabular}{ccc}
\hline Coupon & $\begin{array}{c}\text { Maximum force peak } \\
\text { [N] }\end{array}$ & $\begin{array}{c}\text { Maximum displacement [mm] } \\
\text { (absolute value) }\end{array}$ \\
\hline 1 & 2388.9 & 16.0 \\
\hline 2 & 2319.3 & 16.2 \\
\hline 3 & 2563.5 & 16.3 \\
\hline 4 & 2270.5 & 16.3 \\
\hline Average & 2385.6 & 16.2 \\
\hline Standard Deviation & 128.2 & 0.2 \\
\hline
\end{tabular}

The ratio between elastic energy and dissipated energy is around 0.98 (Table 19). Thus, for type A cylinders $(31 \mathrm{~J})$, more energy was dissipated than restored to the impactor. The main sources of unrecoverable energy consist of not only the failure mechanisms discussed earlier, but also the material damping, mainly related to the epoxy matrix.

Table 19: Ratio between elastic energy $\left(E_{e}\right)$ and dissipated energy $\left(E_{d}\right)-$ Type A cylinders $(31 \mathrm{~J})$

\begin{tabular}{cc}
\hline Coupon & $\mathbf{E}_{\mathbf{e}} / \mathbf{E}_{\mathbf{d}}$ \\
\hline 1 & 0.84 \\
\hline 2 & 1.11 \\
\hline 3 & 0.97 \\
\hline 4 & 0.97 \\
\hline Average & 0.98 \\
\hline
\end{tabular}

\subsubsection{Results for Cylinder Type B}

As explained in the previous section, type A cylinders impacted under $8.4 \mathrm{~J}$ did not show any detectable damage close to the impacted area. Thus, this section firstly presents the results for type B cylinders under $8.4 \mathrm{~J}$, also. As verified for type A cylinders, the C-Scan image (Figure 53(a)) shows that there is not any damage for type B. 


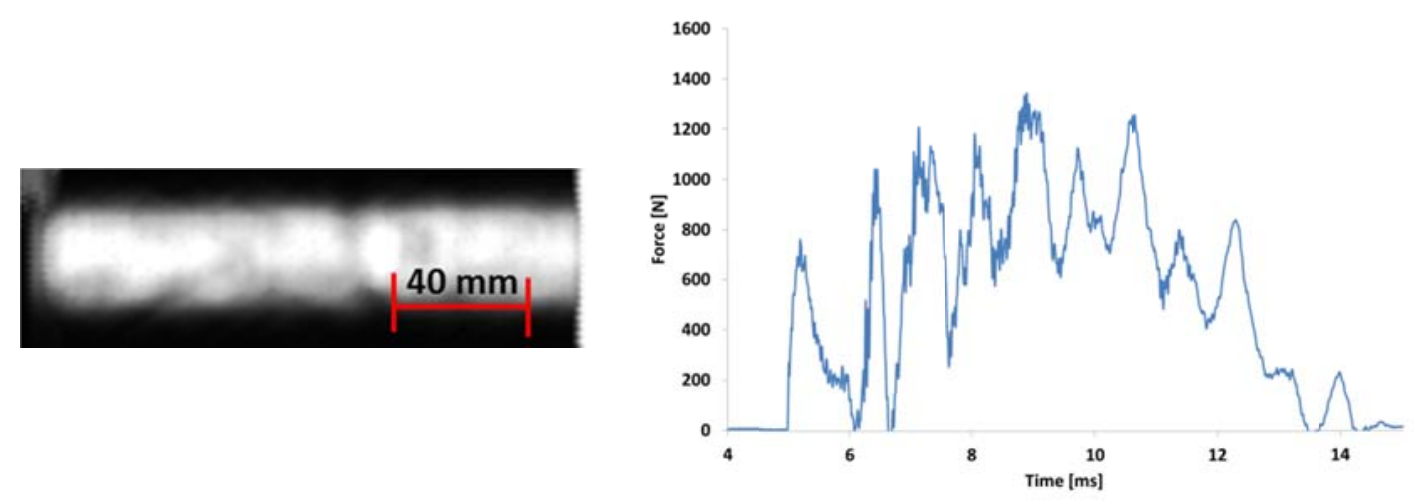

(a)

(b)

Figure 53: Type B cylinders (8.4 J impact energy): (a) C-Scan image; (b) force vs. time (8.4 J).

The force vs. time history for type B cylinders under $8.4 \mathrm{~J}$ impact energy (Figure 53(b)) shows a similar behavior to type A cylinders under $8.4 \mathrm{~J}$ impact tests. This behavior indicates that the peaks and valleys are not only related to delaminations, but also to unstable damage propagation. The effect of boundary conditions and the cylindrical geometry has been pronounced compared to flat plates. Oscillations in the force vs. time may be caused by two sources: 1) the impactor excites the natural modes of the structure (impactor ringing); 2) the coupon reacts with flexural vibrations (ASTM D7139, 2007). Apart of delaminations, the oscillatory behavior may also be explained by the wave propagation across the cylindrical structure and the cylinder modal vibrations as well.

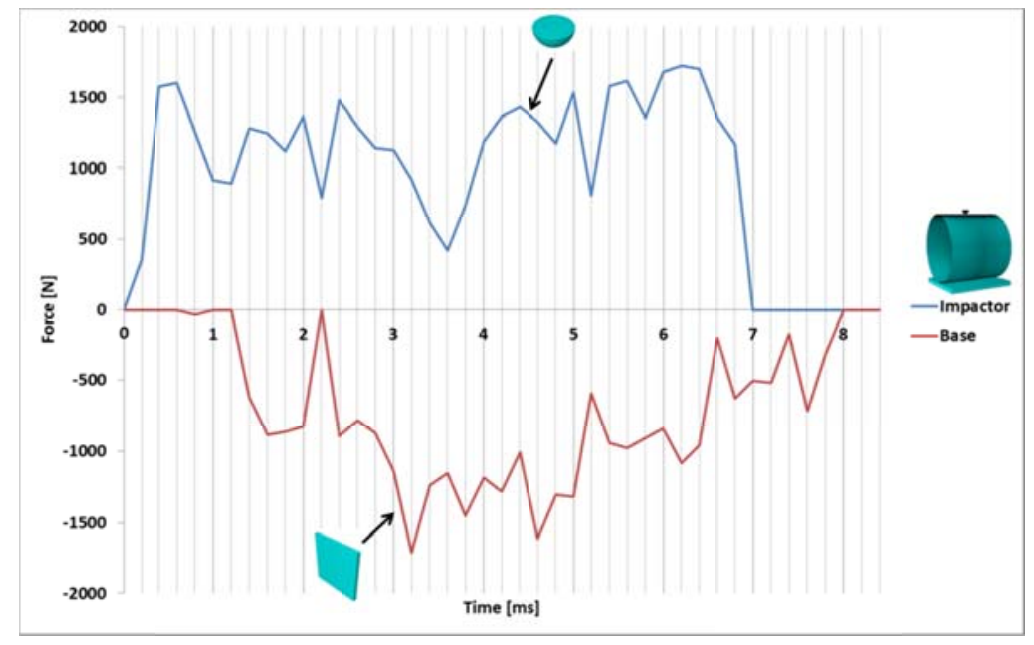

Figure 54: Force vs. time history measured in the impactor and in the base for type B cylinders $-8.4 \mathrm{~J}$ impact energy. 
Figure 54 shows force vs. time history of a finite element simulation for type B cylinders under $8.4 \mathrm{~J}$ impact test. The finite element analysis has been carried out by using ABAQUS $^{\mathrm{TM}} /$ explicit. The composite cylinder was modeled by using 4 node homogeneous reduced integration elements (S4R) and the steel base was modeled by using a 4 node homogenous hexahedron elements. For the impactor, a 3-node discrete rigid element was used. The ABAQUS ${ }^{\mathrm{TM}}$ general contact algorithm regarding hard contact normal behavior was used to model the interactions between the cylinder and the base as well as between the cylinder and the impactor head. Details about the finite element analyses can be found at Ribeiro et al. (2012). It is important to highlight that this simulation did not include neither damage models nor progressive failure analysis. The oscillatory behavior observed in the response of the structure has not been related to any kind of damage. Moreover, the finite element simulations show that there is a response delay provided by the base (reaction force) compared to the input force provided by the impactor (Figure 54). When the impactor just hits the cylinder, the input force increases very fast, but there has been no reaction in the support (base) yet. After $0.6 \mathrm{~ms}$, the reaction force in the base increases, but the force in the impactor decreases. The next force peak of the impactor corresponds to a decrease of the base reaction force. This trend repeats until $3.0 \mathrm{~ms}$ of the impact event, after this time, there are no clear correlations. It indicates that there is a delay of the response between the support reaction and the impactor. The explanation of this effect is the velocity of the wave propagation from the impact point to the base.

Figure 55 shows the force vs. time and displacement vs. time for type B cylinders under 8.4 $\mathrm{J}$ impact. The repetitions have produced nearly identical results. The trend of peaks and troughs is similar to type A cylinder, but for type B cylinders, the maximum peak force occurs closer to the maximum displacement data (around $0.6 \mathrm{~ms}$ ) than type A cylinders (around 2.6 $\mathrm{ms}$ ). 
Type B - Force vs. Time and Displacement vs. Time

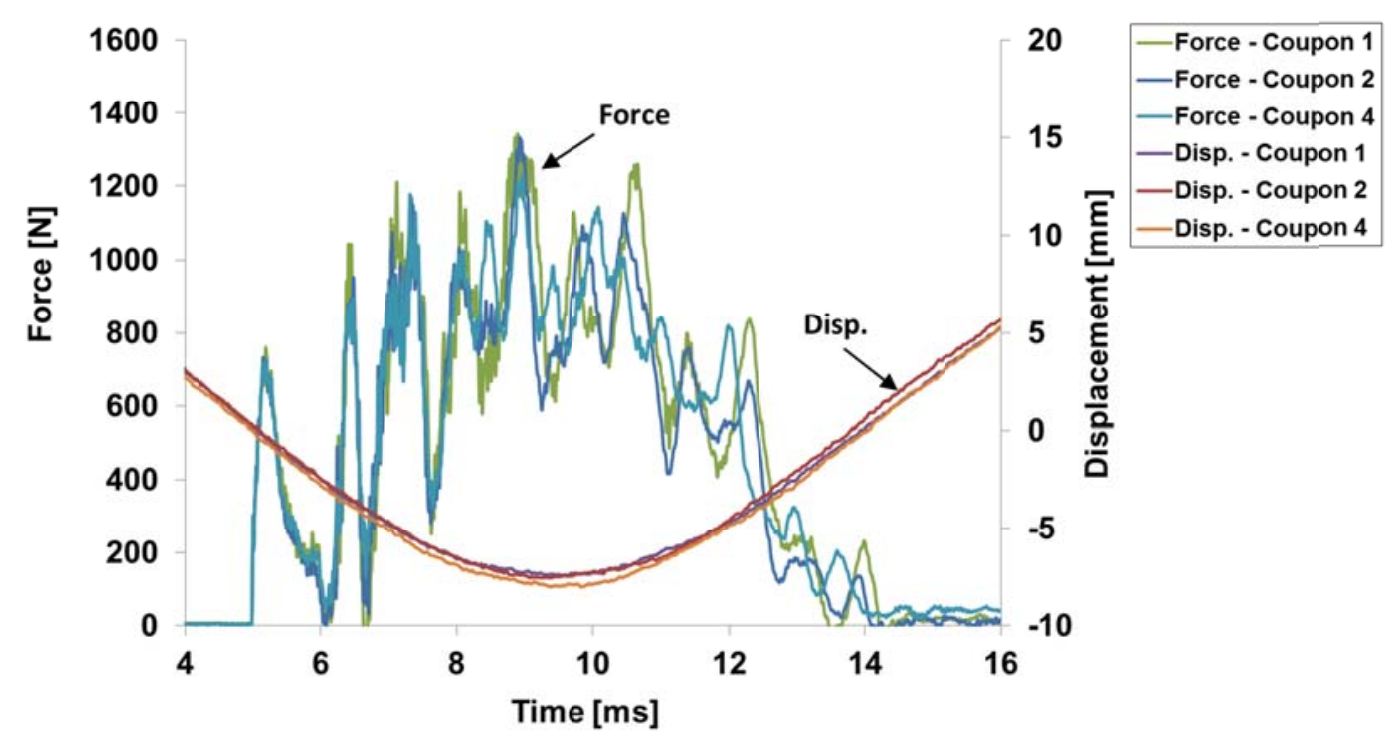

Figure 55: Type B (8.4 J) - force vs. time and displacement vs. time.

The strain vs. time and force vs. time are shown in Figure 56. For cylinder type B under $8.4 \mathrm{~J}$ impact energy level, the maximum force and maximum displacement occur almost in the same point as well as for the minimum strain (response delay around $0.48 \mathrm{~ms}$ ) as shown in Figure 56.

Type B - Force vs. Time and Strain vs. Time

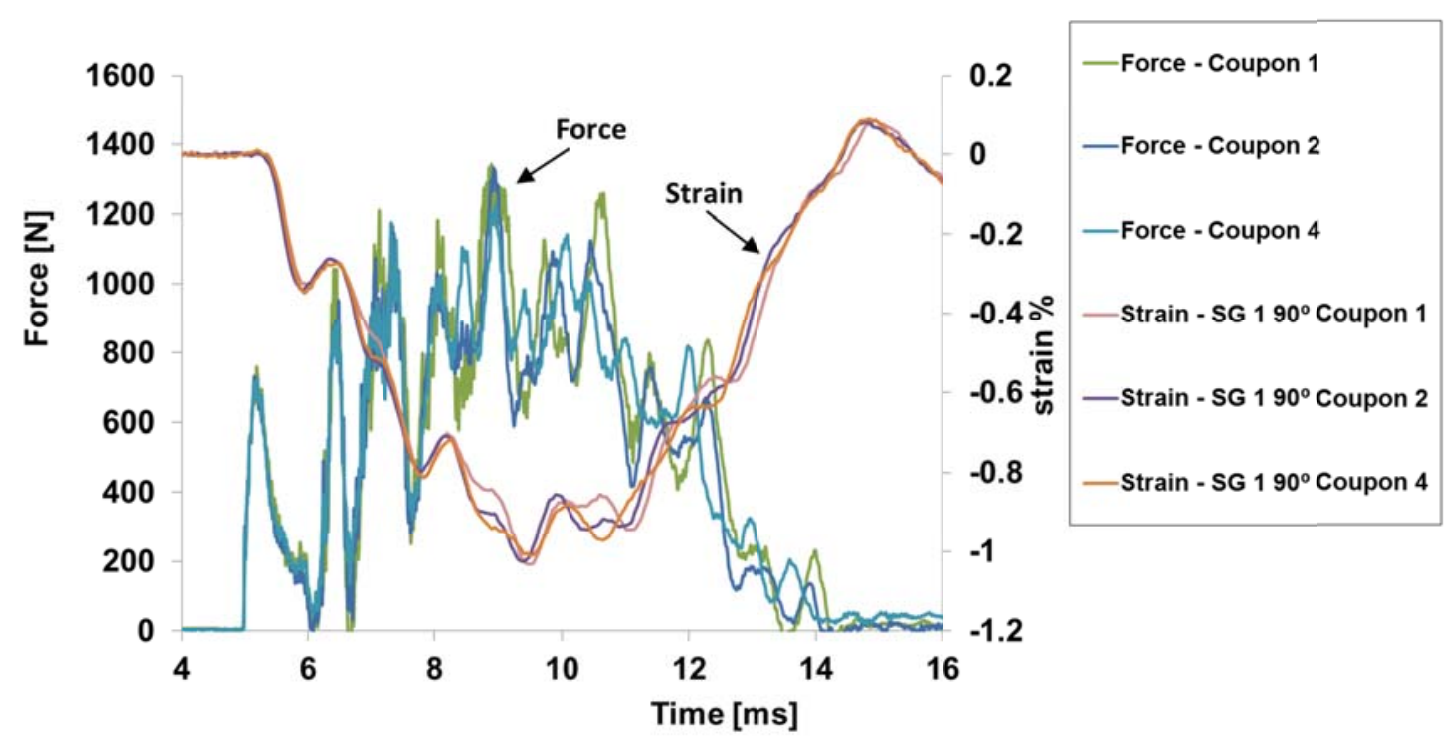

Figure 56: Type B (8.4 J) - force vs. time and strain vs. time. 
Table 20 summarizes the results for force, displacement and strain for type B cylinders under $8.4 \mathrm{~J}$ of impact. The maximum displacement and the minimum strain are larger than the values presented by type A cylinders.

Table 20: Maximum force, displacement and strain for type B cylinders (8.4 J).

\begin{tabular}{cccc}
\hline Coupon & $\begin{array}{c}\text { Maximum force } \\
\text { peak [N] }\end{array}$ & $\begin{array}{c}\text { Maximum } \\
\text { displacement [mm] } \\
\text { (absolute value) }\end{array}$ & Minimum strain [\%] \\
\hline 1 & 1342.8 & 7.4 & -1.031 \\
\hline 2 & 1333.0 & 7.6 & -1.025 \\
\hline 4 & 1228.0 & 8.0 & -1.010 \\
\hline Average & 1301.3 & 7.7 & -1.022 \\
\hline Standard Deviation & 63.6 & 0.3 & 0.018 \\
\hline
\end{tabular}

As observed for type A, the average response delay between strain gages " 1 " and "2" for type $\mathrm{B}(8.4 \mathrm{~J})$ is around $1.5 \mathrm{~ms}$ (Figure 57 ). This value is not so different from that found in type A cylinders $(1.3 \mathrm{~ms})$. Table 21 shows the response delay between strain gages " 1 " and " 2 " for type B cylinders under $8.4 \mathrm{~J}$ impact test. In this case, it has not been possible to measure the delay for coupon 3 . The average delay between the response of strain gages " 1 " and " 2 " for type B is around $1.5 \mathrm{~ms}$. Thus, the response delay for type B cylinders (under 8.4 $\mathrm{J}$ impact test) is $20 \%$ higher than type A cylinders (under $8.4 \mathrm{~J}$ impact test), which indicates the influence of the cylinder lay-up in the stress wave velocity. Since the stress wave has been faster in fiber direction, it is concluded that cylinder type A shows the stress wave velocity faster than that in the other cylinders because its lay-up has fibers at $90^{\circ}$ and $60^{\circ}$. 


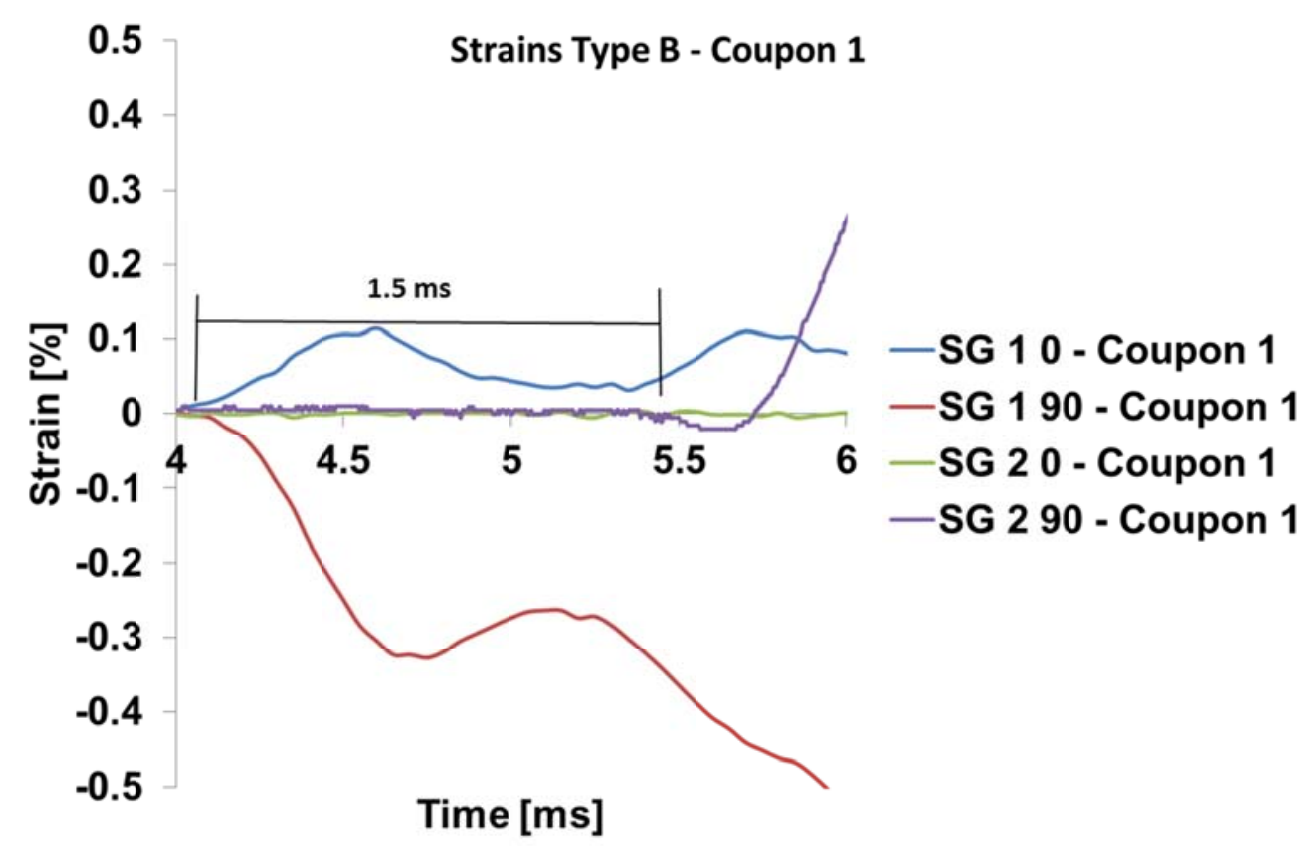

Figure 57: Type B - strains for coupon 1.

Table 21: Delay between strain gage 1 and 2 - Type B cylinders (8.4 J)

\begin{tabular}{cc}
\hline Coupon & Delay [ms] \\
\hline 1 & 1.5 \\
\hline 2 & 1.6 \\
\hline 4 & 1.5 \\
\hline Average & 1.5 \\
\hline
\end{tabular}

Observing Figure 58, the impactor transfers its kinetic energy to the coupon in a single slope curve as observed for type A coupons. The differences between type A and type B cylinders showed very low dissipated energy (Table 22). 


\section{Type B - Kinetic Energy}

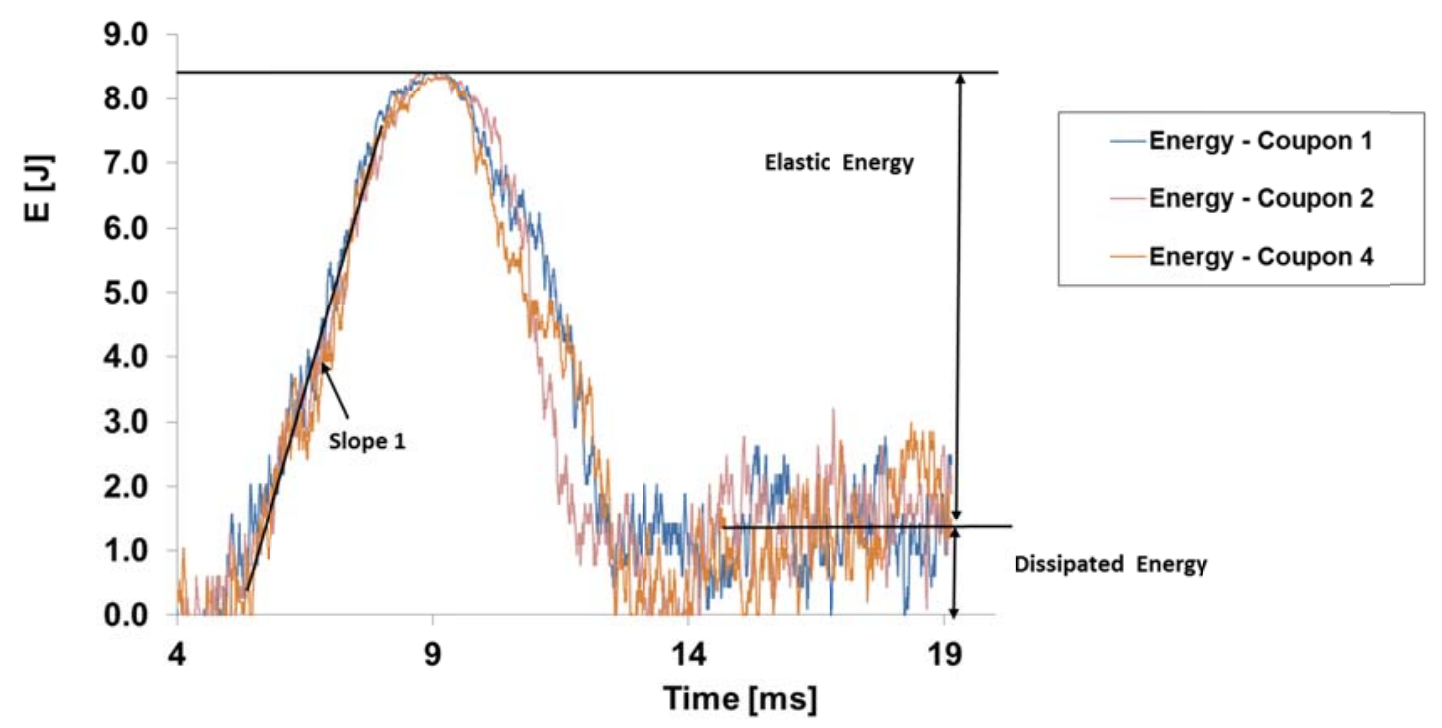

Figure 58: Kinetic energy balance between impactor and type B coupons for $8.4 \mathrm{~J}$ impact test.

The elastic energy is around 7 (seven) times the dissipated energy (Table 22) for type B cylinders. Almost all energy is restored to the impactor and the impact does not cause any significant damage, what confirms the results obtained by C-scan analysis (Figure 53 (a)).

Table 22: Ratio between elastic energy $\left(E_{e}\right)$ and dissipated energy $\left(E_{d}\right)$ - Type B cylinders $(8.4 \mathrm{~J})$

\begin{tabular}{cc}
\hline Coupon & $\mathbf{E}_{\mathrm{e}} / \mathbf{E}_{\mathbf{d}}$ \\
\hline 1 & 6.64 \\
\hline 2 & 6.01 \\
\hline 3 & - \\
\hline 4 & 8.27 \\
\hline Average & 6.98 \\
\hline
\end{tabular}

For type B cylinders, the results of $31 \mathrm{~J}$ impact tests (force vs. time and displacement vs. time) are shown in Figure 59. All experimental data show a good repeatability of the tests. At the same high level of impact energy, type A cylinders and type B cylinders show a similar pattern of peaks and valleys. However, the force peak intensity is higher for cylinder type B, due to the lay-up and the thickness differences of each layer. Delaminations, matrix damage and indentation marks (inelastic deformation) have also been detected for type B cylinders under $31 \mathrm{~J}$ impact tests. 


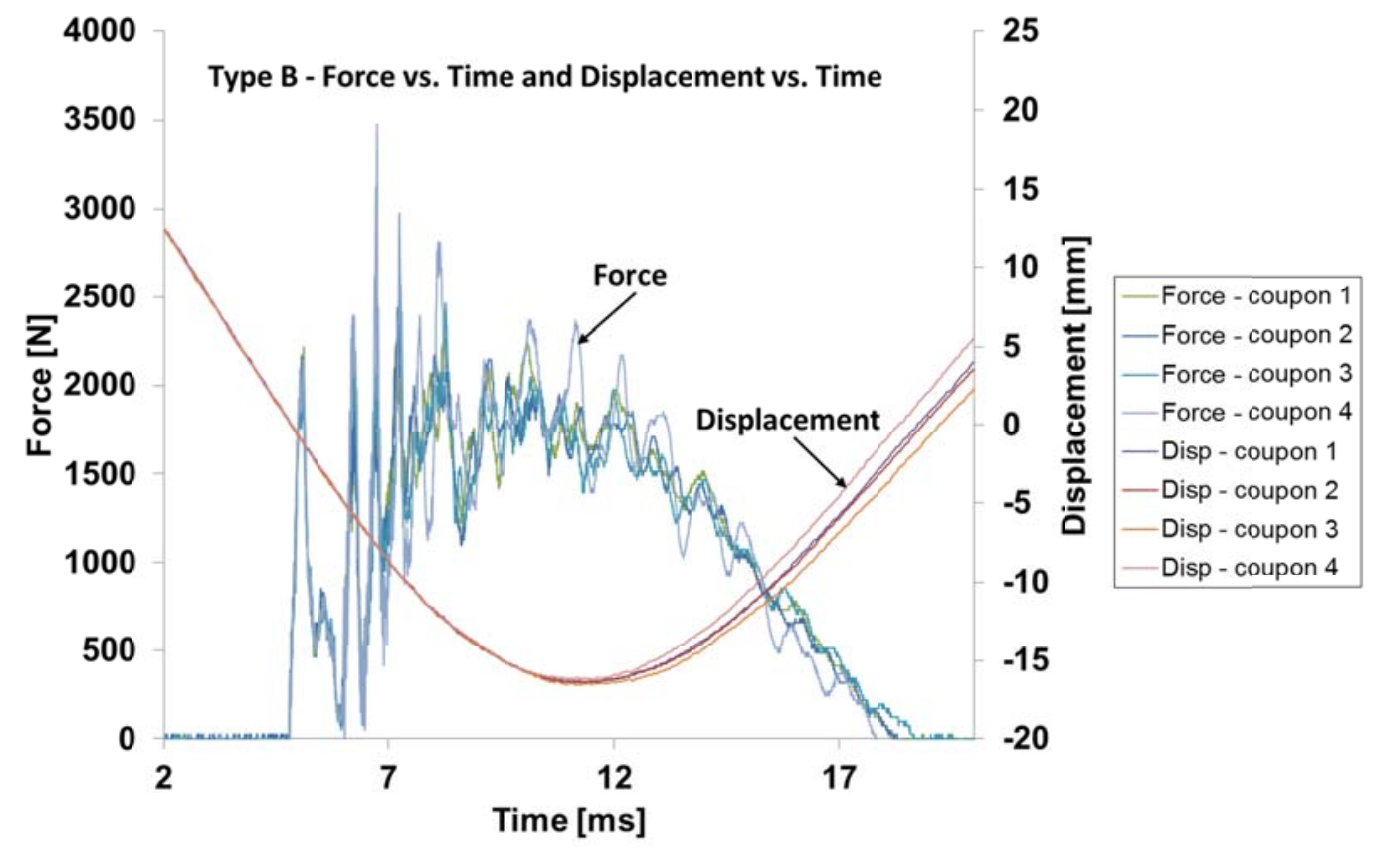

Figure 59: Type B (31 J) - force vs. time and displacement vs. time.

Figure 59 shows that the maximum force peak value occurs around $6.7 \mathrm{~ms}$ and the maximum displacement occurs at $11.2 \mathrm{~ms}$, which represents a response delay of $4.5 \mathrm{~ms}$. Table 23 shows that the standard deviation for the displacement measurements is small. However, from $12.7 \mathrm{~ms}$, it is observed that the displacement measurements start to diverge. For the force values, the standard deviation is $19.1 \%$ of the average value. Thus, there is a considerable dispersion in the maximum peak value measurement. However, regarding the force history for all coupons, they are rather close. Again, there is no correlation between the maximum measured force and displacement.

Table 23: Results summary for type B cylinders (31 J).

\begin{tabular}{ccc}
\hline Coupon & $\begin{array}{c}\text { Maximum force } \\
\text { peak [N] }\end{array}$ & $\begin{array}{c}\text { Maximum displacement [mm] } \\
\text { (absolute value) }\end{array}$ \\
\hline 1 & 2319.3 & 16.4 \\
\hline 2 & 2441.4 & 16.4 \\
\hline 3 & 2612.3 & 16.6 \\
\hline 4 & 3466.8 & 16.2 \\
\hline Average & 2710.0 & 16.4 \\
\hline Standard Deviation & 518.7 & 0.1 \\
\hline
\end{tabular}


Considering the energy balance between the impactor and the coupons for type B cylinders under $31 \mathrm{~J}$ impact tests, the impactor transfers its kinetic energy to the coupons in a bilinear way as shown in Figure 60. This behavior is different compared to type A cylinders $(31 \mathrm{~J})$.

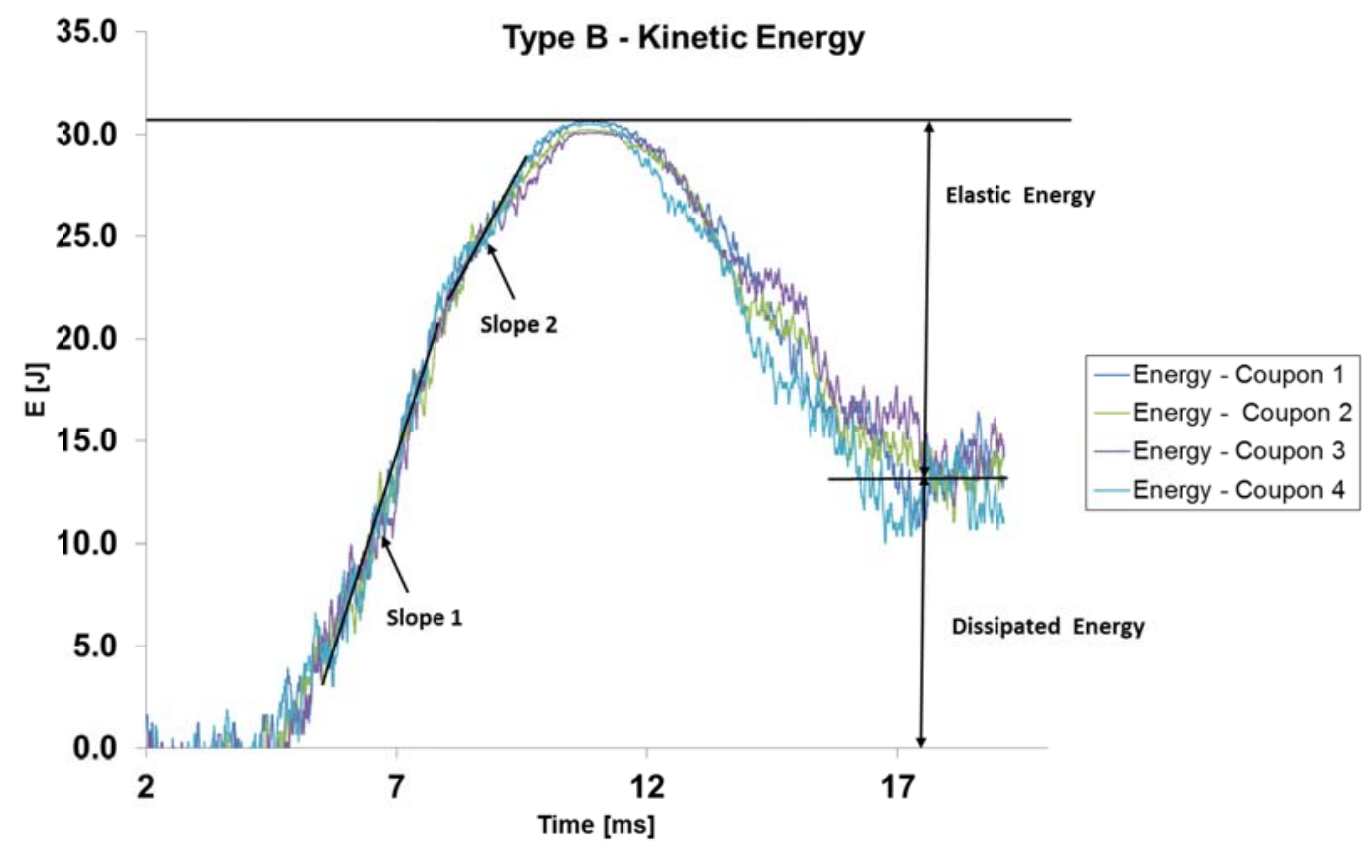

Figure 60: Kinetic energy balance between impactor and type B coupons for $31 \mathrm{~J}$ impact test.

Regarding the restored energy, cylinder configuration B dissipates $77 \%$ (on average) of the elastic energy

Table 24: Ratio between elastic energy (Ee) and dissipated energy (Ed) - Type B cylinders (31 J)

\begin{tabular}{cc}
\hline Coupon & Ee/Ed \\
\hline 1 & 1.30 \\
\hline 2 & 1.33 \\
\hline 3 & 1.15 \\
\hline 4 & 1.44 \\
\hline Average & 1.30 \\
\hline
\end{tabular}

Delaminations have been observed among several plies (Figure 61) in some coupons. As expected, the delaminations occur between plies with different orientations (Abrate S. , 1998) and the delaminations extend from the impacted area to the free edges of the cylinder. It can be observed that $25 \%$ less energy has been dissipated by type B rather than type A cylinders. 
Table 24: Ratio between elastic energy $\left(E_{e}\right)$ and dissipated energy $\left(E_{d}\right)-$ Type B cylinders $(31 \mathrm{~J})$

\begin{tabular}{cc}
\hline Coupon & $\mathbf{E}_{\mathbf{e}} / \mathbf{E}_{\mathbf{d}}$ \\
\hline 1 & 1.30 \\
\hline 2 & 1.33 \\
\hline 3 & 1.15 \\
\hline 4 & 1.44 \\
\hline Average & 1.30 \\
\hline
\end{tabular}

In addition, more energy has been restored than dissipated due to the impact conditions.

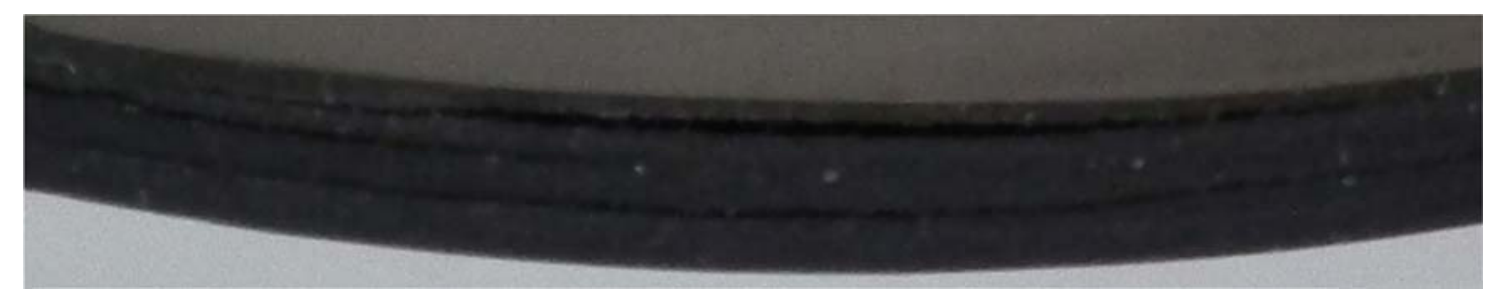

Figure 61: Delamination in the cylinder free edge - type B cylinder (31 J).

Table 24: Ratio between elastic energy $\left(E_{e}\right)$ and dissipated energy $\left(E_{d}\right)$ - Type B cylinders $(31 \mathrm{~J})$

\begin{tabular}{cc}
\hline Coupon & $\mathbf{E}_{\mathbf{e}} / \mathbf{E}_{\mathbf{d}}$ \\
\hline 1 & 1.30 \\
\hline 2 & 1.33 \\
\hline 3 & 1.15 \\
\hline 4 & 1.44 \\
\hline Average & 1.30 \\
\hline
\end{tabular}

\subsubsection{Results for Cylinder Type C}

Figure 62 shows the force vs. time and displacement vs. time for type C cylinders under $8.4 \mathrm{~J}$ impact test. In this case, as for type B $8.4 \mathrm{~J}$ impact test, the maximum force occurs almost at the same point (response delay of $0.4 \mathrm{~ms}$ ). Also, the test results for all coupons show good repeatability. 
Type C - Force vs. Time and Displacement vs. Time

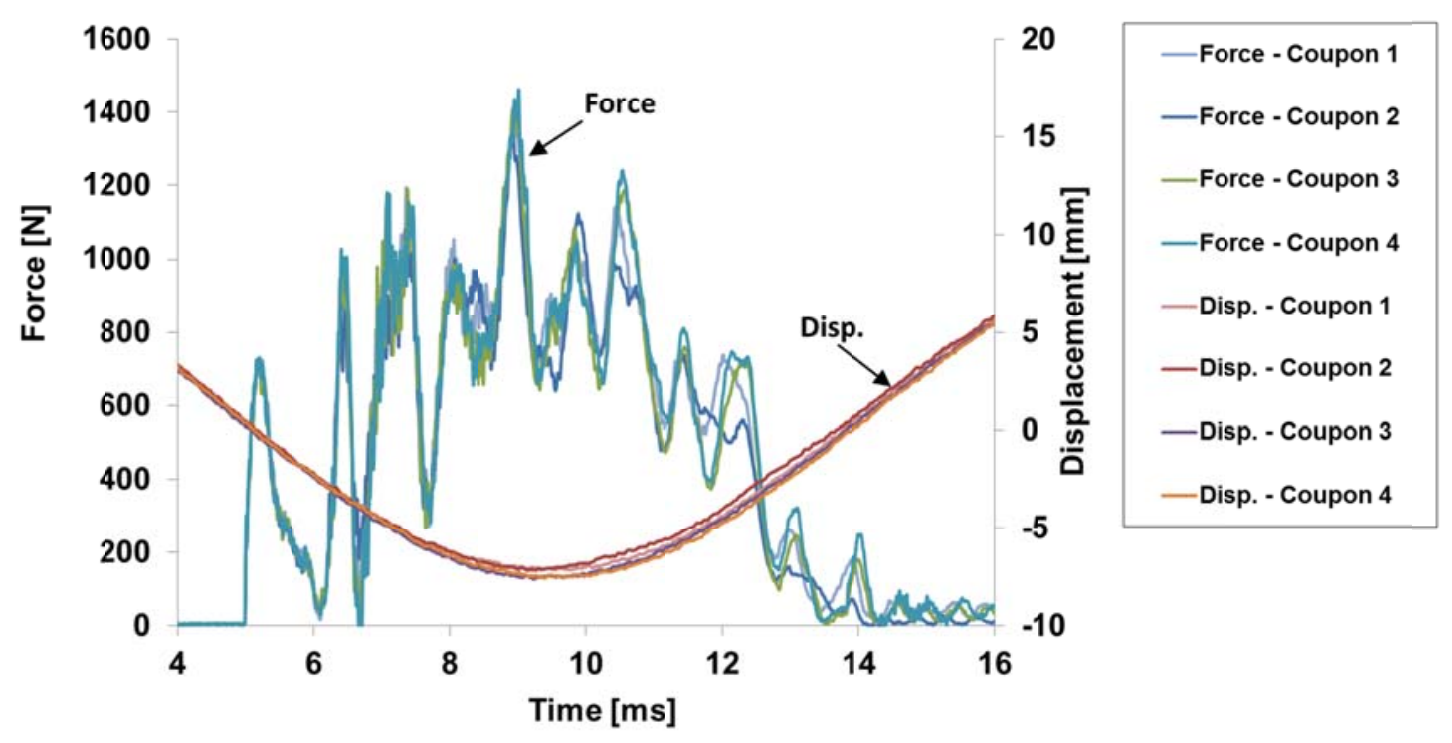

Figure 62: Type C (8.4 J) - force vs. time and displacement vs. time.

Figure 63 shows the strain vs. time and force vs. time results for type C cylinders under $8.4 \mathrm{~J}$ impact. Unfortunately, only the strain-gage data for coupons 1 and 3 have been recorded. Maximum force peak occurs close to the minimum strain with a small response delay of 0.4 ms. 


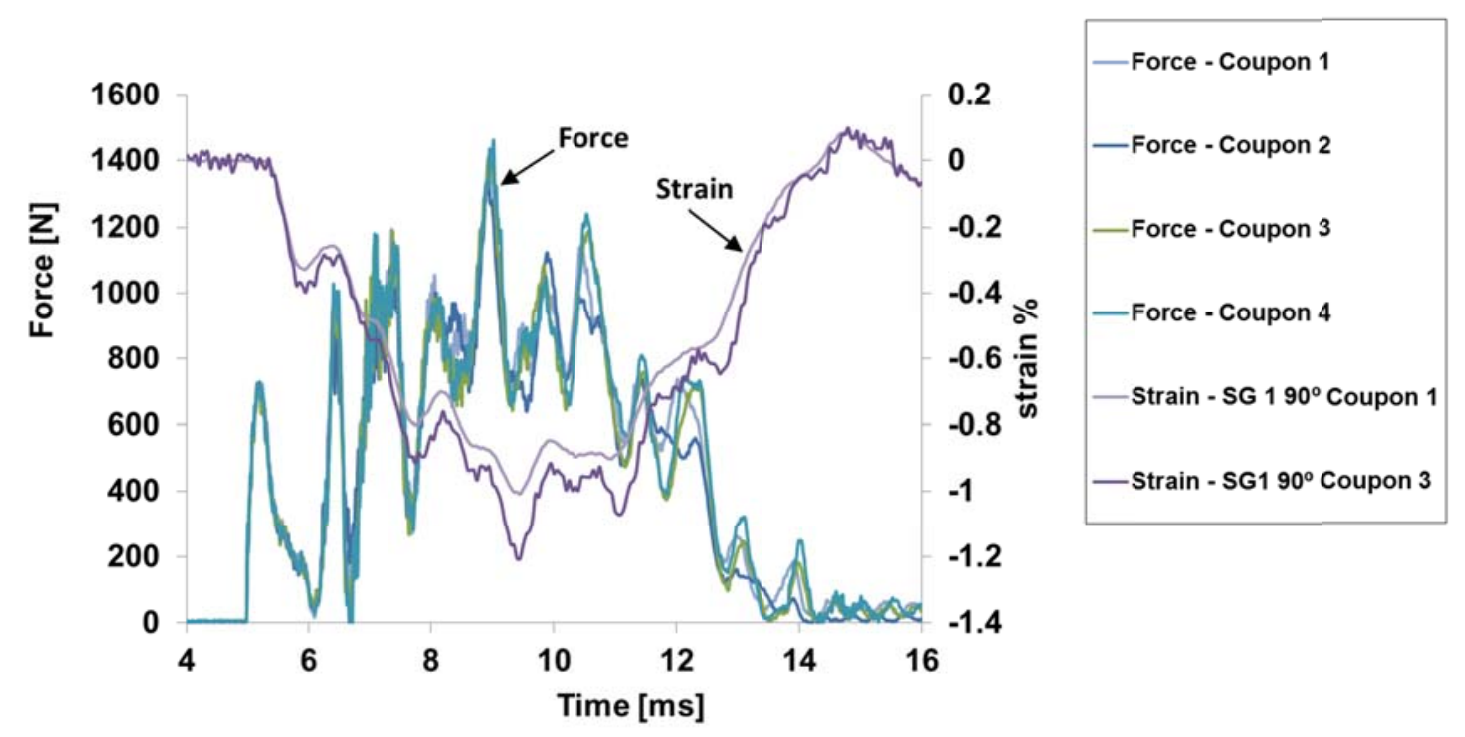

Figure 63: Type C (8.4 J) - force vs. time and strain vs. time.

Table 25 shows the maximum displacement and minimum strain values for type $\mathrm{C}$ cylinders under $8.4 \mathrm{~J}$ impact.

Table 25: Maximum force, displacement and strain for type C cylinders (8.4 J).

\begin{tabular}{cccc}
\hline Coupon & $\begin{array}{c}\text { Maximum force } \\
\text { peak [N] }\end{array}$ & $\begin{array}{c}\text { Maximum displacement } \\
{[\mathbf{m m}] \text { (absolute value) }}\end{array}$ & Minimum strain [\%] \\
\hline 1 & 1401.4 & 7.1 & -1.009 \\
\hline 2 & 1335.4 & 7.2 & - \\
\hline 3 & 1411.1 & 7.6 & - \\
\hline 4 & 1462.4 & 7.6 & -1.107 \\
\hline Average & 1402.6 & 7.4 & 0.139 \\
\hline Standard Deviation & 52.1 & 0.25 & \\
\hline
\end{tabular}

Figure 64 shows the measured strains for type $\mathrm{C}$ cylinders $(8.4 \mathrm{~J})$. The delay of the response between strain gages " 1 " and " 2 " is around $1.6 \mathrm{~ms}$ (Figure 64). This is the biggest value detected over all cylinder types. Table 26 shows the response delay between strain gages " 1 " and " 2 " for all type C cylinders under $8.4 \mathrm{~J}$ impact. 


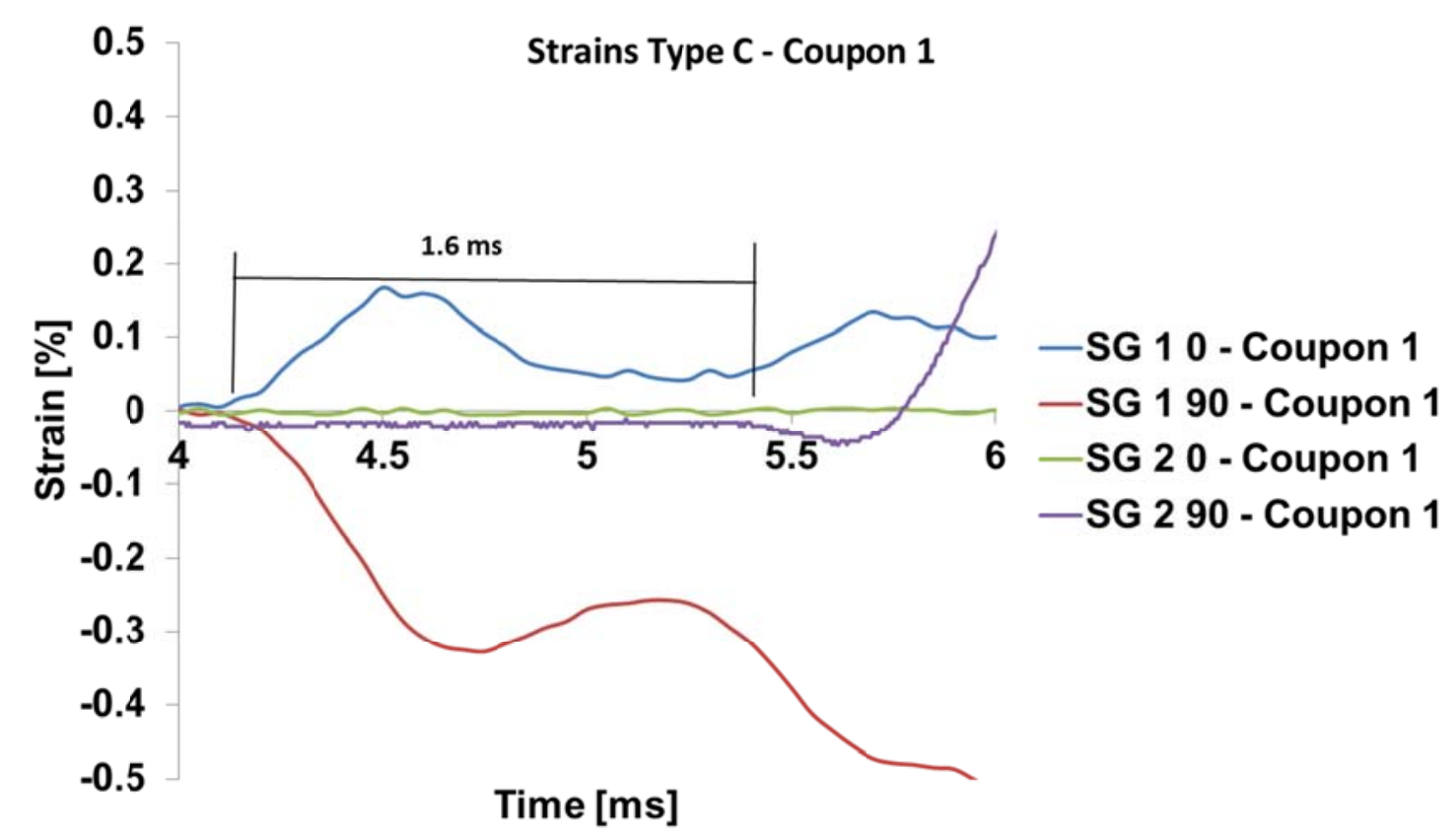

Figure 64: Type $\mathrm{C}$ coupon 3 strains

Table 26: Delay between strain gage 1 and 2 - Type C cylinders ( $8.4 \mathrm{~J})$.

\begin{tabular}{cc}
\hline Coupon & Delay [s] \\
\hline 1 & 0.00157 \\
\hline 2 & 0.00175 \\
\hline 3 & 0.00154 \\
\hline 4 & 0.00173 \\
\hline Average & 0.00165 \\
\hline
\end{tabular}

As observed for type A and B cylinders under $8.4 \mathrm{~J}$ impact, the impactor transfers its kinetic energy in a constant slope line as shown in Figure 65. As identified for type B, type C cylinders restored almost all energy, once the elastic energy is around 10 times the dissipated energy (Table 15). Thus, it is possible to consider that all energy has been restored to the impactor and the impact event did not cause any considerable damage, confirming the results obtained by $\mathrm{C}$-scan analysis. 


\section{Type C - Kinetic Energy}

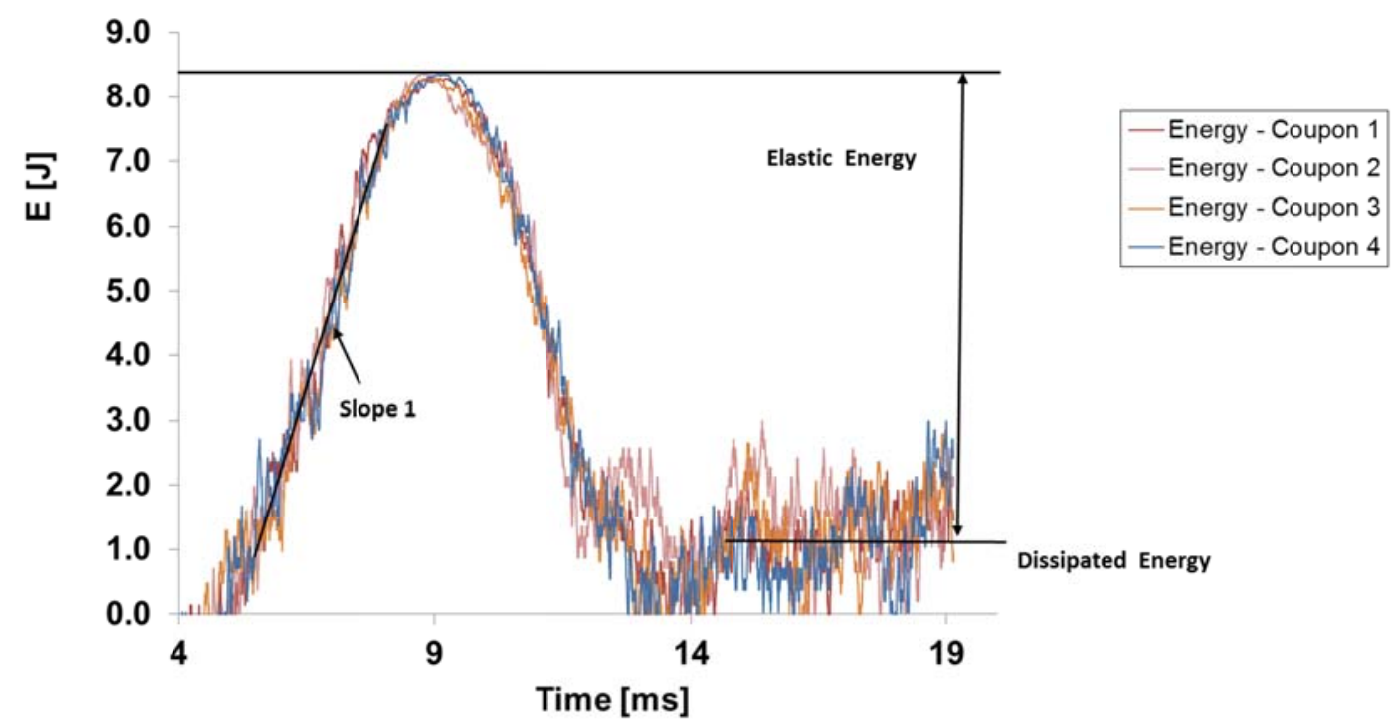

Figure 65: Kinetic energy balance between impactor and type $\mathrm{C}$ coupons for $8.4 \mathrm{~J}$ impact test.

Table 27: Ratio between elastic energy $\left(E_{e}\right)$ and dissipated energy $\left(E_{d}\right)-$ Type $C$ cylinders $(8.4 \mathrm{~J})$

\begin{tabular}{cc}
\hline Coupon & $\mathbf{E}_{\mathbf{e}} / \mathbf{E}_{\mathbf{d}}$ \\
\hline 1 & 10.84 \\
\hline 2 & 6.59 \\
\hline 3 & 8.21 \\
\hline 4 & 15.69 \\
\hline Average & 10.33 \\
\hline
\end{tabular}

Figure 66 shows force vs. time and displacement vs. time results for type C cylinders under $31 \mathrm{~J}$ impact test. The same evaluation for type A and B cylinders is applicable to type C cylinders. Besides, this configuration reaches the highest value of force peak over all cylinders types. Delaminations, matrix damage and indentation marks (inelastic deformation) are also detected for type $\mathrm{C}$ cylinders. 


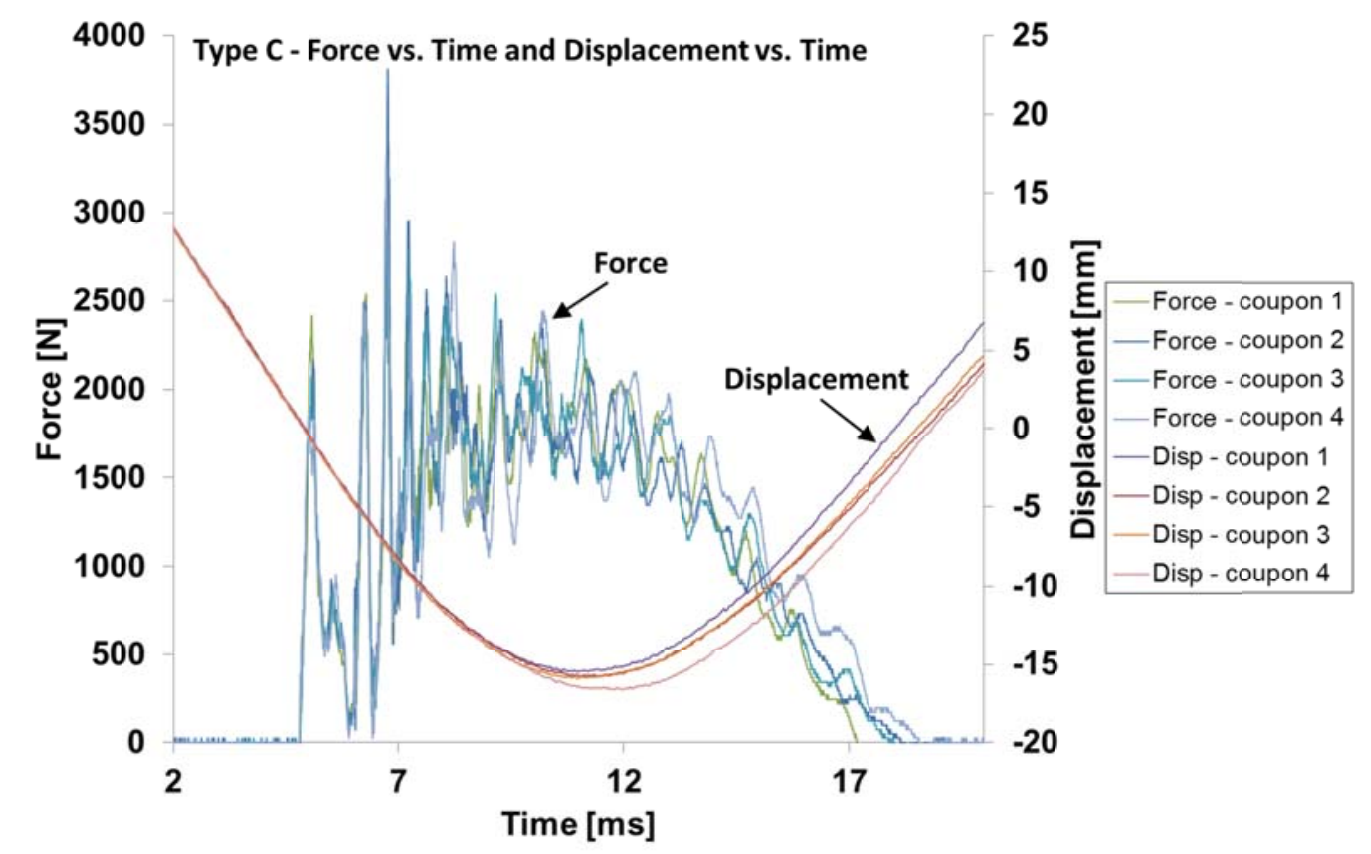

Figure 66: Type C (31 J) - force vs. time and displacement vs. time

Table 28 summarizes the results for coupons type $\mathrm{C}$ under $31 \mathrm{~J}$ impact. In this case, the displacement standard deviation is $3.1 \%$. Hence, this is the highest value, considering all cylinder responses. Also, the force standard deviation is $11.0 \%$ of the average value. Again, there is no correlation between maximum force and displacement.

Table 28: Maximum force and displacement for type $\mathrm{C}$ cylinders $(31 \mathrm{~J})$

\begin{tabular}{ccc}
\hline Coupon & $\begin{array}{c}\text { Maximum force } \\
\text { peak [N] }\end{array}$ & $\begin{array}{c}\text { Maximum displacement [mm] } \\
\text { (absolute value) }\end{array}$ \\
\hline 1 & 3393.6 & 15.5 \\
\hline 2 & 3808.6 & 15.8 \\
\hline 3 & 2905.3 & 15.9 \\
\hline 4 & 3320.3 & 16.7 \\
\hline Average & 3356.9 & 16.0 \\
\hline Standard Deviation & 370.0 & 0.5 \\
\hline
\end{tabular}

Considering the energy balance between the impactor and the type $\mathrm{C}$ cylinders under $31 \mathrm{~J}$ impact tests, the impactor transfers its kinetic energy to the coupons in a bilinear way (Figure 67). This behavior has been identified for type B cylinders, but not for type A ones. The maximum force peak occurs at $6.8 \mathrm{~ms}$, the maximum displacement occurs at $11.7 \mathrm{~ms}$ and a 
response delay of $5.0 \mathrm{~ms}$ has been observed. Regarding the restored energy, this cylinder configuration dissipates $77 \%$ of the elastic energy (Table 29) and this result is almost the same as that evaluated for type B configuration.

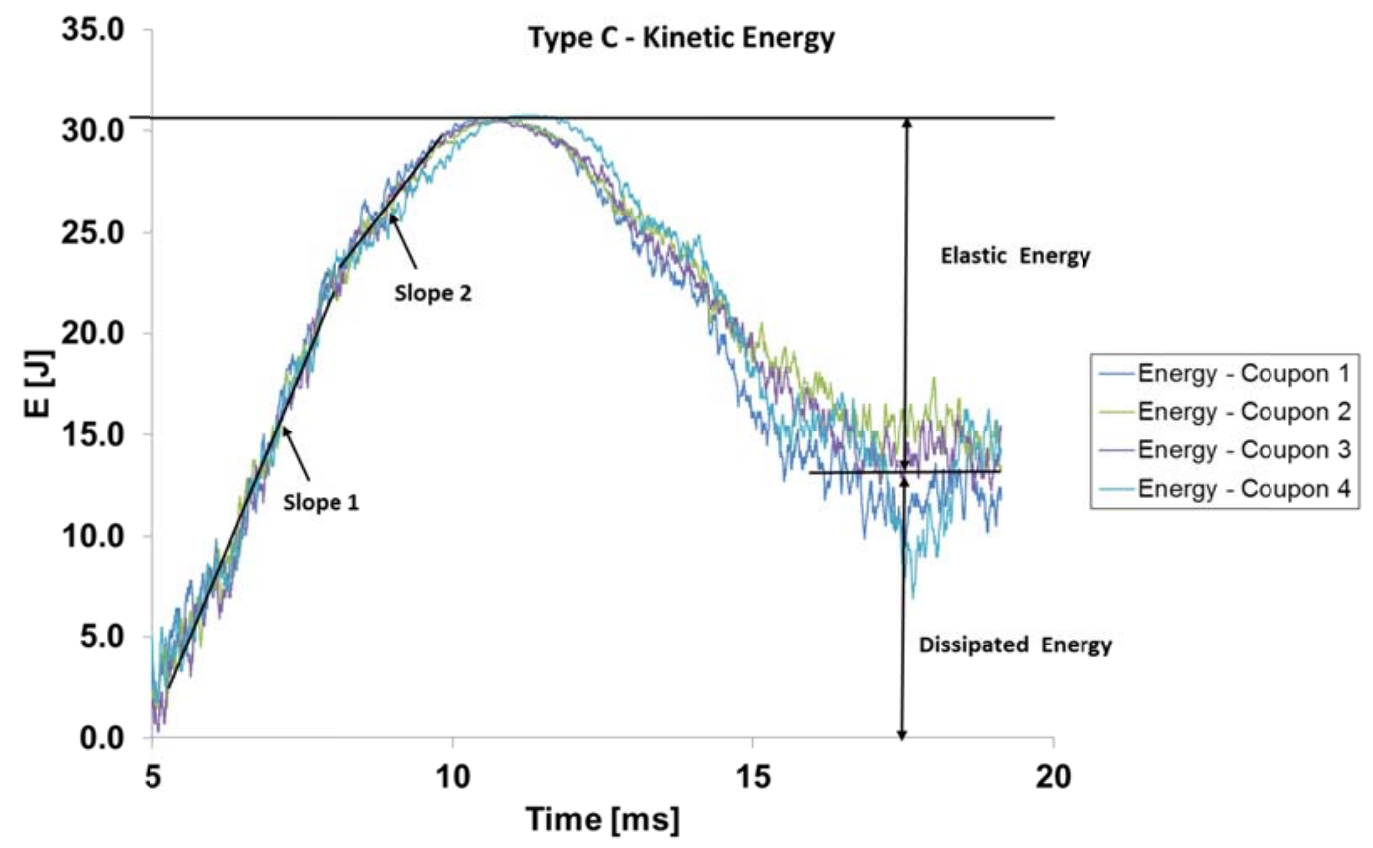

Figure 67: Kinetic energy balance between impactor and type $\mathrm{C}$ coupons for $31 \mathrm{~J}$ impact test.

As observed in other $31 \mathrm{~J}$ impact tests, the behavior of the kinetic energy balance (Figure 67) has shown a pattern, which confirms the influence of the damage process in transferring the energy from the coupon to the impactor.

Table 29: Ratio between elastic energy $\left(E_{e}\right)$ and dissipated energy $\left(E_{d}\right)$ - Type C (31 J)

\begin{tabular}{cc}
\hline Coupon & $\mathbf{E}_{\mathrm{e}} / \mathbf{E}_{\mathbf{a}}$ \\
\hline 1 & 1.76 \\
\hline 2 & 1.05 \\
\hline 3 & 1.18 \\
\hline 4 & 1.46 \\
\hline Average & 1.30 \\
\hline
\end{tabular}




\subsubsection{Comparison of Results}

Type C cylinders present higher load peaks (Table 30 and Table 31) than other cylinder types. However, in $8.4 \mathrm{~J}$ impact tests, the average values of forces were close to each other over all cylinder types.

Table 30: Maximum force peak (8.4 J).

\begin{tabular}{ccc}
\hline Cylinder Type & Maximum peak & Difference \\
& load [N] & $\frac{V_{\text {Typei }}-V_{\text {Type A }}}{V_{\text {Ttype A }}} \cdot 100$ \\
\hline Type A & 1368.4 & - \\
\hline Type B & 1301.7 & $-4.9 \%$ \\
\hline Type C & 1402.6 & $2.5 \%$ \\
\hline
\end{tabular}

Table 31 shows the values of the maximum load peak and the differences among type A and the other types. This table shows that for the same impact energy, type $C$ cylinders have an average force $40.7 \%$ higher than type A cylinders, and type B cylinders have an average force $13.6 \%$ higher than type A cylinders. These differences did not occur in $8.4 \mathrm{~J}$ impact tests.

Table 31: Maximum force peak (31 J).

\begin{tabular}{ccc}
\hline Cylinder Type & Maximum peak & Difference \\
& load [N] & $\frac{V_{\text {Typei }}-V_{\text {Type A }}}{V_{\text {Ttype A }}} \cdot 100$ \\
\hline Type A & 2385.6 & - \\
\hline Type B & 2710.0 & $13.6 \%$ \\
\hline Type C & 3356.9 & $40.7 \%$ \\
\hline
\end{tabular}

Type B cylinders present higher displacement (Table 32 and Table 34) than other cylinder types. All comments for Type A cylinders can also be considered for Type C cylinders. Average results were rather close both for $8.4 \mathrm{~J}$ and $31 \mathrm{~J}$ impact tests. On the other hand, the strain data present a considerable dispersion as shown in Table 32. 
Table 32: Maximum displacement (absolute values) for $8.4 \mathrm{~J}$.

\begin{tabular}{|c|c|c|}
\hline Cylinder Type & $\begin{array}{l}\text { Maximum absolut } \\
\text { displacement [mm] }\end{array}$ & $\begin{array}{c}\text { Difference } \\
\frac{V_{\text {Typei }}-V_{\text {Type A }}}{V_{\text {Ttype A }}} \cdot 100\end{array}$ \\
\hline Type A & 7.2 & - \\
\hline Type B & 7.7 & $6.9 \%$ \\
\hline Type C & 7.4 & $2.8 \%$ \\
\hline
\end{tabular}

Table 33: Minimum strain (strain gage $1-90^{\circ}$ ) for $8.4 \mathrm{~J}$.

\begin{tabular}{ccc}
\hline Cylinder Type & Minimum strain & Difference \\
& {$[\%]$} & $\frac{V_{\text {Typei }}-V_{\text {Type A }}}{V_{\text {Ttype A }}} \cdot 100$ \\
\hline Type A & -0.761 & - \\
\hline Type B & -1.022 & $34.3 \%$ \\
\hline Type C & -1.107 & $45.5 \%$ \\
\hline
\end{tabular}

Table 34: Maximum displacement (absolute values) for $31 \mathrm{~J}$.

\begin{tabular}{ccc}
\hline Cylinder Type & $\begin{array}{c}\text { Maximum absolut } \\
\text { displacement } \\
{[\mathbf{m m}]}\end{array}$ & $\begin{array}{c}\text { Difference } \\
\frac{V_{\text {Typei }}-V_{\text {Type A }}}{V_{\text {Type A }}} \cdot 100\end{array}$ \\
\hline Type A & 16.2 & - \\
\hline Type B & 16.4 & $1.2 \%$ \\
\hline Type C & 16.0 & $-1.2 \%$ \\
\hline
\end{tabular}

Regarding the damaged area, type A cylinders were more damaged than the others, mainly in the internal surface. The other cylinders types did not show damage in the internal surface. Delaminations, matrix damage and indentation marks have also been detected in type $\mathrm{C}$ cylinders.

The response delay in type A cylinders has the lowest value and type $\mathrm{C}$ produces the highest value. Type A has the lay-up with fiber angles close to $90^{\circ}$, which are stiffer for hoop stress than type B and C. Besides, type C has only two layers with $90^{\circ}$, what means that it is 
more flexible in the hoop direction. Therefore, the stress wave propagates faster in type A than in types $\mathrm{B}$ and $\mathrm{C}$, considering the hoop direction.

Table 35 shows the average delay value between the strain gages 1 and 2 for cylinders type $\mathrm{A}, \mathrm{B}$ and $\mathrm{C}$.

Table 35: Delay between strain gages for 8.4 J.

\begin{tabular}{ccc}
\hline Cylinder Type & Delay [s] & $\frac{V_{\text {Typei }}-V_{\text {Type A A }}}{V_{\text {Ttype A }}} \cdot 100$ \\
\hline Type A & & - \\
\hline Type B & 0.00127 & $22.7 \%$ \\
\hline Type C & 0.00155 & $29.9 \%$ \\
\hline
\end{tabular}

Considering the average values of ratio between elastic to dissipated energy under $8.4 \mathrm{~J}$ impact for all cylinders types (Table 36), the differences among type B, C and type A are very significant. Type B and C cylinders restore almost all energy to the impactor and almost no damage occurs. On the other hand, type A cylinders restore much less energy due to internal damage process, which has not been detected by the C-scan analysis.

Table 36: Energy ratio for $8.4 \mathrm{~J}$.

\begin{tabular}{ccc}
\hline Cylinder Type & $\mathbf{E}_{\mathrm{e}} / \mathbf{E}_{\mathbf{d}}$ & $\left|\frac{V_{\text {Typei }}-V_{\text {Type A }}}{V_{\text {Ttype A }}}\right| \cdot 100$ \\
& & - \\
\hline Type A & 1.51 & $362.2 \%$ \\
\hline Type B & 6.98 & $584.1 \%$ \\
\hline Type C & 10.33 & \\
\hline
\end{tabular}

Finally, there is a comparison between the average values of elastic to dissipated energy ratio under $31 \mathrm{~J}$ impact tests for all cylinders types (Table 37 ). The difference between type $\mathrm{B}$, $\mathrm{C}$ and $\mathrm{A}$ is not as pronounced as shown by $8.4 \mathrm{~J}$ impact test. At $31 \mathrm{~J}$ energy level, type $\mathrm{B}$ and $\mathrm{C}$ cylinders restore more energy to the impactor than they dissipate, and type A cylinders 
restore less energy to the impactor. All cylinder types show several failure mechanisms after the impact under $31 \mathrm{~J}$.

Table 37: Energy ratio for $31 \mathrm{~J}$.

\begin{tabular}{ccc}
\hline Cylinder Type & $\mathbf{E}_{\mathbf{e}} / \mathbf{E}_{\mathbf{d}}$ & $\left|\frac{V_{\text {Typei }}-V_{\text {Type A }}}{V_{\text {Ttype A }}}\right| \cdot 100$ \\
\hline Type A & & - \\
\hline Type B & 0.98 & $32.6 \%$ \\
\hline Type C & 1.30 & $32.6 \%$ \\
\hline
\end{tabular}

\subsubsection{IMPACT TESTS CONCLUSIONS}

The results of impact tests in a set of cylinders show that the stacking sequence is a very important parameter in the cylinder impact behavior. Type A cylinders are more susceptible to damage than types B or C.

Although $\mathrm{C}$-scan has not detected any damage for type A cylinders under $8.4 \mathrm{~J}$ impact test, some other phenomena could be evaluated by using the graphics. For instance, the delay between the maximum force peak and the maximum displacement and the delay between the maximum force peak and the minimum strain is very similar to the delay pattern shown by type A cylinders under $31 \mathrm{~J}$ impact test. Thus this off-set between maximum force value and maximum displacement could work as an indicator of damage. Furthermore, the strongest indication of damage on type A cylinder caused by $8.4 \mathrm{~J}$ impact test is the ratio between elastic and dissipated energy, once the energy ratio shown by type A cylinders was different from other cylinders types.

Cylinders type $\mathrm{B}$ and $\mathrm{C}$ have not dissipated a considerable amount of energy due to the impact test. Besides the delay between their maximum force value and maximum displacement (or minimum strain) has been much lower than that for type A cylinders.

Considering $31 \mathrm{~J}$ impact test, all coupons have shown several damaged mechanisms, such as delaminations, matrix cracking and dent marks. Furthermore, for all types, the off-set between the maximum force and maximum displacement has been considerable. Therefore, 
the mentioned delay can also be regarding a damage indicator, since it has not been detected in the elastic response obtained from $8.4 \mathrm{~J}$ impact tests (cylinders type $\mathrm{B}$ and $\mathrm{C}$ ).

Furthermore, for $31 \mathrm{~J}$ impact energy, the kinetic energy has been transferred from the impactor to the cylinders in at least two slope curves. This change of slope indicates that a more severe mechanism of damage takes place. It is important to highlight that this behavior has not been detected in all cylinders types under $8.4 \mathrm{~J}$ impact test. In those cases, the energy from the impactor has been transferred to the cylinders in one slope curve.

Finally, it has also been shown that the lay-up does not have an important effect neither in the maximum displacement nor in the total impact time in all investigated cylinder types at the same energy level. 


\section{Finite ELEMENT ANALYSES}

After the development of the damage model and the identification of the model parameters, it was possible to implement the entire mathematical formulation with all parameters adequately specified.

In order to evaluate the material model, it is necessary to perform nonlinear material FE (Finite Element) analyses by using $\mathrm{ABAQUS}^{\mathrm{TM}}$ and the UMAT or VUMAT subroutines. Moreover, the step size and mesh density are very important in the convergence process of the numerical analyses. Thus, step size and mesh convergence tests have been performed to find the optimum model performance to simulate the damage process. Also, as observed in the experiments, the failure mechanisms occurred in small displacements applied to the coupons; so nonlinear geometric effects have not been considered neither in the FE tensile nor in four point bending analyses. On the other hand, geometric non-linearity has been used for both compression and impact analysis.

\subsection{Tensile Results}

The FE model for $\left[0^{\circ}\right]_{10}$ tensile tests had the same dimensions of the coupons used for the experiments, but only the length between the grips was modelled (Figure 68), once the model geometry is simple and the results are not affected by those boundary conditions. For the FE mesh, 4-node fully integrated homogeneous shell elements have been used (defined in $\mathrm{ABAQUS}^{\mathrm{TM}}$ as $\mathrm{S} 4$, which each layer was $0.2 \mathrm{~mm}$ thick and had three integration points along the thicknes)s. Also, the material properties for each layer have been obtained by the experiments. The boundary conditions for tensile tests $\left(0^{\circ}\right.$ and $90^{\circ}$ coupons $)$ were applied in order to simulate the restrictions imposed by the grips and the displacement controlled in one extremity of the coupon. The terms $U x, U y$ and $U z$ are the displacements in $x$ (red arrow), $y$ (green arrow) and z (blue arrow), respectively. The terms Rx, Ry and Rz are the rotations around $\mathrm{x}, \mathrm{y}$ and $\mathrm{z}$, respectively (Figure 68).

Since the bending moment to straighten the almost flat coupon by the machine fixtures does not produce a high stress state on the coupon, this effect might be neglected in the the FE analyses. 


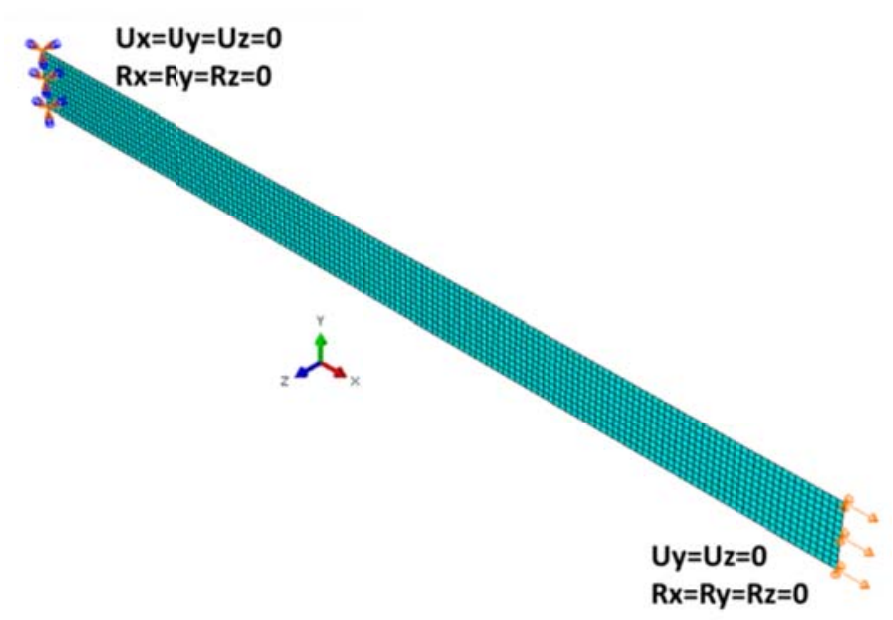

Figure 68: FE model for tensile tests $\left(0^{\circ}\right.$ coupons).

To avoid numerical problems during the FE static analyses, the step size has been set to be automatic in the program. Then in order to study the step size sensibility, only the maximum step size limit has been modified. After that, different structured meshes of quadrilateral elements with six different element sizes (side length), using the maximum step size have been evaluated (Figure 69). The FE model, which shows better performance, had the combination of $0.5 \mathrm{~mm}$ element size (the largest s side of the regular four-side element) and 0.01 for maximum step size (i.e. $1 \%$ of the maximum prescribed displacement for each increment), regarding simulation time and accuracy.

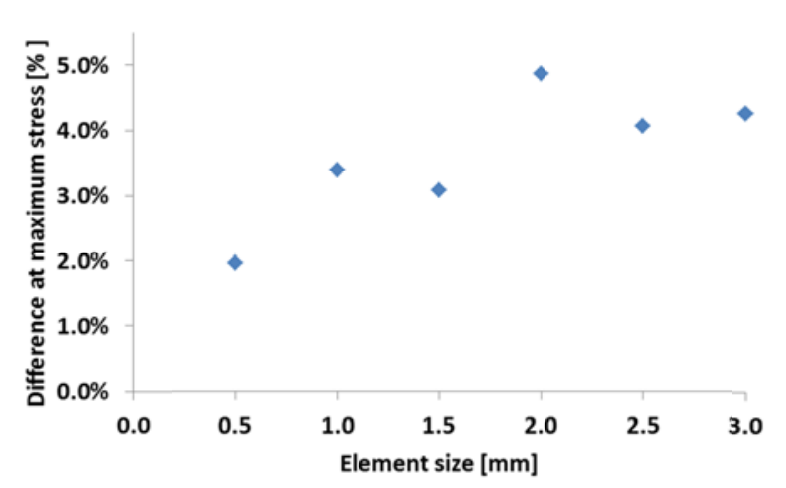

(a)

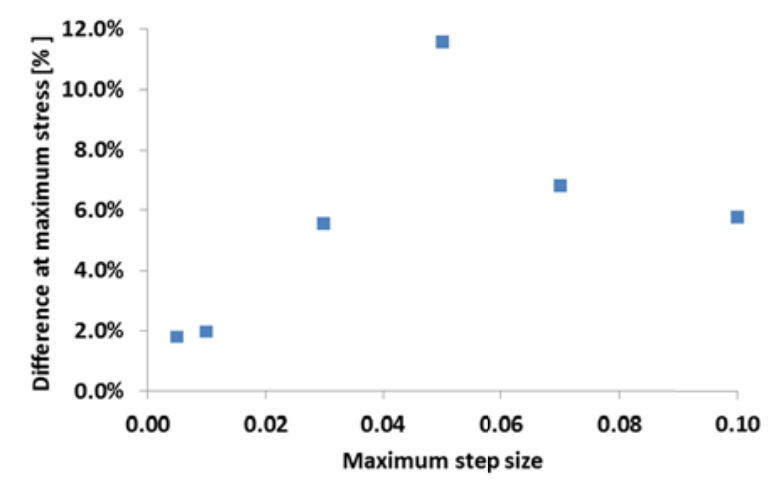

(b)

Figure 69: Tensile of $0^{\circ}$ coupons - finite element mesh sensitivity analysis (a) and step size analysis (b) Difference $=\mid($ Experiments average max imum stress - Simulations $) /$ Experiments average $\max$ imum stress $\mid \%$ 
The difference is calculated regarding the average of the tensile maximum stress reached during the experiments for each tested coupon and the maximum stress reached by the FE model during the simulations for each case (element size and step size).

Both the experimental and numerical results for $0^{\circ}$ coupons under tensile loads have been presented in Figure 70. As expected, a linear elastic fragile behavior occurs in the experiments. It is observed that the FE model had good correlation to the experimental curves. Also, no damage had been detected before fiber breakage as verified during the quasi-static cyclic tests. This is confirmed by the cyclic curves, which do not show any stiffness degradation for most of the coupons (Figure 70). In fact, just for one coupon (CDP 0-1), it was possible to verify that some fibers fail at high load level. This is confirmed by the stress vs. strain graph (red line) as shown by a sudden drop in the stress level (Figure 66).

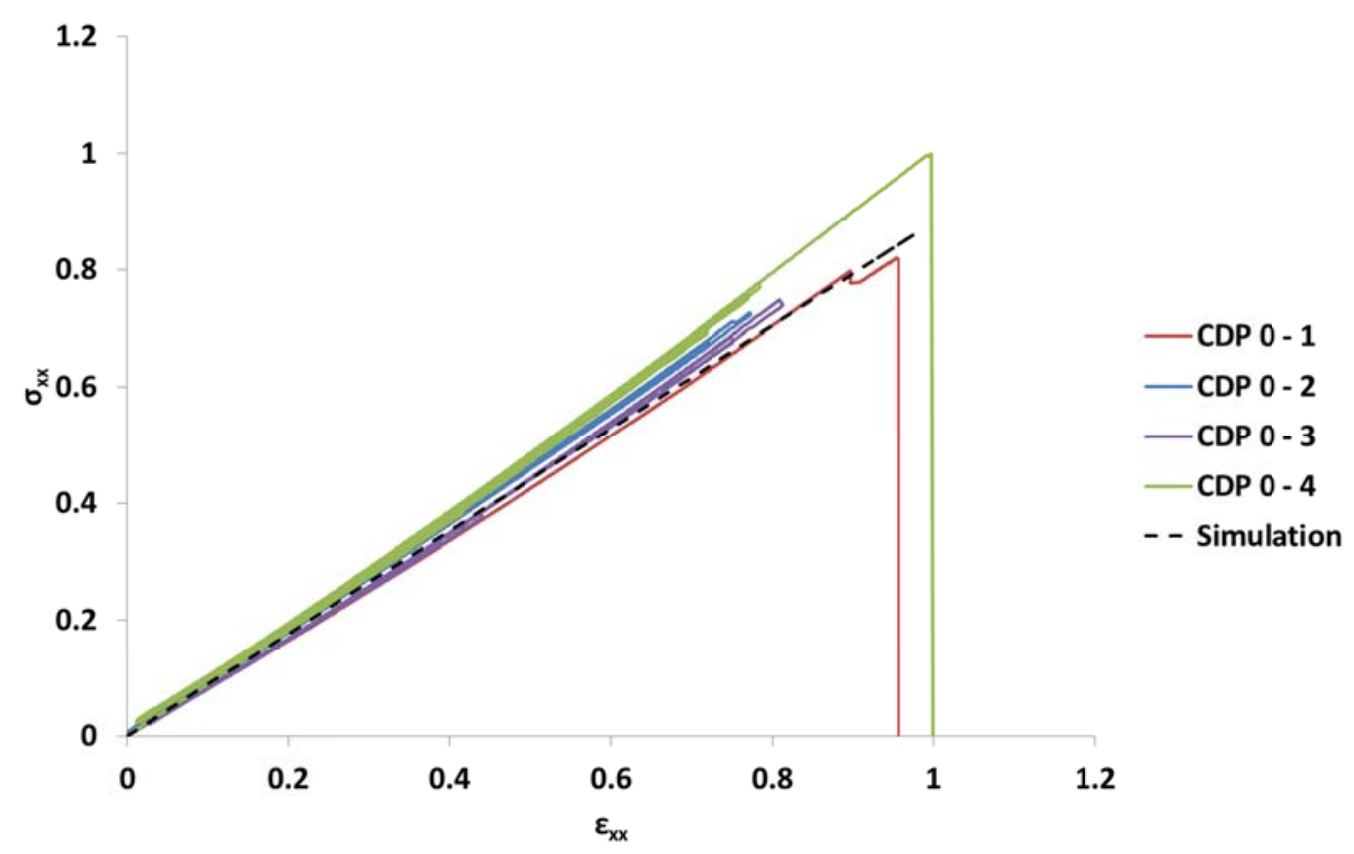

Figure 70: Normalized stress vs. strain for tensile $\left[0^{\circ}\right]_{10}$ results - numerical and experimental analyses.

Table 38 summarises the experimental stresses and strains as well as the numerical results for tensile $\left[0^{\circ}\right]_{10}$ coupons. Regarding the average values, the relative difference between numerical simulation and experimental analyses is $4.88 \%$ for stress and $10.11 \%$ for strain. 
Table 38: Tensile $\left[0^{\circ}\right]_{10}$ normalized results for numerical and experimental analyses.

\begin{tabular}{ccc}
\hline Coupon & Normalized Maximum Stress & Normalized Maximum Strain \\
\hline CDP 0-1 & 0.82 & 0.96 \\
\hline CDP 0-2 & 0.73 & 0.78 \\
\hline CDP 0-3 & 0.75 & 0.82 \\
\hline CDP 0-4 & 1.00 & 1.00 \\
\hline Experiments average & 0.82 & 0.89 \\
\hline Numerical Prediction & 0.86 & 0.98 \\
\hline Difference \% & 4.88 & 10.11 \\
\hline
\end{tabular}

Difference $=\mid($ Experiments average - Numerical Prediction $) /$ Experiments average $\mid \%$

As carried out for $0^{\circ}$ tensile finite element model, the finite element model for $90^{\circ}$ coupons under tensile has been developed by using structured meshes with quadrilateral elements Again, six different element sizes (side length) using maximum step size were evaluated (Figure 71). In this case, the FE model demonstrated a step size dependency greater than that for $0^{\circ}$ coupons. The FE model, which shows better performance, had the combination of $0.5 \mathrm{~mm}$ element size and 0.005 for maximum step size (i.e. $0.5 \%$ of the maximum prescribed displacement for each increment), regarding simulation time and accuracy.

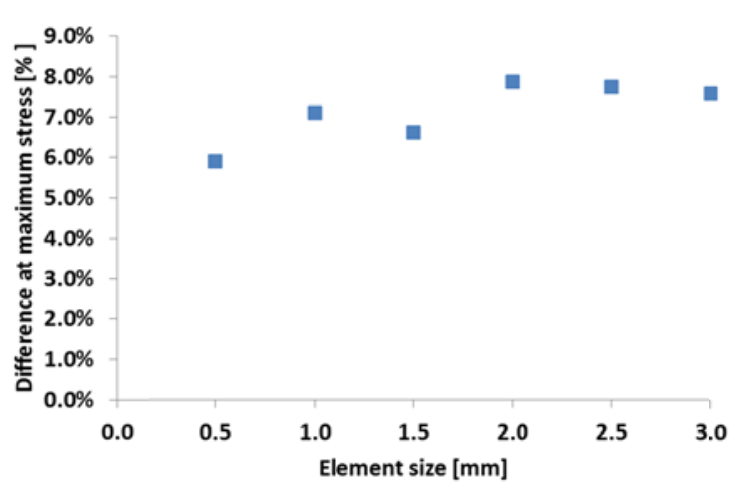

(a)

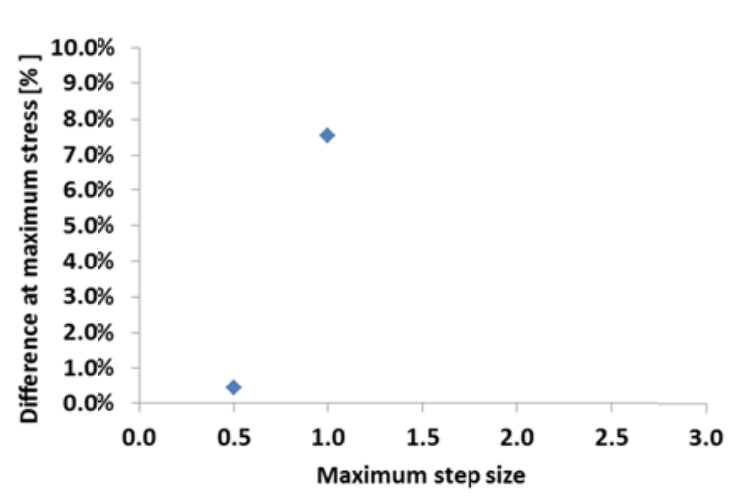

(b)

Figure 71: Tensile of $90^{\circ}$ coupons - finite element mesh sensitivity analysis (a) and step size analysis(b)

Difference $=\mid($ Experiments average max imum stress - Simulations $) /$ Experiments average $\max$ imum stress $\mid \%$ 
The difference is calculated regarding the average of the tensile maximum stress reached during the experiments for each tested coupon and the maximum stress reached by the FE model during the simulations for each case (element size and step size).

For $90^{\circ}$ coupons under tensile load, the stress results are shown in Figure 72. A significant scatter is observed among the strength values in the experimental results. However, there is a further fair correlation for the stiffness in the experimental curves. The numerical model shows an acceptable performance to simulate the $90^{\circ}$ coupons under tensile tests, because the matrix damage process in the $90^{\circ}$ coupons is complex. This process starts with initiation of micro cracks due to tensile loads. Since the load magnitude increases progressively, there is propagation of these cracks. In order to simulate this process, the damage parameter $\mathrm{d}_{2}$ should evolve and the evolution $\mathrm{d}_{2}$ is accounted for the effective stress (EQ. (22) - see Chapter 2); as a consequence the strain energy is modified due to the damage evolution. Moreover, as commented in the mathematical formulation of the model, the brittle failure of the matrix occurs when the strain energy reaches a critical value identified via experimental analysis.

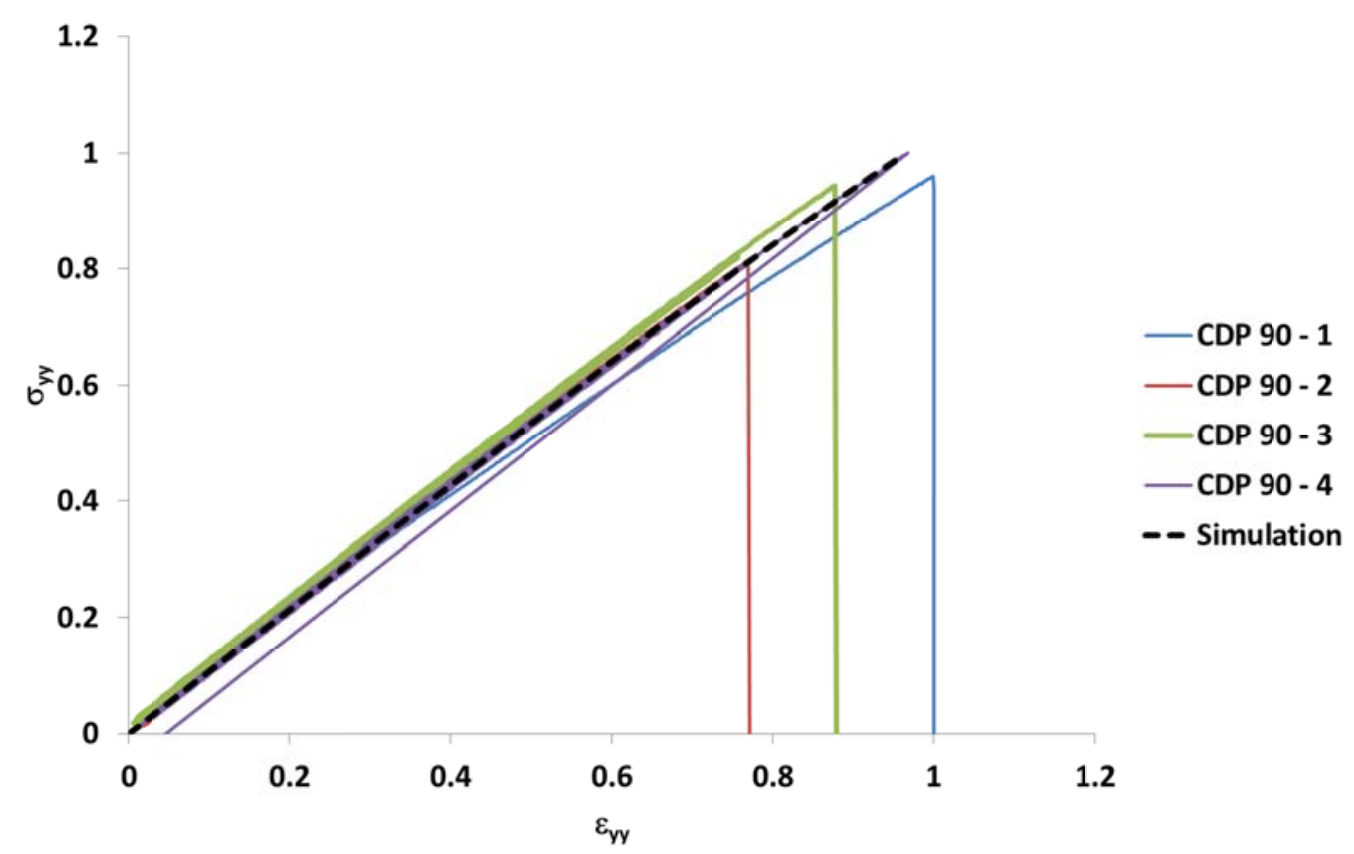

Figure 72: Normalized stress vs. strain for tensile $\left[90^{\circ}\right]_{10}$ results - numerical and experimental analyses.

Table 39 summarises the experimental stresses and strains as well as the numerical results for $90^{\circ}$ coupons under tensile loads. The relative difference between the average of the experimental strength and numerical prediction is $4.30 \%$ for stress and $4.44 \%$ for strain. 
Table 39: Tensile $\left[90^{\circ}\right]_{10}$ normalized results for numerical and experimental analyses.

\begin{tabular}{ccc}
\hline Coupon & Normalized Maximum Stress & Normalized Maximum Strain \\
\hline CDP 90-1 & 0.96 & 1.00 \\
\hline CDP 90-2 & 0.81 & 0.77 \\
\hline CDP 90-3 & 0.94 & 0.88 \\
\hline CDP 90-4 & 1.00 & 0.97 \\
\hline Experiments Average & 0.93 & 0.90 \\
\hline Numerical Prediction & 0.97 & 0.94 \\
\hline Difference \% & 4.30 & 4.44 \\
\hline
\end{tabular}

Difference $=\mid($ Experiments average - Numerical Prediction $) /$ Experiments average $\mid \%$

The next analyses have been performed in order to evaluate the model for a more complex stress state, by using the off-axis coupons. The finite element model had the same characteristics as those used for tensile tests (boundary conditions and element type), but it had the same dimensions as those used for off-axis compression coupons.

The investigated mesh and step size are shown in Figure 73. For $5^{\circ}$ coupons under off-axis tensile. The mesh density as well as the step size had a significant influence on the simulations results. The best combination for this case was $0.5 \mathrm{~mm}$ for element size and 0.005 for maximum step size (i.e. $0.5 \%$ of the prescribed displacement for each increment).

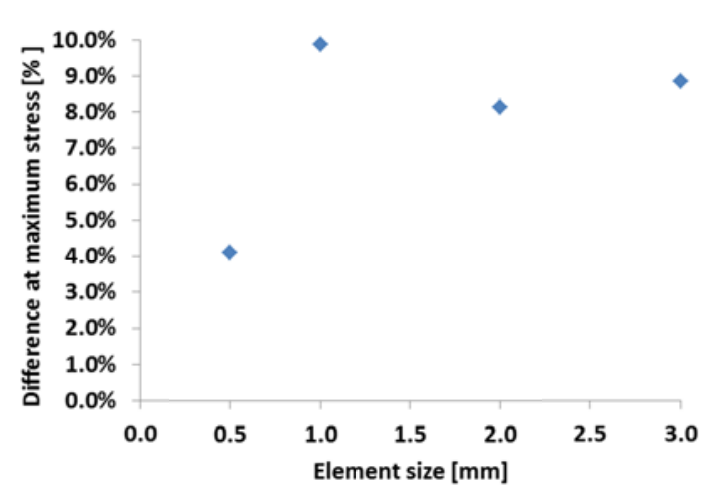

(a)

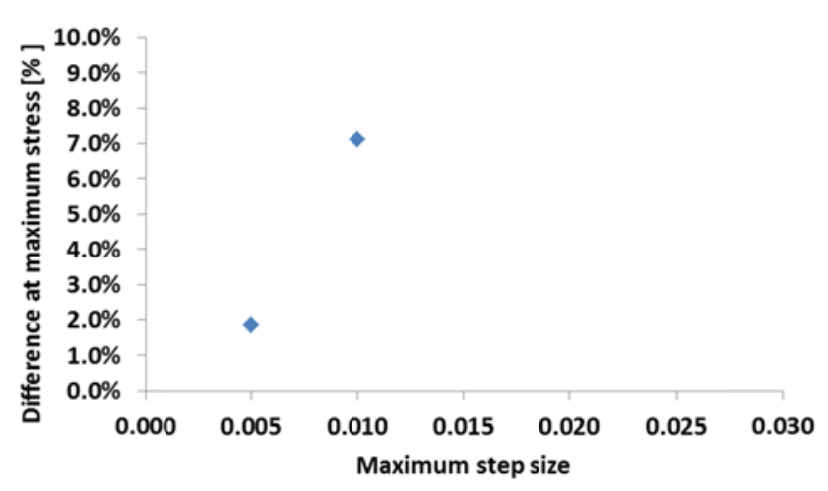

(b)

Figure 73: Tensile $5^{\circ}$ coupons - finite element mesh sensitivity analysis (a) and step size analysis(b).

Difference $=\mid($ Experiments average max imum stress - Simulations $) /$ Experiments average max imum stress $\mid \%$ 
The difference is calculated regarding the average of the tensile maximum stress reached during the experiments for each tested coupon and the maximum stress reached by the FE model during the simulations for each case (element size and step size).

Considering off-axis $5^{\circ}$ coupons under tensile load, Figure 74 shows $\sigma_{x x} v s$. $\varepsilon_{x x}$ for both experiments and computational simulation. In this case, the load is supported not only by the fibers, but also by the matrix. Since the load increases, the ply stresses in local orientation also increase until failure. The failure process in off-axis $5^{\circ}$ coupons is a mixed mode of fiber and matrix failure as shown in Figure 75. The failure process initiates in some fibers, which are under high stress, as verified during the experiments. This effect is simulated by the failure of some elements under high stress in the FE model. Then, the stress field is redistributed for the other finite elements. Due to high loads to be supported by fibers, when the fiber failure mechanisms occur, the matrix is not capable to support the loads and fails in a brittle way $\left(E_{D}>E_{D C}\right)$. 


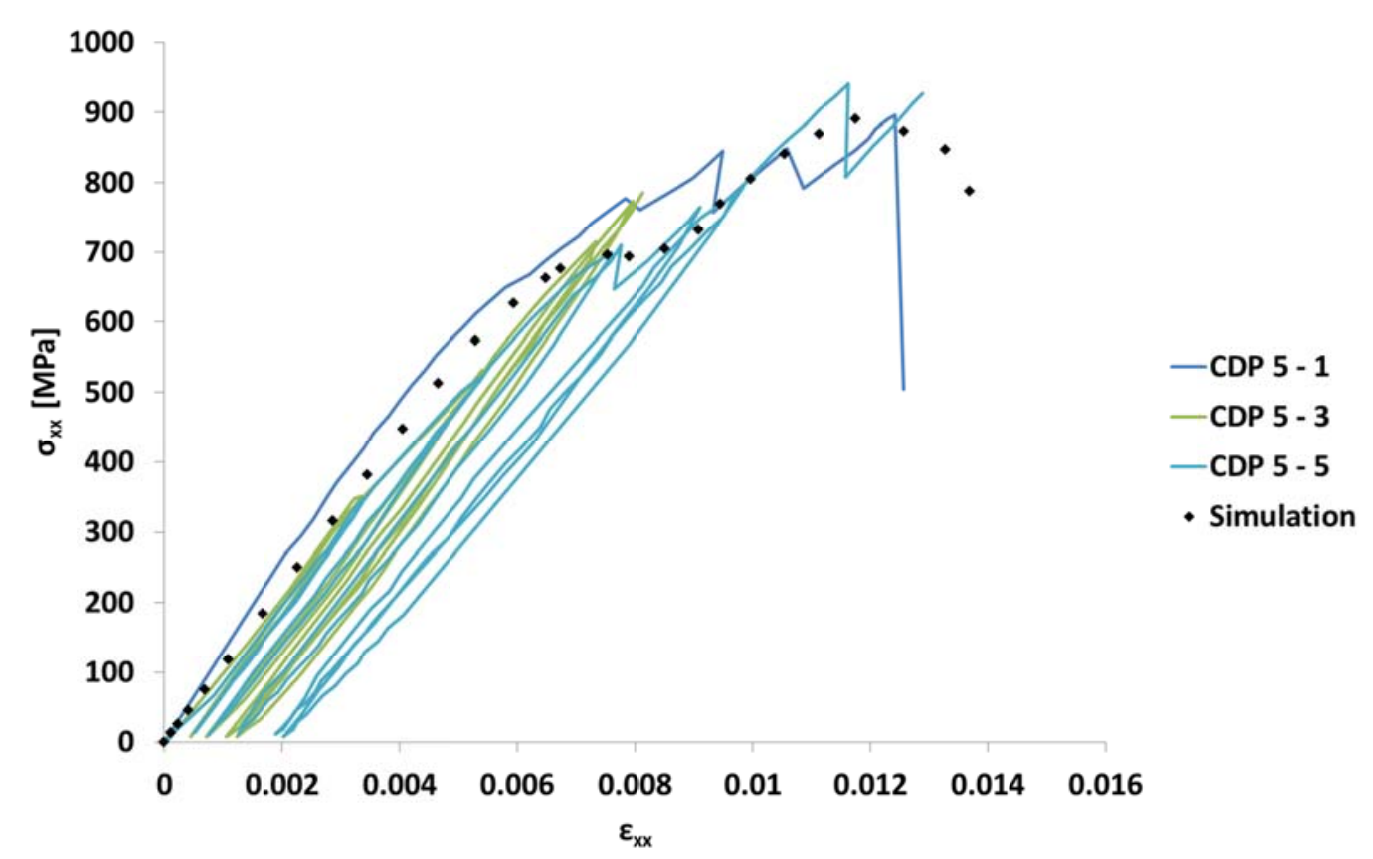

Figure 74: Stress vs. strain for tensile $\left[5^{\circ}\right]_{10}$ results - experimental vs. simulation.

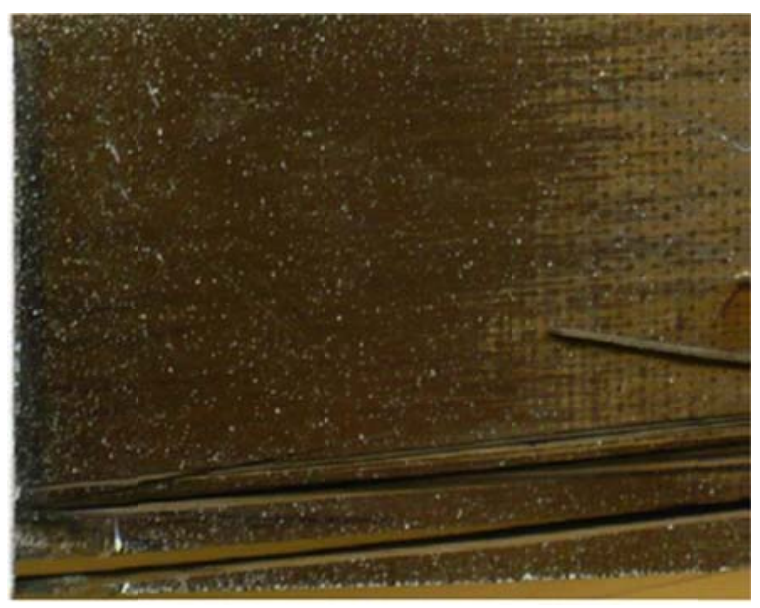

Figure 75 : Tensile $5^{\circ}$ coupon failure.

Table 40 summarises the experimental stresses and strains as well as the numerical results for tensile $\left[5^{\circ}\right]_{10}$ coupons. Regarding the average values, the relative difference among numerical simulation and experimental analyses is $1.9 \%$ for stress and6.8\% for strain at maximum stress. As observed, the error for the strains is higher than for stress due to nonlinear characteristic of the matrix failure model. 
Table 40: Tensile $\left[5^{\circ}\right]_{10}$ results for numerical and experimental analyses.

\begin{tabular}{ccc}
\hline Coupon & $\begin{array}{c}\text { Maximum stress } \\
{[\text { MPa] }}\end{array}$ & Strain at maximum stress \\
\hline CDP 5-1 & 896.64 & 0.01242 \\
\hline CDP 5-3 & 785.85 & 0.00850 \\
\hline CDP 5-5 & 940.41 & 0.01162 \\
\hline Experimental Average & 874.30 & 0.01085 \\
\hline Numerical Prediction & 890.90 & 0.01175 \\
\hline DIfference \% & 1.9 & 6.8 \\
\hline
\end{tabular}

Difference $=\mid($ Experiments average - Numerical Prediction $) /$ Experiments average $\mid \%$

\subsection{COMPRESSION RESULTS}

As previously mentioned, the compression behavior of composite materials is different from those for the tensile ones. In order to verify the model capacity to simulate the failure under compression, numerical results have been compared to experimental compression tests for off-axis $15^{\circ}$ and $30^{\circ}$ coupons. Also, as commented for off-axis tensile tests, the off-axis compression tests yield a biaxial stress state with normal stress $\left(\sigma_{x x}\right)$ and shear stress $\left(\tau_{x y}\right)$ fields.

The sensitivity studies are shown in Figure 76. The step size had strong influence on the model performance rather than on the element size. Figure 76(b) shows that for maximum step size greater than 0.03 ( $3 \%$ of the prescribed displacement), there is no difference in the Error for step size greater than 0.03 due to the convergence issues. In this case, the FE model with combination of $1.0 \mathrm{~mm}$ element size and 0.01 maximum for step size (i.e. $1 \%$ of the prescribed displacement for each increment) showed better results.

In the compression tests, the differences are too high in most of the cases (higher than $10 \%$ ) and the step size analysis had shown a huge influence for the FE model accuracy (Figure 76 (b)). Besides, if the amount of the maximum prescribed displacement decreases from $1 \%$ to $0.5 \%$ applied to each increment, the model accuracy will improve significantly. 


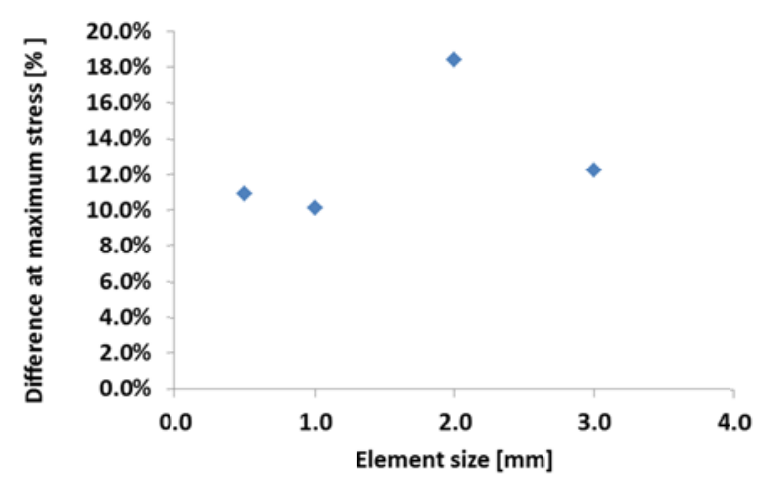

(a)

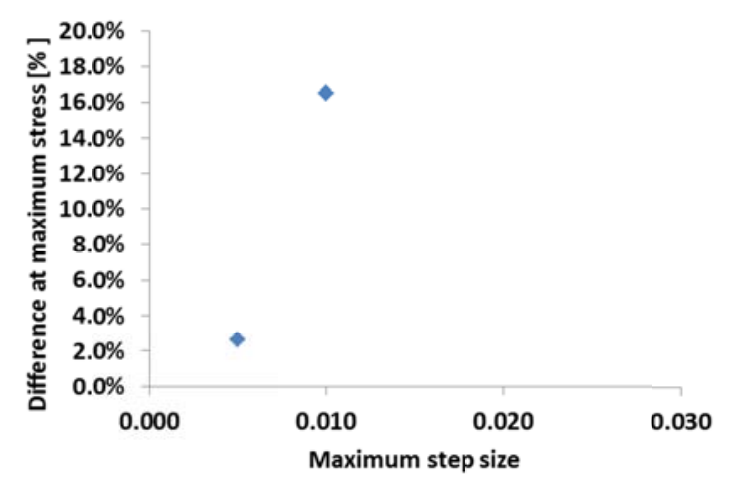

(b)

Figure 76: Compression $15^{\circ}$ coupons - finite element mesh sensitivity analysis (a) and step size analysis (b)

Difference $=\mid($ Experiments average min imum stress - Simulations $) /$ Experiments average $\min$ imum stress $\mid \%$

The difference is calculated regarding the average of the tensile maximum stress reached during the experiments for each tested coupon and the maximum stress reached by the FE model during the simulations for each case (element size and step size).

Figure 77 shows $\sigma_{x x} v s . \varepsilon_{x x}$ experimental test and the computational results for off-axis $15^{\circ}$ coupons under compression loading.

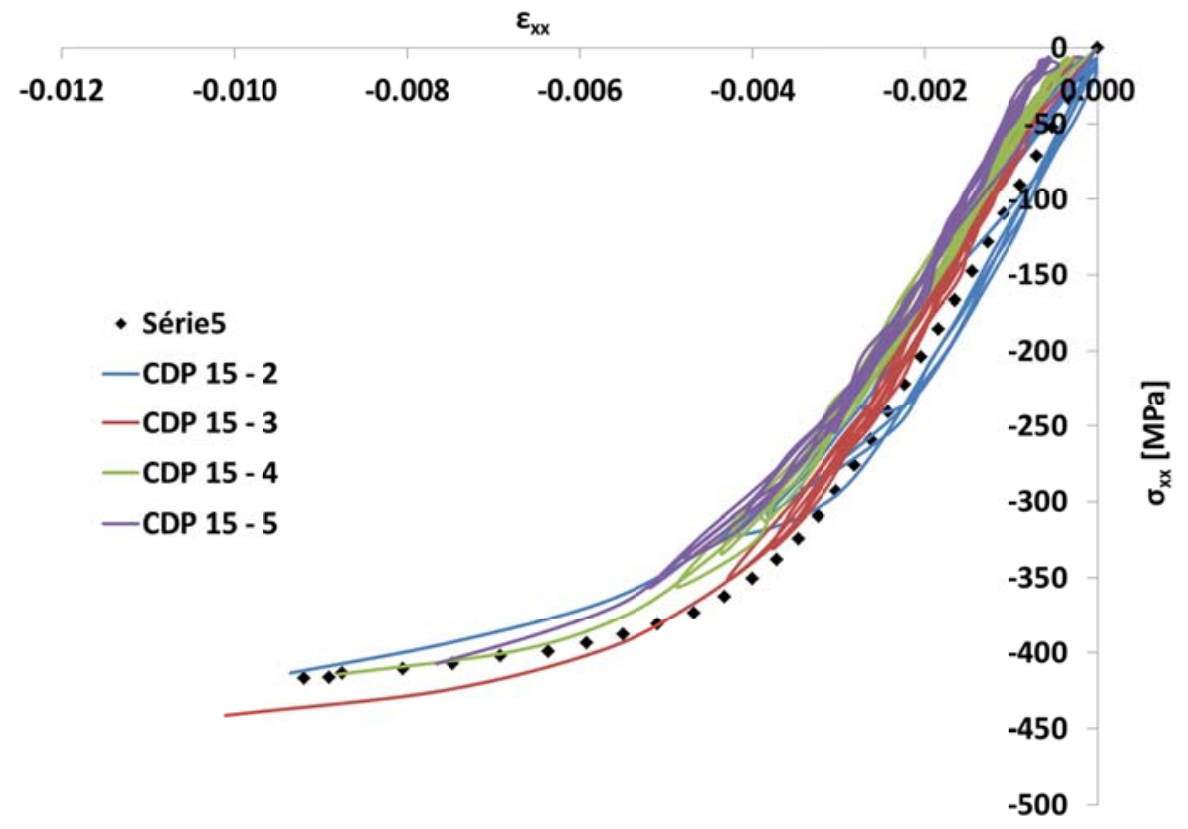

Figure 77: Stress vs. strain for compression $\left[15^{\circ}\right]_{10}$ results - experimental vs. simulation. 
In this case, similar to $5^{\circ}$ off-axis coupons under tension, the fibers support most of the load. However the fiber failure process is more complex than that in $5^{\circ}$ off-axis coupons under tension, due to fiber kinking and micro-buckling, which leads to lower failure loads; so the failure process is different as shown in Figure 78.

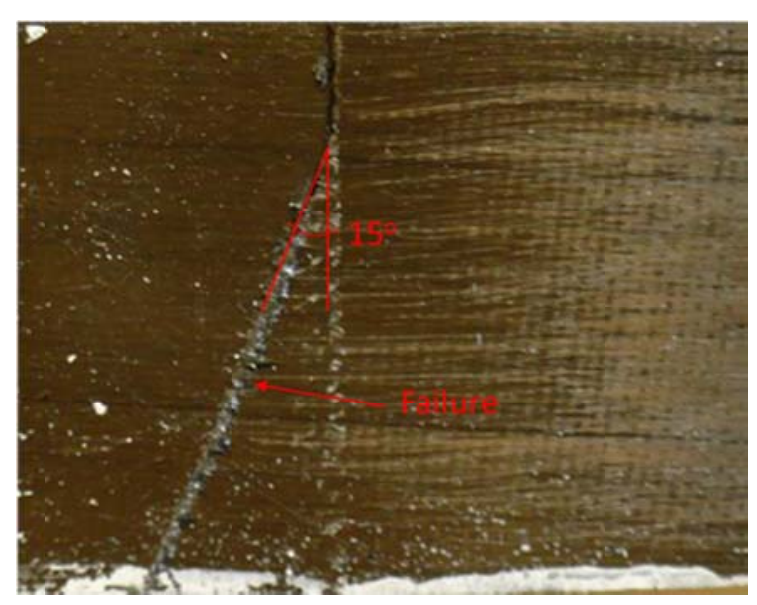

Figure 78: Compression $15^{\circ}$ coupon failure.

Table 41 summarizes the experimental results as well as the numerical results. In this case, the maximum error for stress was equal to $1.7 \%$ and, for the strain at maximum stress, it was equal to $2.2 \%$.

Table 41: Compression $\left[15^{\circ}\right]_{10}$ results for numerical and experimental analyses.

\begin{tabular}{ccc}
\hline Coupon & $\begin{array}{c}\text { Maximum stress } \\
{[\text { MPa] }}\end{array}$ & Strain at maximum stress \\
\hline CDP 15-2 & -393.32 & -0.0093 \\
\hline CDP 15-3 & -424.86 & -0.0101 \\
\hline CDP 15-4 & -414.33 & -0.0088 \\
\hline CDP 15-5 & -407.33 & -0.0077 \\
\hline Experimental Average & -409.96 & -0.0090 \\
\hline Numerical Prediction & -417.02 & -0.0092 \\
\hline Difference \% & 1.7 & 2.2 \\
\hline
\end{tabular}

Difference $=\mid($ Experiments average - Numerical Prediction $) /$ Experiments average $\mid \%$

Figure 79 shows the study for mesh convergence as well as for the maximum step size limit. As expected, the FE model with $0.5 \mathrm{~mm}$ element size and 0.005 for maximum step size (i.e. $0.5 \%$ of the prescribed displacement for each increment) showed better results than other models. 


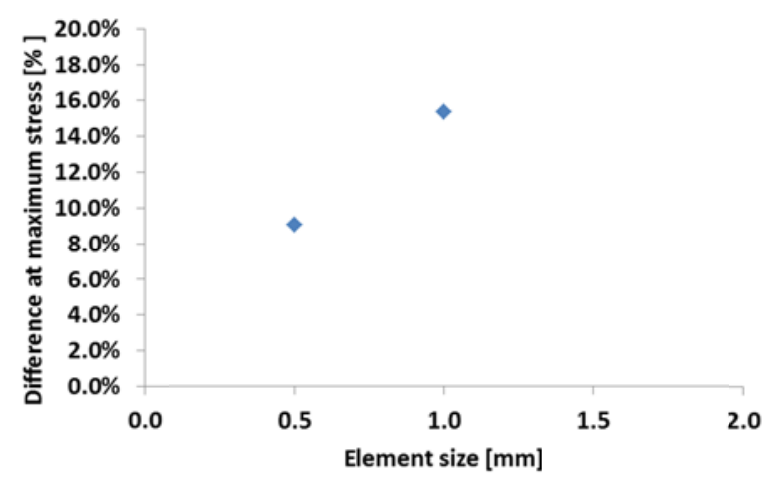

(a)

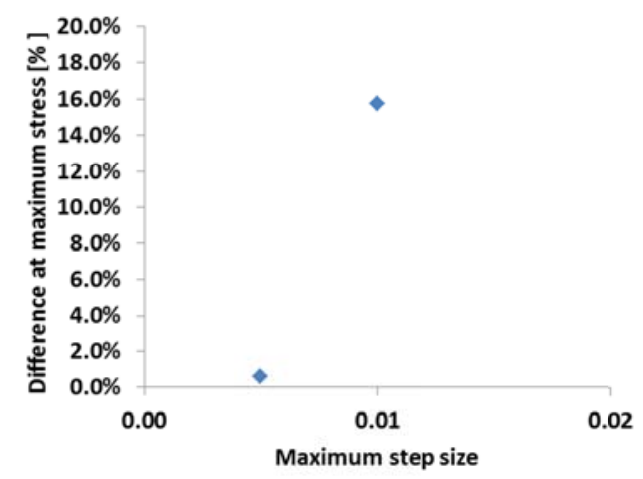

(b)

Figure 79: Compression $30^{\circ}$ coupons - finite element (a) mesh sensitivity analysis and (b) step size analysis

Difference $=\mid($ Experiments average $\min$ imum stress - Simulations $) /$ Experiments average $\min$ imum stress $\mid \%$

The difference is calculated regarding the average of the tensile maximum stress reached during the experiments for each tested coupon and the maximum stress reached by the FE model during the simulations for each case (element size and step size).

The off-axis $30^{\circ}$ coupons compression results in experiments and numerical simulation are shown in Figure 80. In this case, fibers still support more load than the matrix, but the difference between the load supported by fibers and the load supported by matrix is lower than the difference obtained by $\left[5^{\circ}\right]_{10}$ and $\left[15^{\circ}\right]_{10}$. 


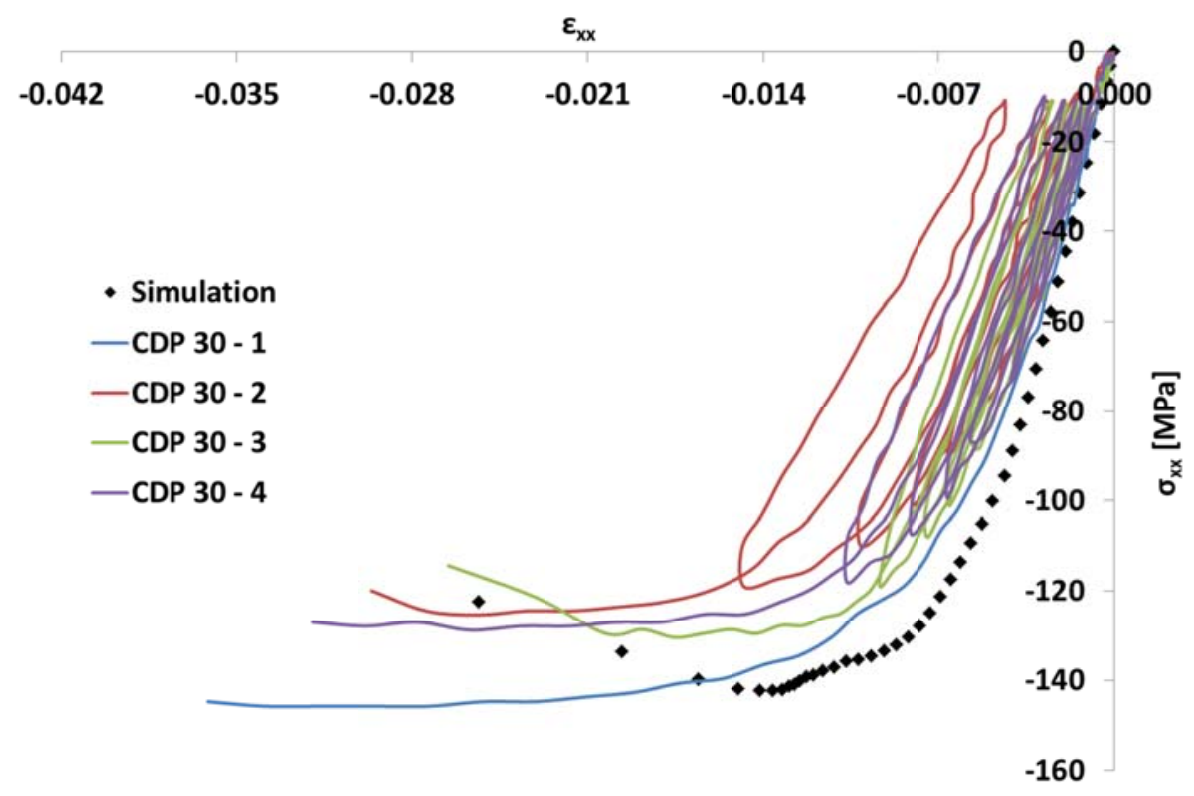

Figure 80: Stress vs. strain for compression $\left[30^{\circ}\right]_{10}$ results - experimental vs. simulation.

Table 42 summarizes the results for $30^{\circ}$ coupons under compression. In this case, the model has a reasonable performance to predict the maximum stress, but the error for strain at maximum stress is rather high. As previously mentioned, the $30^{\circ}$ coupons under compression fail due to buckling and the model presents a softening after maximum stress peak.

Table 42: Compression $\left[30^{\circ}\right]_{10}$ results for numerical and experimental analyses.

\begin{tabular}{ccc}
\hline Coupon & $\begin{array}{c}\text { Maximum Stress } \\
{[\mathbf{M P a}]}\end{array}$ & Strain at maximum stress \\
\hline CDP 30-1 & -145.80 & -0.0362 \\
\hline CDP 30-2 & -127.90 & -0.0254 \\
\hline CDP 30-3 & -130.40 & -0.0143 \\
\hline CDP 30-4 & -125.20 & -0.0257 \\
\hline Experimental Average & -132.33 & -0.0254 \\
\hline Numerical Prediction & -142.49 & -0.014 \\
\hline Difference \% & 7.7 & 44.9 \\
\hline
\end{tabular}

Difference $=\mid($ Experiments average - Numerical Prediction $) /$ Experiments average $\mid \%$

Despite the differences of load supported by each phase (fiber and matrix), the coupons geometry also affects the failure process. In the case of $30^{\circ}$ coupons, there are no fibers crossing all the gage length between the test machine grips. Since the matrix load carrying capacity is lower than the fibers, the failure load for $30^{\circ}$ coupons is lower than for $15^{\circ}$. Thus, the coupon failure is governed by the matrix. 
It is important to mention that the $30^{\circ}$ off-axis coupons compression test did not only fail due to "pure" compressive loads, but also due to buckling, mostly in the end of the test (under relative high loads). On the other hand, in off-axis $15^{\circ}$ coupons, buckling has not been detected until being close to the failure. Thus, the buckling phenomenon could explain the differences observed after the failure of $30^{\circ}$ off-axis coupons.

\subsection{Four-Point Bending Results}

Despite the simplicity of FE models used for both tension and compression tests, the FE model for four point bending is more complex. In this case, it is required to use contact algorithm (Figure 81) between the coupon and the test device, due to non-zero terms in the bending-stretching coupling matrix. Thus, it is not possible to make a simpler model without contact algorithm.

As for the simulation of tensile tests, 4-node fully integrated shell elements (S4) were used again to model the "flat" filament wound laminate. Each one of the ten layers was $0.2 \mathrm{~mm}$ thick with three integration points (top, medium and bottom point) for each layer analyse. Also the material model has been used for each layer calculations.

The parts of the four-point-bending device in contact with the coupon have been modelled by analytical rigid surfaces (represented by the blue cylinders in Figure 81, whose radius was $5.0 \mathrm{~mm}$ ). The boundary conditions have been applied at the cylinder reference points (RP). For the inner cylinders, prescribed displacement in $\mathrm{z}$ direction (blue arrow) has been applied and the other displacements $(\mathrm{Ux}=\mathrm{Uy}=0)$ and rotations around $\mathrm{x}$ and $\mathrm{z}$ axis $(\mathrm{Rx}$ and $\mathrm{Rz})$ were restricted at their respective reference points - RP (Figure 81). For the outer cylinders, all the displacements ( $\mathrm{Ux}, \mathrm{Uy}$ and $\mathrm{Uz}$ ) and rotations around $\mathrm{x}$ and $\mathrm{z}$ axis ( $\mathrm{Rx}$ and $\mathrm{Rz}$ ) were also restricted at their respective reference points. The contact interactions between the analytical rigid surfaces (blue cylinders) and flat filament wound coupon (shell elements) have been modelled by using Hard Contact for simulating the normal interactions and Penalty Method (with friction coefficient of 0.1 ) for modelling of the tangential interactions. In fact, there would be no influence on the simulations results, if the friction coefficient were changed to 0.3. It is important to highlight that the solver performs the analysis with the geometric nonlinear modulus deactivated. Otherwise, the numerical simulations would diverge from the experimental results, showing a stiffer behavior. Also, the small coupon curvature has not been considered in the FE analyses. 


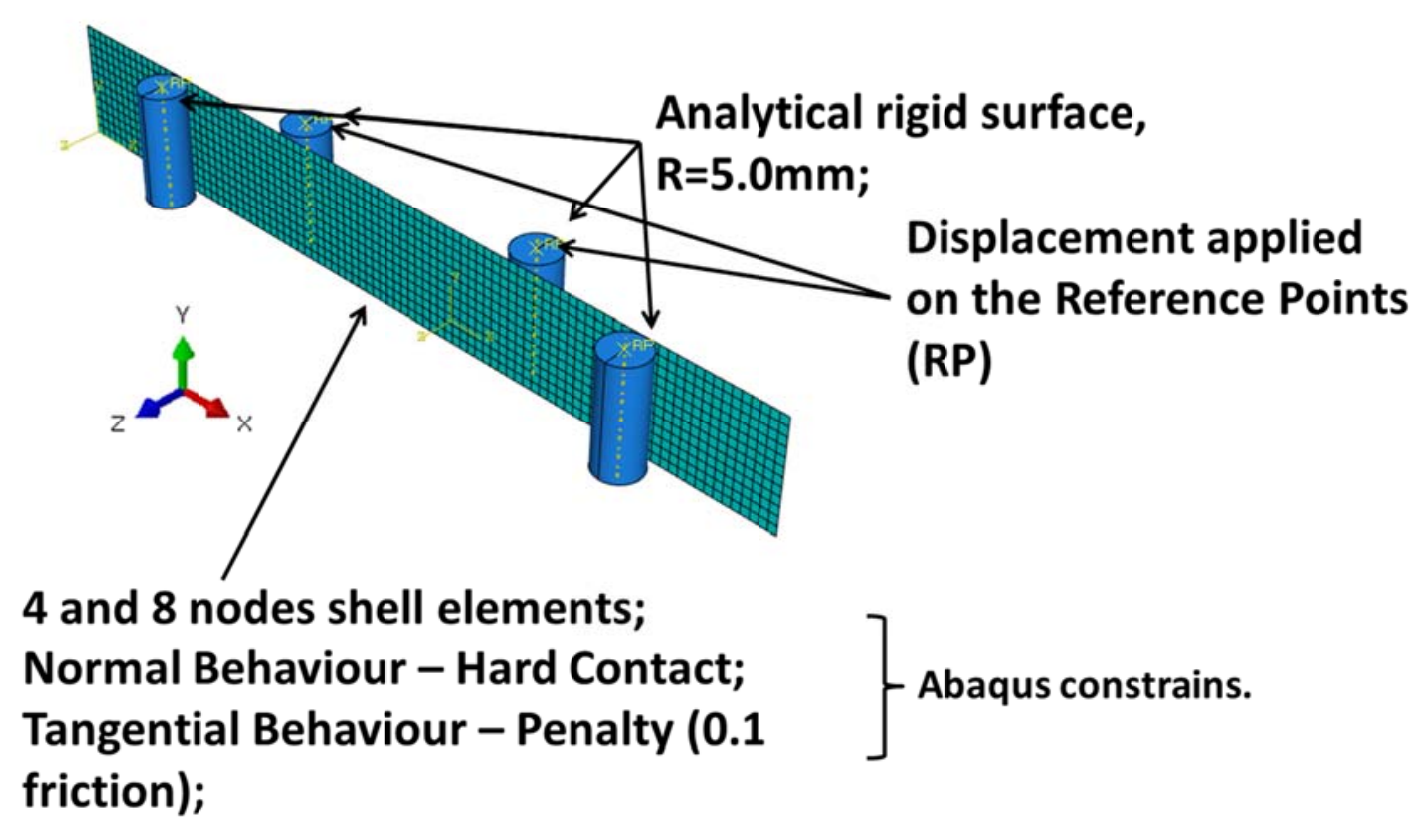

Figure 81: Four-point-bending finite element model ( $30^{\circ}$ coupons).

The step sensitivity and the mesh density studies are shown in Figure 82. The study of element size shows that, considering the element size, the predictions for maximum force are more sensible than the displacement at maximum load. On the other hand, the prediction for the displacement at maximum force is more affected by the step size. The maximum force has a reasonable error from 0.05 for maximum step size (i.e. $5 \%$ of the prescribed displacement). The FE model with the combination of $0.5 \mathrm{~mm}$ element size and 0.01 for maximum step size has shown a better result.

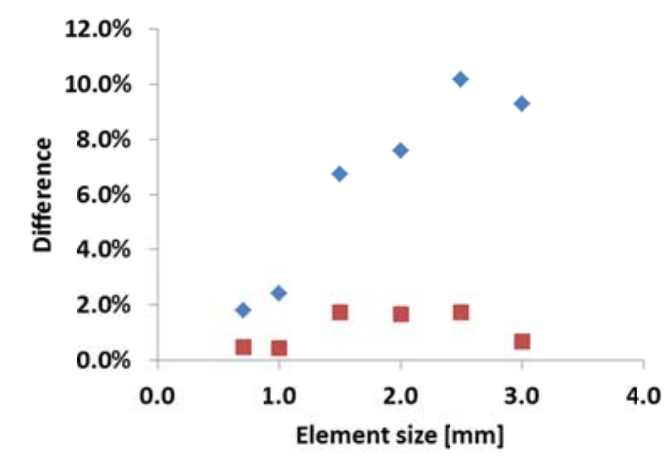

(a)

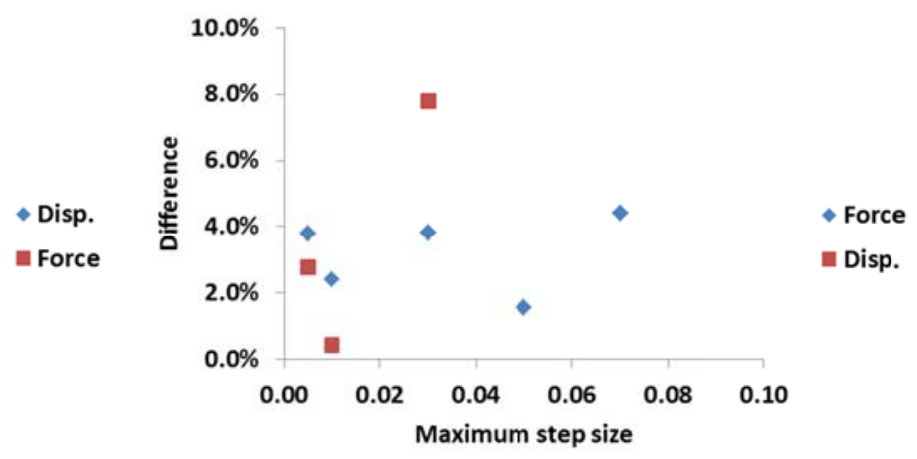

(b)

Figure 82: Off-axis $30^{\circ}$ coupons 4-Point bending - finite element mesh convergence analysis (a) and step size analysis (b). Difference $=\mid($ Experiments average - Simulations $) /$ Experiments average $\mid \%$ 
Figure 83 shows the simulation and experimental results for the off-axis $\left[30^{\circ}\right]_{10}$ four-point bending tests. The experimental analyses showed a good correlation among the coupons. Only two coupons, CDP 30-3 and CDP 30-7, showed some damage at high load levels before rupture. For the other coupons, no significant damage or delamination has been observed prior to collapse. Since the stresses discontinuity has been lower between layers with the same orientation, delaminations for off-axis laminates have been more unlikely than for laminates with different ply orientation (Herakovich, 1998). Therefore, this is the reason for the proposed material model (with only intra-ply damage formulation) to show a good convergence to the experimental data.

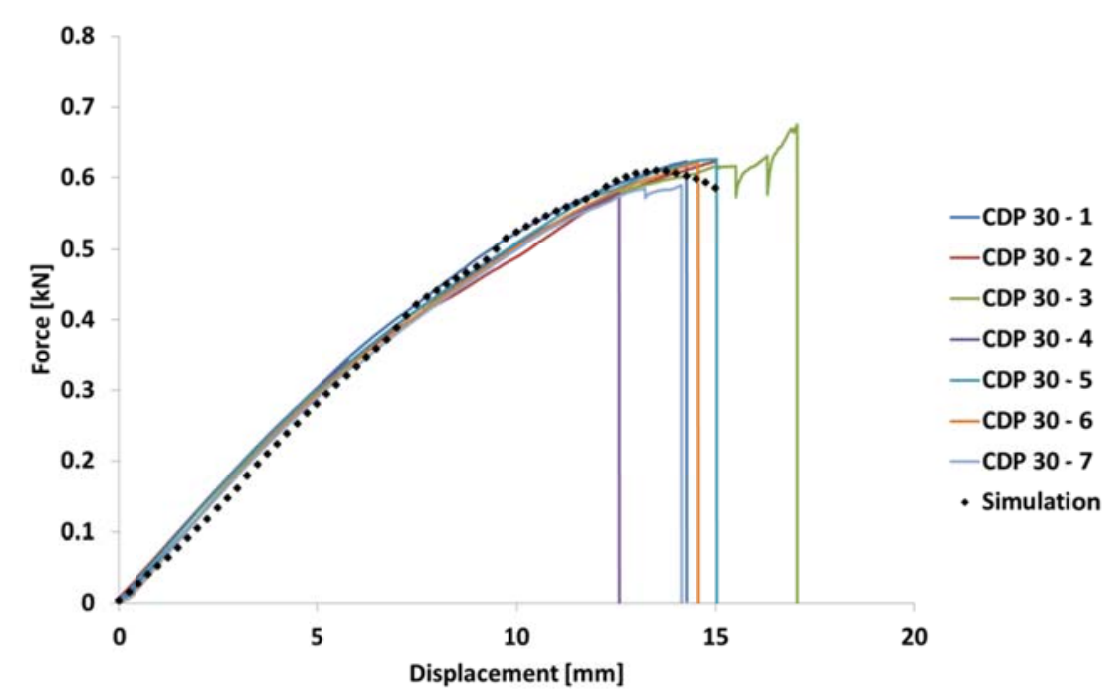

Figure 83: Force vs. Displacement for four- point bending $\left[30^{\circ}\right]_{10}$ results - numerical and experimental analyses.

Table 43 summarises the experimental and numerical results for four point bending $\left[30^{\circ}\right]_{10}$ coupons. The relative difference between the average experimental results and numerical prediction is $1.6 \%$ for maximum force and $2.3 \%$ for displacement. 
Table 43: $\left[30^{\circ}\right]_{10}$ four point bending for numerical and experimental analyses.

\begin{tabular}{ccc}
\hline Coupon & Maximum Force [kN] & $\begin{array}{c}\text { Displacement at Maximum } \\
\text { force [mm] }\end{array}$ \\
\hline CDP 30-1 & 0.62 & 14.25 \\
\hline CDP 30-2 & 0.62 & 15.00 \\
\hline CDP 30-3 & 0.68 & 17.05 \\
\hline CDP 30-4 & 0.58 & 12.57 \\
\hline CDP 30-5 & 0.63 & 15.03 \\
\hline CDP 30-6 & 0.62 & 14.56 \\
\hline CDP 30-7 & 0.59 & 14.13 \\
\hline Experiments Average & 0.62 & 14.66 \\
\hline Numerical Prediction & 0.61 & 15.00 \\
\hline Difference \% & 1.61 & 2.32 \\
\hline
\end{tabular}

Difference $=\mid($ Experiments average - Numerical Prediction $) /$ Experiments average $\mid \%$

As mentioned before, the off-axis coupons exhibit bending-stretching coupling. The coupons warp during the experiments and this phenomenon is very well predicted by the finite element model as shown in Figure 84. The simulations had shown a $1.12 \mathrm{~mm}$ maximum gap between the four point bending device and coupon where the cylinders did not touch the coupon.

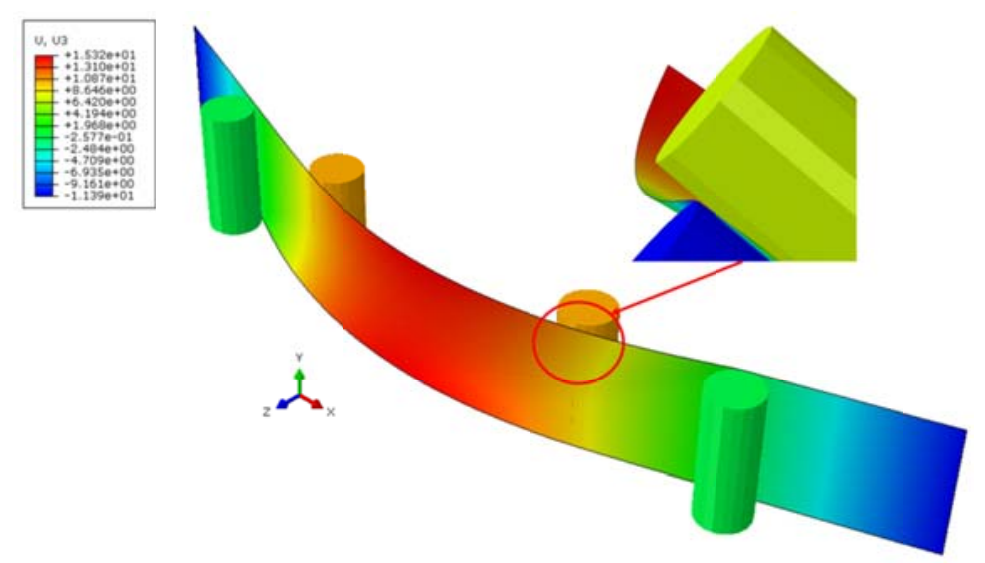

Figure 84: Displacement field in z direction (blue arrow) and the bending-stretching coupling effect between the support and the coupon (in detail).

Figure 85 shows the stress fields $\left(\sigma_{11}, \sigma_{22}\right.$ and $\left.\tau_{12}\right)$ predicted by the FE model, just before the complete failure of the laminate for both the most tensile loaded layer $\left(10^{\text {th }}\right.$ layer $)$ and the 
most compression loaded layer $\left(1^{\text {st }}\right.$ layer$)$. Some stress concentrations have been observed in some parts of the laminate as shown by Figure 85 . This can be verified through the difference of the stresses colour representation for the external layers where the cylinders touch the coupon. Despite the localisation issue, the numerical model predicts very well the laminate behavior for this load case. Moreover, during the experiments, there was no matrix crushing close to the contact areas between the coupons and the device supports. The failure always occurs inside the span region, which is between the inner cylinders, and the FE analysis predicts this phenomenon well. Also, the damage model shows that the failure mode is dominated by the matrix as observed in the experiments.

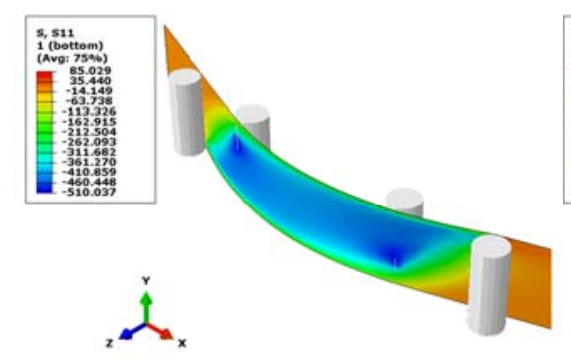

(a)

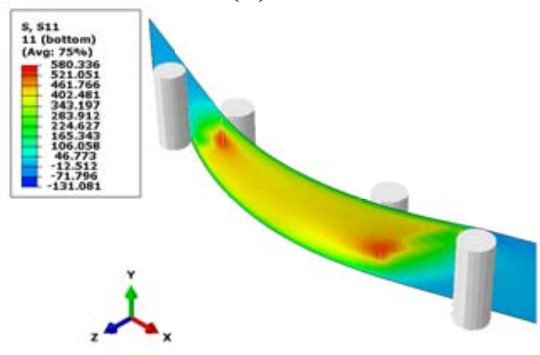

(d)

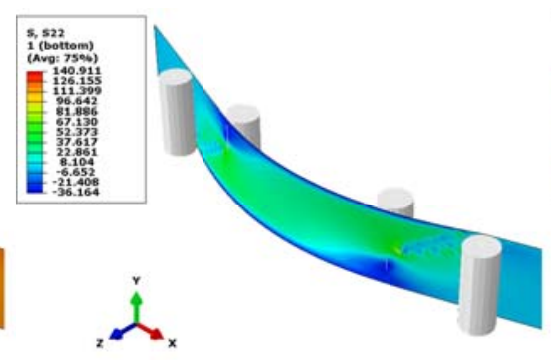

(b)

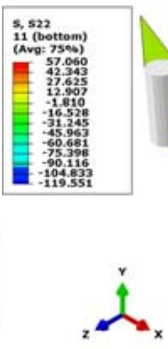

(e)

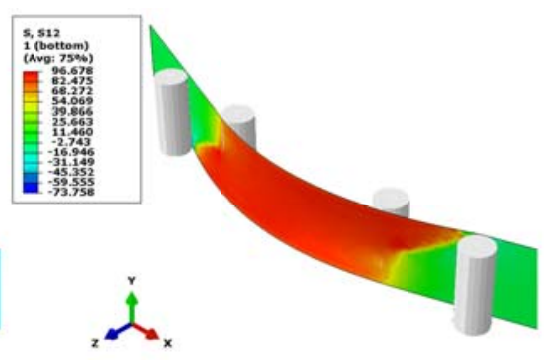

(c)

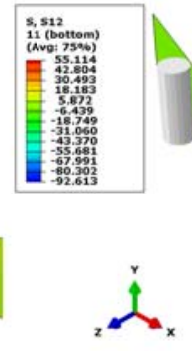

(f)

Figure 85: Stress fields just before the complete failure of the laminate: (a) Layer $1 \sigma_{11}$; (b) Layer 1 $\sigma_{22} ;$ (c) Layer $1 \tau_{12}$; (d) Layer $10 \sigma_{11}$; (b) Layer $10 \sigma_{22}$ and (c) Layer $10 \tau_{12}$.

\subsection{Conclusions For QUASI-STATIC Tests AND Simulations}

The proposed damage model has been implemented as a user material subroutine UMAT, which can very well predict the behavior of quasi-flat filament wound composite laminates not only under tensile and compression loading, but also under four-point- bending conditions. In the latter case, the difference between numerical and average experimental 
results is only $1.61 \%$ at maximum force. Therefore, it is concluded that the material model can be applied to simulate accurately intra-ply failure of flat filament wound composite laminates. It can be used to assist the design of composite structures made by filament winding process. Another important advantage is that the model parameters are not so complicated to be identified. This characteristic makes this model very attractive to be applied to the industrial environment. However, the damage model cannot predict delaminations yet.

\subsection{IMPACT ANALYSES}

This part shows the material model applied to simulate impact on filament winding cylinders. Since the $31 \mathrm{~J}$ impact showed more damage, all the simulations and analyses have been performed at this impact energy level.

Several numerical tests have been carried out to verify the influence of mesh density, element type (quadrilateral or triangular), contact algorithm and damping effects. The results and discussions are presented in the next sections.

\subsubsection{MATHEMATiCAL FORMULATION}

The response of unidirectional carbon fiber composite for low velocity impact may be regarded as rate-independent. Thus, it is not necessary to modify the material model used in the previous chapter, what was applied to quasi-static simulations. However, it is important to highlight that specified user material subroutines, defined as VUMAT, have been developed to be linked to ABAQUS ${ }^{\mathrm{TM}} /$ explicit.

\subsubsection{Finite ELEMENT MODELS}

The impact tests on composite cylinders have been simulated by using ABAQUS $^{\mathrm{TM}} /$ explicit. The finite element model boundary conditions are shown in Figure 86(a). In the cylinder bottom, the $z$ direction nodes displacements are restricted $\left(U_{Z}=0\right)$ to simulate the contact with the V-block (Figure 43 and Figure 86(a)). In the impactor head, the displacements in $\mathrm{x}\left(\mathrm{U}_{\mathrm{x}}\right)$ and $\mathrm{y}\left(\mathrm{U}_{\mathrm{y}}\right)$ directions are restricted as well as the rotations about $\mathrm{x}$ 
$\left(\mathrm{R}_{\mathrm{x}}\right)$, y $\left(\mathrm{R}_{\mathrm{y}}\right)$ and $\mathrm{z}\left(\mathrm{R}_{\mathrm{z}}\right)$ axis. The impactor set according to its center line is normal for the cylinder top plane. Moreover, the impactor hits the cylinder in the middle of a straight line, which belongs to the cylinder top plane. The initial distance between the impactor vertex and the contact point is $2.0 \mathrm{~mm}$.

Figure 86 (b) shows the impactor head details. Its radius has $8.0 \mathrm{~mm}$ and a point of its mass is equal to $3.24 \mathrm{~kg}$, which is used to account the impactor frame and additional masses. The impactor head has been modeled by using rigid triangular elements (defined as R3D3). Besides, a reference point in the impactor vertex has been created to calculate the reaction forces.

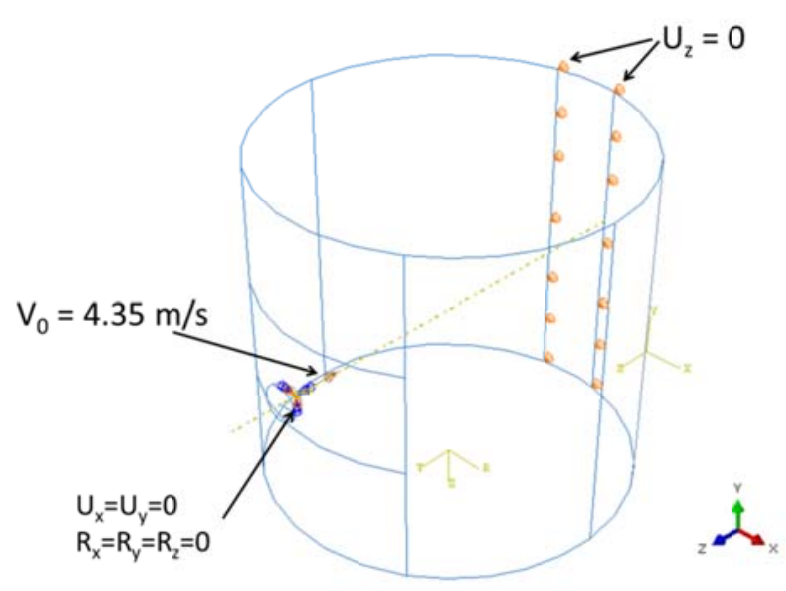

(a)

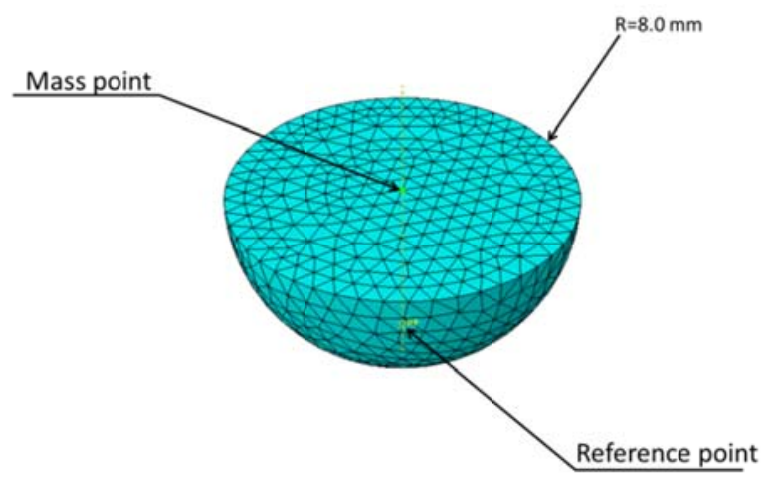

(b)

Figure 86: Finite element model: (a) Cylinder geometry, boundary conditions and initial conditions at $30 \mathrm{~J}$ impact energy; (b) Impactor geometry, reference point, mass point and mesh.

\subsubsection{Mesh sensitivity studies}

In order to study the mesh influence on impact simulations, three different mesh densities as well as two different elements types (triangular and regular quadrilateral) have been investigated. For some analyses, it was used the four-node reduced integration homogeneous shell element, which has six degrees of freedom per node (S4R) with three different structured meshes densities (Figure 87 (a), (b) and (c)). In other analyses, it was used a three node reduced integration homogeneous shell element, which has six degrees of freedom per node 
(defined as S3R) (Figure 87 (d)). In this case, a free mesh algorithm was applied. Only one mesh refinement was verified in this case, but the used mesh was not coarse.

Despite the element shape being equal to all cylinders, the laminate has been modeled considering the thickness values of each layer as specified in Table 14. The material properties were obtained by the experiments already described in the previous Chapter. Furthermore, the reduced integration elements have been used to improve the simulation time.

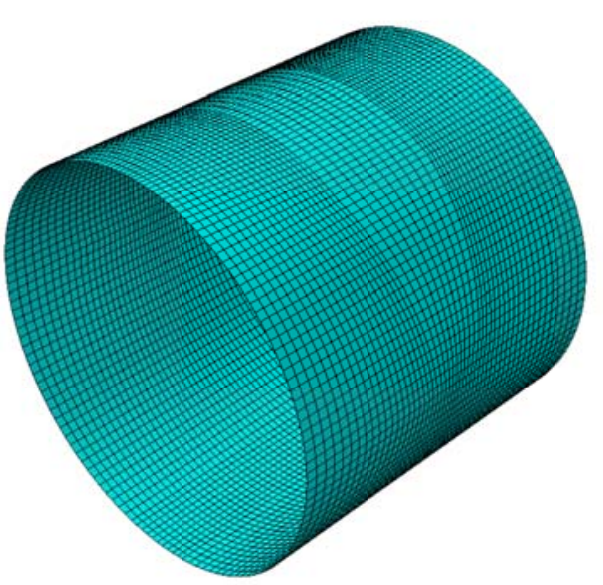

(a)

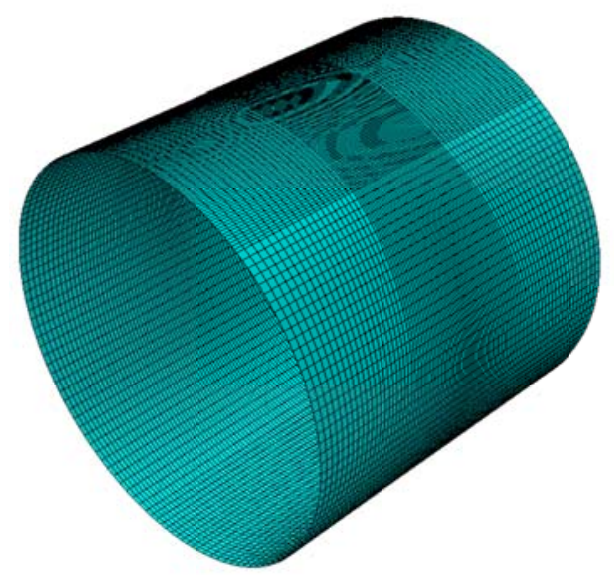

(c)

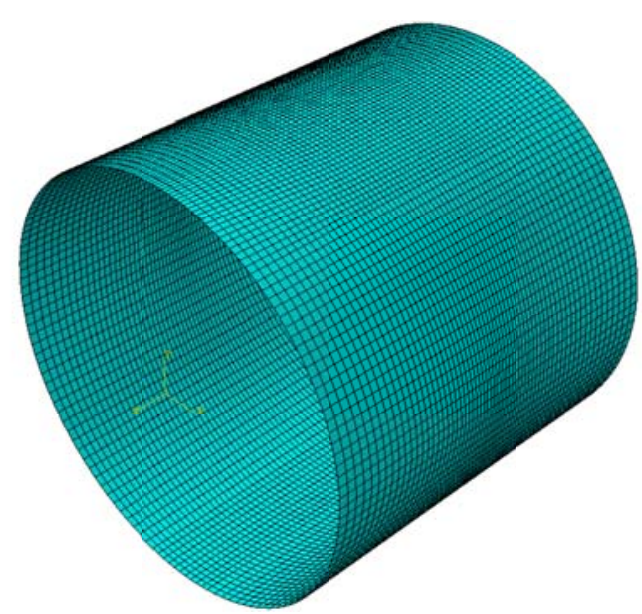

(b)

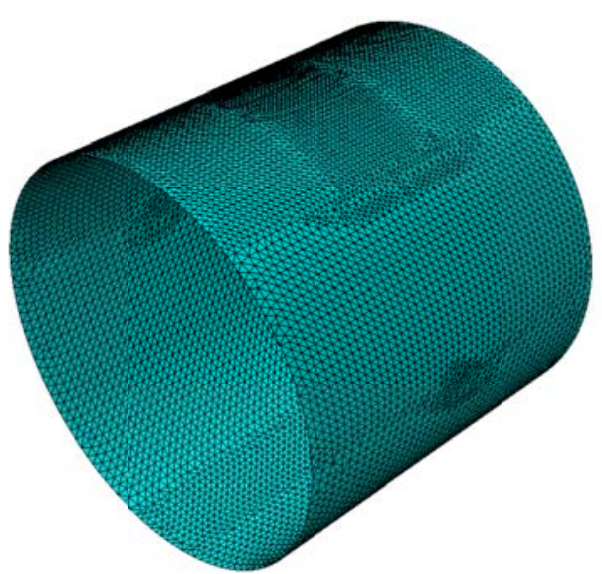

(d)

Figure 87: Meshes for FE models. Three different meshes density used with structured square elements: coarse mesh (a); intermediate refined mesh (b); the most refined mesh (c). Free mesh algorithm with triangular elements (d) to verify the element type influence.

In Figure 82(a), the mesh has 7350 elements and 7497 nodes, but in Figure 82(b), the mesh (intermediate refinement) has 9858 elements and 10017 nodes. And, in Figure 82(c), the 
mesh has 17716 elements and 17922 nodes. Finally, in Figure 82(d), the mesh has 16734 elements and 8514 nodes.

The simulations conditions (contact algorithm and boundary conditions) are the same for all meshes. The results for the mesh influence are shown in Figure 88 at type B cylinders, which show the reaction force of the impactor vs. time. As expected, the third mesh (the most refined mesh with quadrilateral elements) performed better results. The triangular element mesh showed the worst performance.

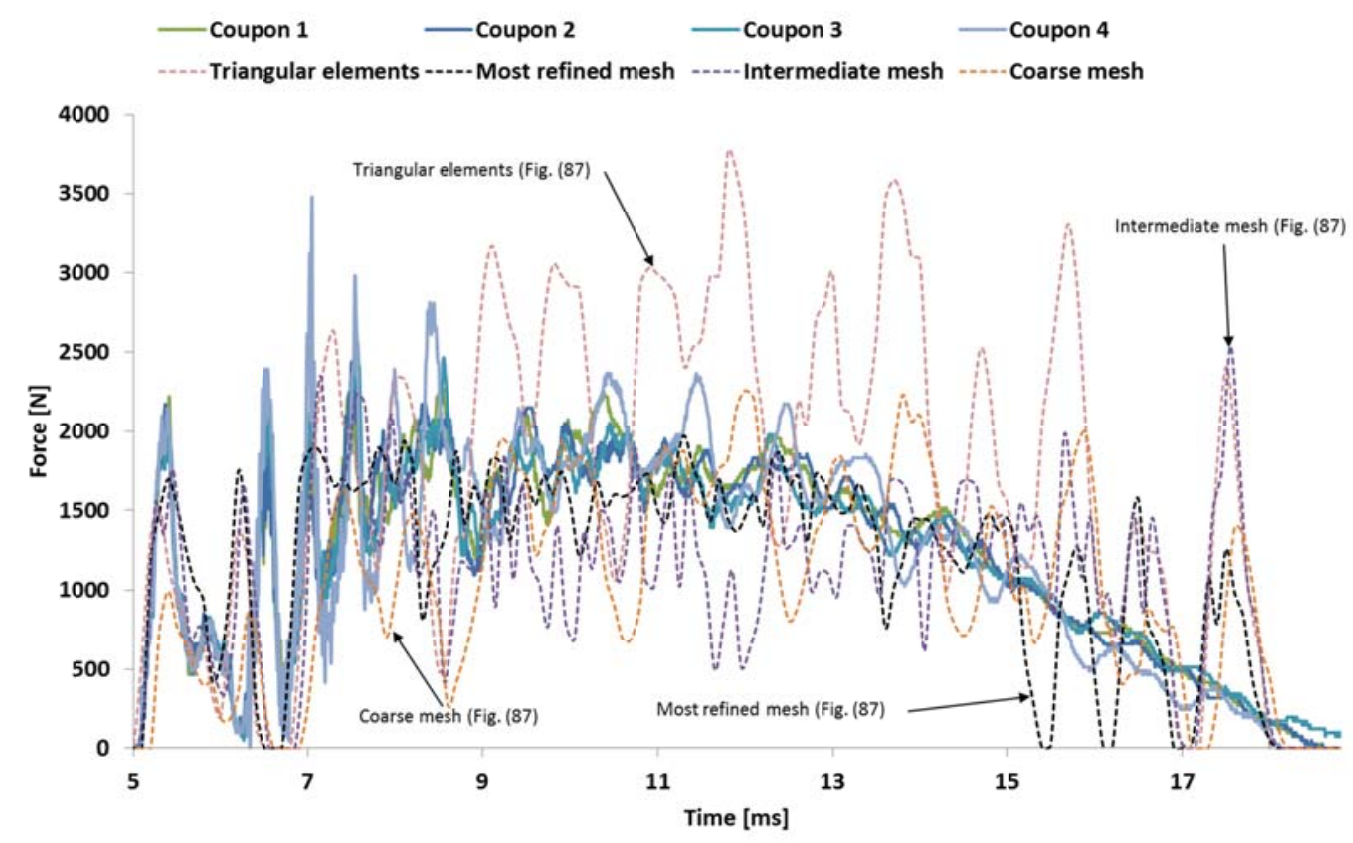

Figure 88: Effect of mesh density and element type (see Figure 87) for type B cylinders.

The criteria to choose the best simulation results consist on the comparison of the force oscillations frequency, the location of the force peaks and the visual observation of the curves (experiments and simulations).

The analyses of results for mesh sensitivity shown by type B cylinders can be extended for Type A and Type C cylinders.

\subsubsection{Contact algorithm studies}

After the mesh sensitivity study, the contact algorithm effect on the force response was also analyzed. To simulate the friction between the cylinder and impactor head, the contact tangential behavior has been modeled by using Penalty Method, which accounts the 
interaction friction (coefficient equal to 0.3 in this case). It is important to mention that the tangential behavior had no influence on the numerical response, once there was no slip between the impactor and cylinder in the FE model.

On the other hand, the normal contact behavior had a significant effect on the simulations responses as well as the selected contact algorithm (surface to surfacie or general contact Simulia (2010)). In order to verify this influence, 6 (six) different cases have been studied, i.e. one case for hard contact (Figure 89(a)) and 5 (five) cases for softening contact (exponential pressure-over closure relationship, Figure 89(b)). It is important to highlight that ABAQUS ${ }^{\mathrm{TM}}$ only allows using softening behavior for surface-surface contact algorithm.

According to the software manual (Dassault Systèmes Simulia Corp, 2010), the user can define the pressure value at zero over closure, $p^{0}$, and the initial contact distance, $c_{0}$ (Figure 89 (b)). The equations for this softening behavior are shown below.

$$
\begin{gathered}
p=0 \quad \text { for } h \leq-c_{0} \\
p=\frac{p^{0}}{e-1}\left[\left(\frac{h}{c_{0}}+1\right)\left(\exp \left(\frac{h}{c_{0}}+1\right)-1\right)\right] \quad \text { for } h>-c_{0} \\
\frac{d p}{d h}=0 \quad \text { for } h \leq-c_{0} \\
\frac{d p}{d h}=\frac{p^{0}}{e-1}\left[\frac{1}{c_{0}}\left(\frac{h}{c_{0}}+2\right) \exp \left(\frac{h}{c_{0}}+1\right)-\frac{1}{c_{0}}\right] \quad \text { for } h>-c_{0}
\end{gathered}
$$

Where $h$ is the overclosure, $p$ is the contact pressure. Figure 89 shows the relation between the clearance of the contact bodies and pressure.
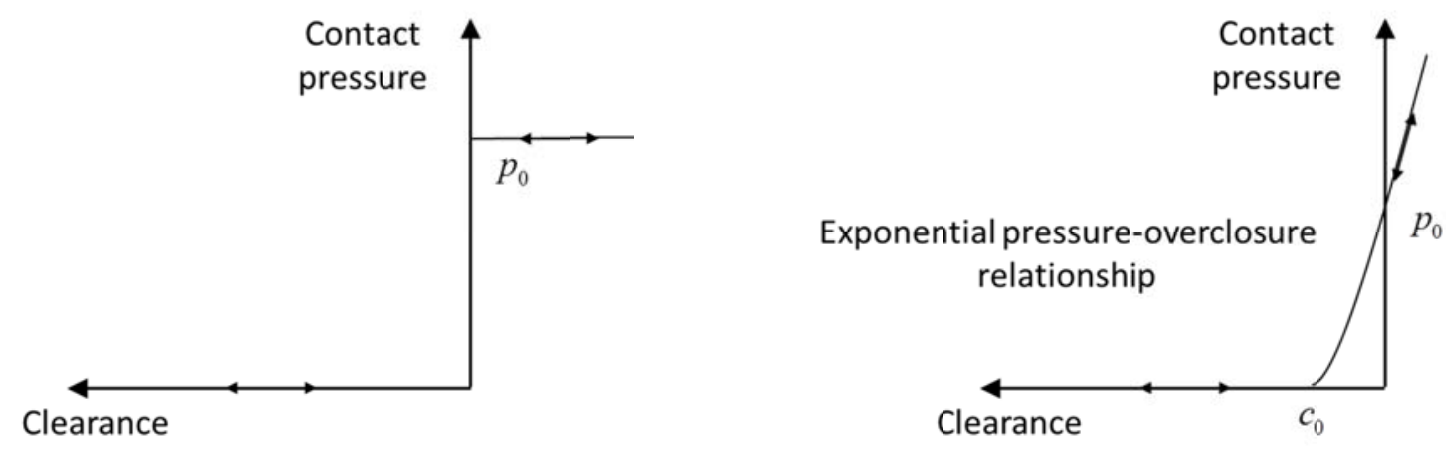
(a)

(b)

Figure 89: Hard contact (a) and exponential pressure-overclosure relationship (b).

The mesh presented at Figure 87(b) has been used for these analyses in order to keep the simulation time reasonable and the conclusions can be extended for the most refined mesh. The same boundary and initial conditions have been used for all the models. General contact has been used for hard contact to simulate normal behavior and surface-to-surface for softening behavior.

Table 44 summarizes the case studies for softening behavior. The results for the different contact properties are shown in Figure 90 for type A cylinders. This figure also shows the experimental results to assist the analysis of the contact influence on the numerical simulations.

Table 44: Contact parameters sensitivity study

\begin{tabular}{ccc}
\hline Case & $\begin{array}{c}p^{0} \\
{[\mathbf{P a}]}\end{array}$ & $\begin{array}{c}c_{0} \\
{[\mathbf{m}]}\end{array}$ \\
\hline $\mathrm{C} 1$ & $10^{11}$ & 0.0001 \\
& & \\
\hline $\mathrm{C} 2$ & $10^{15}$ & 0.0001 \\
\hline $\mathrm{C} 3$ & $10^{5}$ & 0.0001 \\
\hline $\mathrm{C} 4$ & $10^{11}$ & 0.001 \\
\hline $\mathrm{C} 5$ & $10^{11}$ & 0.00001
\end{tabular}

Observing the analysis in Figure 90, based on the initial part of the impact force history (from $4.5 \mathrm{~ms}$ to $6.5 \mathrm{~ms}$ ), it can be observed that all the contact laws provide good predictions. In fact, they start to diverge from the time around $6.5 \mathrm{~ms}$, for all FE models, which used the surface-to-surface contact algorithm (softening contact). Thus, they overestimated the next force peak. After that, this contact algorithm can smooth all the high frequencies oscillations of the force history. Moreover, the clearance factor $\left(c_{0}\right)$ showed to be more relevant than the initial pressure $\left(p^{0}\right)$. It is clear in Figure 90, where the softening contact did not provide good results, despite the combination of the parameters. 
On the other hand, the general contact algorithm with hard contact to simulate normal behavior has shown better predictions. The high frequencies force oscillations were simulated with a reasonable precision up to $12.5 \mathrm{~ms}$.

Despite good performance reached by general contact for impact simulation on cylinders, all ABAQUS ${ }^{\mathrm{TM}}$ contact algorithms fail to simulate correctly the final part of the impact (from $12.5 \mathrm{~ms})$.

To evaluate the FE model results, some variables can be compared to experimental results, e.g. the force peaks intensity, the time of the force peaks, frequency of oscillations and visual appearance of the FE results.

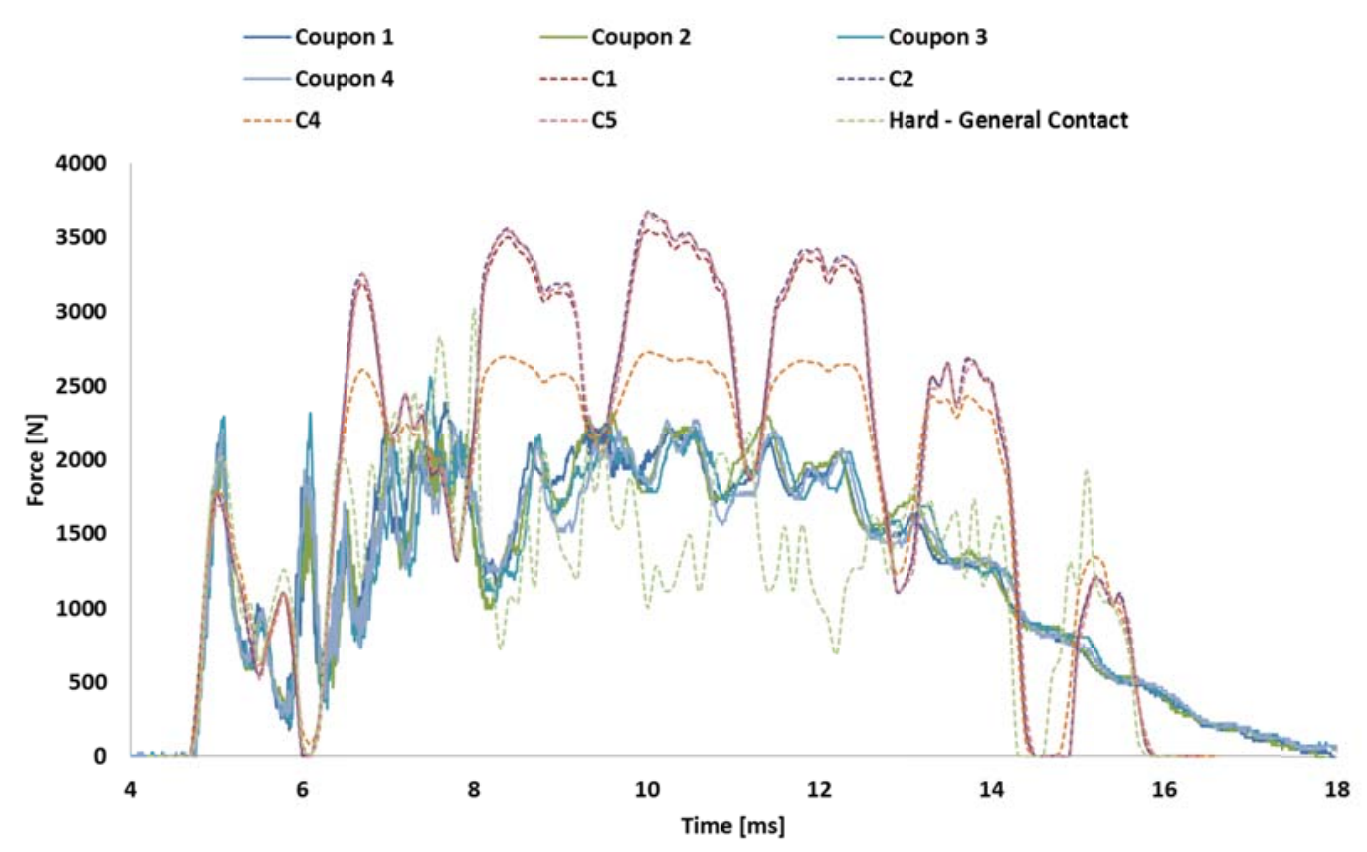

Figure 90: Type A contact parameters sensitivity study

It is possible to extend those conclusions for the other cylinders lay-ups (type B and C). Therefore, all impact simulations using ABAQUS ${ }^{\mathrm{TM}} /$ explicit must use the normal hard contact combined to general contact algorithm.

\subsubsection{Damping coefficient studies}

The dissipation of impact energy is another important issue for simulating the impact on composite filament winding cylinders. Part of the impact energy is dissipated by irreversible 
process as damage (matrix and fiber damage and delaminations), but the other part is dissipated by damping.

Composite materials damping is dependent on several factors, such as: fiber volume fraction, composite lay-up, environmental factors, force magnitude, etc. (Zabaras \& Pervez, 1990). Furthermore, the structure geometry has an important influence on the impact response.

As commented before, ABAQUS ${ }^{\mathrm{TM}}$ provides the Rayleigh's damping model for direct integration dynamic analysis to simulate energy dissipation through the damping phenomenon (Simulia, 2010). In fact, for finite element analysis, damping is treated as a matrix, which can be dealt with either as a material property or as a numerical object to oppose the excitation forces (Kyriazoglou \& Guild, 2007).

Since damping modifies the finite element model behavior, it is necessary to evaluate its influence on the impact force history. Several values for low frequency Rayleigh's parameter $(\alpha)$ have been analyzed by three different cylinders (Type A, Type B and Type C), once the damage mechanisms are different for each cylinder lay-up. The $\alpha$ values range from zero (no damping) up to unrealistic values in order to model correctly the final part of the impact event (e.g. 1800).

The stiffness proportional parameters at high frequency $(\beta)$ for all cylinders were set to "zero" in order to obtain the suitable fit between experimental and numerical results. This parameter makes the high frequency force oscillations smoother. Also, in order to avoid the increase of the simulation time, this parameter was set to "zero", because the stable time integration for explicit simulations could be affected (Dassault Systèmes Simulia Corp, 2010).

All the studies presented forward have used the same mesh density and normal hard contact behavior combined to general contact algorithm.

\subsection{Type A damping effect.}

Figure 91 shows the results for type A cylinders impactor force history. 


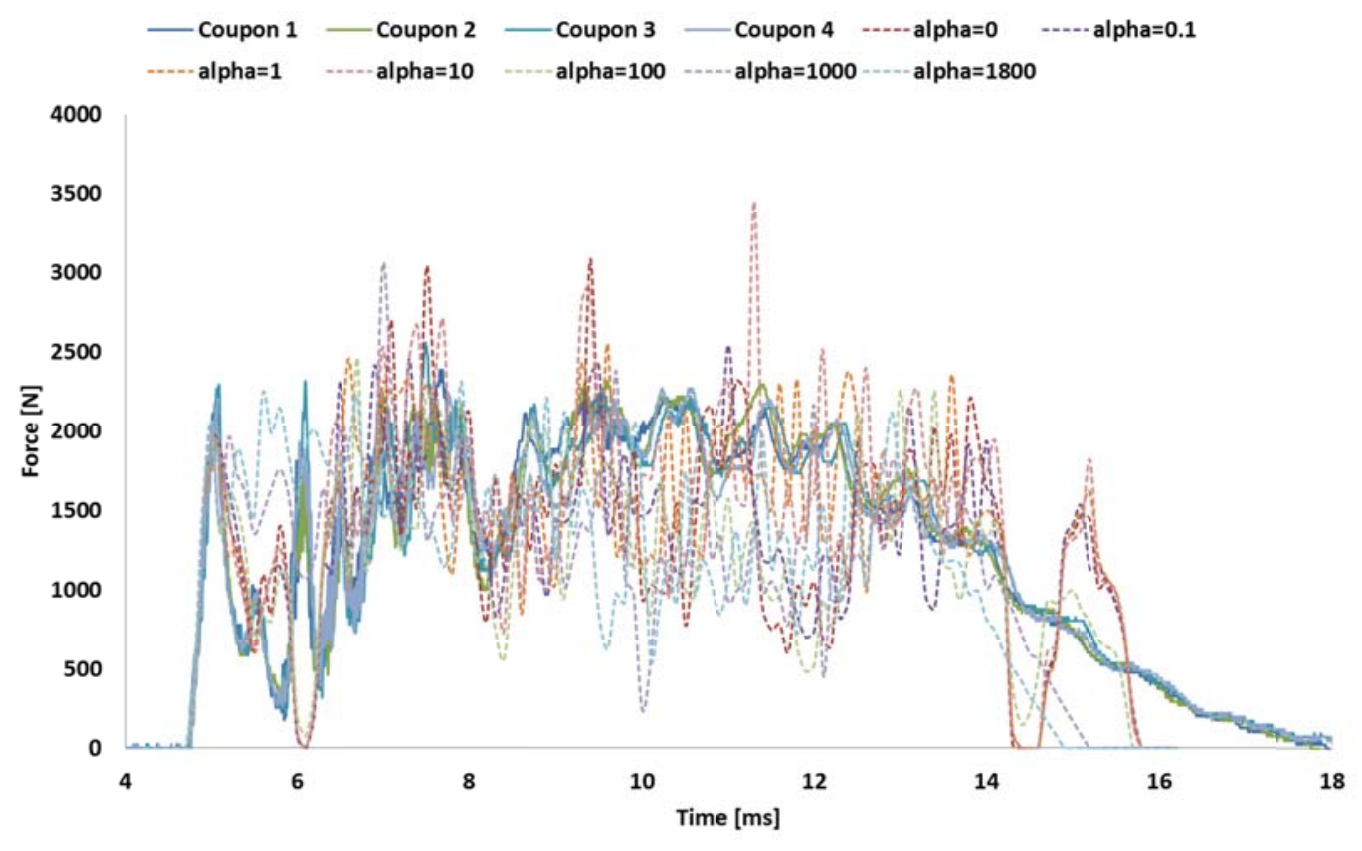

Figure 91: Damping effect for type A cylinders

To evaluate the FE model results, the variables that are compared to experimental results, are. the force peaks intensity, the time of the force peaks, frequency of oscillations and visual appearance of the FE results. For this case, the FE models with lower damping values have their force oscillations closer to the experiments as well as the time of force peak and its intensity.

As expected, higher damping parameters $(\alpha>1000)$ smooth the force in peaks and valleys, mostly from the beginning of the force history (from $4.5 \mathrm{~ms}$ to $8.5 \mathrm{~ms}$ ). On the other hand, those values better simulated the end part of the impact event (after $12.5 \mathrm{~ms}$ ). For smaller $\alpha$ values, the impact initial part was better modeled as well as the high frequency force oscillations, despite the fact that the simulation behavior for the end part diverged from the experimental results.

The simulated impact force response was considerably different even at low $\alpha$ values, for example, when $\alpha=0$, it was possible to observe two high intensity force peaks (around 7.0 $\mathrm{ms}$ and $9.0 \mathrm{~ms}$ ). Increasing $\alpha$, both the intensity and the location of the high intensity force peak changed, for example, for $\alpha=10$, the high intensity force peak took place at $15 \mathrm{~ms}$ and, it was the highest overall value.

It is also necessary to verify the influence of damping parameter on the damages variables. It has been analyzed by studying the damages parameters through the thickness in the most damaged element in the impacted area (Figure 92). 


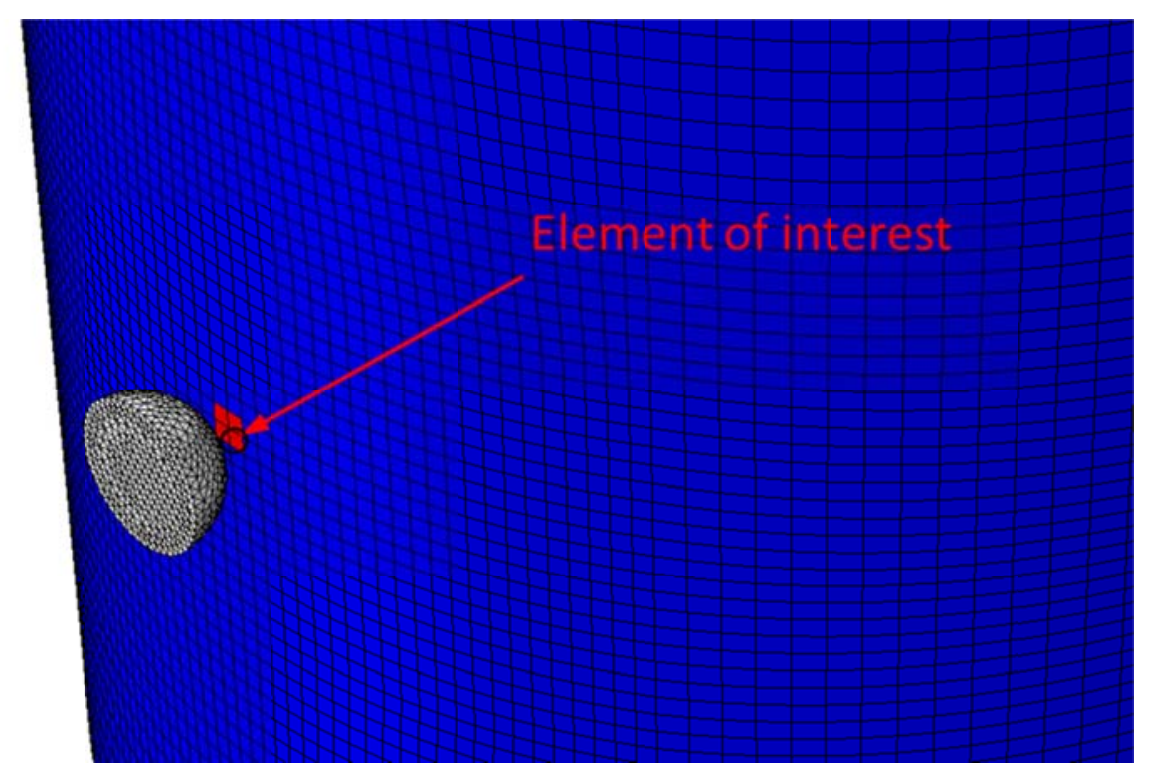

Figure 92: Mesh elements for investigation of the damping parameter influence on damage variables.

The effect of $\alpha$ on the fiber damage $\left(\mathrm{d}_{1}\right)$ is shown in Figure 93 for type A cylinder. It can be observed that even for small values of damping ( $\alpha=0.1$ ), the fiber damage differs from the no damage one.

When $\alpha=0.3$, integration points through the thickness were capable to detect $\mathrm{d}_{1}$. For $\alpha>0$ the number of integration points, which were capable to detect $\mathrm{d}_{1}$, decreased. For $0.1 \leq \alpha \leq 10, \mathrm{~d}_{1}$ was detected by two integration points, but for $\alpha=100$, only one integration point detected the fiber failure and, for $\alpha \geq 1000$, fiber damage was no longer detected by any integration point (Figure 93). The horizontal lines in this figure represent the laminate layers, thus it has been possible to observe the damage intensity in each layer.

Damping is a global model characteristic, which dissipates the impact energy on the whole model. On the other hand, damage is a local characteristic, but since the impact energy was dissipated by the whole model, less energy was available to trigger the damage process. 


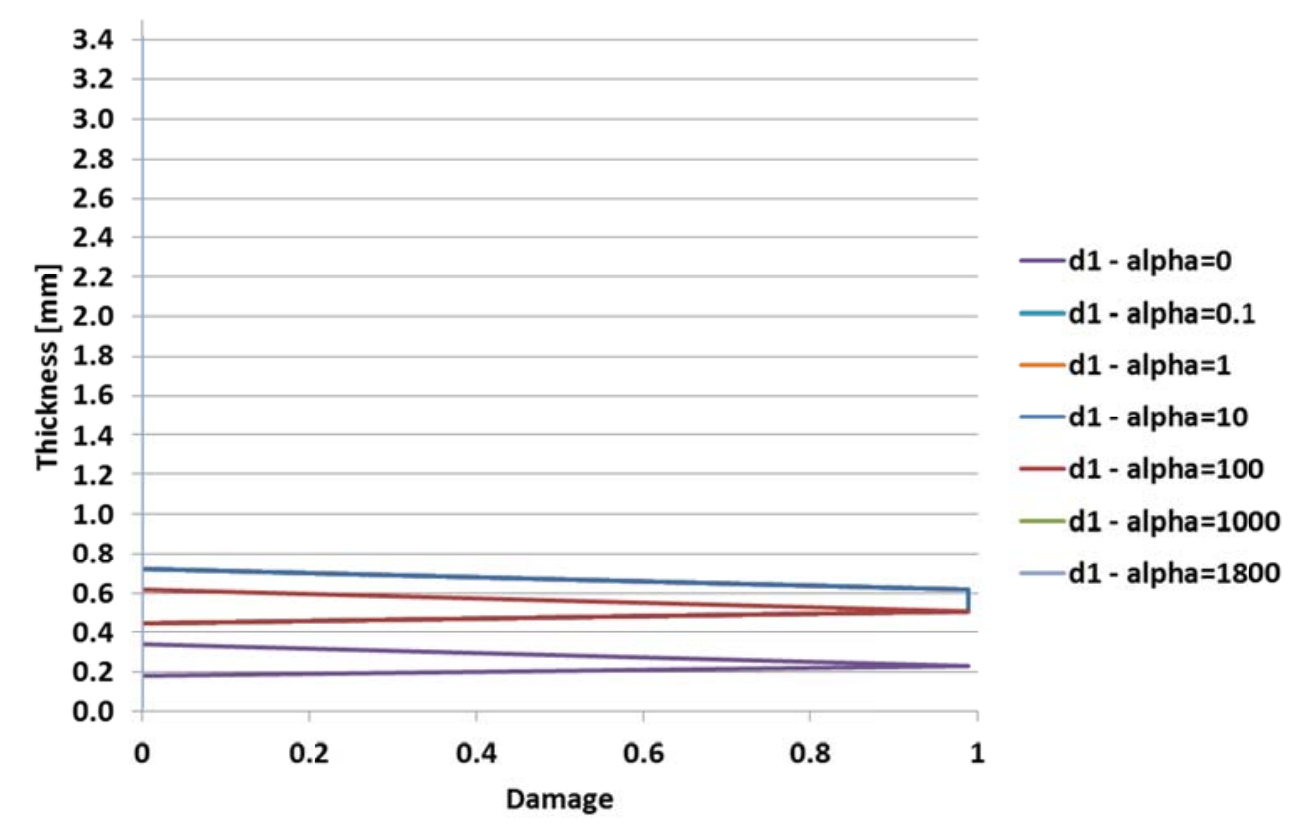

Figure 93: Type $\mathrm{A} \mathrm{d}_{1}$ damage intensity for different $\alpha$ values.

It is important to mention that for fiber compression, the effect of the damage process has been accounted by the secant modulus as established by EQ. (15).

Considering the effect of damping parameter on $d_{2}$ for type A cylinders, as for the previous case, when $\alpha=0$, the number of integration points through the thickness, which was capable to detect $d_{2}$, was higher, and the damage was relatively more intense (Figure 94). The intensity of $\mathrm{d}_{2}$ for each integration point decreased as damping increased, regardless the points where $\mathrm{d}_{2}$ had its maximum value.

It can be observed that for some internal layers, there has been no damage (from 1.6 to 2.0 $\mathrm{mm}$ ), regardless the damping parameter. Furthermore, in the layers between 2.2 up to $3.0 \mathrm{~mm}$, the damage intensity for $0.1 \leq \alpha \leq 10$ had shown a different trend, i.e. the damage intensity for $\alpha=10$ is higher than for $\alpha=1$, and this behavior was repeated until $\alpha=0.1$. Moreover, the external layers have been completely damaged in all damping parameters. This observation is the opposite of what was expected. Once the cylinder had a higher damping, there was less energy available to produce damage evolution. This difference was due to how the damping directly affects the cylinder dynamic and indirectly influences on the stress state in the shell element. It is important to remember that $\mathrm{d}_{2}$ only evolves for tension transverse stress.

As expected, the outer layers are more damaged than the internal ones. This is due to cinematic model used for thin shell elements, where the displacements are proportional to the 
middle plane distance. The derivative of the displacements regarding this cinematic model yields larger strains in the outer layers and so do higher stresses in those layers.

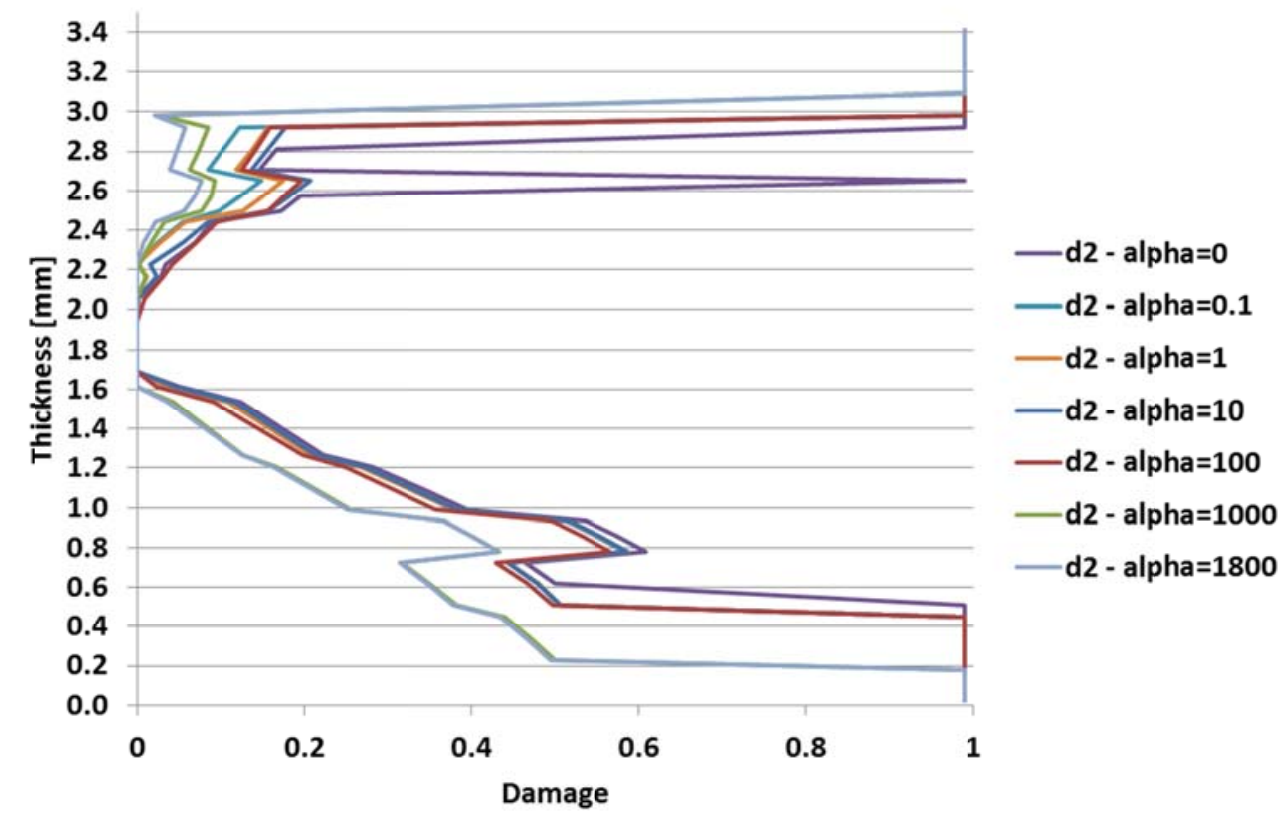

Figure 94: Damage effect on $d_{2}$ damage variable for type A

Figure 95 shows the effect of damping on the shear damage variable $\mathrm{d}_{6}$. Once again, as damping increases, the damage intensity decreases. In the cases without damping and $\mathrm{d}_{6}=1$ (fully damaged), it has been detected damage by three other integration points in addition to the cases with damping at the same point $(2.63 \mathrm{~mm})$. It is important to mention that the parameter $\mathrm{d}_{6}$ is no higher than 0.11 in the cases with damping.

The shear damage variable behaviors were very similar to $0.1 \leq \alpha \leq 100$. To $\alpha \geq 1000$, the behavior of integration points through the thickness diverged from the case with lower damping as well as for no damping. These differences are more noticeable in the layers at 0.2 to $0.6 \mathrm{~mm}$ and 2.8 to $3.0 \mathrm{~mm}$, where the high damping factor decreased the damage.

In the internal layers (from 1.8 to $2.6 \mathrm{~mm}$ ), the damping did not affect significantly the damage calculation. It is important to verify that these layers are closer to the layer, where the impactor head hits the cylinder. On the other hand, in the inner layers (from 0.2 to $1.6 \mathrm{~mm}$ ), the differences were more noticeable, mostly for high damping values. The external layers of the studied element were completely damaged in all cases. 


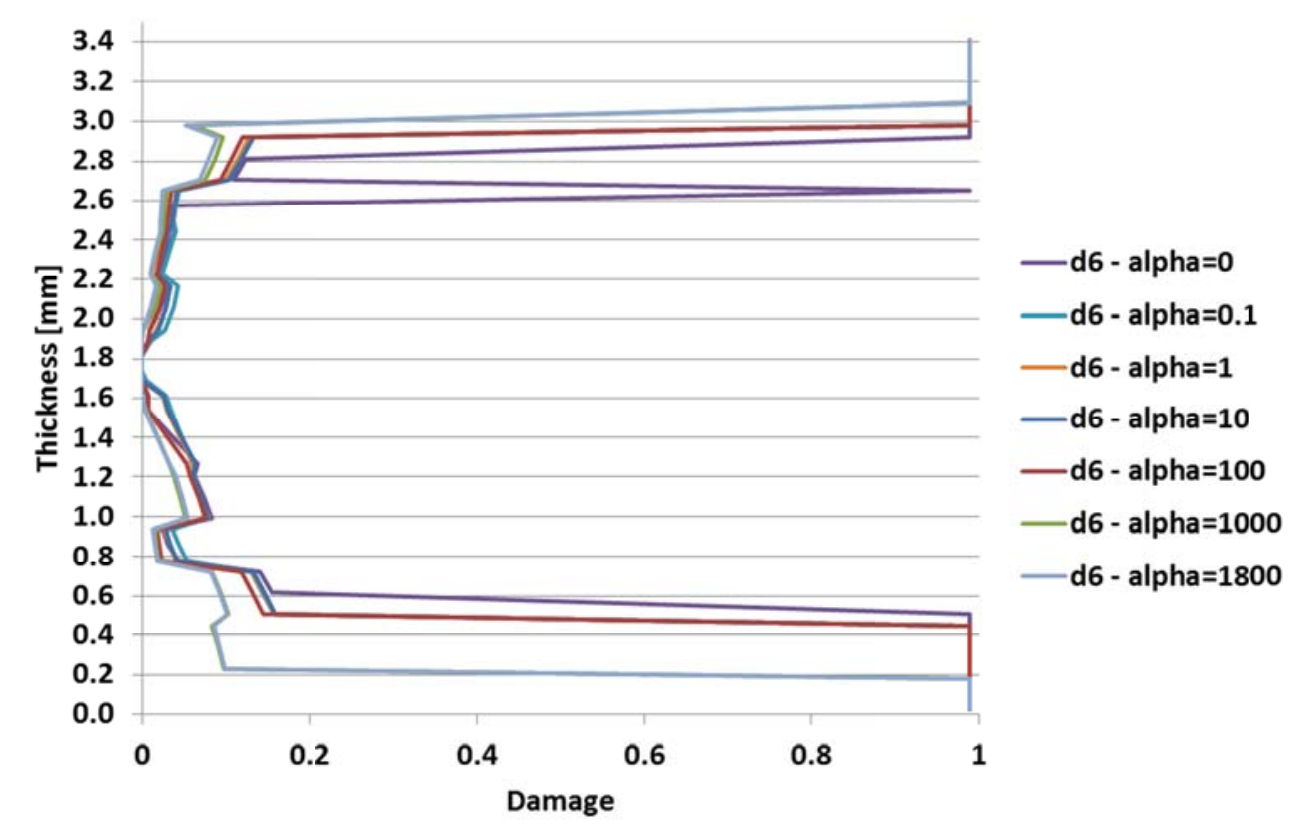

Figure 95: Damage effect on $d_{6}$ damage variable for type A

In type A cylinders, as the damage increased the damage intensity (fiber and matrix) decreased. Moreover some important failure modes, e.g. fiber failure, could be underestimated. Thus, higher damping values should not be used in the numerical simulations, despite the improvement of the predictions for the final part of the impact analyses.

On the other hand, regarding the overall simulations behavior, the best results have been obtained for $\alpha=1.0$ (Figure 96), considering a compromising between the damage calculation and the FE force $v s$. time history to improve the final part of the impact event. For this damping parameter, the first load peak was captured, but the second experimental load peak corresponded to a null simulated force $(6.5 \mathrm{~ms})$. After that, the next simulated peak corresponded to a valley for the experiments and then the simulated force decreased to lower level $(6.8 \mathrm{~ms})$. Afterwards, the simulated force increased again, making a small force platform (from $6.9 \mathrm{~ms}$ to $7 \mathrm{~ms}$ ). This force platform corresponded to the load cell force platform obtained from the experiments.

The next experimental force valley $(8.5 \mathrm{~ms})$ corresponded to a simulated force valley. At this simulation time, the FE model detected the fiber failure onset (Figure 96). After this valley, the force increased again and, the experimental force oscillations were lower than the simulated forces. From $9.0 \mathrm{~ms} \mathrm{~s}$ up to $12.5 \mathrm{~ms}$, the experimental force oscillated around 2000 
$\mathrm{N}$. On the other hand, the simulated forces oscillated around $1800 \mathrm{~N}$, but at a higher amplitude.

From $12.5 \mathrm{~ms}$, the simulation results diverged from the experimental results. The experiments had a smoother final part and the simulation final part has been suddenly reduced from $1500 \mathrm{~N}$ to $0 \mathrm{~N}$ in a very short time. Then, the simulated force remained null for $0.2 \mathrm{~ms}$ and increased again up to $1800 \mathrm{~N}$ rather fast. Subsequently the force decreased again up to 0 $\mathrm{N}$, finishing the simulation process at $16.0 \mathrm{~ms}$. However, the experimental force history ended at $18.3 \mathrm{~ms}$.

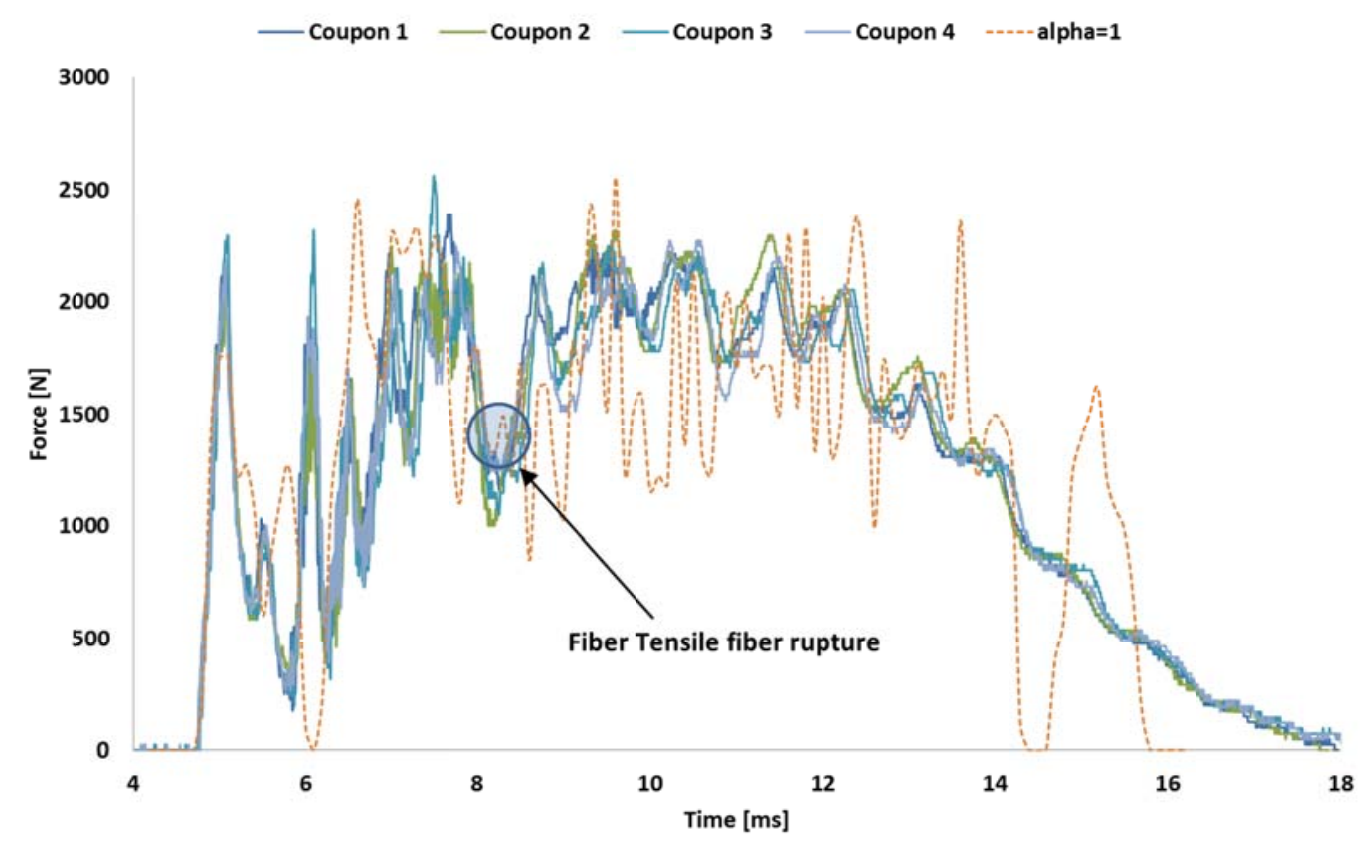

Figure 96: Type A best alpha parameter

\subsection{Type B damping effect}

The cylinder lay-ups had been chosen to evaluate the model for different composite configurations (orthotropic degree). Thus the influence of damping must also be checked for this lay-up, once the damage calculation is a function of the ply orientation. Type B cylinders simulation results are shown in Figure 97.

As for cylinders type A, the FE model used both the intermediate mesh refinement (Figure 87(b)) and the contact algorithm for the cylinder and impactor head interaction was the general contact with hard contact normal behavior as well as Penalty Method for tangential behavior. The damping parameters have been the same as those used in the last case. 
In order to study the damping effect on damage variables, the most critical element in the FE model has been analyzed and the damage intensity at each integration point through the thickness has been shown and discussed in this section.

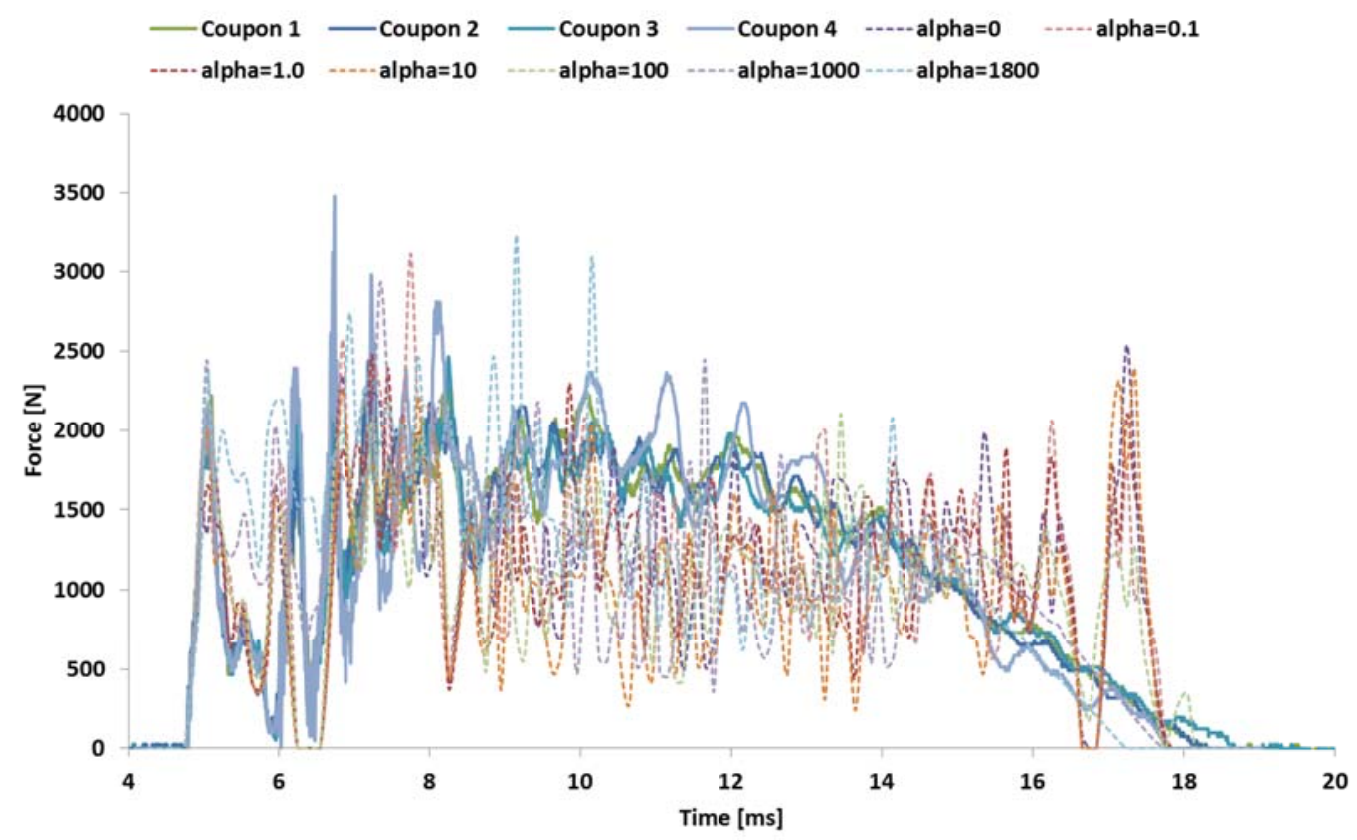

Figure 97: Type B damping effect

To evaluate the FE model results, the variables that are compared to experimental results, are. the force peaks intensity, the time of the force peaks, frequency of oscillations and visual appearance of the FE results. Once again, the FE models with lower damping values have their force oscillations closer to the experiments as well as the time of force peak and its intensity.

Again, it is possible to observe that high damping values smoothed the simulated force oscillations (from $4.7 \mathrm{~ms}$ to $6.0 \mathrm{~ms}$ ) and, increase of damping coefficient shifted the simulated maximum force peaks, for example the maximum force of $3400 \mathrm{~N}(9.0 \mathrm{~ms})$ is $3.0 \mathrm{~ms}$ delayed from the experiments. At high damping, the end part of impact (from $14.0 \mathrm{~ms}$ ) is better simulated, considering the lowest damping values.

Figure 97 helps to understand the damping effect on computational simulations, but it is also necessary to verify how the damping affects the damage calculation by analyzing the damage variables through the thickness.

Figure 98 shows how damping affects the fiber damage variable $\left(d_{1}\right)$. The increasing damping decreased the number of integration points, where $d_{1}$ had been detected and, for $\alpha \geq 1000, \mathrm{~d}_{1}$ has not been detected anymore. As mentioned before, those high damping 
values did not have physical meaning and they were only used to improve the end part of the impact response in the numerical simulations.

For type A cylinders, considering damping parameters $0 \leq \alpha \leq 10$, there have been no differences in $\mathrm{d}_{1}$ and, for $\alpha=100$, only one integration point could detect fiber damage.

Regarding the material model for fiber compression, the damage is accounted by decreasing its elastic modulus by using the secant modulus as established by EQ. (15). Thus $\mathrm{d}_{1}$ has only been detected in layers under tensile loads in fiber direction.

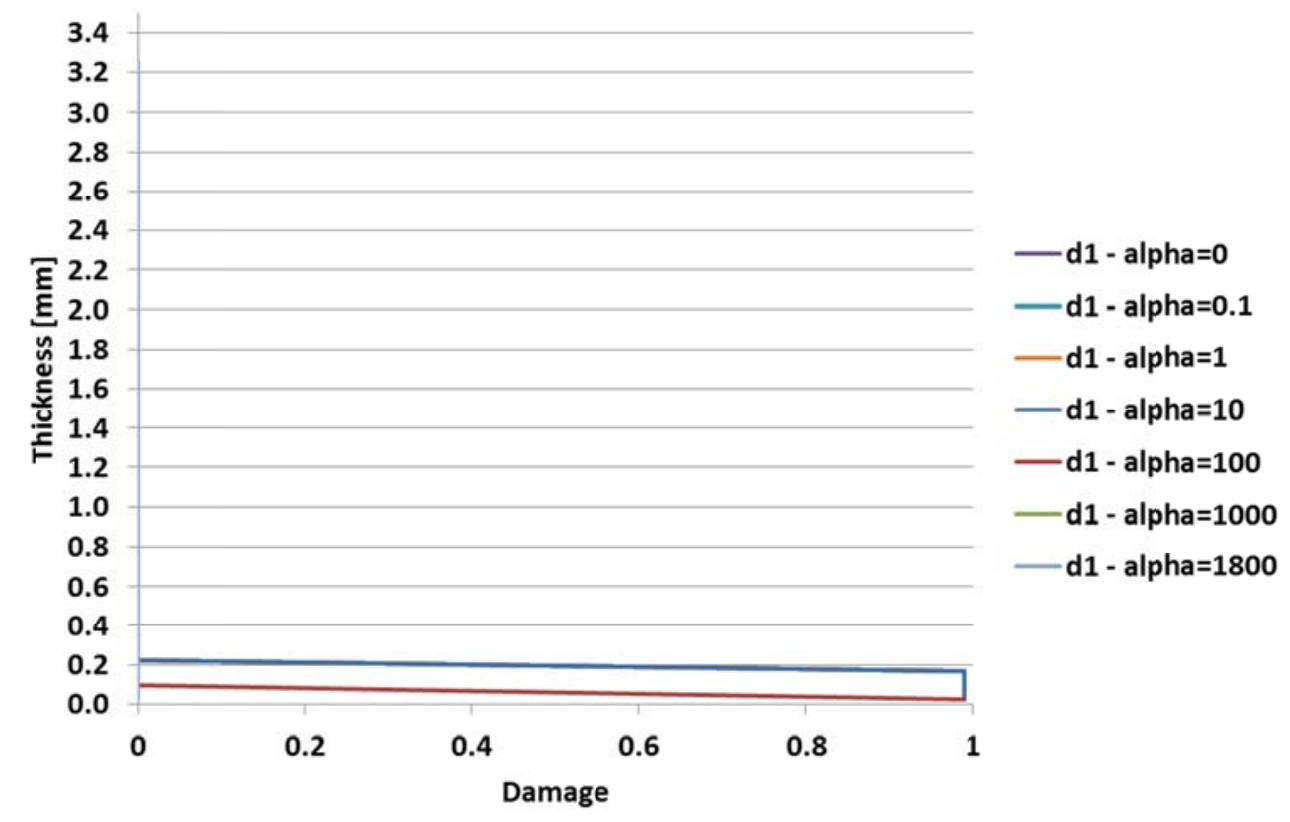

Figure 98: Damage effect on $d_{1}$ damage variable for type $B$

The damage variable $d_{2}$ through the thickness is shown in Figure 99. There have been no considerable differences in $\mathrm{d}_{2}$ in the case without damping and when $\alpha=0.1$. There were small differences not only in $\alpha=1.0$ but also in $\alpha=10.0$. On the other hand, in $\alpha>100$, the differences in damage calculation have been more pronounced and from $1.6 \mathrm{~mm}$ (Figure 99) on, this divergence has been higher. In $\alpha \geq 1000$, the model has not shown any damage.

It is possible to observe the decreasing of $\mathrm{d}_{2}$ from the external layers to the internal layers. Independent of the value of $\alpha$, there has been no damage calculated in the integration points through the thickness from $1.6 \mathrm{~mm}$ to $2.0 \mathrm{~mm}$ (Figure 99).

Furthermore, despite the fact that the external layers have been more damaged, no other one has been completely damaged for the element used in this analysis. In the inner layer $(0.0$ $\mathrm{mm}), \mathrm{d}_{2}=0.48$ and, in the outer layer, $\mathrm{d}_{2}=0.18$ for the case without damping. 


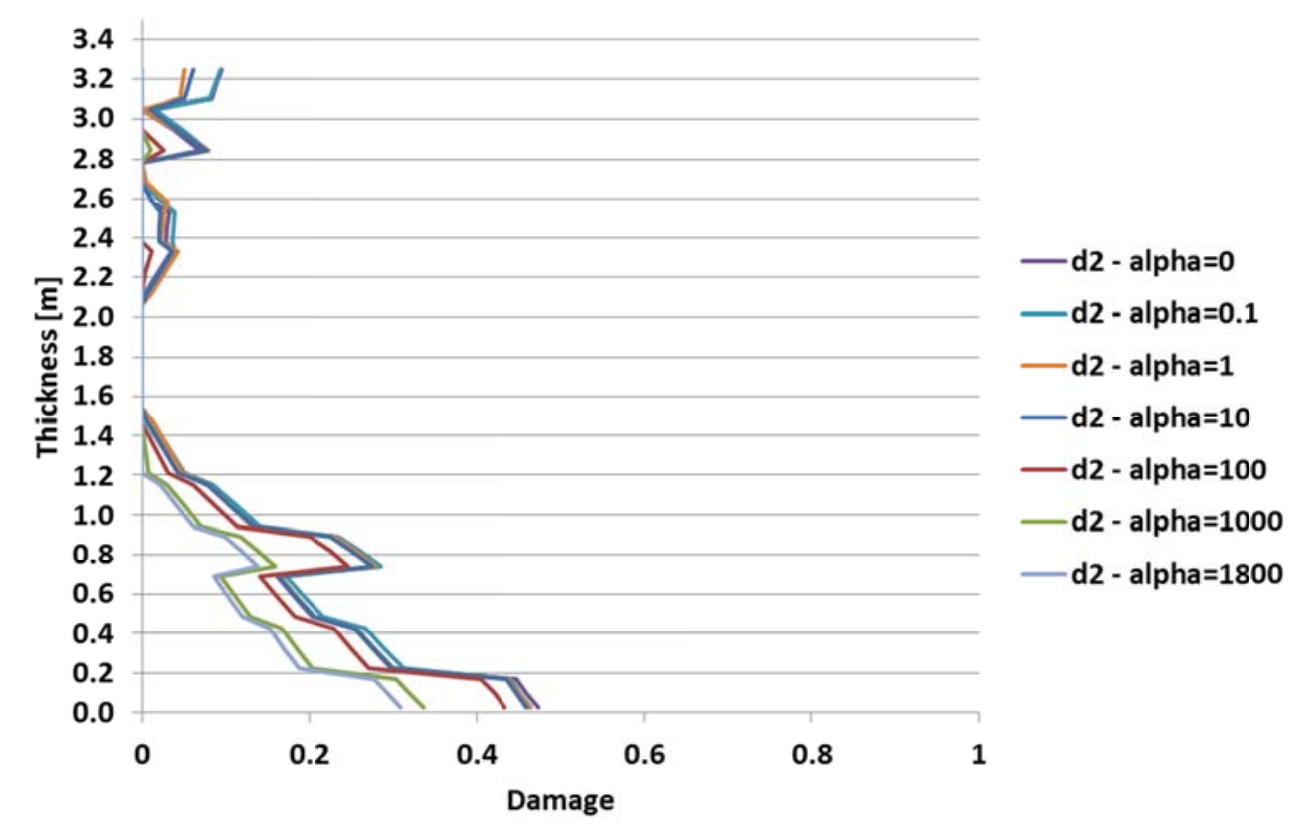

Figure 99: Damage effect on $d_{2}$ damage variable for type $B$

Based on Figure 100, it can be observed a damping effect in the shear damage variable. In fact, $\mathrm{d}_{6}$ behaves in a different way from the previous damage variables in cylinders type $\mathrm{A}$ and B.

Firstly, the most damaged layers have been internal layers, not the external layers, as found in the previous cases, i.e. layers at 0.4 to $0.6 \mathrm{~mm}$ and 2.6 to $2.8 \mathrm{~mm}$ in all damping values. Furthermore, the damage intensity is relatively lower and its maximum value of 0.16 at $0.43 \mathrm{~mm}$ is closer to the cylinder inner surface.

Despite those differences, $d_{6}$ had the same trend shown by $d_{2}$, i.e. the damage decreased as the damping increased, as well as the damage decreased towards the laminate middle plane. Moreover, for cylinder type B, the damping did not show a considerable influence on the damage calculation. 


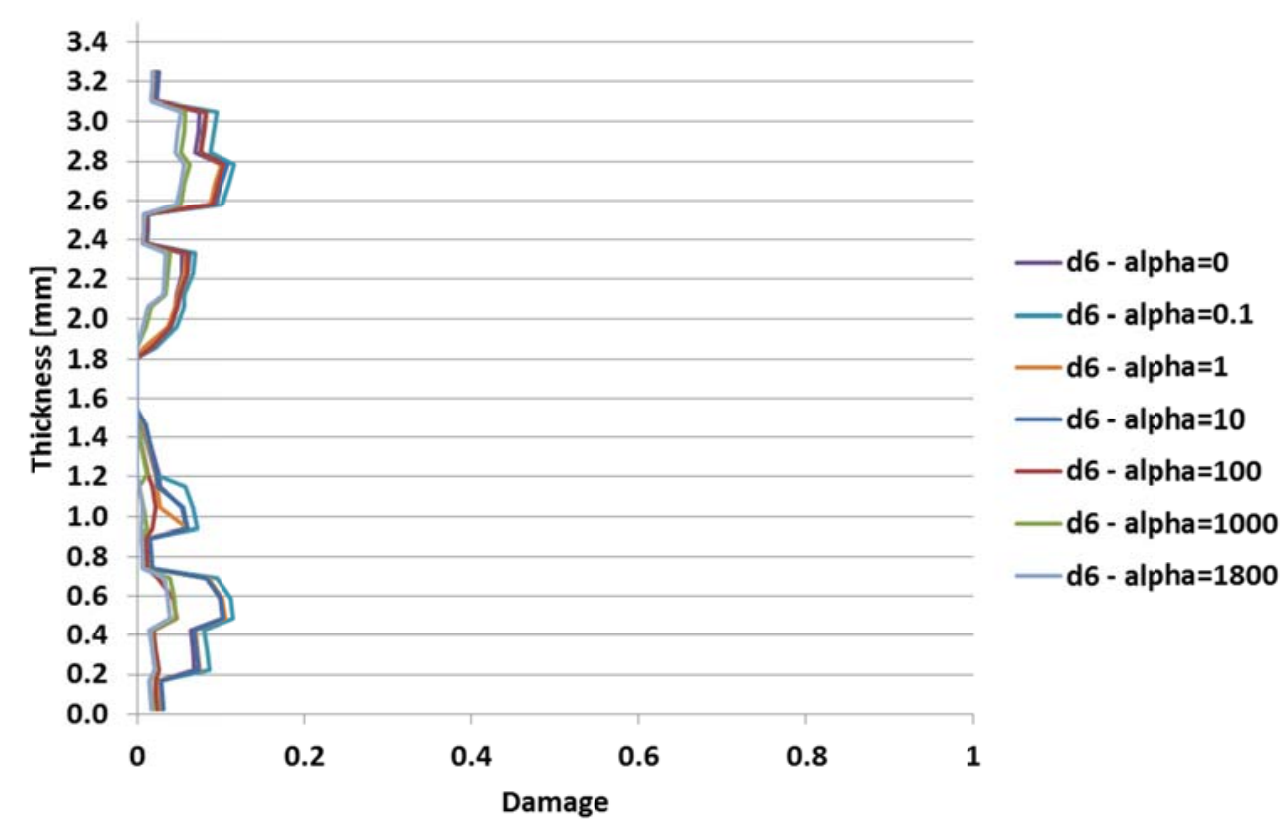

Figure 100: Damage effect on $\mathrm{d}_{6}$ damage variable for type $\mathrm{B}$

Regarding all the comments about the damping effect on damage calculation and, observing the overall computational simulation response, the best results (compromising between damage calculations and FE simulations results for force vs. time) have been obtained in $\alpha=1$ (Figure 101).

The FE model simulated the first force peak, but the force intensity was lower (1700 N for FE model and $2200 \mathrm{~N}$ for the experiments). Then the force decreased to $500 \mathrm{~N}$ and, increased again up to $1750 \mathrm{~N}$ at $6.0 \mathrm{~ms}$. This point corresponds to a null force point for the experiments. After that, the simulated force dropped to $0 \mathrm{~N}$, but the experimental force reached another peak value $(2400 \mathrm{~N})$; as a consequence the experimental force dropped to almost null value and started to increase as for numerical simulations $(6.5 \mathrm{~ms})$.

This next force peak has been the highest value for the experiments $(3500 \mathrm{~N})$ and for the simulations $(2000 \mathrm{~N})$. The difference has been rather large between the simulation and the experiments to predict the force peak intensity. From this point $(6.7 \mathrm{~ms})$ up to $12.7 \mathrm{~ms}$, the differences between the experimental and simulation results have not been higher than those shown by the maximum force peak intensity. Furthermore, the fiber fails onset at $11.2 \mathrm{~ms}$, which was latter when compared to the fiber failure onset for cylinders type A.

The ending of the impact for the experiments (from $12.7 \mathrm{~ms}$ ) is much smoother than for the simulations, where the force dropped to $0 \mathrm{~N}$ and increased again to high load levels (1500 
$\mathrm{N})$ for three times until completing the impact event. In this case, the impact simulation time was almost the same as the experimental impact time.

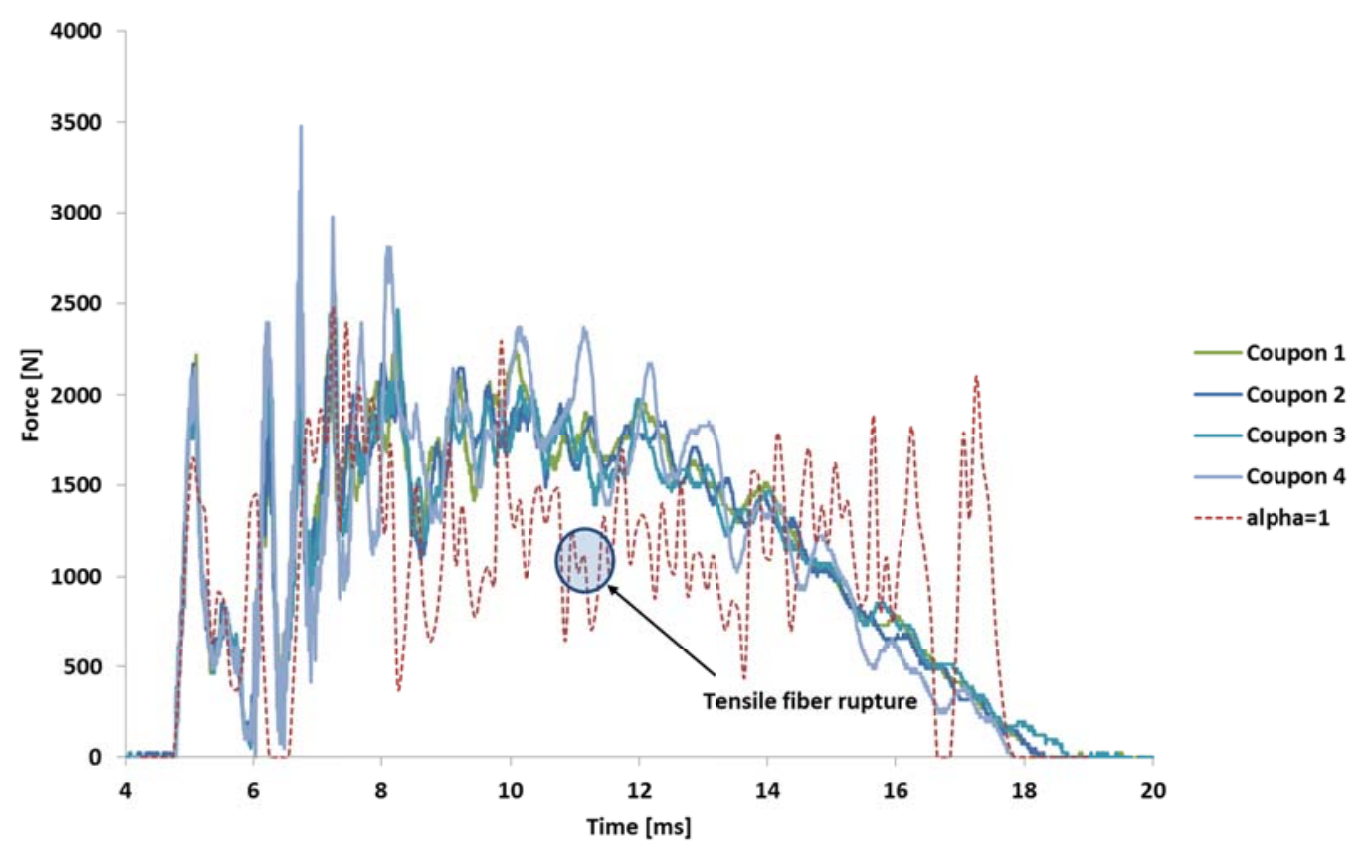

Figure 101: Type B: the best alpha parameter.

\subsection{Type C damping effect}

The damping effects on damage calculation for type $\mathrm{C}$ cylinders are discussed in this section and the results are presented in Figure 102. 


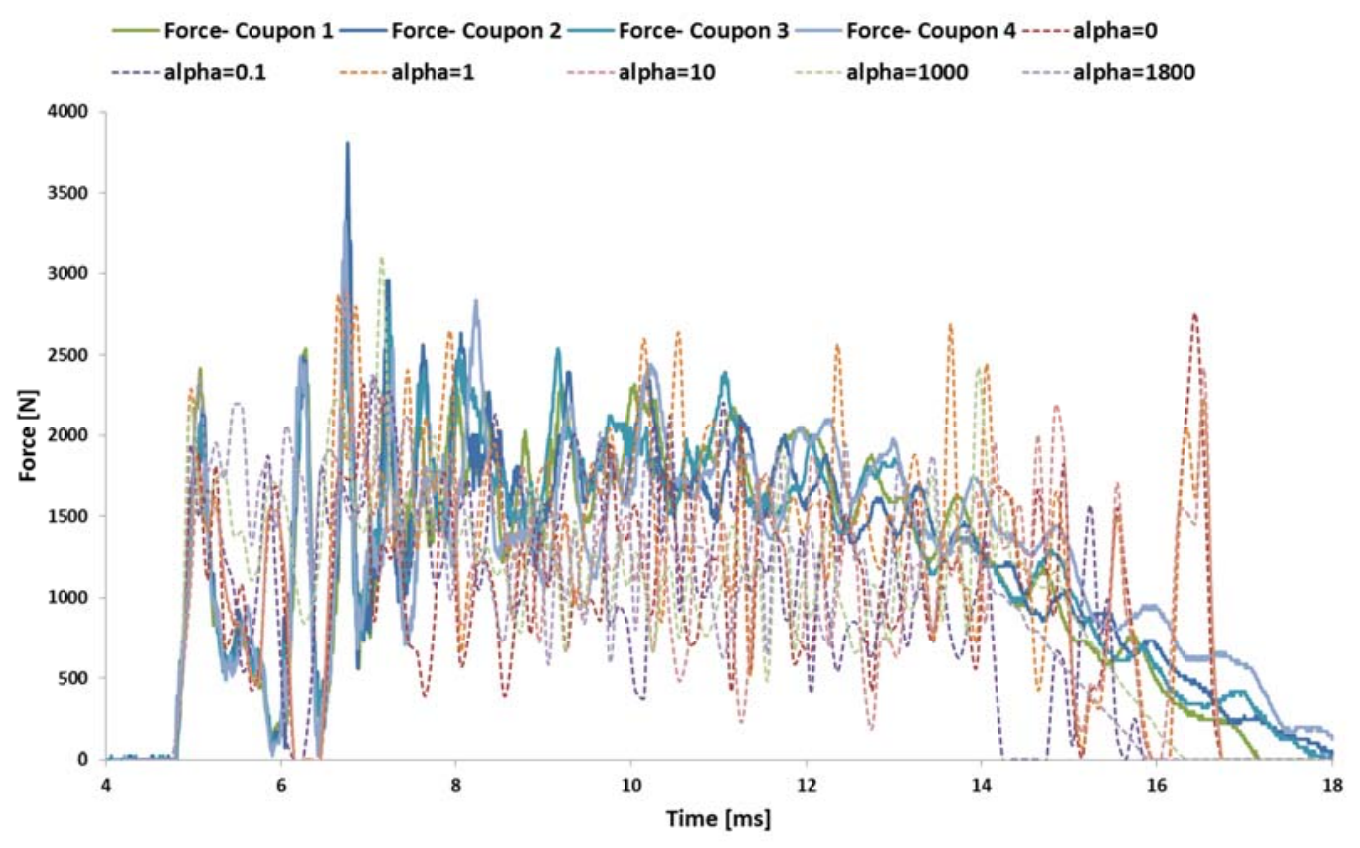

Figure 102: Type $\mathrm{C}$ damping effect

To evaluate the FE model results, the variables that are compared to experimental results, are. the force peaks intensity, the time of the force peaks, frequency of oscillations and visual appearance of the FE results. As for the previous both cases, the FE models with lower damping values have their force oscillations closer to the experiments as well as the time of force peak and its intensity.

Type $\mathrm{C}$ cylinders behave similarly to type $\mathrm{A}$ and $\mathrm{B}$ cylinders, i.e. as the damping increased, the oscillations frequency decreased as well as the end part was smoother. Once again, the effect of damping on damage calculation must be assessed. Thus, the damping effect on the damage variables through the thickness for the most critical element (Figure 92) has also been analyzed.

For type $\mathrm{C}$ fiber damage, the damping parameter has not changed $\mathrm{d}_{1}$ through the thickness in $\alpha \leq 1$ and, $\mathrm{d}_{1}$ has been detected in three integration points. In the next damping value, $\mathrm{d}_{1}$ has been detected in only two integration points and, in $\alpha=100$, in only one integration point. Considering greater values of $\alpha$, the fiber damage has not been detected (Figure 103). 


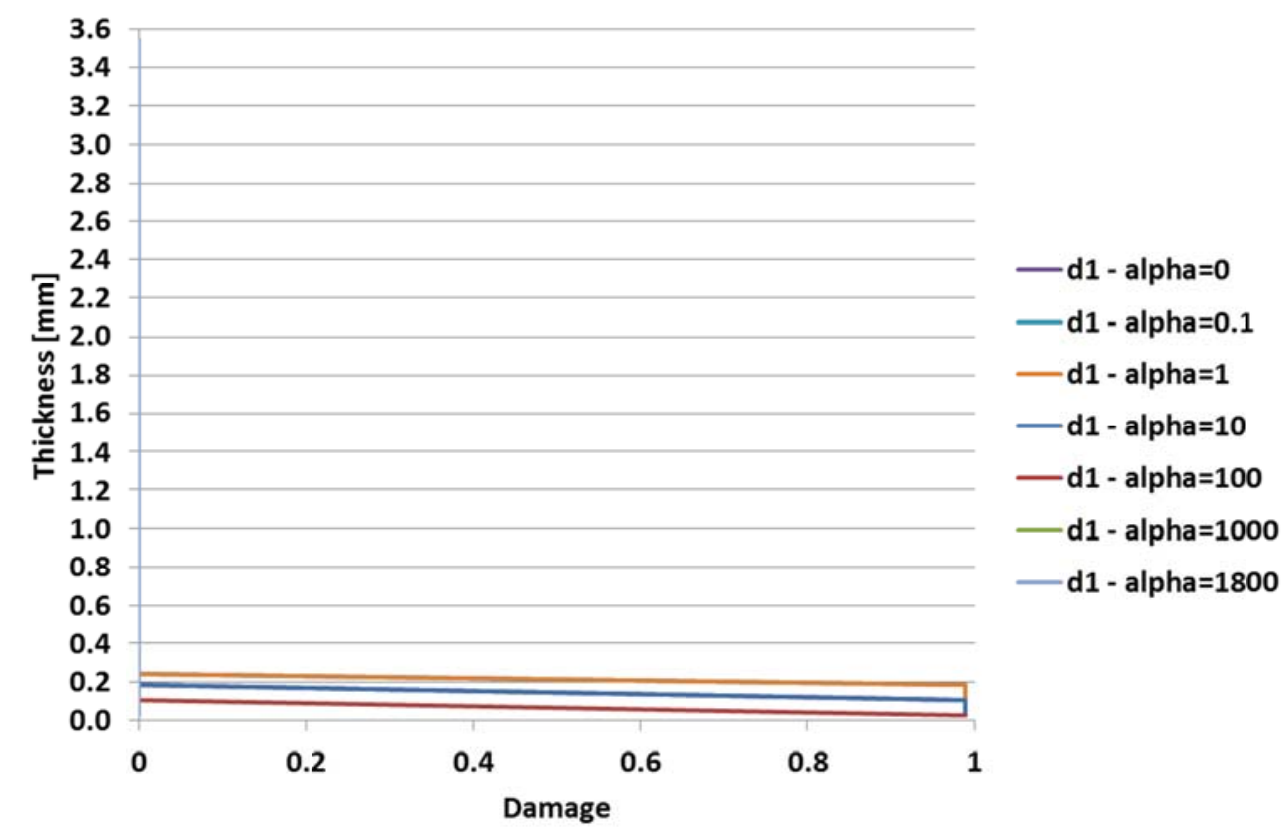

Figure 103: Damage effect on $\mathrm{d}_{1}$ damage variable for type $\mathrm{C}$

For $\mathrm{d}_{2}$, the damping affected the damage calculation through the thickness in a different way (Figure 104), i.e. in the inner layers (between 0 up to $1.6 \mathrm{~mm}$ ), the damping did not modify the damage calculation strongly and, for $\alpha \leq 10$, the differences were negligible. From 1.6 to $2.6 \mathrm{~mm}$, there was no $\mathrm{d}_{2}$, despite damping factor.

From $2.6 \mathrm{~mm}$ on, $\mathrm{d}_{2}$ has been significantly affected by damping. It can be observed that for $\alpha=100$, only two integration points detected $\mathrm{d}_{2}$ and, for higher damping, there was no $\mathrm{d}_{2}$. On the other hand, for lower damping factors, the damage calculation was different for each $\alpha$ and, for $\alpha=10, \mathrm{~d}_{2}$ reached its maximum value (from 2.6 to $3.6 \mathrm{~mm}$ ) in the external layer (3.6 $\mathrm{mm})$.

It is important to verify that none of the layers was completely damaged and the maximum $\mathrm{d}_{2}$ was equal to 0.48 at $0.0 \mathrm{~mm}$. 


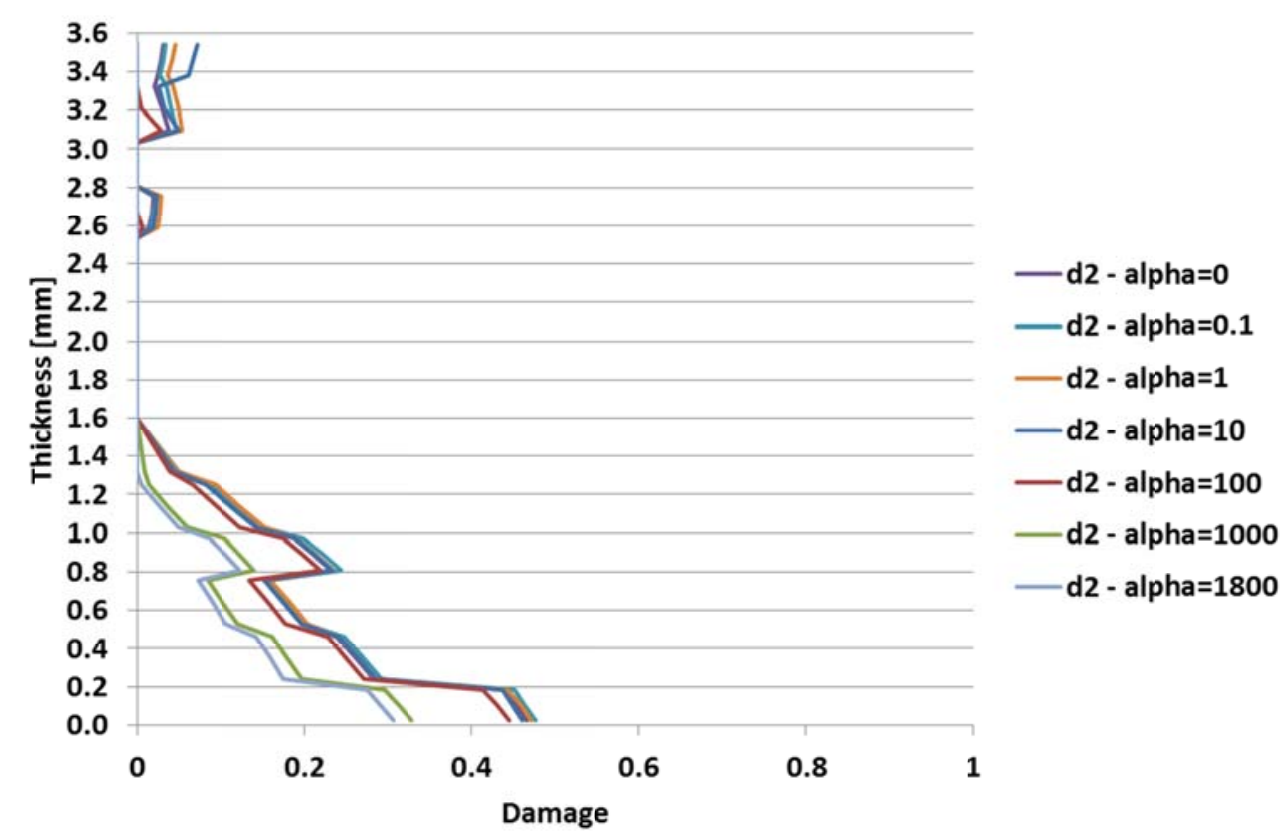

Figure 104: Damage effect on $\mathrm{d}_{2}$ damage variable for type $\mathrm{C}$

Figure 105 shows the damping effects on the shear damage calculation. It is important to observe that the most damaged layers have been between 0.4-0.6 $\mathrm{mm}$ and 2.8-3.0 mm. Furthermore, the central part (from 1.6 to $1.9 \mathrm{~mm}$ ) has not been damaged.

The highest differences caused by damping on the shear damage have been detected between 0.2 to $0.8 \mathrm{~mm}$. For these layers, $\mathrm{d}_{6}$ has been underestimated for $\alpha>1$. Between 1.9 to $3.25 \mathrm{~mm}$, the damage has been considerably underestimated for $\alpha \geq 1000$. It is important to verify that the shear damage intensity has been relatively small, because $d_{6}=0.16$ at $0.5 \mathrm{~mm}$ for $\alpha \leq 1$. 


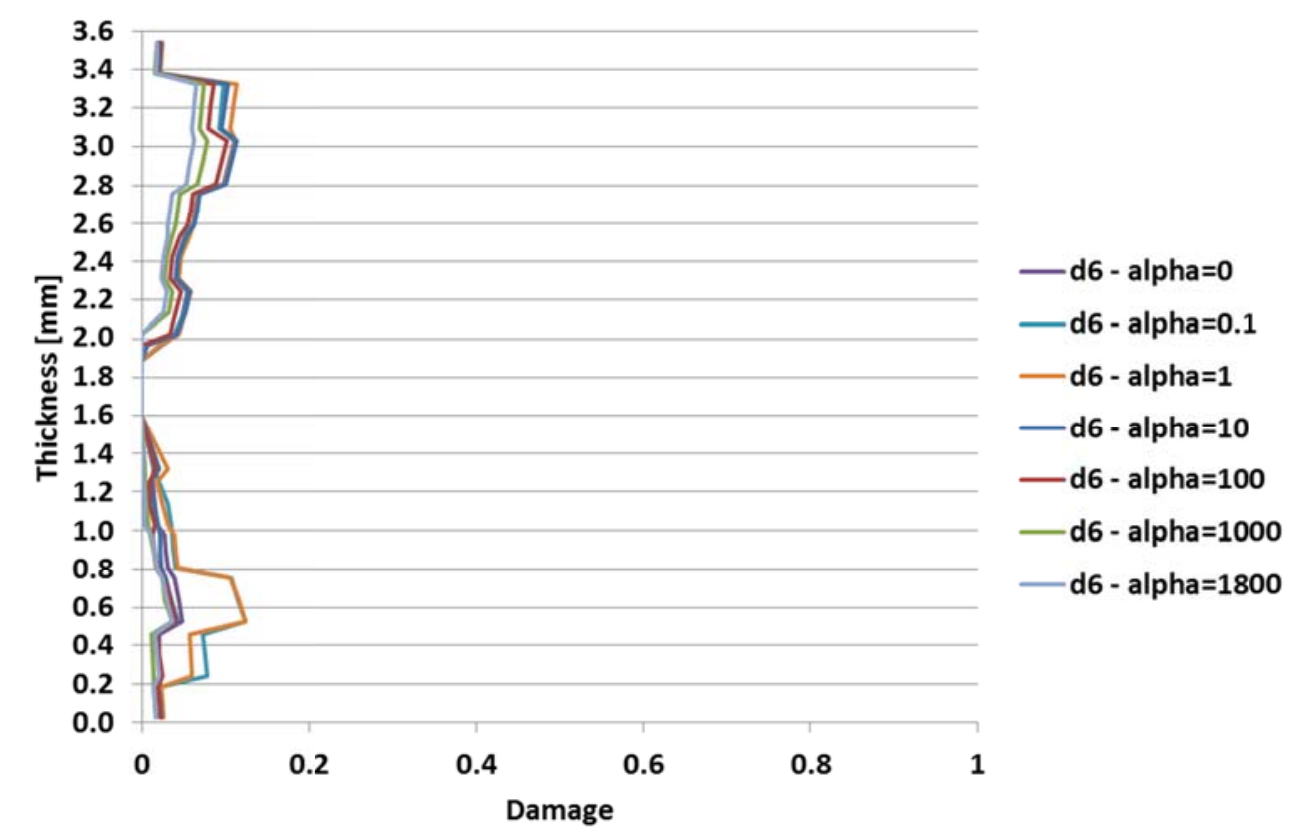

Figure 105: Damage effect on $\mathrm{d}_{6}$ damage variable for type $\mathrm{C}$

Regarding the aforementioned remarks for damage calculation and the overall behavior for type $\mathrm{C}$ impact simulations, again, the best results have been for $\alpha=1$ (Figure 106), since the damage calculation has not been significantly affected and the FE force vs. time results have been good, regarding the criteria (intensity of force peak; time of the force peak occurrence and frequency of oscillations).

In this case, the first force peak intensity was well simulated by the model, then the forces decreased until certain value and increased again, forming an intermediate peak and later, they dropped again. The next force peak was different for both the FE model (at $5.5 \mathrm{~ms}$ ) and the experiments (at $6.5 \mathrm{~ms}$ ). After this second force peak, the force dropped to $0 \mathrm{~N}$ (FE model and experiments) and the force suddenly increased to its highest value (2800 N for FE model and $3800 \mathrm{~N}$ for experiments) for both simulation and experiments.

From this peak, for simulation, the force dropped until $2000 \mathrm{~N}$ and, then, increased again up to $2750 \mathrm{~N}$, forming two peaks, while the experiment had only one peak. After this period of high amplitude oscillations (from $4.7 \mathrm{~ms}$ to $7.0 \mathrm{~ms}$ ), the force was still oscillating, but the amplitude for the experiments was smaller than that in the FE model. At this simulation part, the FE model detected the fiber damage onset. It is important to verify that the fiber failure onset did not correspond to a high force peak.

As for the other simulations, the results for the final part (from $0.0130 \mathrm{~s}$ ) diverged significantly from the experiments. 


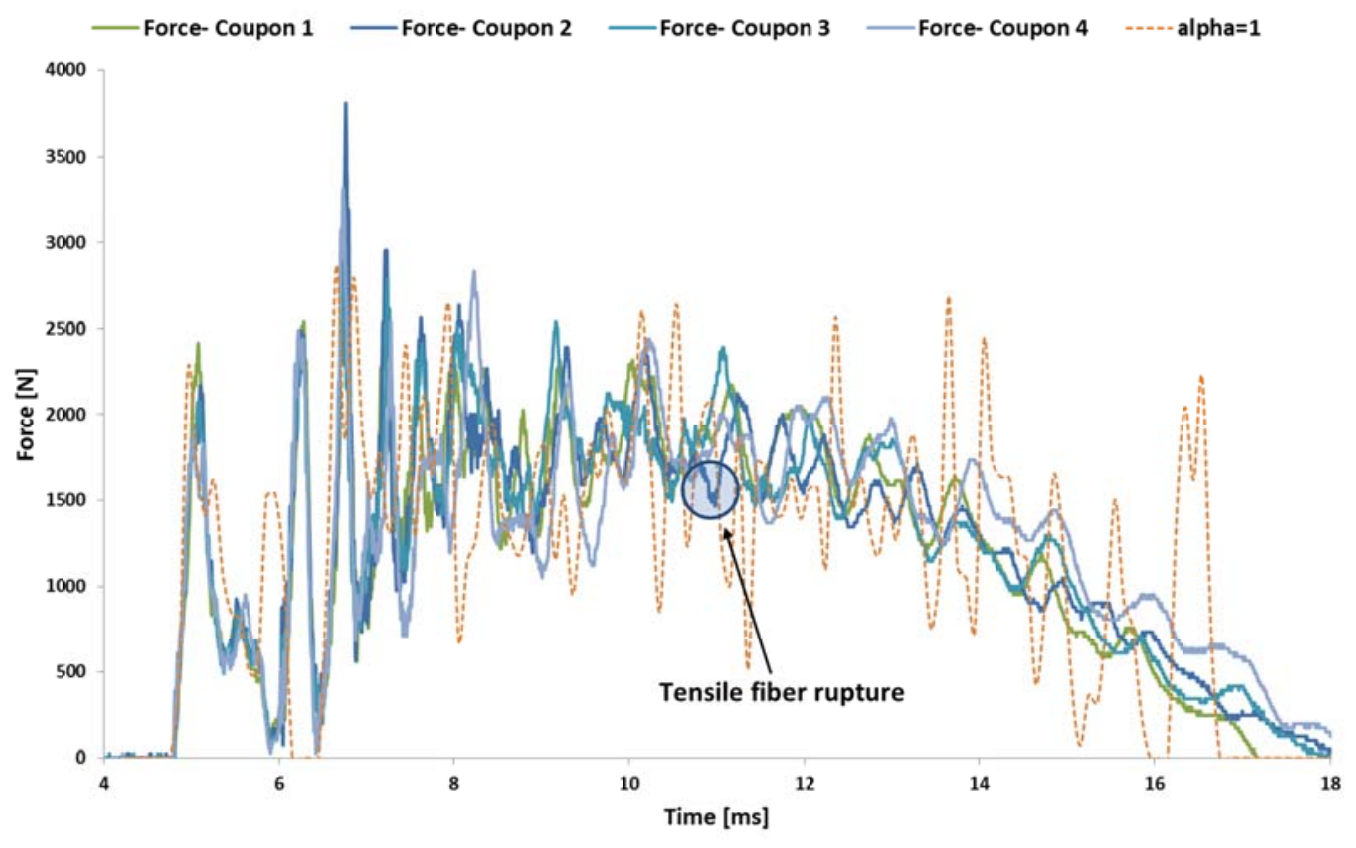

Figure 106: Type C best alpha parameter

Finally, it is confirmed that the damage calculations are a function of the ply orientation; thus they depend not only on the ply stress state, but also on the damping effects.

\subsubsection{RESULTS AND DISCUSSIONS}

As shown in the previous sections, several numerical simulations have been performed for each cylinder lay-up in order to identify "the best" FE model. The results for "the best" configuration are shown in the following sections, considering the values of parameters, which have been investigated.

\subsubsection{Damage vs. No damage.}

In order to check how the damage model affects the impact force response, the following analysis shows a comparison between the FE model, regarding the damage model and the same FE model without damage (i.e. elastic) calculation. The mesh presented in Figure 87(b), as well as general contact with hard contact for normal behavior and no damping effects have been used in those simulations. 
For cylinders type A (Figure 107), the beginning of the impact event (from $4.5 \mathrm{~ms}$ to 6.0 $\mathrm{ms}$ ) has shown a very similar behavior for both the cases (damage and no damage). From 6.0 $\mathrm{ms}$, the damage increased due to the proposed material model, and it was possible to observe that the force intensity was considerably higher for the elastic modell than for the damage model proposal ( $1600 \mathrm{~N}$ for damage and $2800 \mathrm{~N}$ for elastic model). From $6.5 \mathrm{~ms}$ s to $14.5 \mathrm{~ms}$, the behavior between the damage model and the elastic model was different. At the end of the numerical simulation (from $14.5 \mathrm{~ms}$ to $16.0 \mathrm{~ms}$ ), both the models behaved similarly again.

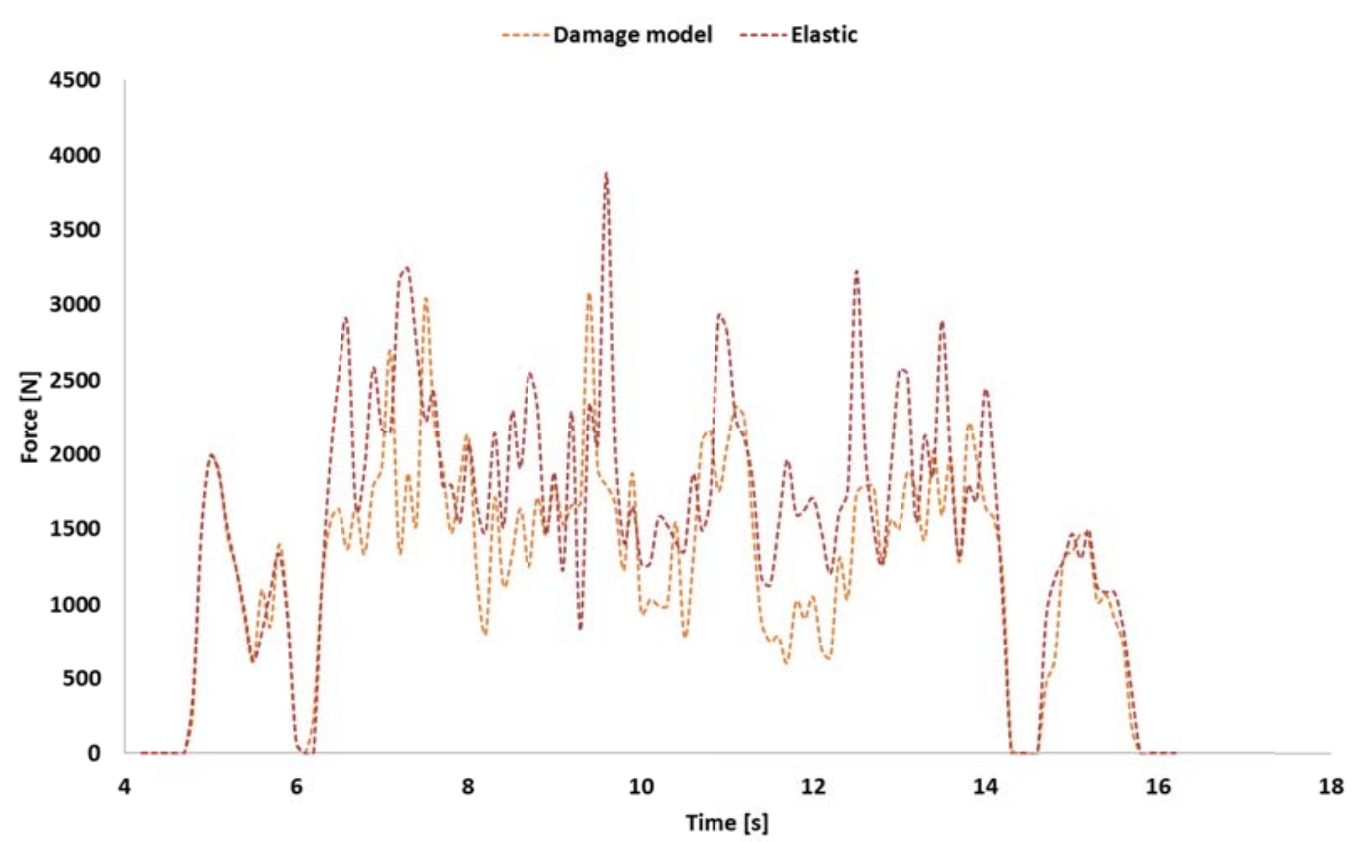

Figure 107: Type A cylinders: Elastic Model vs. Damage Model results (without damping effects).

In type B, the effect of damage in the force intensity of peaks and valleys is more pronounced than in type A (Figure 108). It can be observed that the results diverged from the beginning, when the first force peak was different. After this first peak, both models behaved similarly until $6.5 \mathrm{~ms}$. From this point, the elastic model force peak $(3000 \mathrm{~N})$ was considerably higher than the damage model force peak $(2400 \mathrm{~N})$ and, both models behaved rather different until the end of the impact event.

Regarding the elastic model, the force level for the time interval from $7.0 \mathrm{~ms}$ to $16.0 \mathrm{~ms}$ is relatively higher than for the damage model, considering this time interval. 


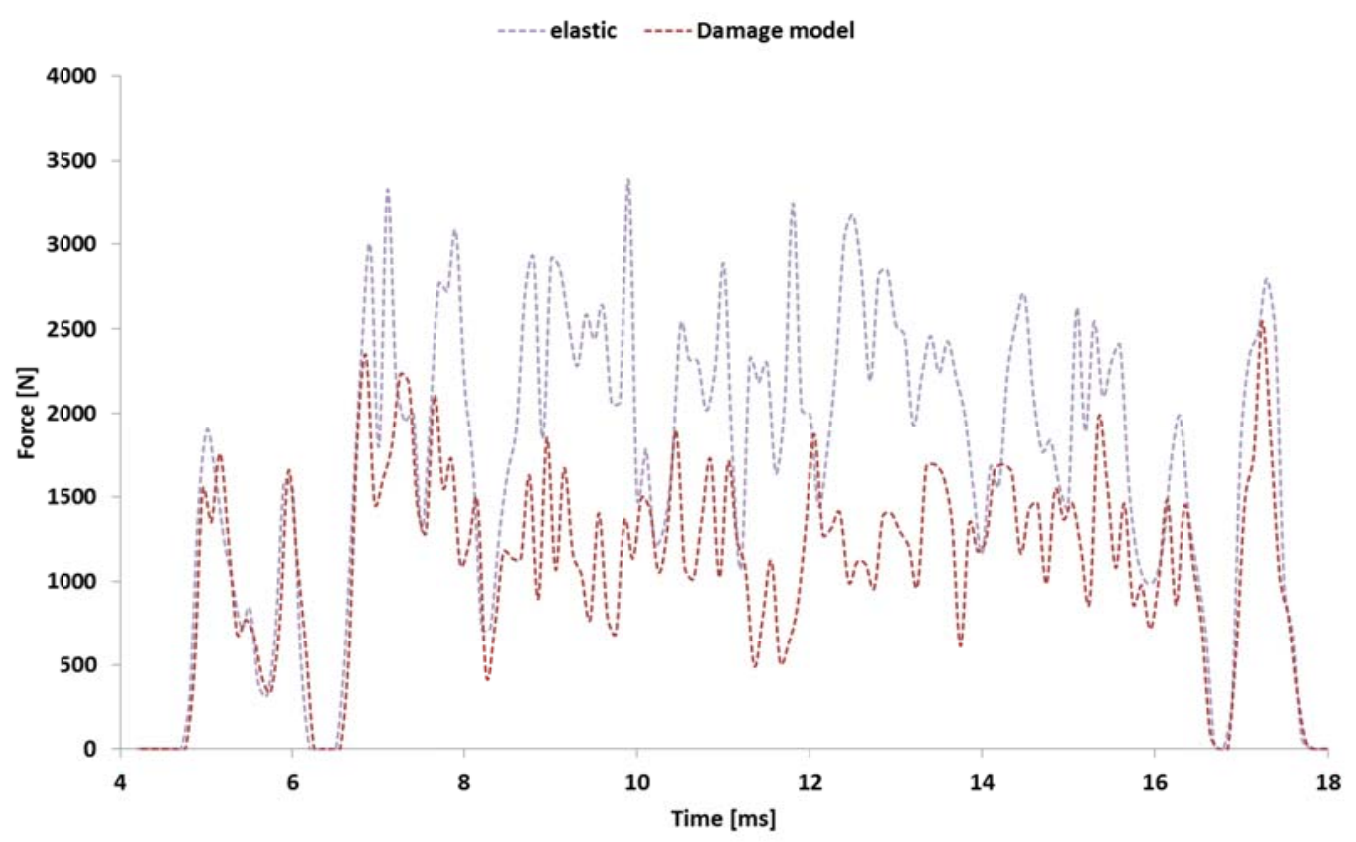

Figure 108: Type B cylinders: Elastic Model vs. Damage Model results (without damping effects).

The analysis of the damage effect on the force response for cylinders type $\mathrm{C}$ is shown in Figure 109. From $4.7 \mathrm{~ms}$ to $7.0 \mathrm{~ms}$, the differences between the elastic model and damage model have not been so pronounced, despite some differences in the force peak intensity.

From $7.0 \mathrm{~ms}$ on, the differences between both models were higher, and they behaved differently until the end of the impact event.

It is important to highlight how the orthotropic degree affects the behavior of cylinders and its damage process as well. It can be observed that for cylinders type A, the damage effects on the force response are not very much pronounced when compared to cylinders type $\mathrm{B}$ and $\mathrm{C}$ responses. 


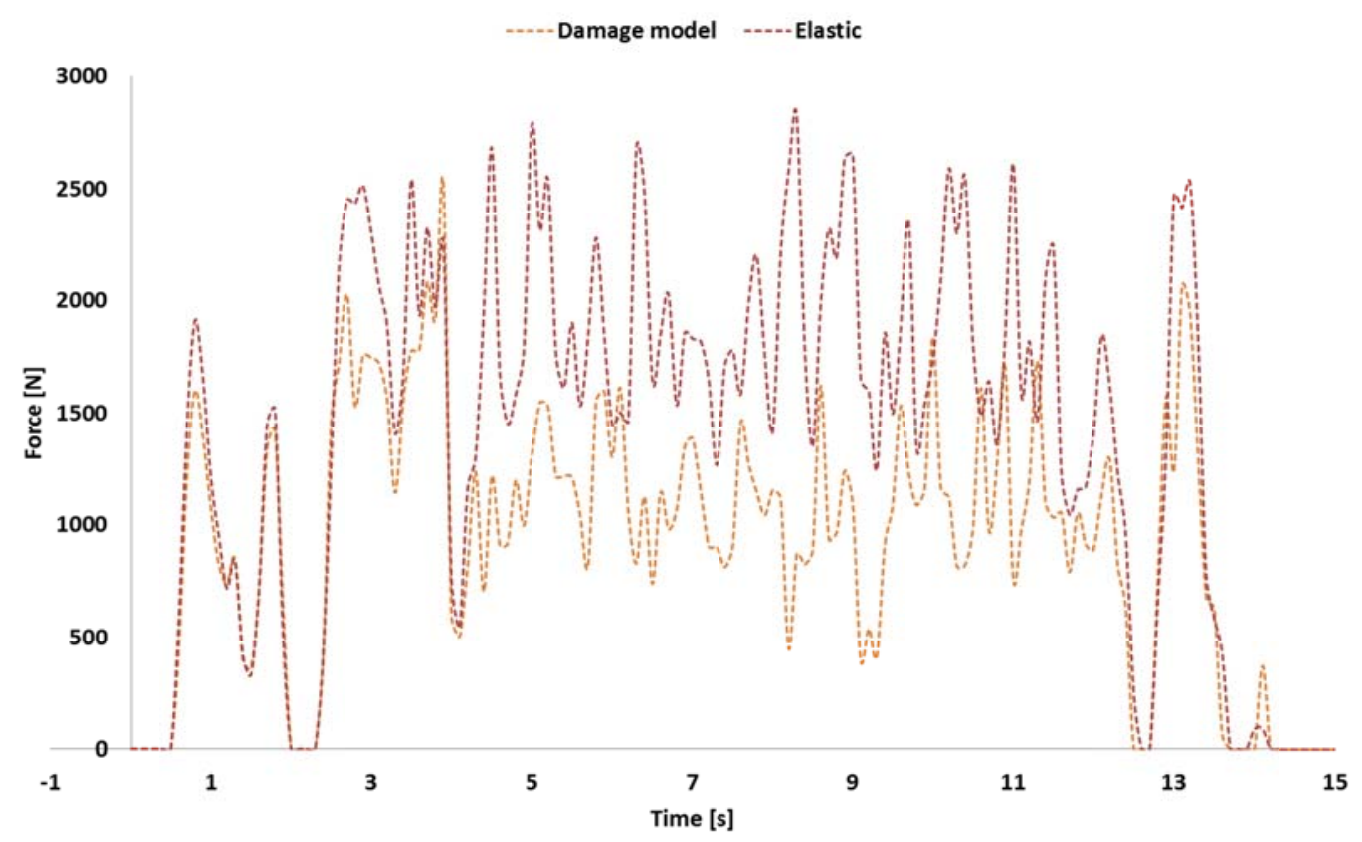

Figure 109: Type C cylinders: Elastic Model vs. Damage Model results (without damping effects).

\subsubsection{Results}

Considering the sensitivity studies performed in the previous sections, "the best" cylinder configurations for each cylinder lay-up are summarized in Table 45. The most refined mesh (Figure $87(\mathrm{c})$ ) has been used in the numerical simulations.

Table 45: FE model summary.

\begin{tabular}{ccccc}
\hline $\begin{array}{c}\text { Cylinder } \\
\text { Type }\end{array}$ & Normal Behavior & Tangential Behavior & $\begin{array}{c}\text { Contact } \\
\text { Algorithm }\end{array}$ & Damping $(\alpha)$ \\
\hline $\begin{array}{c}\text { Type A, B } \\
\text { and C }\end{array}$ & Hard Contact & $\begin{array}{c}\text { Penalty Method Friction } \\
\text { coefficient }=0.3\end{array}$ & General & 1.0 \\
\hline
\end{tabular}

The evaluation of the FE models regards the criteria that were already described, i.e., the force peaks intensity, the time of the force peaks, frequency of oscillations and visual appearance of the FE results.

\subsection{Results for Type A cylinders}

Despite a higher damping parameter ( $\alpha \geq 1000$ ), which better simulates the end part of an impact, these values affect the damage variables significantly (Figure 93, Figure 94 and 
Figure 95). Since the fiber damage is very important, regarding the composite structural integrity, the absence of fiber damage detection is regarded not to be acceptable for this work. Thus, lower damping values must be used to avoid underestimation of the damage.

Furthermore, once most of damage did not happen in the end of the numerical simulations, the accuracy to reproduce the end part of the impact (from $12.5 \mathrm{~ms}$ - Figure 110) has been considered to be relatively less important than from the beginning to $12.5 \mathrm{~ms}$.

The FE model parameter and experimental test results for the force vs. time are shown in Figure 110. A more refined mesh improved the overall model performance to simulate the impact force behavior. Thus FE force peaks, time when these peaks occur and force oscillations were more similar to the experiments data, regardless the final part of the impact (from $12.5 \mathrm{~ms}$ ). Despite the model performance improvement at $6.0 \mathrm{~ms}$, the experimental forces showed a high force peak but the simulated force was null at that time. Another remarkable difference happened at $8.0 \mathrm{~ms}$, where the simulated force was around $2000 \mathrm{~N}$ and the experimental force was around $1200 \mathrm{~N}$. At this point, the model detected the fiber failure onset. On the other hand, from $8.5 \mathrm{~ms}$ to $14.0 \mathrm{~ms}$, the numerical simulation predicted the force history with a reasonable accuracy. From $14.5 \mathrm{~ms}$ on, the force dropped to $0 \mathrm{~N}$, then increased again up to $1500 \mathrm{~N}$ and, finally, decreased to $0 \mathrm{~N}$. This behavior was rather different from the experiments, which showed a much smoother behavior.

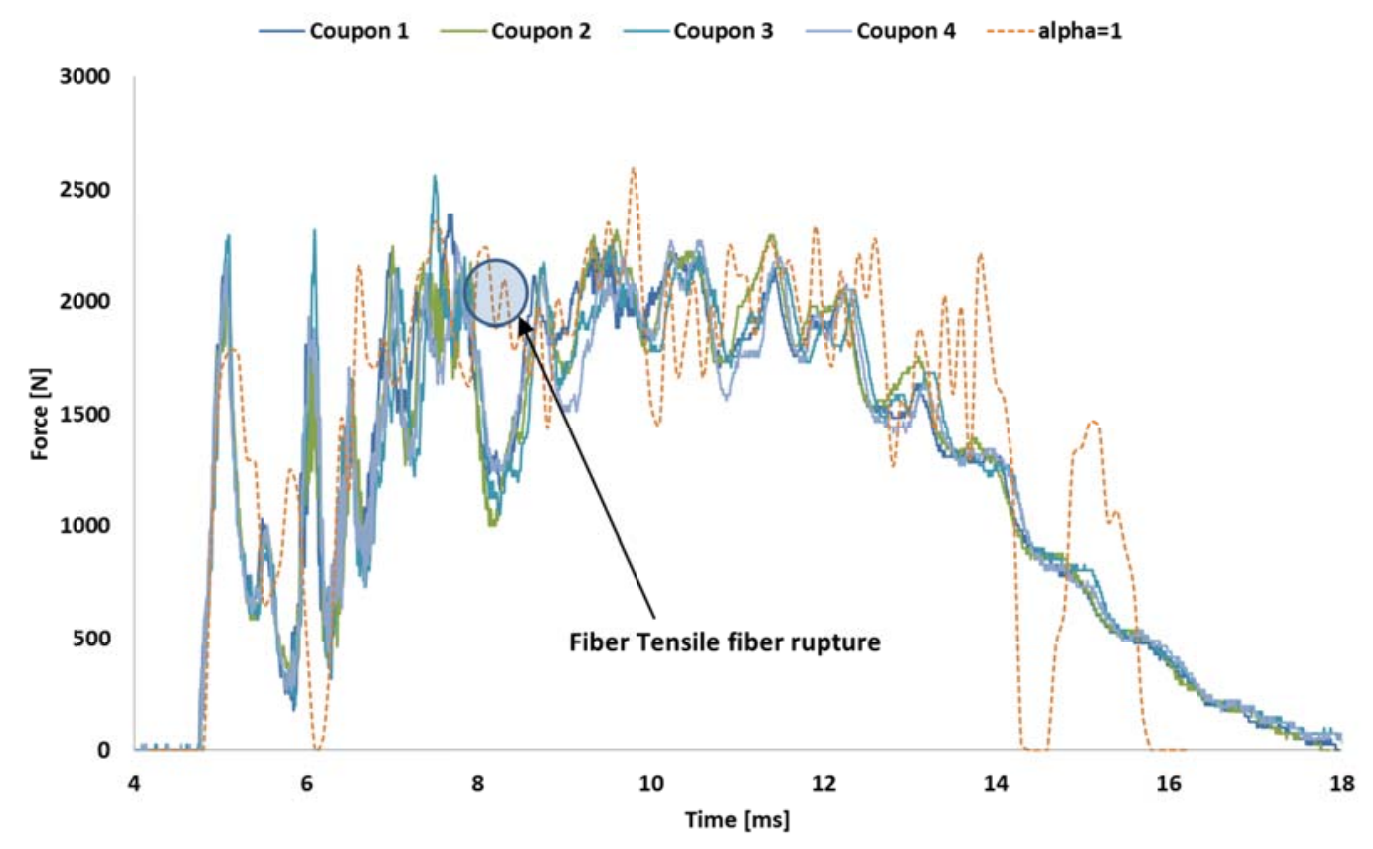

Figure 110: Type A simulation results. 
Moreover, when $d_{1}$ was detected, it did not correspond to the highest force peak on the impactor head, despite $\sigma_{11}$ being very high (Figure 111) from $0.4 \mathrm{~mm}$ to $0.6 \mathrm{~mm}$ but, from $2.8 \mathrm{~mm}$ to $3.5 \mathrm{~mm}$, it was observed to be a high compressive $\sigma_{11}$.

It is important to highlight that for compressive $\sigma_{11}, \mathrm{~d}_{1}$ is not defined, but $\mathrm{E}_{11}$ decreases following the secant modulus as established by the EQ. (15).

Regarding the FE simulation for force vs. time shown in Figure 110, the simulations results for final part (from $14.0 \mathrm{~ms}$ on) have not been good. This difference was due to the dynamics of the cylinder displacement, where the cylinder surface lost the contact with the impactor head. After few seconds (less $0.3 \mathrm{~ms}$ ), the cylinder surface touched the impactor again creating a force peak (the simulation's last one). After this peak, the cylinder surface displacement stopped for few seconds, losing again its contact with the impactor head. From this time, the cylinder surface and the impactor head have not interactedl anymore.

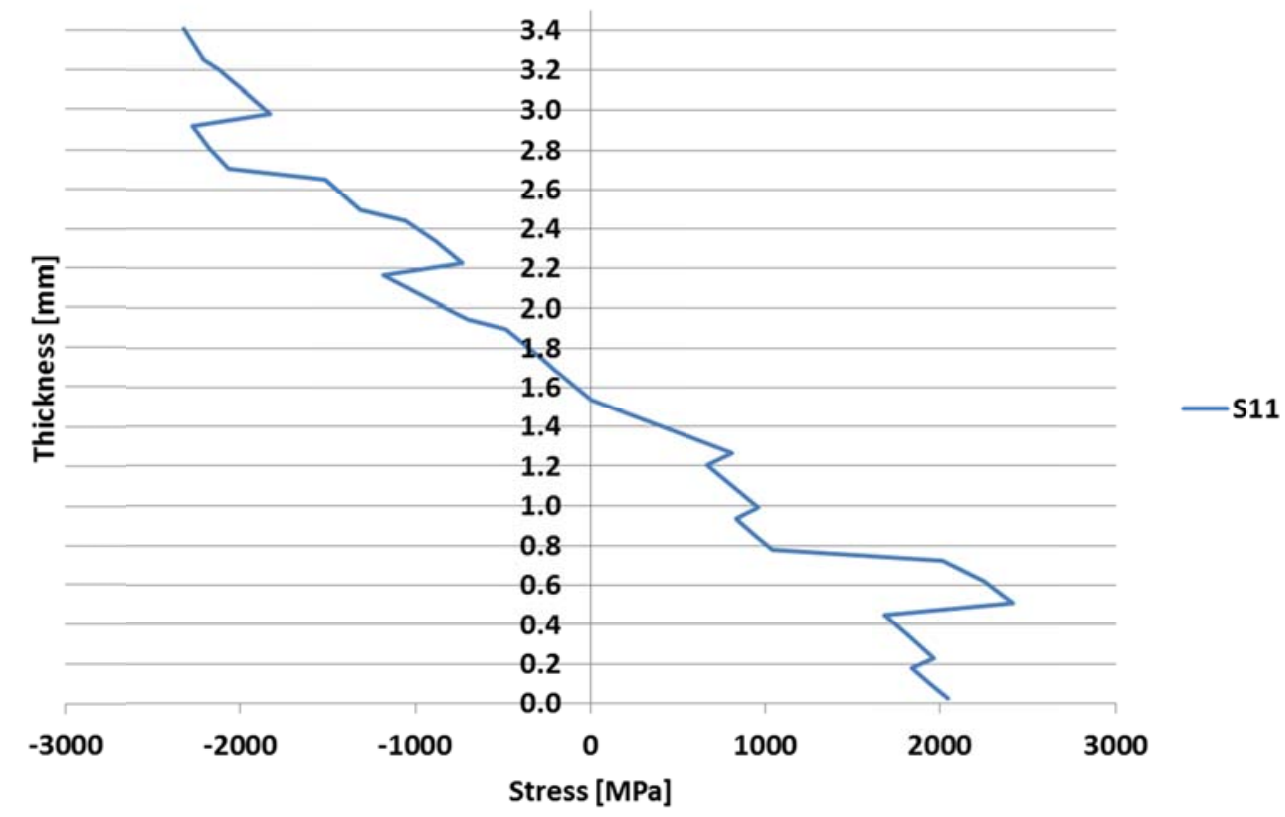

Figure 111: Type A $\sigma_{11}$ Stress (S11) at fiber failure

The transverse stress, $\sigma_{22}$, through the thickness at fiber failure showed onset maximum value around $40 \mathrm{MPa}$ (between $0.4 \mathrm{~mm}$ to $0.6 \mathrm{~mm}$ ) as observed in Figure 112. The compressive $\sigma_{22}$ was around $-30 \mathrm{MPa}$ from $0.2 \mathrm{~mm}$ to $0.4 \mathrm{~mm}$ and, from $2.8 \mathrm{~mm}$ to $3.0 \mathrm{~mm}$. Compressive $\sigma_{22}$ did not trigger the matrix damage variable $\mathrm{d}_{2}$ and, the elastic modulus, $\mathrm{E}_{22}$ 
decreases as defined by EQ.(22). It is important to notice that the layers closer to laminate surfaces have been more loaded than layers closer to the laminate middle plane.

It is worth to remember that through the thickness, the stress distribution pattern has been calculated in local coordinates by using the Classical Laminate Theory. Thus, the stress distribution depends on the ply orientation as well as on its distance from the middle plane.

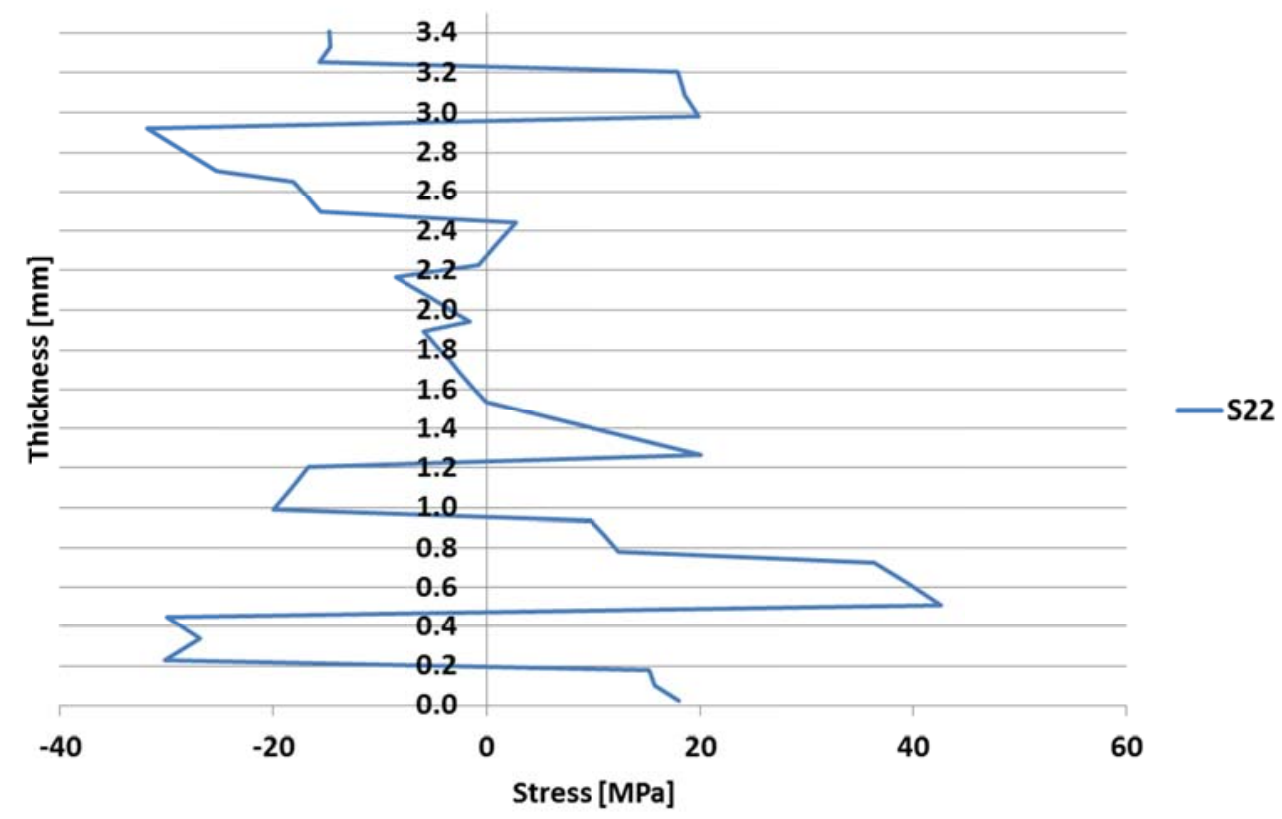

Figure 112: Type A $\sigma_{22}$ Stress (S22) at fiber failure

The shear stress distribution through the thickness is shown in Figure 113. This figure shows that the shear stress increased from the middle plane to the external layers. Also, shear stress has been considerably higher than transverse stress (around ten times higher). 


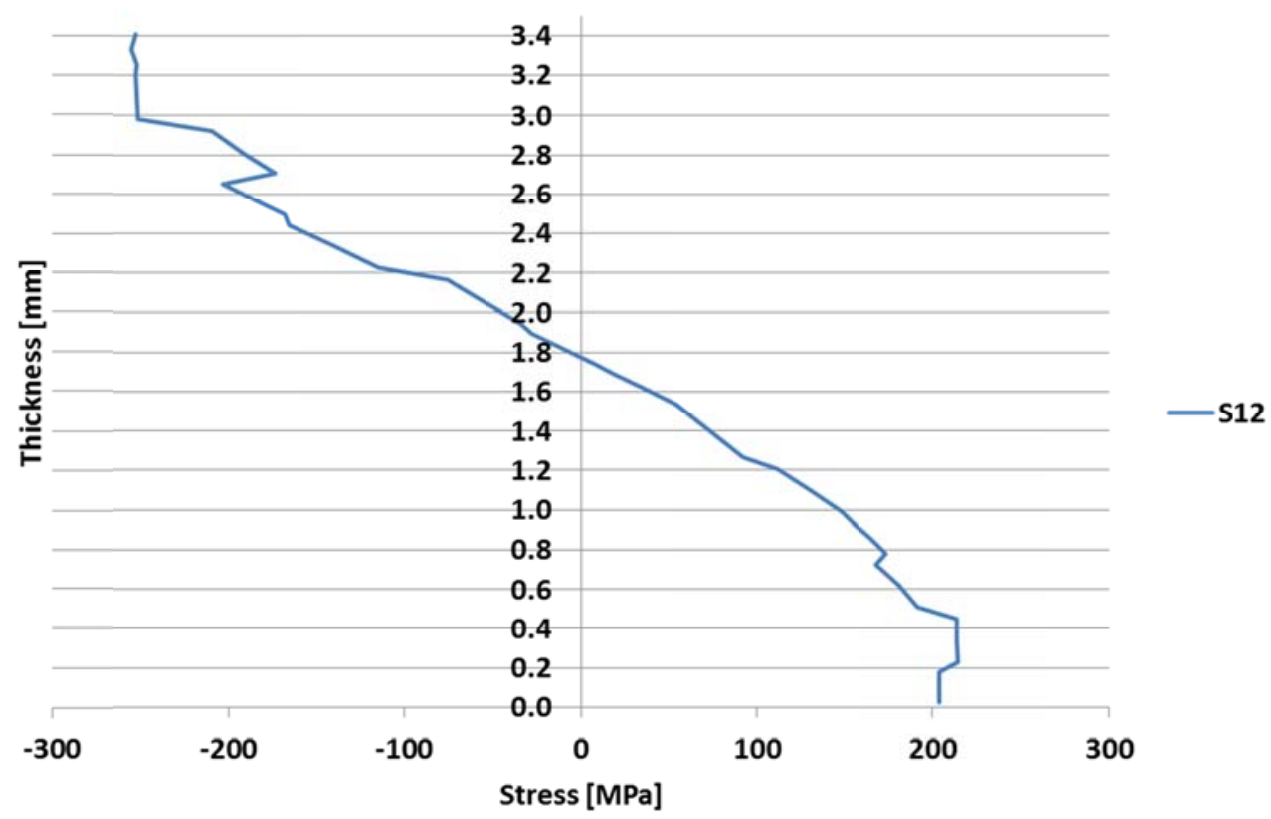

Figure 113: Type A $\tau_{12}$ Stress (S12) at fiber failure.

From Figure 112 and Figure 113, it is possible to conclude that the external layers have been more damaged than the internal ones, once the stress state in the external layers are considerably higher than that in layers closer to the laminate middle plane.

To improve the understanding of the fiber damage through the thickness, the following figures show the damage extension layer- by- layer. The impactor head as well as the layup are also shown to aid the comprehension of the results. 


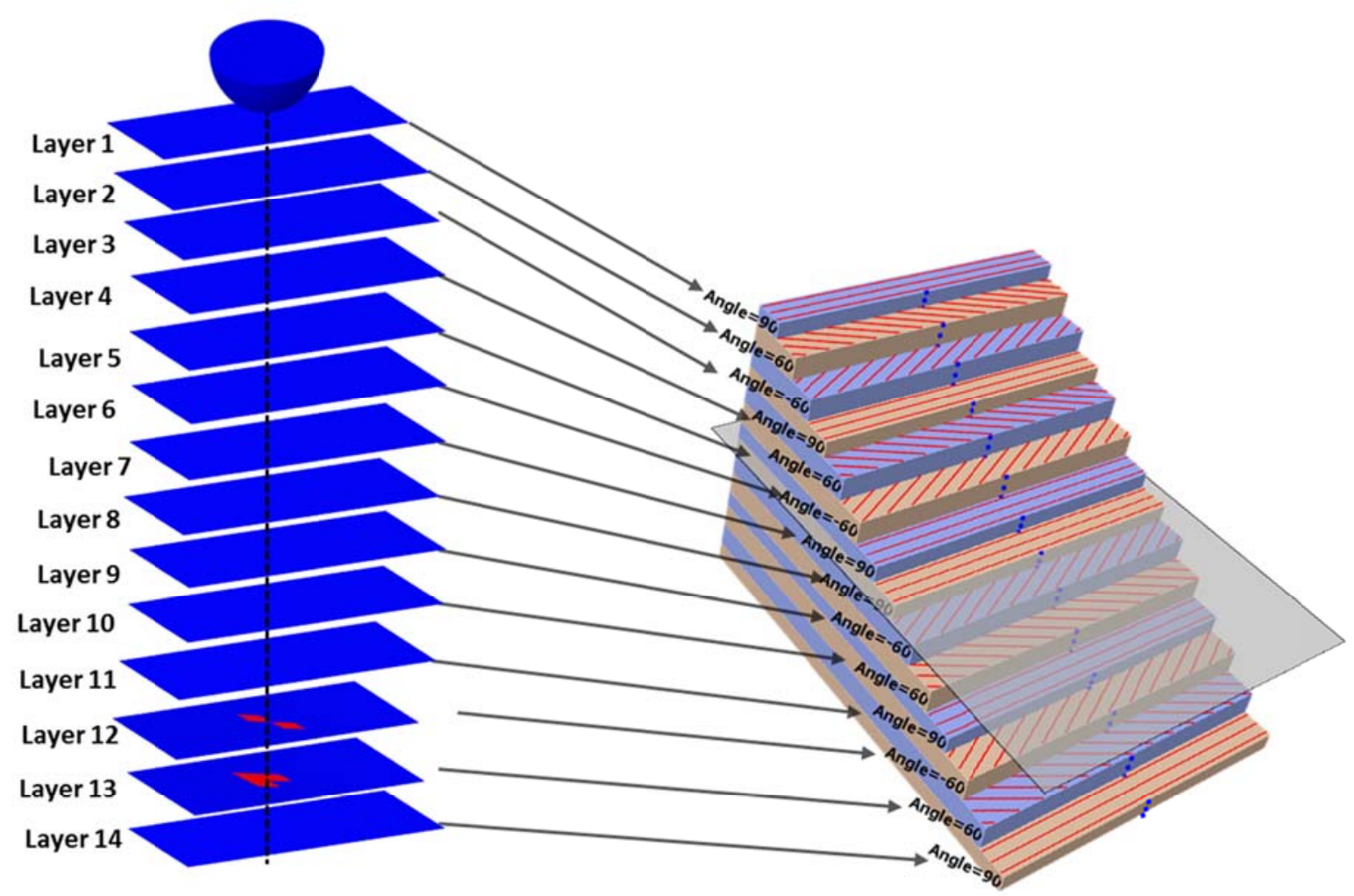

Figure 114: Type A: $d_{1}$ damage through the thickness

Figure 114 represents the fiber damage layer- by- layer after the impact, accounting the total damage calculation. It can be observed that fiber failed at layer 13 and 12 with orientation $60^{\circ}$ and $-60^{\circ}$. No fiber damage has been detected neither in layer 14 nor in the other layers. This damage calculation can explain why this damage has not been detected by visual inspection on the cylinders.

For a complete overview of matrix damage, Figure 115 and Figure 116 must be analyzed together. In layer 1, the damage intensity is high, but its extension is smaller than the $\mathrm{d}_{2}$ in layer 14 (Figure 115) and $d_{6}$ in layer 5 (Figure 116). Furthermore, the high intensity damaged area decreased from the external layers towards the laminate middle plane. For $\mathrm{d}_{2}$, damage has not been detected in layers 7 and 8 (Figure 115). On the other hand, the matrix in these layers have been basically damaged due to shear stress (Figure 116). 


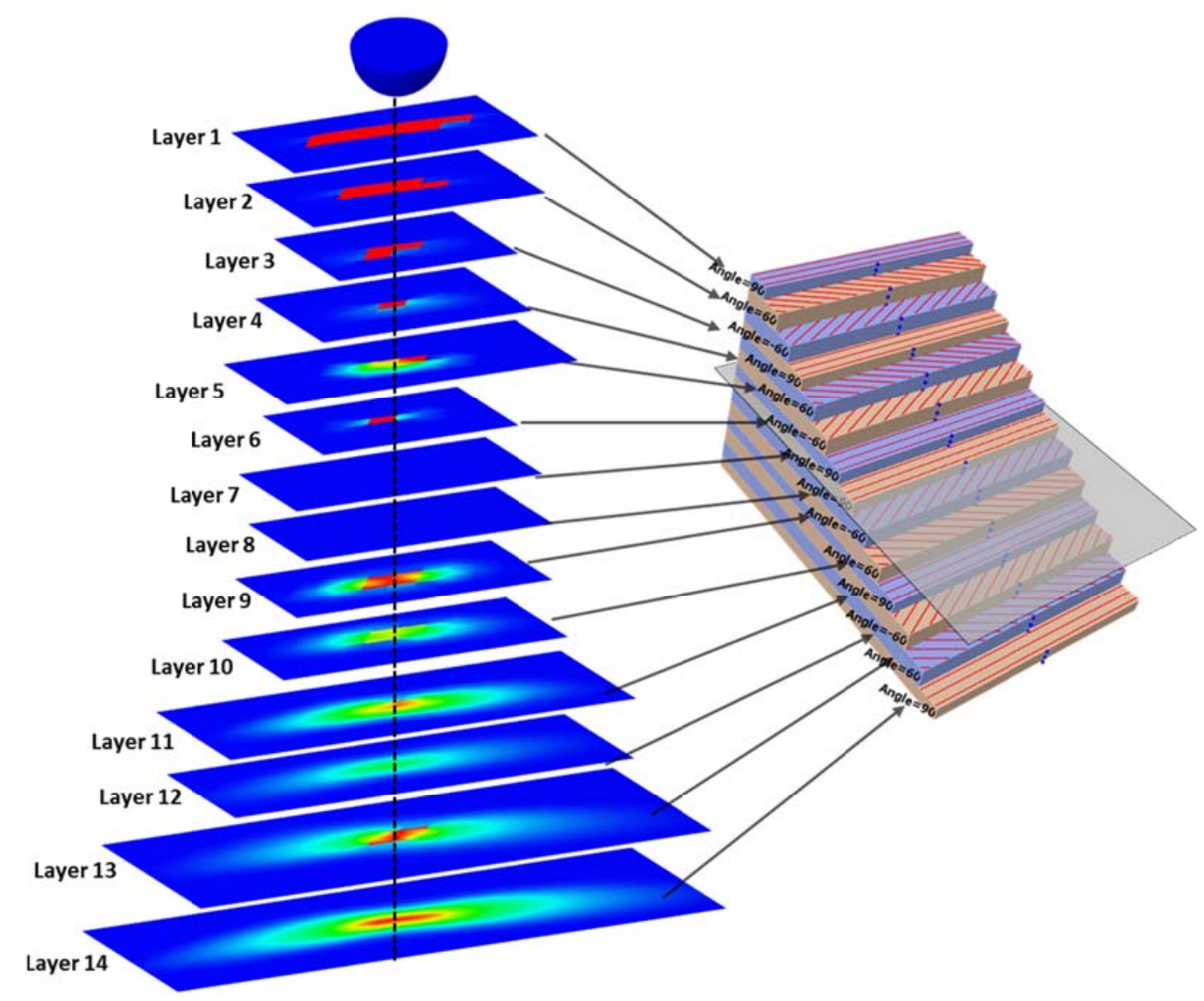

Figure 115: Type A: $d_{2}$ damage through the thickness

The extension of $d_{2}$ in layer 14 has been rather higher than in the other layers, but its intensity has been only closer to the impact line (dot line in the Figure 115). Also, the shear damage has been more pronounced in this layer. Thus, the matrix has been completely damaged in the external layers. From Figure 116, it can be observed that layer 12 did not have damage due to shear stress, but layer 12 showed some damage due to $\mathrm{d}_{2}$.

Moreover, for type A cylinders, all layers had the damaged matrix, once layer 7 and 8 did not show $d_{2}$ damage, but they showed $d_{6}$ damage as well as in the layer 12 , where $d_{6}$ damage was not shown, but $\mathrm{d}_{2}$ damage was. 


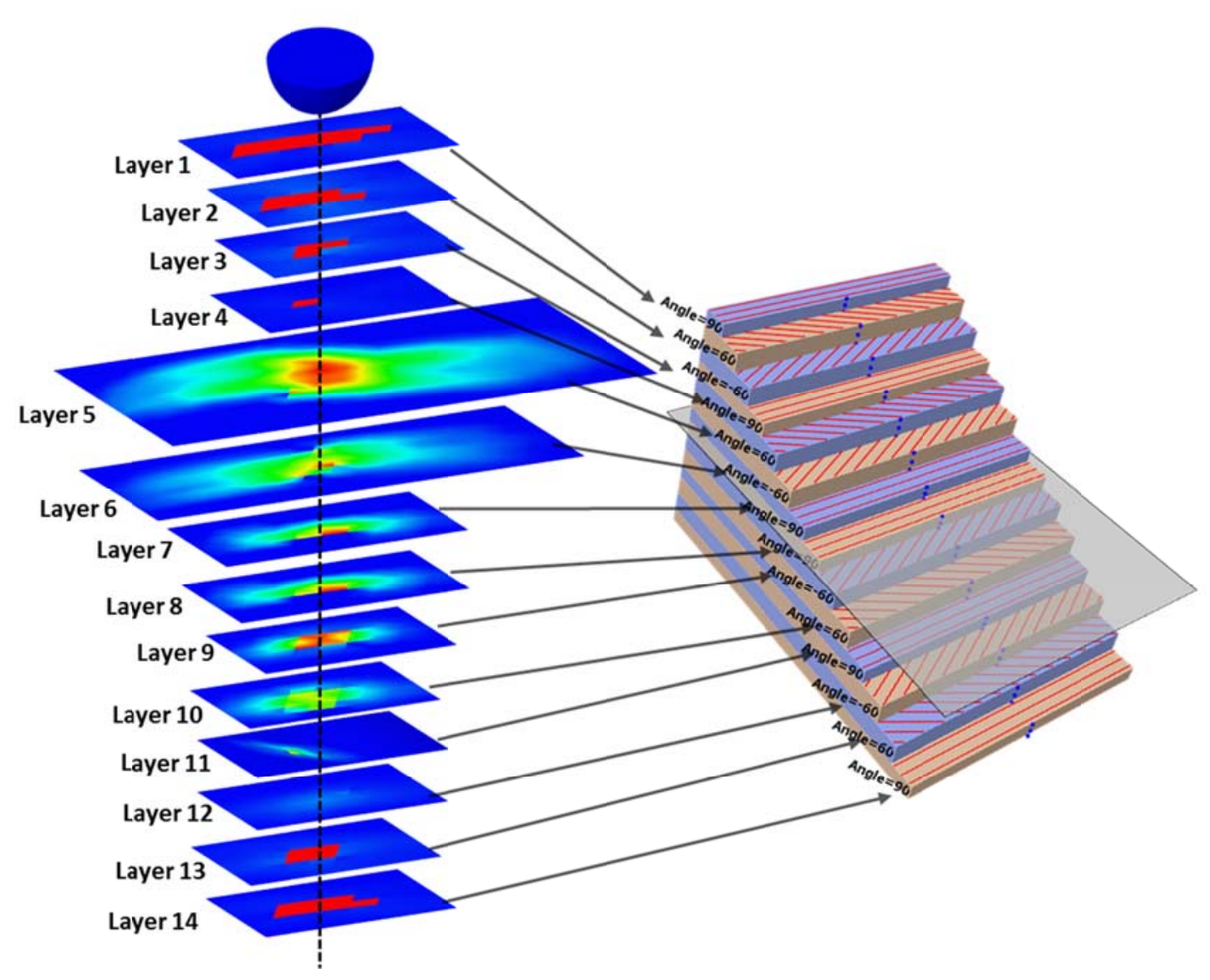

Figure 116: Type A: $d_{6}$ damage through the thickness

Figure 117 shows the total damage variables through the thickness for the most critical element, i.e. closer to the impactor head (Figure 92). As mentioned earlier, the external layers had more matrix damage and, the damage decreased towards the laminate middle plane. This figure shows that each layer had at least matrix damage, i.e. the $31 \mathrm{~J}$ impact showed damages in all layers.

An important conclusion based on the analysis of all those figures is that the damage pine tree shape for impacted laminates has not been found for those numerical simulations. More investigations on the coupons should be performed to confirm this phenomenon by using layer- by- layer analysis. 


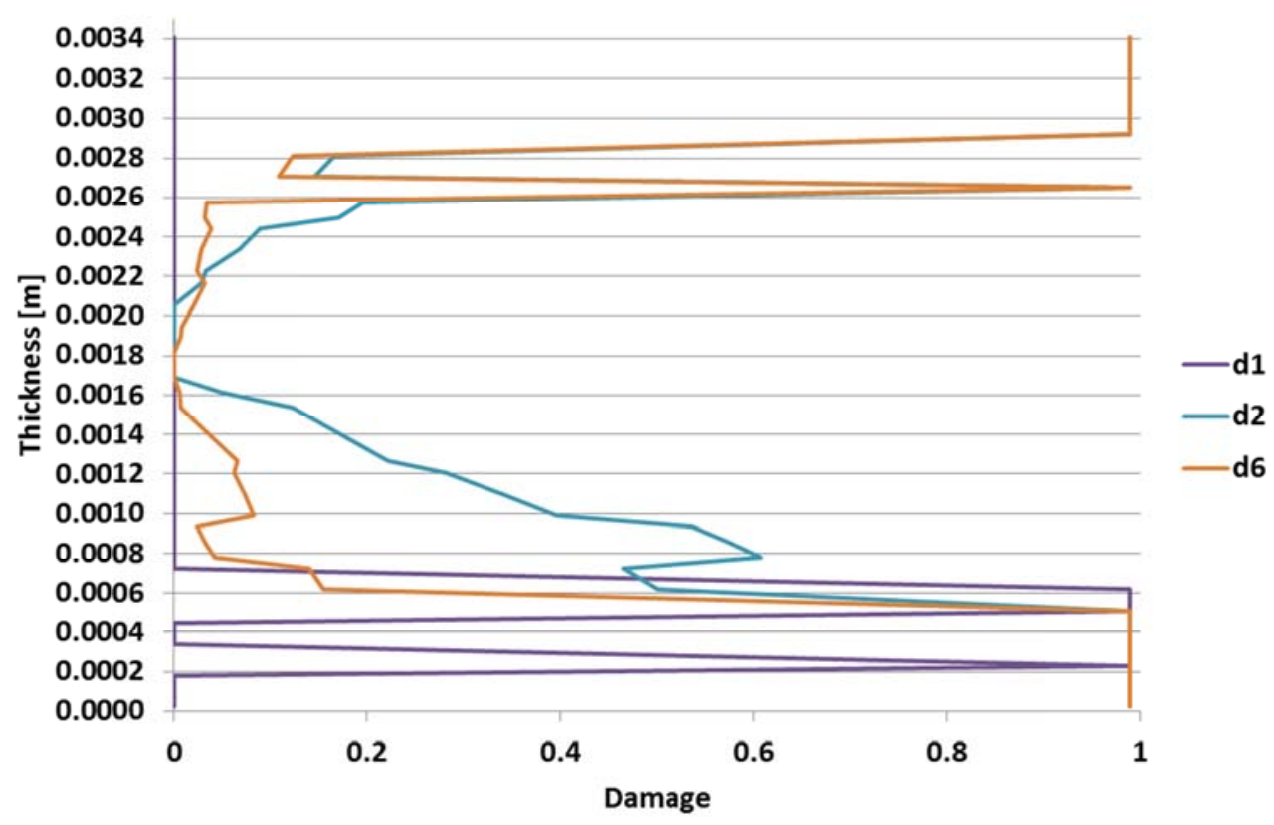

Figure 117: Damage variables for the impact point (total time)

\subsection{Results for Type B cylinders}

As mentioned before, higher damping parameters made the FE models provide underestimation results for fiber damage (Figure 98). Once fiber failure is a very important failure mode, this lack of fiber damage detection has not been acceptable; so lower $\alpha$ values must be used in order to avoid not only the underestimation of fiber damage, but also the FE model performance improvement.

Since most of the damage process has happened until $13.0 \mathrm{~ms}$ for cylinders type $\mathrm{B}$, the final part (from $13.0 \mathrm{~ms}$ on) did not have the same importance as the interval time from 4.7 $\mathrm{ms}$ to $13.0 \mathrm{~ms}$ (Figure 118).

Some high load peaks at the beginning of the impact (around $6.7 \mathrm{~ms}$ ) have not been simulated. For example, at $5.4 \mathrm{~ms}$, the experimental forces showed a peak, but the numerical forces were equal to null at this point. After that, the numerical forces increased again as the experimental forces $(6.5 \mathrm{~ms})$, but the experiments have formed the highest overall value for force (at $6.7 \mathrm{~ms}$ ). At this time, the FE model had shown only a force peak, but not so pronounced. Despite this divergence between FE model and experiments at the beginning of the impact response, the overall behavior has been acceptable.

Other discrepancy between experiments and FE model happened at $7.5 \mathrm{~ms}$, when the experiments showed a peak and, the FE model presented a valley. From $8.0 \mathrm{~ms}$ s to $13.0 \mathrm{~ms}$, the numerical force showed a behavior similar to the experimental force. 
Another important aspect was that the highest load peak measured in the impactor head did not correspond necessarily, to the highest laminate stress state (plane stress). The highest stress state happened when fiber failed at $11.0 \mathrm{~ms}$, i.e. after $5.0 \mathrm{~ms}$ from the highest load peak. On the other hand, the fiber failed at the larger displacement of the cylinder surface in contact with the impactor head, which yields a stress state strong enough to trigger the fiber failure.

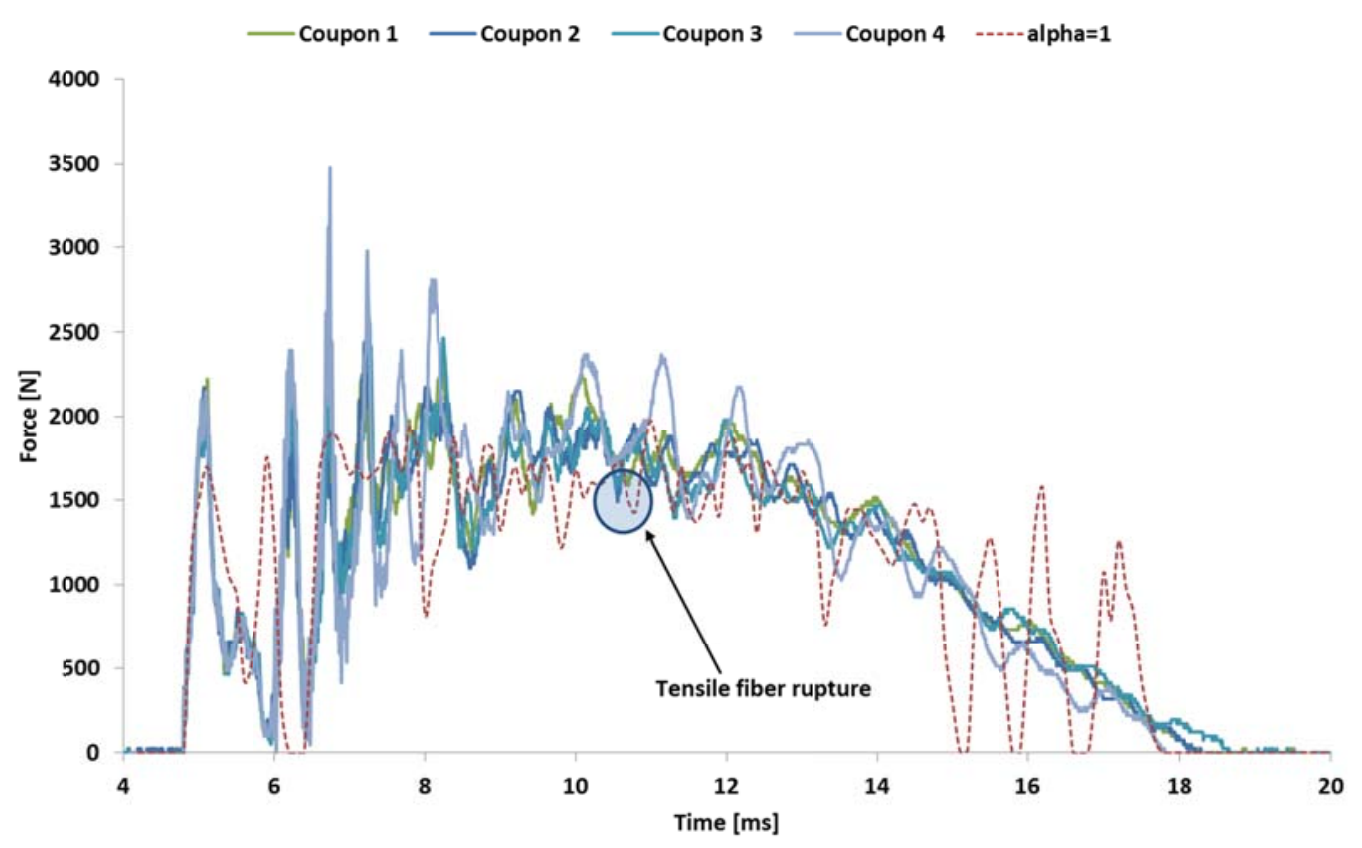

Figure 118: Type B results.

For the impact final part (from $14.7 \mathrm{~ms}$ on), the simulation diverged from the experiments as already verified in the previous cases.

The stress state through the thickness at fiber failure onset is presented in the following figures. Figure 119 shows the results for $\sigma_{11}$. It is observed that the external layer at $0.0 \mathrm{~mm}$ had the biggest $\sigma_{11}$ tensile value. In the opposite external layer (at $3.5 \mathrm{~mm}$ ), the compressive stress is not higher than the stress at $3.0 \mathrm{~mm}$. Once again, it is important to mention that $\mathrm{d}_{1}$ has not been calculated for compression stress $\sigma_{11}$, despite $\mathrm{E}_{11}$ decreasing as established by the EQ.(15).

The analyses of transverse stress $\left(\sigma_{22}\right)$ distribution through the thickness at fiber failure onset are shown in Figure 116. This stress distribution reached the highest value at $2.8 \mathrm{~mm}$, where $\sigma_{22}=18 \mathrm{MPa}$. 


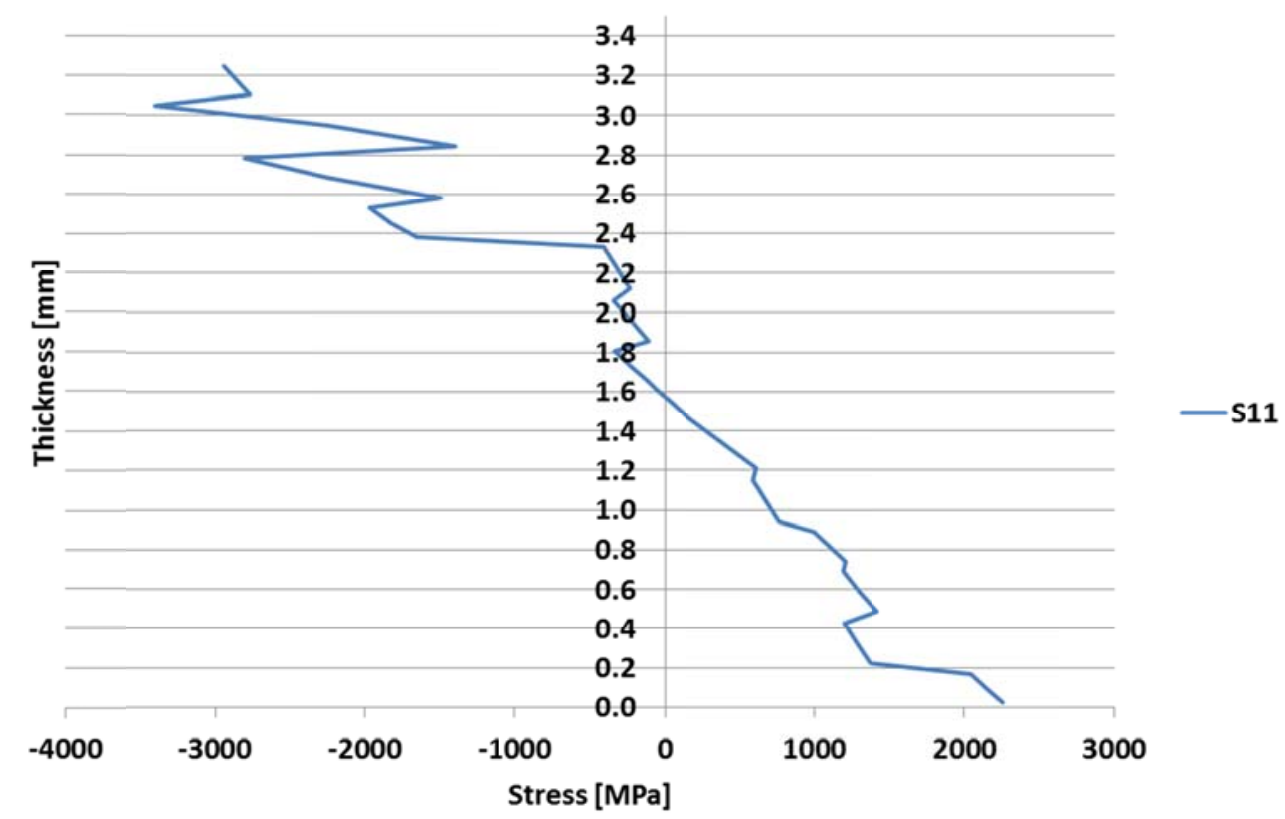

Figure 119: Type B $\sigma_{11}$ Stress (S11) at fiber failure.

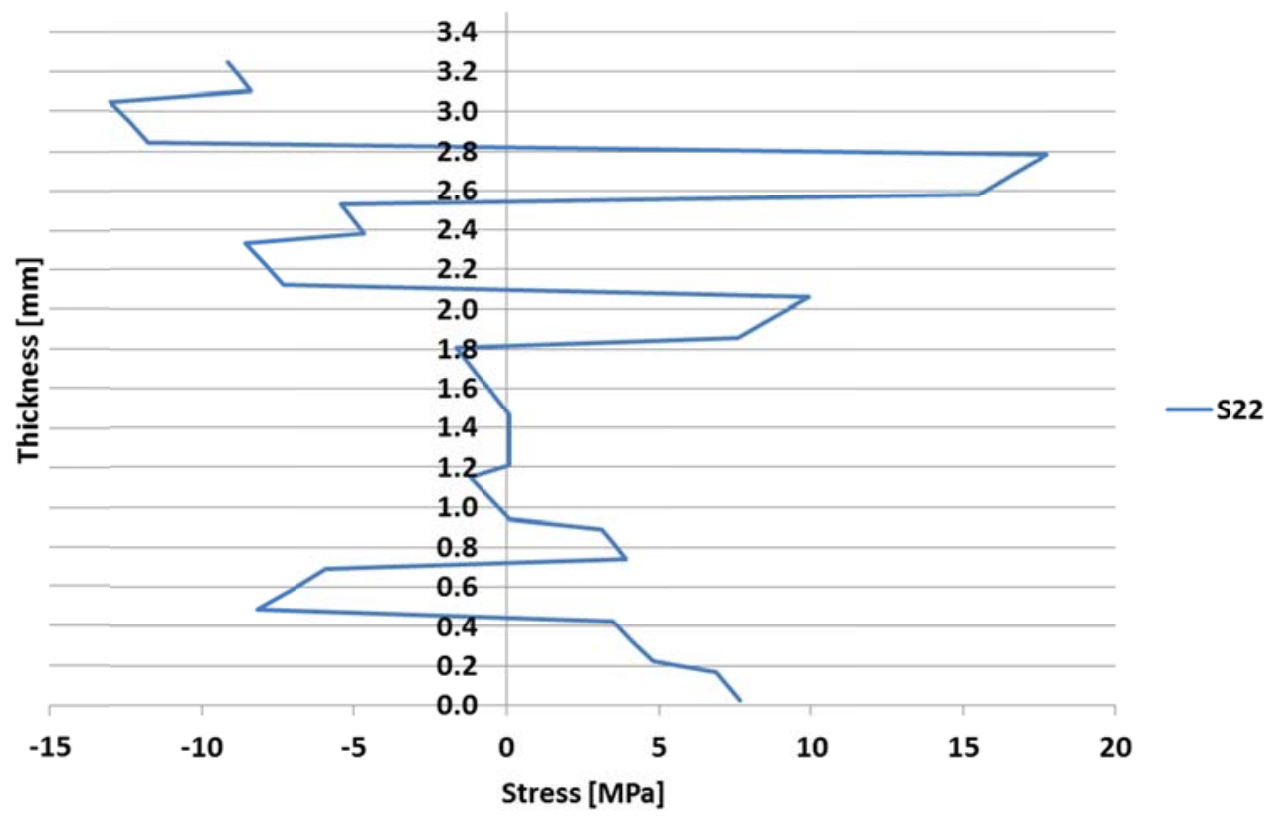

Figure 120: Type B $\sigma_{22}$ Stress (S22) at fiber failure.

Moreover, layers from $2.6 \mathrm{~mm}$ to $3.0 \mathrm{~mm}$ have been more loaded than the external layers and observing the shear stress through the thickness (Figure 121), matrix had its highest stress state (plane stress) at fiber failure onset in layer at $3.0 \mathrm{~mm}$. 


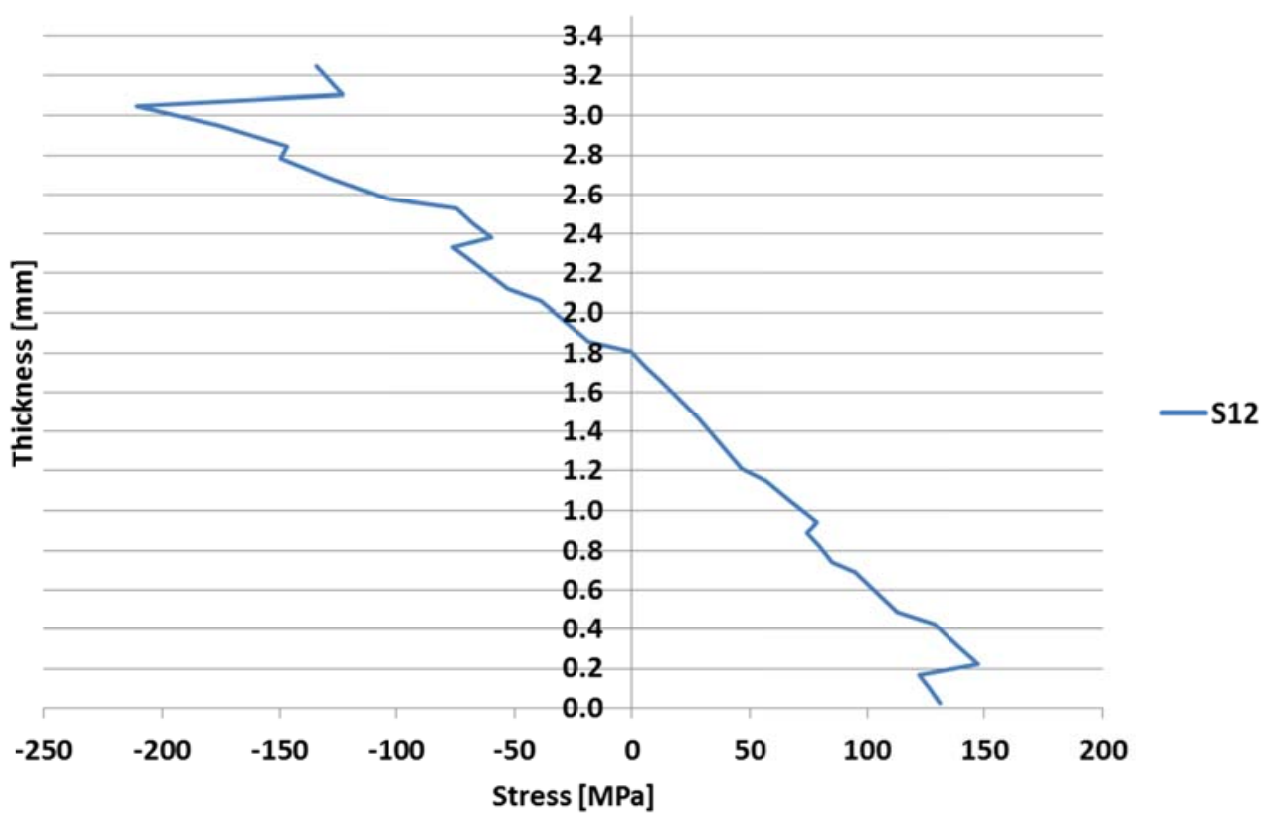

Figure 121: Type B $\tau_{12}$ Stress (S12) at the moment of layer 14 fiber breakage

The following figures show the damage variables for each layer after the impact simulation. These figures improve the visualization of damage for each layer. The composite lay-up is also shown and the orientation for each layer is indicated. In addition, the impactor head and its central line have also been represented.

Computational analyses of cylinders type B have shown fiber damage only in layer 14, i.e. the cylinder inner layer (Figure 122). The damage extension is small, only few elements have been damaged. Despite the FE model having shown fiber damage in the inner layer, coupons visual inspection did not detect fiber damage at this position. 


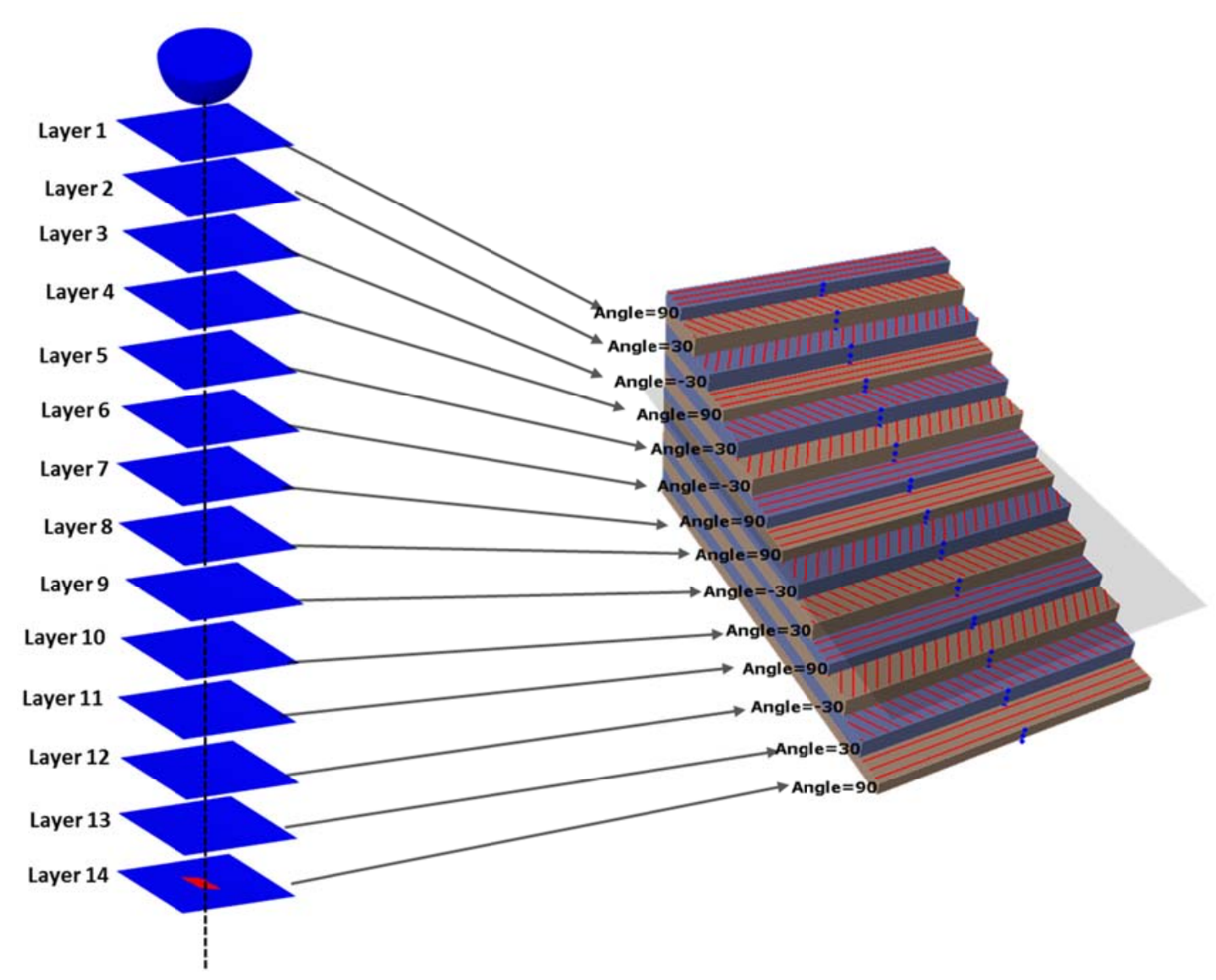

Figure 122: Type B: $d_{1}$ damage through the thickness

To identify the matrix damage, Figure 123 and Figure 124 must be analyzed together. At this lay-up, the outer ply (where the impactor touches the cylinder) has seemed to be damaged, but with less intensity than in the cylinder inner layer. Also, the damage was more localized and only few elements were completely damaged by $d_{2}$. On the other hand, $d_{6}$ was more spread than $\mathrm{d}_{2}$, but with lower intensity.

In layers 2 and 3, the matrix damage intensity increased and spread over more elements as well. From layers 4 to 6 , the damage intensity and the damaged area decreased and, in layer 7 , there was neither $\mathrm{d}_{2}$ nor $\mathrm{d}_{6}$ detection.

From layer 8 , the matrix damage intensity and affected area increased again. Since the damage intensity increased, the damaged area also increased, but the maximum matrix damaged area did not correspond to the highest matrix damage intensity. For example, the damage intensity on matrix was the highest in layer 14 , where $\mathrm{d}_{2}$ had its highest value (Figure 123), but the matrix damage was the most spread in layer 13 , where $d_{6}$ was the most distributed (Figure 124). 


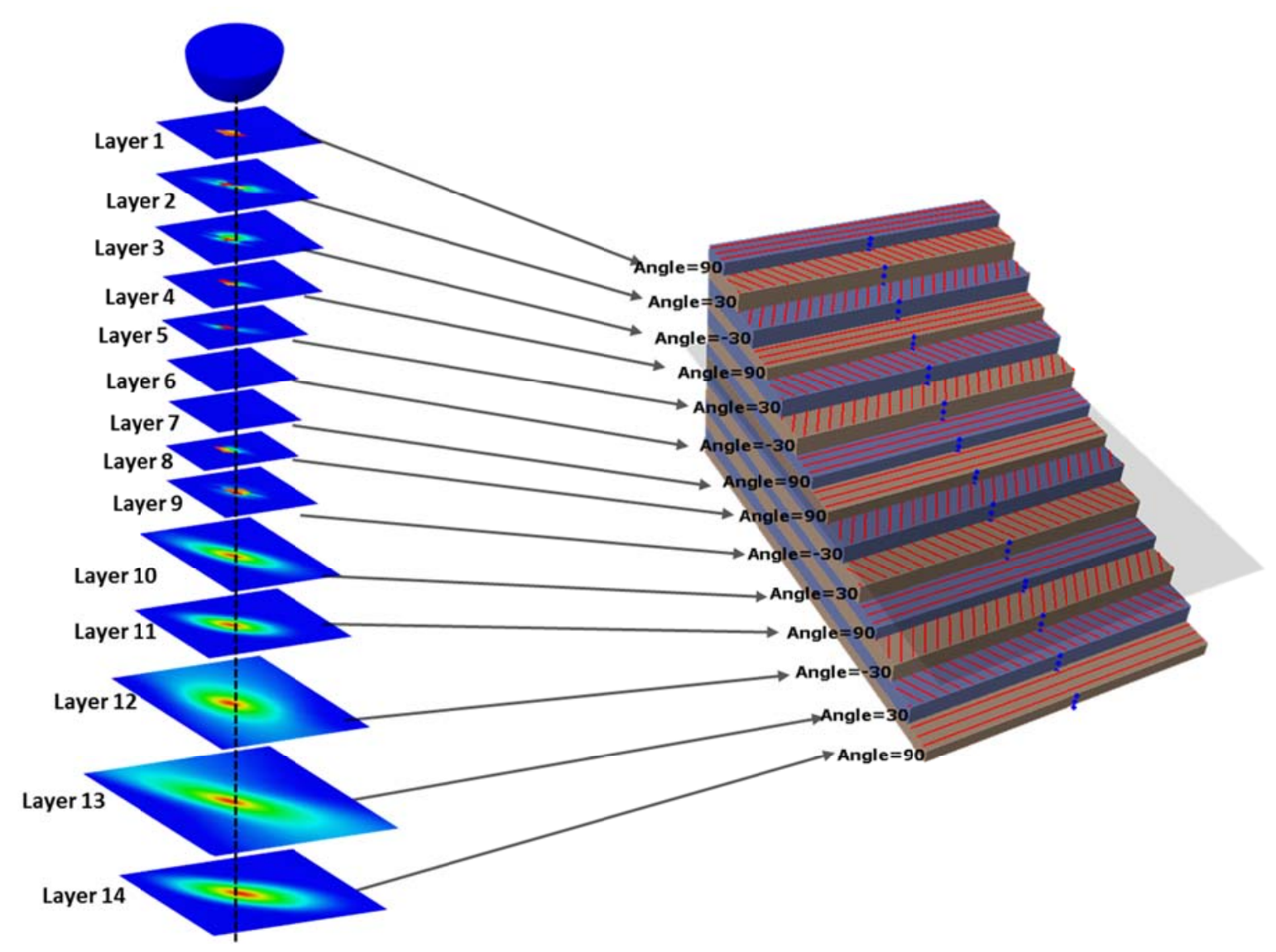

Figure 123: Type $B: d_{2}$ damage through the thickness

Regarding the relation between damage intensity and area, it can be observed that layer 13 and 2 had higher intensity for $\mathrm{d}_{6}$ as well as the highest affected area. The shear damage $\mathrm{d}_{6}$ has been more spread than $d_{2}$ for all layers. On the other hand, $d_{2}$ has been more intense than $d_{6}$.

Furthermore, $d_{2}$ has not been detected in two layers (layer 6 and 7 ) and, $d_{6}$ has not been detected only in layer 7. Once more, the pine tree shape has not been observed for those cylinders simulations. 


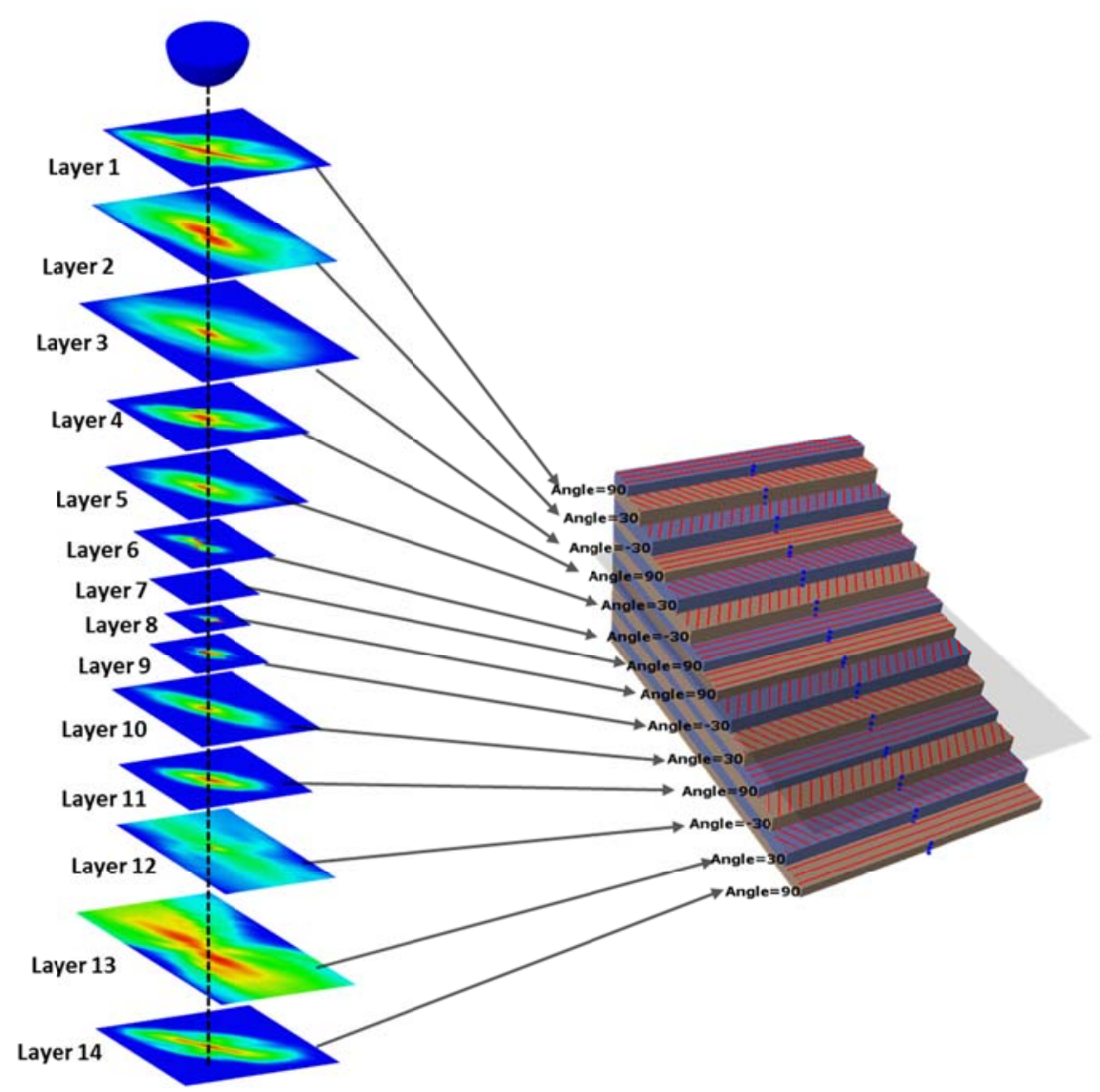

Figure 124: Type B: $d_{6}$ damage through the thickness

In order to show the amplitude of shear damage on type B cylinders, Figure 125 shows the whole cylinder overview of shear damage $d_{6}$ in layer 13. It can be observed that a considerable portion of the cylinder surface has been damaged. Despite the damage intensity being higher in the impact area the bottom of the cylinder has shown a considerable damage. The cylinder lateral area $\left(90^{\circ}\right.$ radial rotate from the impacted area - Figure 125 has also been damaged, but its intensity is lower than the other damaged areas. 


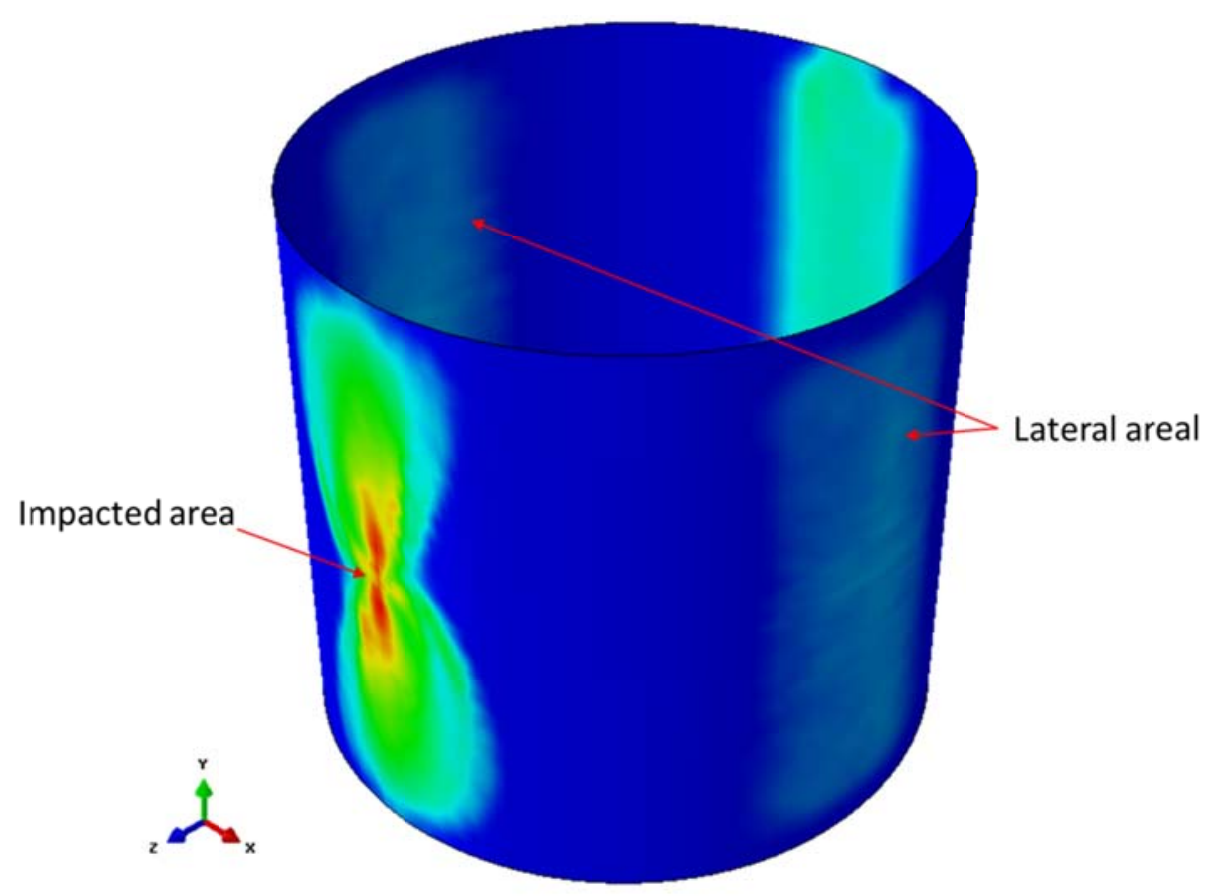

Figure 125: Layer 13 shear damage $\left(\mathrm{d}_{6}\right)$ on cylinder type B.

The damage analysis through the thickness on the most critical element is shown in Figure 126. This figure shows the damage state at the ending of the impact simulation.

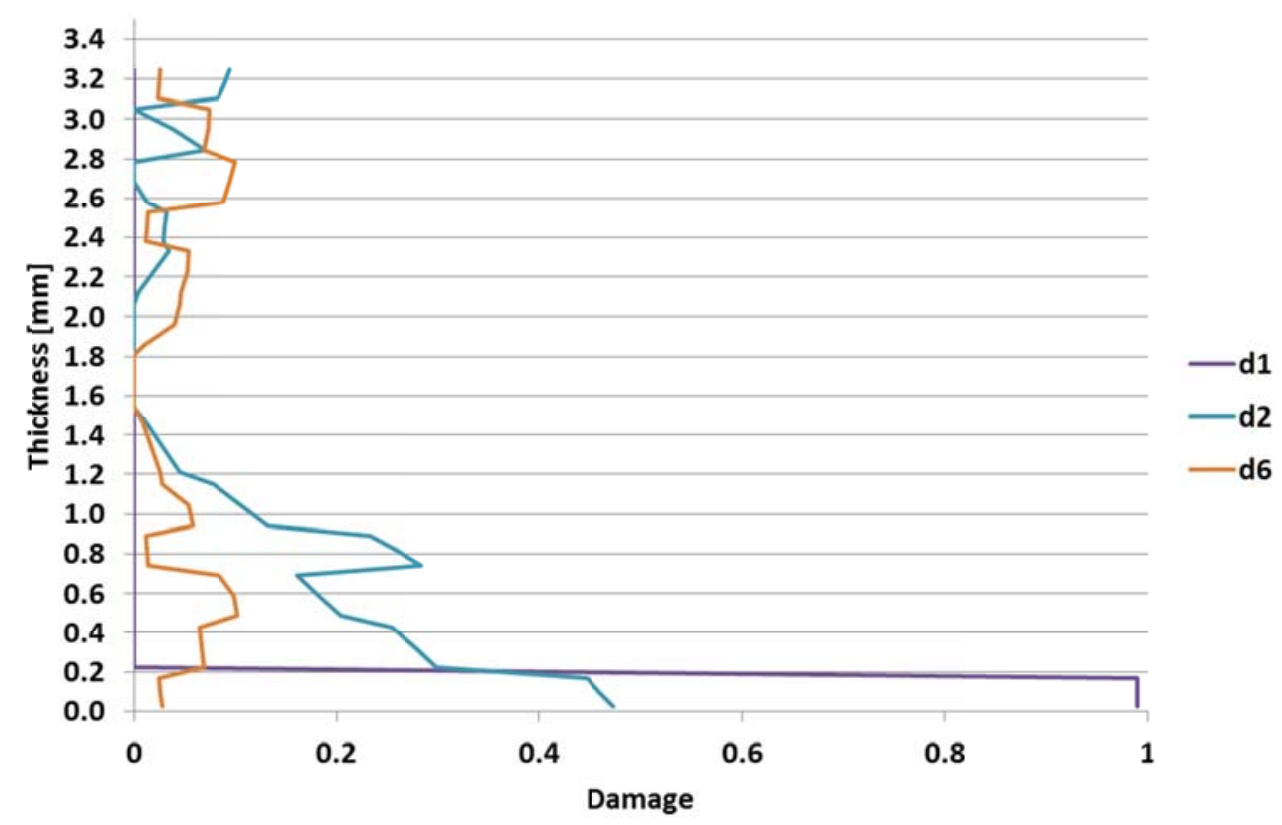

Figure 126: Type B damage variables through the thickness. 
As already shown, the fiber damage has only been detected in layer 14 (from $0.0 \mathrm{~mm}$ to $0.2 \mathrm{~mm}$ ). From this figure, it is observed that the matrix damage intensity has been considerably high in layer 14 , once $\mathrm{d}_{2}$ has the highest value. On the other hand, the shear damage in layer 14 has been low.

The shear damage has been higher from $0.45 \mathrm{~mm}$ to $0.65 \mathrm{~mm}$ and, from $2.6 \mathrm{~mm}$ to 2.8 $\mathrm{mm}$. Furthermore, the cylinder type B was more damaged in its inner layer. The cylinder had some matrix damage in the layer where the impactor touched, but it was smaller than that in layer 14 .

\subsection{Results for Type C cylinders}

The results for type $\mathrm{C}$ are presented in Figure 127. At the beginning of the impact, the FE model was capable to capture the first high load peak (at $4.8 \mathrm{~ms}$ ) with reasonable accuracy. Then, both the numerical and experimental force decreased to $500 \mathrm{~N}$ but at different time (5.0 $\mathrm{ms}$ for experiments and $5.3 \mathrm{~ms}$ for simulation). The simulated force increased again creating a second force peak and, the experimental force decreased to almost $0 \mathrm{~N}$.

After that, the simulated force dropped to $0 \mathrm{~N}$ but the experimental force increased showing a peak. The simulated force remained null and, the experimental force dropped to a value close to $0 \mathrm{~N}$. From this point, the force for both cases increased and, the experiments showed the highest force peak. At this point of the impact event $(6.7 \mathrm{~ms})$, the FE model resulted on a smoother pattern than the experimental data. 


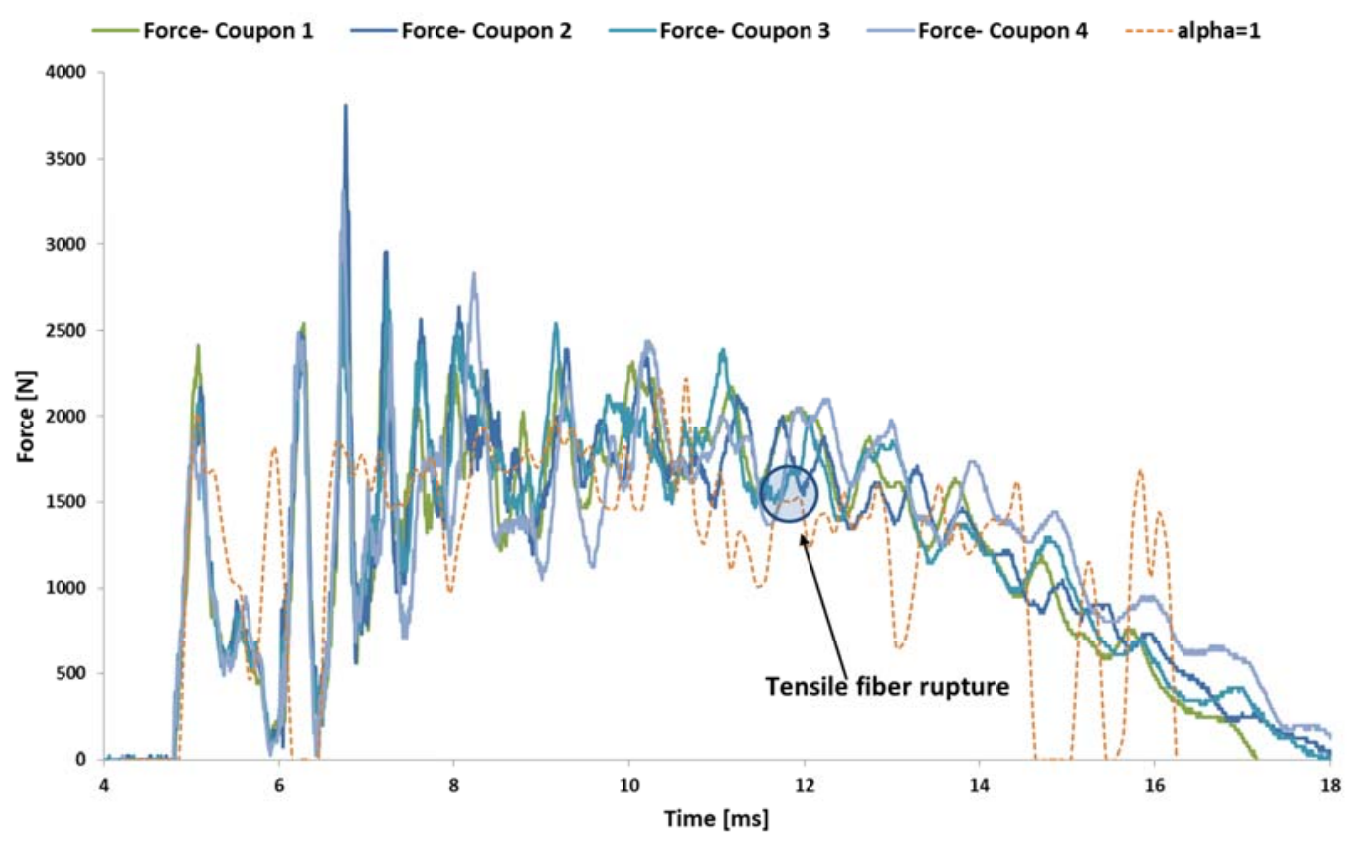

Figure 127: Type C results.

As for previous case, the final simulation part (from $14.7 \mathrm{~ms}$ on) diverged from the experiments. Regardless this fact, the overall simulation has been acceptable.

Figure 128 shows the $\sigma_{11}$ stress state at fiber failure onset for the FE most critical element. The highest tensile stress value has been detected at $0.0 \mathrm{~mm} . \sigma_{11}$ decreased and became null at $1.8 \mathrm{~mm}$, then it reached the lowest value at $3.4 \mathrm{~mm}$. It is important to mention that $\mathrm{d}_{1}$ is not calculated for compressive $\sigma_{11}$, despite $\mathrm{E}_{11}$ decreasing as described by EQ.(15). 


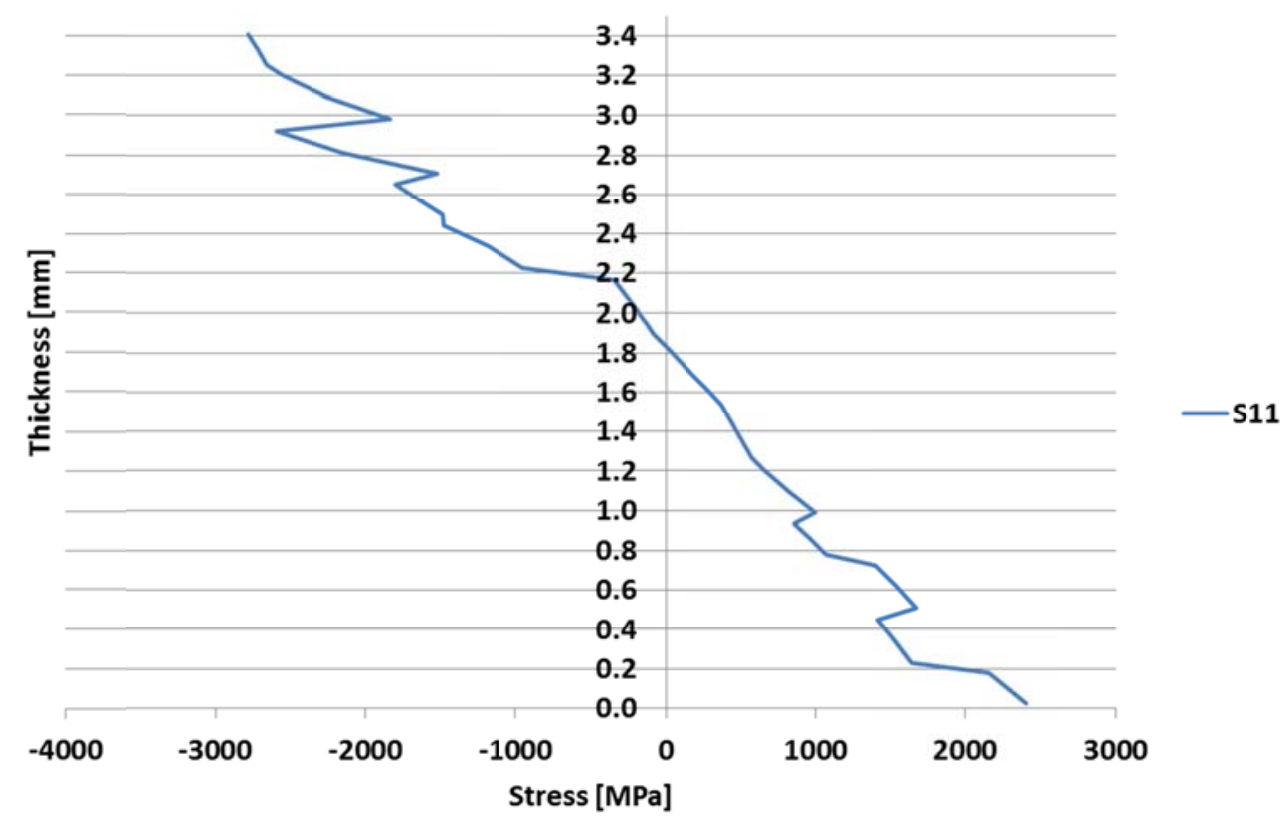

Figure 128: Type $\mathrm{C} \sigma_{11}$ Stress (S11) at the moment of layer 14 fiber breakage

Figure 129 shows the $\sigma_{22}$ for the FE most critical element at fiber failure onset. For this lay-up, layers from $1.4 \mathrm{~mm}$ to $3.4 \mathrm{~mm}$ had $\sigma_{22}$ considerably higher than the layer closer to the cylinder internal surface.

The intensity of $\sigma_{22}$ was smaller in external layers and the highest value happened at 2.85 $\mathrm{mm}$. 


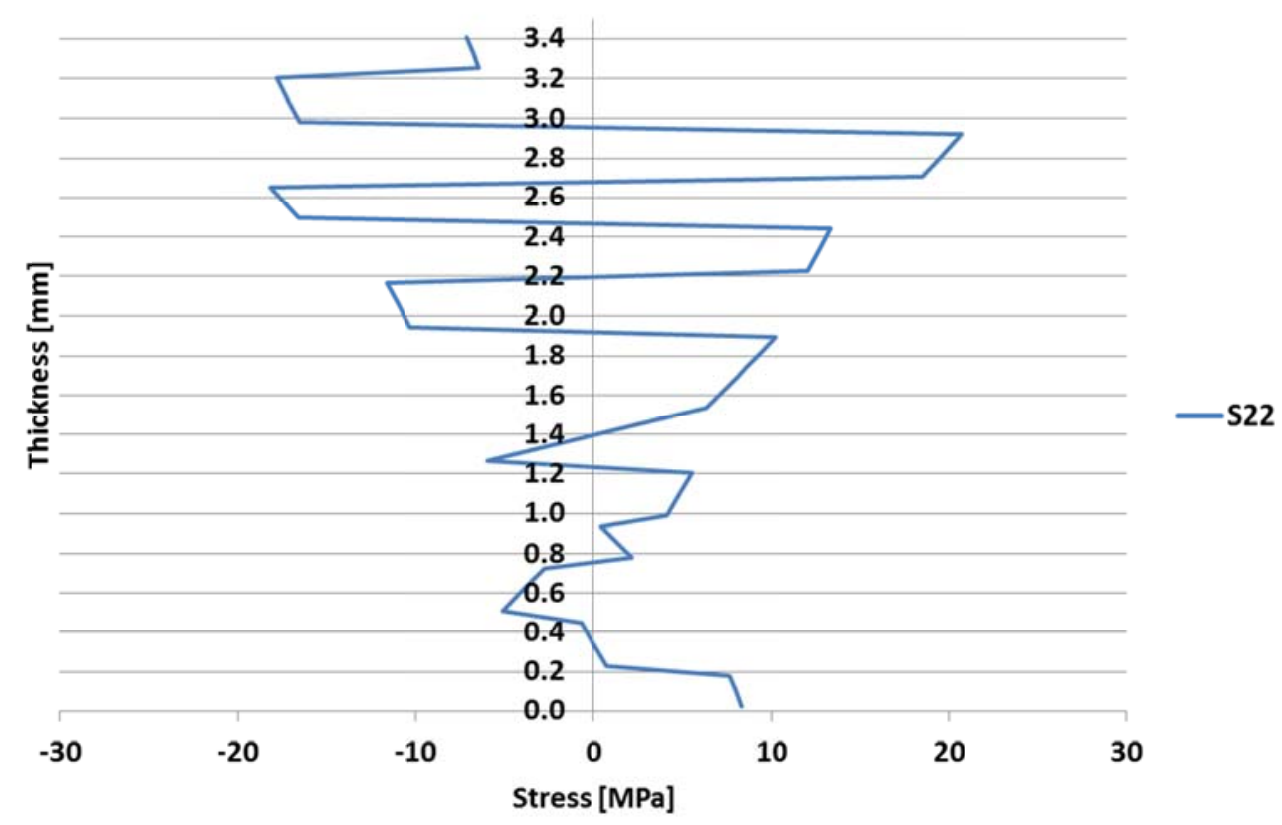

Figure 129: Type $\mathrm{C} \sigma_{22}$ Stress (S22) at the moment of layer 14 fiber breakage

Figure 130 shows the $\tau_{12}$ for the FE most critical element at fiber failure onset. For the shear stress, the external layers had considerably high shear stress, but they were not the most loaded ones. The highest shear stress occured at $3.2 \mathrm{~mm}$ (absolute value). 


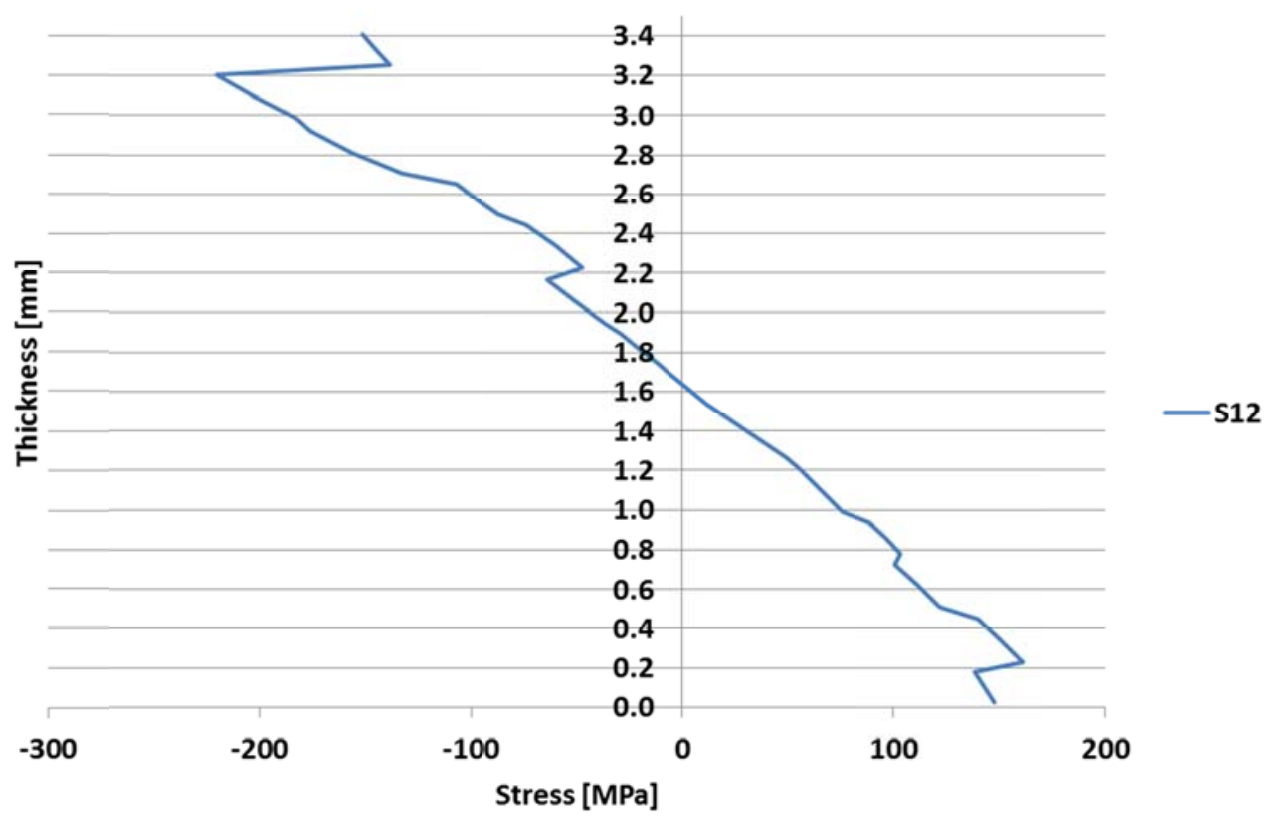

Figure 130 Type $\mathrm{C} \tau_{12}$ Stress (S12) at the moment of layer 14 fiber breakage

From $0.2 \mathrm{~mm}$ to $3.2 \mathrm{~mm}$, the shear stress showed a similar behavior to $\sigma_{11}$, i.e. $\tau_{12}$ decreased, than it became null at $1.6 \mathrm{~mm}$ and, yet decreased until $3.2 \mathrm{~mm}$. It is important to notice that for lamina coordinate system, the sign of shear stress is not important, once the effect on lamina of a positive shear stress is the same of a negative shear stress.

As for the previous cylinders analysis, the following figures improve the comprehension of the simulation results for damage calculation. These figures show the damage effect on each layer. As mentioned before, the impactor head as well as the composite lay-up were also shown to assist the comprehension of the impact damage. These figures show the final damage calculation results. 


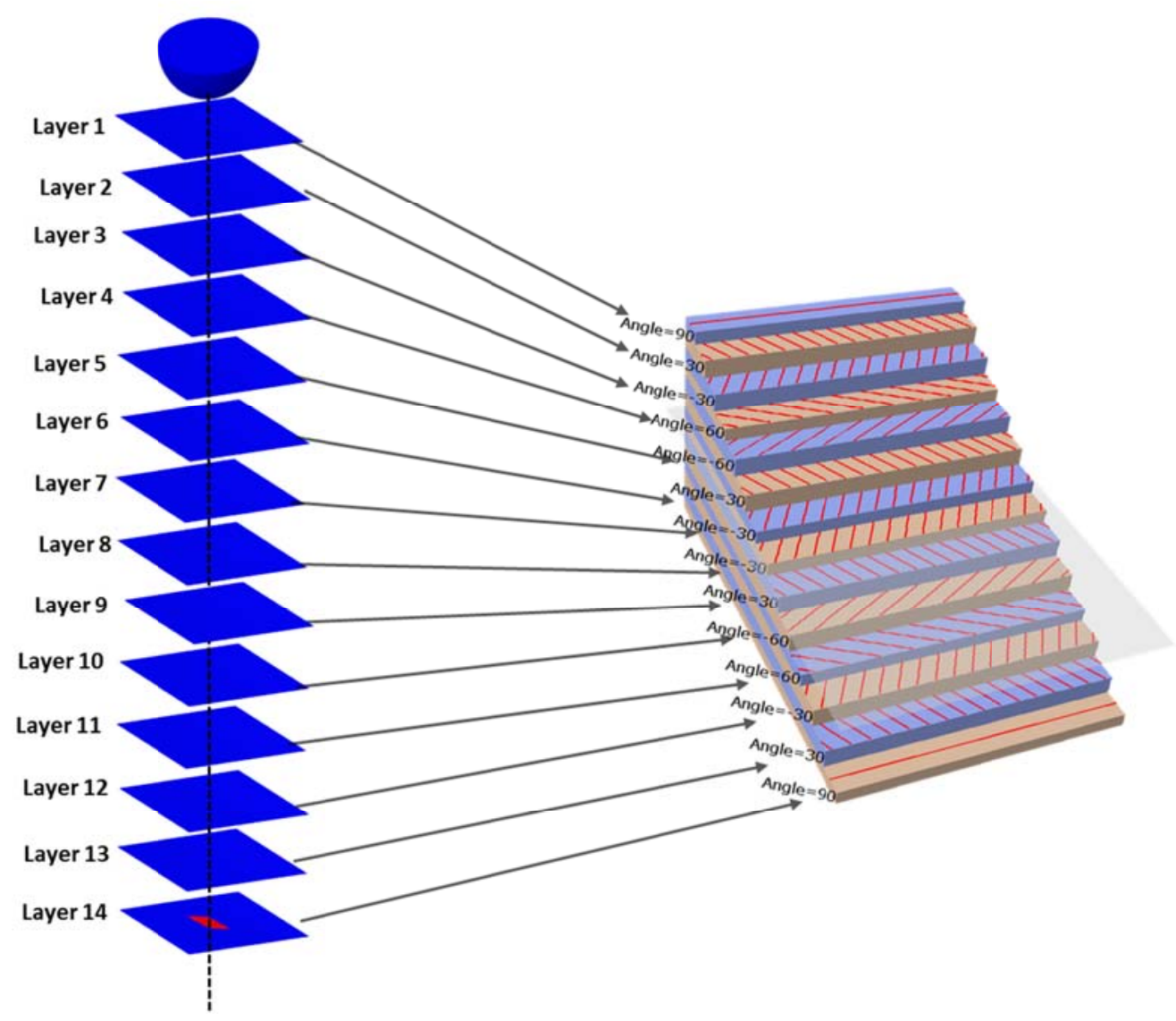

Figure 131: Type $C: d_{1}$ damage through the thickness

Figure 131 shows the layer- by- layer results for $d_{1}$. Once again, only layer 14 has been damaged. It is important to mention that $\mathbf{d}_{1}$ has not been calculated for compressive $\sigma_{11}$, despite $\mathrm{E}_{11}$ decreasing as established by the EQ.(15).

For a better understanding of matrix damage, Figure 132 and Figure 133 must be analyzed together, as the damage variables $\mathrm{d}_{2}$ and $\mathrm{d}_{6}$ are related to matrix damage process. 


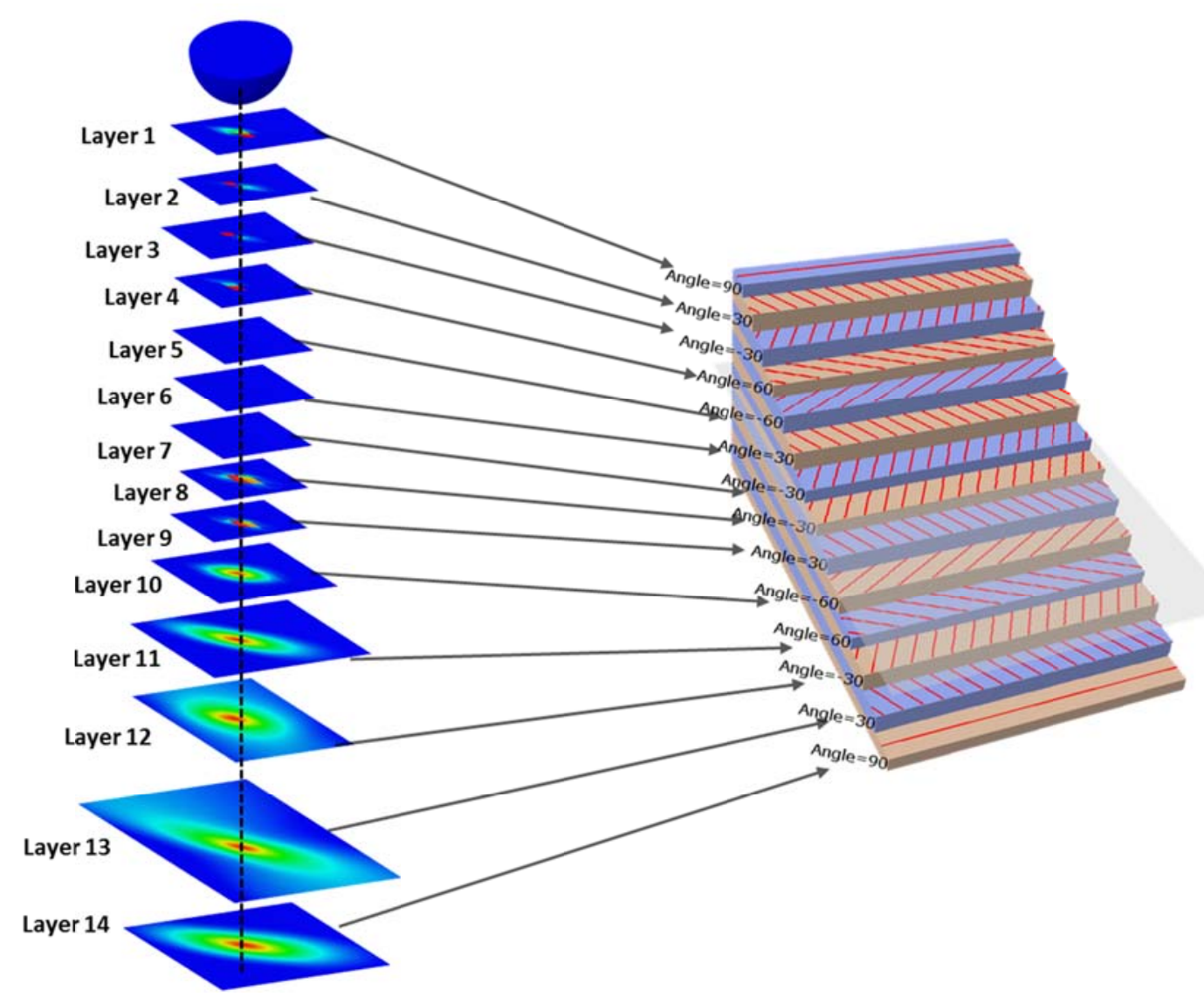

Figure 132: Type $C: d_{2}$ damage through the thickness

From those figures, it is noticed that layer 1 had some damage, but it was not the most damaged layer. For both damage variables, the damage intensity as well as the damage area decreased from layer 1 to layer 5 . In layer 5 , there is no matrix damage due to $d_{2}$, but this layer has seemed to be damaged due to $d_{6}$. Layers 6 and 7 also have not seemed to be damaged due to $\mathrm{d}_{2}$. However layer 6 had still some damage due to $\mathrm{d}_{6}$ while layer 7 has not presented any kind of damage.

In layer $8, \mathrm{~d}_{2}$ was calculated again, but there was no $\mathrm{d}_{6}$ in this lamina. The damage variables have been detected for all the rest of layers; the damage intensity as well as the affected area also increased from layer 9.

Figure 132 shows that, for $\mathrm{d}_{2}$, the highest affected areas were in layers 13 and 14 , as well as their highest intensity. For $\mathrm{d}_{6}$, the highest affected areas were in layers 12 and 13 (Figure $133)$. 


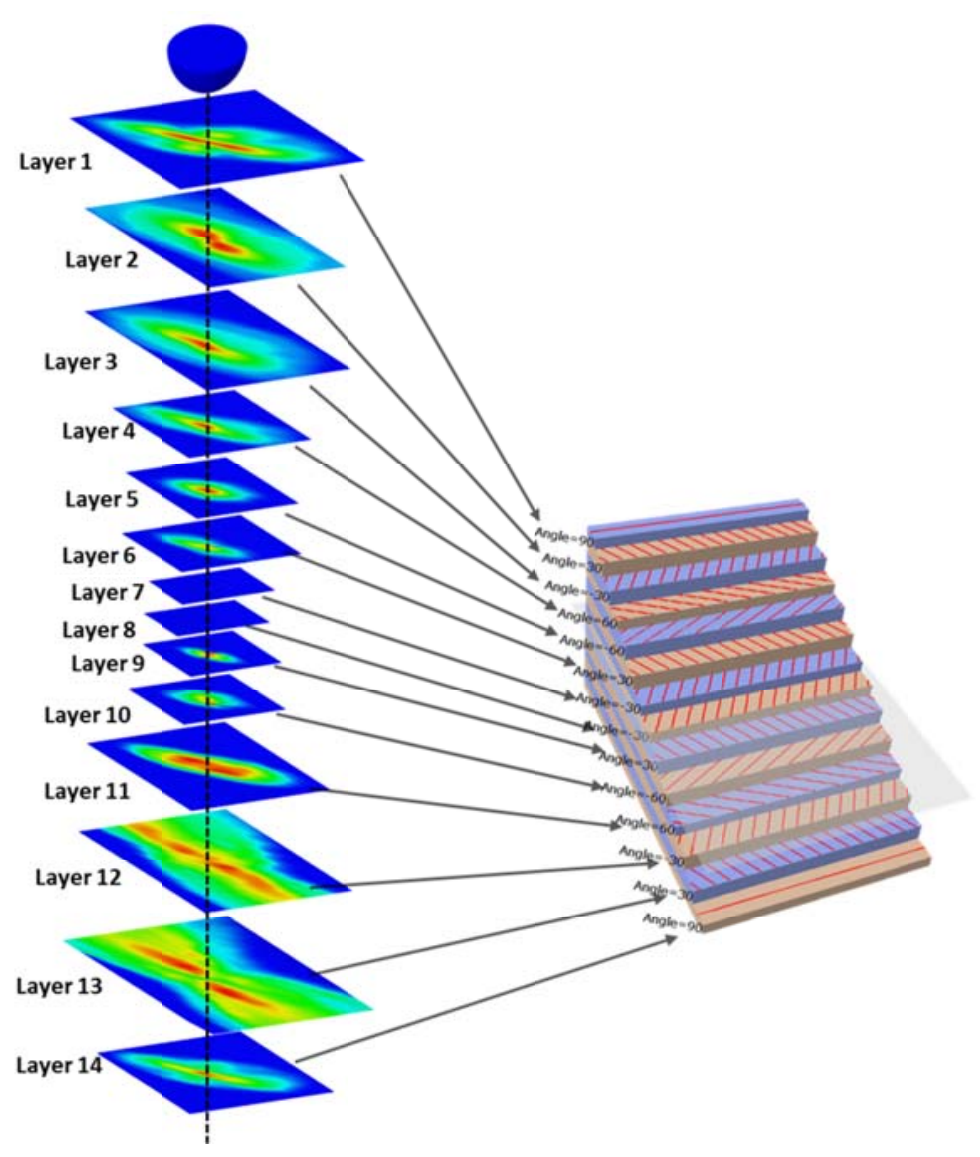

Figure 133: Type $C: d_{6}$ damage through the thickness.

Figure 134 shows the extension of $d_{6}$ in layer 12 . This is the highest cylinder surface area damaged. It is observed that $\mathrm{d}_{6}$ spread for a considerable part of the cylinder surface.

The most damaged area was closer to the impact area, as expected, but the bottom of the cylinder also showed a considerably damaged area and, its intensity is relatively high. On the other hand, the cylinder laterals (Figure 134) are also damaged, but the intensities are rather low. 


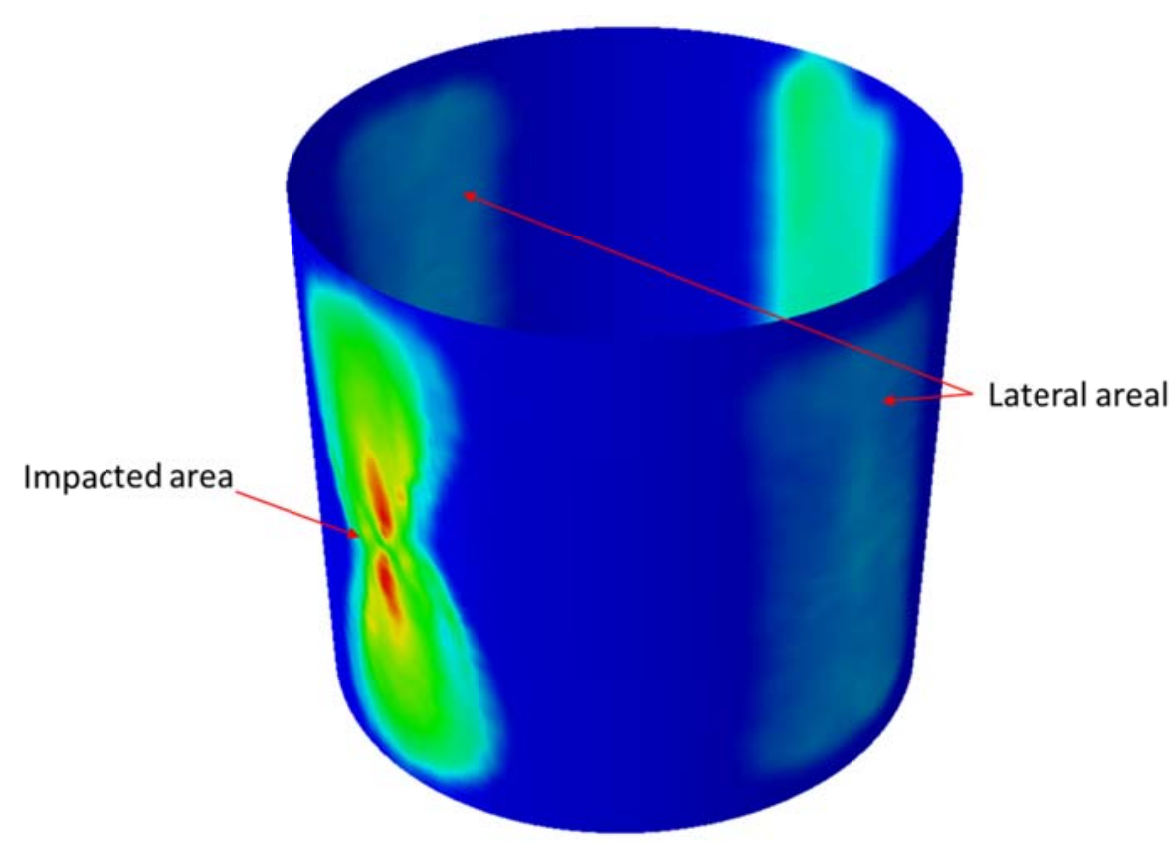

Figure 134: Shear damage $\left(\mathrm{d}_{6}\right)$ at layer 12.

Figure 135 shows the damage variables through the thickness for the FE most critical element at the end of simulation. As already mentioned, the fiber damage only happened in the cylinder internal layer. For matrix damage $\left(\mathrm{d}_{2}\right.$ and $\left.\mathrm{d}_{6}\right)$, on one hand, the layer at $0.0 \mathrm{~mm}$ (internal cylinder layer) has been the most damaged layer. However, on the other hand, the external cylinder layer at $3.5 \mathrm{~mm}$ (layer 1 ) has been not very much damaged. In fact, layers from $2.2 \mathrm{~mm}$ to $3.28 \mathrm{~mm}$ have been more damaged.

Also, the layer 7 (from $1.6 \mathrm{~mm}$ to $1.8 \mathrm{~mm}$ ) has been completely undamaged. Once more, the pine tree pattern has not been identified. 


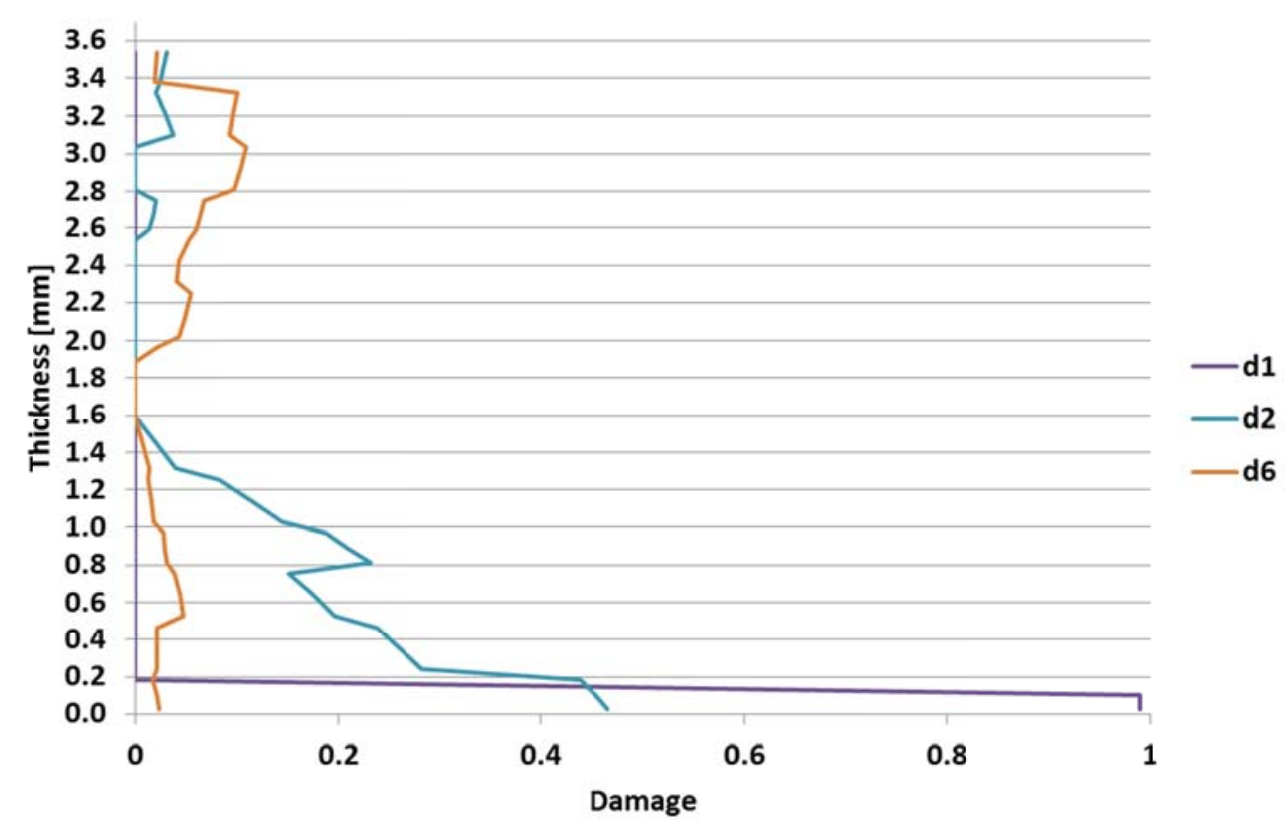

Figure 135: Type C damage variables through thickness.

\subsubsection{IMPACT SIMULATION CONCLUSIONS}

Impact was a very complex event. Several different factors may influence the structural behavior such as, material type, energy level, damage mechanism, structure geometry, etc.

This work showed that the cylindrical geometry adds complexity and, it has a huge influence on the structural behavior.

Several simulations have been performed to find "the best" FE model configuration for simulating the impact on investigated cylinders. Those simulations showed the huge influence on the results due to contact algorithm and damping. Moreover, the computational simulations showed how the damping affects the damage calculation, i.e. increasing the damping and decreasing the damage intensity.

Regarding the cylinders lay-up, type A was the most damaged cylinder, its damage intensity was higher than that shown by type B and C. However, this damage was much more concentrated. None of the type A damage variables spread on the cylinder surface as shown by $\mathrm{d}_{6}$ for cylinders type $\mathrm{B}$ and $\mathrm{C}$.

For all cylinders type, the classical pine tree shape for damage has not been identified, regarding the simulation results. However, it has not been possible ensure that it really happened for the coupons, once it has not been possible to perform the other tests. 
Finally, a detailed investigation of damage for each ply must be performed on the cylinders to verify the model accuracy to predict damage and its extension. Also, a delamination criterion must be implemented in the material model. Despite the lack of a delamination criterion, the intra-ply model has predicted both the damage and the type of damage in the investigated cylinder structures very well. 


\section{Final Conclusions ANd Future Perspectives}

Regarding the mathematical formulation of the new material model based on Continuum Damage Mechanics (CDM), it is possible to conclude that the FE model has shown a good performance, because it predicted the lamina failure as well as simulated the progressive failure of long fibers unidirectional composite laminates with certain accuracy using not such long computational time. Moreover, the material model developed in this work represents a new approach to detect the damage onset on composite lamina and its evolution accounting the influence of ply orientation. This can be confirmed by the comparisons among both numerical and experimental results.

Regarding the implementation of the material model proposal, it is concluded that FORTRAN subroutines linked to the commercial finite element program ABAQUS ${ }^{\mathrm{TM}}$ have been implemented satisfactorily, despite some software limitations. Thus, user material subroutines defined as UMAT (for quasi-static simulations), and as VUMAT (for explicit dynamic simulations) are adequate to be used in a new material model implementation and to simulate the composite structure behavior as well. The advantage of using those subroutines is that the FE model construction and analyses of the results are aided by the ABAQUS ${ }^{\mathrm{TM}}$ preprocessor (e.g. mesh algorithm; complex geometries), solver (e.g. contact algorithms) and post-processor tools (e.g. strain and stress gradient visualization in each layer).

Considering the set of experiments, it is feasible to conclude that they have been adequate to characterize the material elastic constants, to determine the strength limits, as well as to identify the damage model parameters and to evaluate the proposed material model potentialities and limitations. The UD carbon fibers coupons used in this work have beenmanufactured by using filament winding process. Due to manufacturing process, the coupons have shown some curvature, which produces some effects. These effects were a negligible for quasi-static tensile and four- point bending tests, but they have been considered for compression tests, once the coupon behaves as a beam-column decreasing the critical load for a perfect straight coupon. The impact tests had shown many important results, regarding the behavior of cylinders. Also, those tests represent a real challenge to be simulated by FE.

Considering the Finite Element Analyses in order to simulate the experiments, mainly based on the comparison between the numerical analyses and the experimental results, it has been possible to discuss the advantages and disadvantages of the investigated computational 
models, showing the influence of simulation parameters (e.g. analysis step size and mesh density). The material model was checked to simulate the failure behavior of unidirectional composite laminates under tension, compression and bending loadings. For this set of quasistatic tests, the material model has shown good results, even to predict the compression tests, where buckling occurs in almost all tests. Moreover, material model reproduced the beginning of the impact tests very well. However, the final of the numerical impact response need to be improved. In fact, the material model showed some limitations, mostly for the impact on cylinders, where the lack of delamination criteria as well as the absence of information about cylinders damping effects.

Based on the conclusions described above, it is possible to highlight some perspectives for future works by:

$\checkmark$ Studing damping phenomena of the composite cylinder, using dynamic experiments and other numerical models;

$\checkmark$ Formulating a new finite element, including delaminations and extended formulation. For example, it is possible to implement this new element via UEL (User Element). In other words, this subroutine in FORTRAN can be linked to the program ABAQUS ${ }^{\mathrm{TM}}$, and the FE model can be performed by using a new finite element;

$\checkmark$ Performing indentation tests on composite cylinders and comparing to the numerical analyses in order to evaluate the material model for this loading case;

$\checkmark$ Including delamination mode only in the material model, i.e. improving the UMAT in order to simulate the separation of plies observed during the impact tests. 


\section{Publications}

Here it is a list of publications developed by the author during the $\mathrm{PhD}$ work:

1. Ribeiro, Marcelo Leite, Tita, Volnei, Vandepitte, Dirk. A new damage model for composite laminates. Composite Structures, v.94, p.635 - 642, 2012.

2. Ribeiro, Marcelo L., Tita, Volnei. Bonded joints design aided by computational tool. Journal of Aerospace Technology and Management (Online). , v.4, p.275 - 288, 2012.

3. RIBEIRO, M. L., VANDEPITTE, D., TITA, V. Damage model and progressive failure analyses for filament wound laminates. Applied Composite Materials. , 2013

4. RIBEIRO, M. L., MARTINS, T. H. P., SARTORATO, M., FERREIRA, G. F. O., TITA, V., Vandepitte, Dirk. Experimental analysis of low energy impact in filament winding cylinders. International Journal of Vehicle Structures and Systems., v.4(4), p. 118-122, 2012.

5. MEDEIROS, R., RIBEIRO, M. L., FERREIRA, G. F. O., MARQUES, F. D., TITA, V. Numerical and experimental dynamic analyses in smart composites. International Journal of Vehicle Structures and Systems. , v.4(4), p.141-147, 2012.

6. SARTORATO, M., MEDEIROS, R., RIBEIRO, M. L., TITA, V. Transversal shear caracterisation of thick laminated composite using a representative volume element (rve). International Journal of Vehicle Structures and Systems., v.4(4), p. 136-140, 2012.

7. MEDEIROS, R., DANIEL, G. S., RIBEIRO, M. L., TITA, V. Ferramenta computacional para análise de fadiga de estruturas em compósito In: $1^{\circ}$ Encontro LusoBrasileiro de Engenharia de Compósitos, 2013, Porto. Proceedings of $1^{\mathrm{o}}$ Encontro Luso-Brasileiro de Engenharia de Compósitos. , 2013.

8. SARTORATO, M., TITA, V., MEDEIROS, R., RIBEIRO, M. L. A finite element for active composite plates with piezoelectric layers applied to composite cylinders In: CONEM, 2012, Natal. VII Congresso Nacional de Engenharia Mecânica., 2012.

9. RIBEIRO, M. L., MEDEIROS, R., SARTORATO, M., TITA, V. Damage detection in carbon fiber filament winding cylinders using smart piezoceramic materials In: VII Congresso Nacional de Engenharia Mecânica - CONEM2012, 2012, São Luiz. VII Congresso Nacional de Engenharia Mecânica. , 2012.

10. RIBEIRO, M. L., SARTORATO, M., FERREIRA, G. F. O., TITA, V., Vandepitte, Dirk. Evaluation of progressive failure of composite filament winding structure under complex loads In: FIRST BRAZILIAN CONFERENCE ON COMPOSITE MATERIALS, 2012, Natal. Proceedings of BCCM. C.A.Cimini Jr and J.D.D. Melo, 2012. v.1. 
11. RIBEIRO, M. L., SARTORATO, M., FERREIRA, G. F. O., TITA, V., Vandepitte, Dirk. Experimental analysis of low energy impact in filament winding cylindres In: FIRST BRAZILIAN CONFERENCE ON COMPOSITE MATERIALS, 2012, Natal. Proceedings of BCCM. C.A.Cimini Jr and J.D.D. Melo, 2012. v.1.

12. MEDEIROS, R., SARTORATO, M., RIBEIRO, M. L., Vandepitte, Dirk, TITA, V. Numerical and experimental analyses about SHM metrics using piezoelectric materials In: International Conference on Noise and Vibration Engineering, 2012, Leuven. Proceedings of ISMA 2012., 2012.

13. MEDEIROS, R., RIBEIRO, M. L., FERREIRA, G. F. O., MARQUES, F. D., TITA, V. Numerical and experimental dynamic analyses in smart composites In: FIRST BRAZILIAN CONFERENCE ON COMPOSITE MATERIALS, 2012, Natal. Proceedings of BCCM. C.A.Cimini Jr and J.D.D. Melo, 2012. v.1.

14. SIMOES, A., MEDEIROS, R., RIBEIRO, M. L., TITA, V., MARQUES, F. D. Piezofiber composite sensor tailoring using genetical algorithm In: 10th World Congress on Computational Mechanics, 2012, São Paulo. Proceedings of 10th WCCM., 2012.

15. RIBEIRO, M. L., MARTINS, T., Vandepitte, Dirk, TITA, V. Progressive failure analysis of low energy impact in carbon fiber filament winding cylinders In: 10th World Congress on Computational Mechanics (WCCM 2012), 2012, São Paulo. Proceedings of WCCM. , 2012.

16. SARTORATO, M., MEDEIROS, R., RIBEIRO, M. L., TITA, V. Transversal shear caracterisation of thick laminated composite using a representative volume element (rve) In: FIRST BRAZILIAN CONFERENCE ON COMPOSITE MATERIALS, 2012, Natal. Proceedings of BCCM. , 2012.

17. RIBEIRO, M. L., TITA, V. Failure models for composite structures under compression loading In: 21st INTERNATIONAL CONGRESS OF MECHANICAL ENGINEERING, 2011, Natal. 21st INTERNATIONAL CONGRESS OF MECHANICAL ENGINEERING. , 2011.

18. MEDEIROS, R., TITA, V., RIBEIRO, M. L., Venturini Neto, S. N. Study of aeronautic fastened hybrid joints metal-composite In: 21th International Congress of Mechanical Engineering (COBEM 2011), 2011, Natal 21th International Congress of Mechanical Engineering (COBEM 2011). , 2011.

19. RiBeiro, M. L., Barbosa, A. A. F., BORGES, E. N., BASILE, T. F., TITA, V. Design and analysis of aeronautical repair: fastened $\mathrm{x}$ bonded In: Congresso SAE Brasil, 2010, São Paulo. Congresso SAE Brasil. , 2010.

20. ANGeliCO, R. A., RIBEIRO, M. L., Silvestrini, R. H., Di Salvo, F. E., TITA, V. Investigação do comportamento mecânico de junções de compósitos termoplásticos In: Congresso Nacional de Engenharia Mecânica - CONEM, 2010, Campina Grande. Congresso Nacional de Engenharia Mecânica. , 2010. 
21. Angelico, R. A., RIBEIRO, M. L., Angelo, M. V., TITA, V.Composite failure analysis for an aircraft structure In: 20th International Congress of Mechanical Engineering (COBEM 2009), 2009, Gramado. Procedings of 20th International Congress of Mechanical Engineering. , 2009.

22. RIBEIRO, M. L., ANGELICO, R. A., TITA, V. Development of a computational tool for bonded joint analysis In: 11th Pan-American Congress of Applied Mechanics PACAM XI, 2009, Foz do Iguaçú. Proceedings of PACAM XI. , 2009.

23. RIBEIRO, M. L., ANGELICO, R. A., TITA, V. Investigation of failure analysis on single and double lap bonded joints In: Brazilian Symposium on Aerospace Eng. \& Applications, 2009, São José dos Campos. Proceedings of 2009 Brazilian Symposium on Aerospace Eng. \& Applications. , 2009.

24. ANGELICO, R. A., RIBEIRO, M. L., TITA, V. Investigation of parameters for composite progressive failure material model In: 11th Pan-American Congress of Applied Mechanics - PACAM XI, 2009, Foz do Iguaçú. Proceedings of PACAM XI. , 2009.

25. RIBEIRO, M. L., ANGELICO, R. A., TITA, V., DANIEL, G. S. Parameters effect study on the mechanical behavior of single and double lap bonded joints In: 20th International Congress of Mechanical Engineering (COBEM 2009), 2009, Gramado. Proceedings of 20th International Congress of Mechanical Engineering. , 2009.

26. TITA, V., ANGEliCO, R. A., RIBEIRO, M. L. Failure Mechanisms Modeling of Hybrid Joints (Metal-Composite) In: Brazil AFOSR Workshop on Advanced Structural Mechanics and Computational Mathematics, 2008, Campinas. Brazil AFOSR Workshop on Advanced Structural Mechanics and Computational Mathematics. , 2008. 


\section{REFERENCES}

Abrate, S. (1998). Impact on composite structures. Cambridge University Press.

Abrate, S. (2001). Modeling of impacts on composite structures. Composite Structures, 51(2), 129-138.

Allix, O., Ladevèze, P., \& Vittecoq, E. (1994). Modelling and identification of the mechanical behaviour of composite laminates in compression. Composites Science and Technology, 51(1), 35-42.

Anderson, T. (1995). Fracture Mechanics - Fundamentals and Applications (Second Edition ed.). (C. Press, Ed.)

ASTM D3039. (2006). Standard Test Method for Tensile Properties of Polymer Matrix Composite Materials.

ASTM D3410. (2003). Standard Test Method for Compressive Properties of Polymer Matrix Composite Materials with Unsupported Gage Section by Shear Loading.

ASTM D3518. (2007). Standard Test Method for In-Plane Shear Response of Polymer Matrix Composite Materials by Tensile Test of a $64.5^{\circ}$ Laminate.

ASTM D4255. (2007). Standard Test Method for In-Plane Shear Properties of Polymer Matrix Composite Materials by the Rail Shear Method. Standard Test Method for InPlane Shear Properties of Polymer Matrix Composite Materials by the Rail Shear Method.

ASTM D7139. (2007). Standard Test Method for Measuring the Damage Resistance of a Fiber-Reinforced Polymer Matrix Composite to a Drop-Weight Impact Event.

ASTM D7139. (2007). Standard Test Method for Measuring the Damage Resistance of a Fiber-Reinforced Polymer Matrix Composite to a Drop-Weight Impact Event. ASTM.

ASTM D790. (2008). Standard Test Methods for Flexural Properties of Unreinforced and Reinforced Plastics and Electrical Insulating Materials.

Ballère, L., Viot, P., Lataillade, J.-L., Guillaumat, L., \& Cloutet, S. (2009). Damage tolerance of impacted curved panels. International Journal of Impact Engineering, 36(2), 243253.

Belytschko, T., Liu, W. K., \& Moran, B. (2000). Nonlinear finite elements for continua and structures. Wiley. 
Budiansky, B., \& Fleck, N. (1993). Compressive failure of fibre composites. Journal of the Mechanics and Physics of Solids, 41(1), 183-211.

Callister, W. D. (2011). Fundamentals of Materials Science and Engineering. (W. Anderson, Ed.) John Wiley \& Sons, Inc.

Carvalho, O. d. (2006). Influência da configuração de bobinagem no comportamento mecânico de cilindros de compósito polimérico. Master's thesis, Instituto de Pesquisas Energéticas e Nucleares.

Christoforou, A. P., \& Yigit, A. S. (2009). Scaling of low-velocity impact response in composite structures. Composite Structures, 91(3), 358-365.

Daniel, I. M., \& Ishai, O. (2006). Engineering Mechanics of Composite Materials (2nd ed.). Oxford University Press.

Dassault Systèmes Simulia Corp. (2010). Abaqus 6.10. Providence, RI, USA.

Donadon, M. V., Frascino, S. M., Arbelo, M. A., \& Faria, A. R. (2009). A Three-Dimensional Ply Failure Model for Composite Structures. International Journal of Aerospace Engineering, 2009, 22.

Donadon, M., Iannucci, L., Falzon, B., Hodgkinson, J., \& Almeida, S. d. (2008). A progressive failure model for composite laminates subjected to low velocity impact damage. Computers \& Structures, 86(11-12), 1232-1252.

Flatscher, T., \& Pettermann, H. (2011). A constitutive model for fiber-reinforced polymer plies accounting for plasticity and brittle damage including softening - Implementation for implicit FEM. Composite Structures, 93(9), 2241-2249.

Hashin, Z. (1980). Failure Criteria for Unidirectional Fiber Composites. Journal of Applied Mechanics, 47(2), 329-334.

Herakovich, C. (1998). Mechanics of Fibrous Composites (first edition ed.). (J. W. I\&, Ed.)

Hild, F., \& Roux, S. (2008). CorreliQ4: A software for "finite element" displacement fiels measurements by digital image correlation. Paris: Université Paris/LMT-Cachan.

Hong, Y., He, X., \& Wang, R. (2012). Vibration and damping analysis of a composite blade. Materials \&amp; Design, 34(0), 98-105.

Kachanov, L. M. (1986). Introduction to Continuum Damage Mechanics. Dordrecht: Martinus Nijhoff Publishers.

Khalili, S., Soroush, M., Davar, A., \& Rahmani, O. (apr. de 2011). Finite element modeling of low-velocity impact on laminated composite plates and cylindrical shells. Composite Structures, 93(5), 1363-1375. 
Kobayashi, S., \& Kawahara, M. (2012). Effects of stacking thickness on the damage behavior in CFRP composite cylinders subjected to out-of-plane loading. Composites Part A: Applied Science and Manufacturing, 43(1), 231-237.

Kyriazoglou, C., \& Guild, F. (2007). Finite element prediction of damping of composite GFRP and CFRP laminates a hybrid formulation vibration damping experiments and Rayleigh damping. Composites Science and Technology, 67, 2643-2654.

Ladeveze, P., \& LeDantec, E. (1992). Damage modelling of the elementary ply for laminated composites. Composites Science and Technology, 43(3), 257-267.

Lapczyk, I., \& Hurtado, J. A. (2007). Progressive damage modeling in fiber-reinforced materials. Composites Part A: Applied Science and Manufacturing, 38(11), 23332341.

Laval, C. (2006). CADWIND 2006 - 20 years of filament winding experience. Reinforced Plastics, 50(2), 34-37.

Lemaitre, J. (1996). A course on damage mechanics (2nd ed.). Munich: Springer.

Lemaitre, J., \& Desmorat, R. (2005). Engineering Damage Mechanics - Ductile, Creep, Fatigue and Brittle Failures. Springer.

Lemaitre, J., \& Desmorat, R. (2005). Engineering Damage Mechanics: Ductile, Creep, Fatigue and Brittle Failures (First edition ed.). Springer.

Li, B. W., \& Goldberg, R. (2011). Finite-Element Model for Failure Study of TwoDimensional Triaxially Braided Composite. Journal of Aerospace Engineering, 34, J. Aerosp. Eng.

Lubineau, G. (2010). A Pyramidal Modeling Scheme for Laminates - Identification of Transverse Cracking. International Journal of Damage Mechanics, 19(4), 499-518.

Lubineau, G., \& Ladevèze, P. (2008). Construction of a micromechanics-based intralaminar mesomodel, and illustrations in ABAQUS/Standard. Computational Materials Science, 43(1), 137-145.

Maimí, P., Camanho, P., Mayugo, J., \& Dávila, C. (2007). A continuum damage model for composite laminates: Part I - Constitutive model. Mechanics of Materials, 39(10), 897-908.

Mangalari, P. (1999). Composite materials for aerospace app. Bull. Mater. Sci., 22, 657-664.

Matzenmiller, A., Lubliner, J., \& Taylor, R. L. (1995). A constitutive model for anisotropic damage in fiber-composites. Mechanics of Materials, 20(2), 125-152.

Meer, F. V., \& Sluys, L. (2009). Continuum models for the analysis of progressive failure in composite laminates. Journal of Composite Materials, 43, 31-56. 
Menna, C., Asprone, D., Caprino, G., Lopresto, V., \& Prota, A. (2011). Numerical simulation of impact tests on GFRP composite laminates. International Journal of Impact Engineering, 38(8â€“"9), 677-685.

Minak, G., Abrate, S., Ghelli, D., Panciroli, R., \& Zucchelli, A. (2010). Residual torsional strength after impact of CFRP tubes. Composites Part B: Engineering, 41(8), 637-645.

Morozov, E. (2006). The effect of filament-winding mosaic patterns on the strength of thinwalled composite shells. Composite Structures, 76(1-2), 123-129.

Pavan, R., Oliveira, B., Maghous, S., \& Creus, G. (2010). A model for anisotropic viscoelastic damage in composites. Composite Structures, 92(5), 1223-1228.

Pierron, F., \& Vautrin, A. (1996). The $10^{\circ}$ off-axis tensile test: A critical approach. Composites Science and Technology, 56(4), 483-488.

Pinho, S. T. (2005). Modelling failure of laminated composites using physically-based failure models. Ph.D. dissertation, Imperial College.

Proença, S. P. (2007). Análise não-linear de estruturas - Notas de Aula. EESC.

Puck, A., \& Schürmann, H. (1998). Failure analysis of FRP laminates by means of physically based phenomenological models. Composites Science and Technology, 58(7), 10451067.

Puck, A., \& Schürmann, H. (2002). Failure analysis of FRP laminates by means of physically based phenomenological models. Composites Science and Technology, 62(12-13), 1633-1662.

Quaresimin, M., Ricotta, M., Martello, L., \& Mian, S. (2013). Energy absorption in composite laminates under impact loading. Composites Part B: Engineering, 44(1), 133-140.

Reid, S. R., \& Zhou, G. (2000). Impact behaviour of fibre-reinforced composite materials and structures (1 edition ed.). CRC Press.

Ribeiro, M. L., Tita, V., \& Vandepitte, D. (2012). A new damage model for composite laminates. Composite Structures, 94(2), 635-642.

Schoeppner, G., \& Abrate, S. (2000). Delamination threshold loads for low velocity impact on composite laminates. Composites Part A: Applied Science and Manufacturing, 31(9), 903-915.

Schuecker, C., \& Pettermann, H. (2006). A continuum damage model for fiber reinforced laminates based on ply failure mechanisms. Composite Structures, 76(1-2), 162-173.

Simulia. (2010). ABAQUS 6.10 User Manual.

Skinner, M. L. (2006). Trends, advances and innovations in filament winding. Reinforced Plastics, 50(2), 28-33. 
Sun, J., Lee, K., \& Lee, H. (2000). Comparison of implicit and explicit finite element methods for dynamic problems. Journal of Materials Processing Technology, 105, 110-118.

Tay, T., Liu, G., Tan, S. V., Sun, C. X., \& Phan, D. (2008). Progressive Failure Analysis of Composites. Journal of Composite Materials, 42, 1921-1966.

Tita, V. (2003). Contribuição ao estudo de danos e falhas progressivas em estruturas de material compósito polimérico. Ph.D. dissertation, Escola de Engenharia de São Carlos.

Tita, V., Carvalho, J. d., \& Vandepitte, D. (2008). Failure analysis of low velocity impact on thin composite laminates: Experimental and numerical approaches. Composite Structures, 83(4), 413-428.

Tita, V., Carvalho, J., \& Vandepitte, D. (2008). Failure analysis of low velocity impact on thin composite laminates: experimental and numerical approaches. Journal of Composite Structures, 83, 413 - 428.

Travessa, A. T. (2006). Simulation of delamination in composites under quasi-static and fatigue loading using cohesive zone models. Ph.D. dissertation, Universitat de Girona.

Williams, K. V., Vaziri, R., \& Poursartip, A. (2003). A physically based continuum damage mechanics model for thin laminated composite structures. International Journal of Solids and Structures, 40(9), 2267-2300.

Wisnom, M. (1990). The effect of fibre misalignment on the compressive strength of unidirectional carbon fibre/epoxy. Composites, 21(5), 403-407.

Xiao, X. (2007). Evaluation of a composite damage constitutive model for PP composites. Composite Structures, 79(2), 163-173.

Yokozeki, T., Ogasawara, T., \& Ishikawa, T. (2005). Effects of fiber nonlinear properties on the compressive strength prediction of unidirectional carbon-fiber composites. Composites Science and Technology, 65(14), 2140-2147.

Yokozeki, T., Ogasawara, T., \& Ishikawa, T. (2006). Nonlinear behavior and compressive strength of unidirectional and multidirectional carbon fiber composite laminates. Composites Part A: Applied Science and Manufacturing, 37(11), 2069-2079.

Zabaras, N., \& Pervez, T. (1990). Viscous damping approximation of laminated anisotropic composite plates using the finite element method. Computer Methods in Applied Mechanics and Engineering, 81(3), 291-316.

Zhou, W. X., \& Gao, X. (2012). Damage and Failure of a Laminated Carbon Fiber Reinforced Composite under Low Velocity Impact. Journal of Aerospace Engineering. 
Zienkiewicz, O. C., \& Taylor, R. L. (2000). The Finite Element Method. Barcelona: Butterworth Heinemann.

Zukas, J. A., Nicholas, T., Swift, H. F., Greszczuk, L. B., \& Curran, D. R. (1982). Impact Dynamics. John Wiley \& Sons.

Zukas, J. A., Nicholas, T., Swift, H. F., Greszczuk, L. B., \& Curran, D. R. (1982). Impact Dynamics. John Wiley \& Sons. 
SUBROUTINE UMAT(STRESS, STATEV, DDSDDE, SSE, SPD, SCD,

1 RPL, DDSDDT, DRPLDE, DRPLDT,

STRAN, DSTRAN, TIME, DTIME, TEMP, DTEMP, PREDEF, DPRED, CMNAME,

3 NDI, NSHR, NTENS, NSTATV, PROPS, NPROPS, COORDS, DROT, PNEWDT,

C

CELENT, DFGRD@ , DFGRD1, NOEL, NPT, LAYER, KSPT, KSTEP, KINC)

C

INCLUDE 'ABA_PARAM. INC'

\section{CHARACTER $* 80$ CMNAME}

DIMENSION STRESS(NTENS), STATEV(NSTATV),

1 DDSDDE(NTENS, NTENS), DDSDDT (NTENS), DRPLDE (NTENS),

$2 \operatorname{STRAN}(N T E N S), \operatorname{DSTRAN}(\operatorname{NTENS}), \operatorname{TIME}(2), \operatorname{PREDEF}(1), \operatorname{DPRED}(1)$,

3 PROPS(NPROPS), $\operatorname{COORDS}(3), \operatorname{DROT}(3,3), \operatorname{DFGRDO}(3,3), \operatorname{DFGRD1}(3,3), \operatorname{PS}(3)$,

$\operatorname{AN}(3,3)$

double precision E11, E22, nu12, G12, nu21, Xt, Xc, d1, TF2, d2,

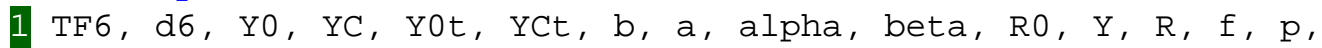

2 point_a, point_b, Y2C, starin1, teta, PS, AN, S12, E11C

parameter $(X t=X X, X C=X X, Y \Theta=\odot .09, Y C=2.07$,

$1 \mathrm{Y} \odot \mathrm{t}=0.092, \mathrm{YCt}=29.2, \mathrm{R} \Theta=15 . \odot$, epsilon $\Theta=0.003, \mathrm{~S} 12=\mathrm{XX}$,

$2 \mathrm{Y} 2 \mathrm{~T}=0.3)$

parameter $(b=1.02$, alpha $=1.0$, beta=3.๑, $n p=10, a=0.5)$

dimension orientation( $\mathrm{np}$ )

c Variables for read input file in order to assemble the orientation

C layer matrix

integer point, nc, ply

real(8) ori, thick, gamma1, gamma2, gamma3, gamma, dfdt1, dfdt2,

1 dfdt3, ep1a, ep1b, dep1, DP, ef, ep2_p, ep12_p

c Vari veis para energia

double precision Ed, Ed_rup

C parameter (Ed_rup=2.5)

C Variables for matrix damage under compression

double precision $m \_f, m_{-} y$, su_y, su_f, S12_f, S12_y, K_y

C

parameter $\left(m_{-} f=70.0, m_{-} y=62.5, \quad S 12 \_f=80.0, \quad S 12 \_y=47.0\right)$

parameter ( $\mathrm{m}_{-} \mathrm{f}=\mathrm{XX}, \mathrm{S} 12 \_\mathrm{f}=\mathrm{XX}$,

$1 \mathrm{~S} 12 \_\mathrm{y}=\mathrm{XX}$ )

C $\operatorname{parameter}($ job $=$ '3BP०90.inp', part $=$ 'composite')

C

C

PLY ORIENTATION

orientation $(1)=90 . \odot$

orientation $(2)=90 . \odot$

orientation $(3)=90 . \odot$

orientation $(4)=90.0$

orientation $(5)=90.0$

orientation $(6)=90.0$

orientation $(7)=90.0$

orientation $(8)=90.0$

orientation $(9)=90 . \odot$ 
orientation $(10)=90 . \odot$

$$
\text { teta=abs (orientation(layer)) }
$$

c teta $=30.0$ !orientation(LAYER)

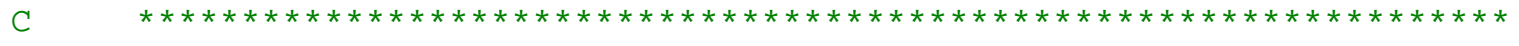

C

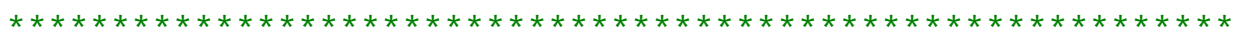

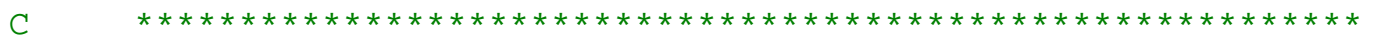

$\mathrm{E} 11=\operatorname{props}(1)$

$\mathrm{E} 22=\operatorname{props}(2)$

nu12 $=\operatorname{props}(3)$

$\mathrm{G} 12=\operatorname{props}(4)$

nu21 $=(\operatorname{props}(2) / \operatorname{props}(1)) * \operatorname{props}(3)$

C Inicialization of variables

$\mathrm{d} 1=0.0$

$\mathrm{d} 2=0.0$

d6 $=0.0$

ep12_p $=0.0$

ep2_p $=0.0$

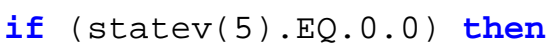
$p=0.0$

else if (statev(5).ne.๑.๑) then $p=\operatorname{statev}(5)$

end if

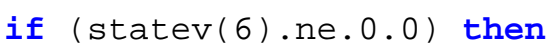

$\mathrm{d} 1$ = statev $(1)$

$\mathrm{d} 2=\operatorname{statev}(2)$

$\mathrm{d} 6=\operatorname{statev}(3)$

$\mathrm{E} 11=\operatorname{statev}(11)$

$\mathrm{E} 22$ = $\operatorname{statev}(12)$

end if

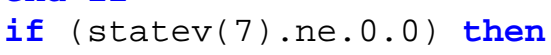

ep12_p=statev (8)

ep2_p=statev (9)

end if

if (E11.1t. $0.03^{*}$ props(1)) then

$\mathrm{E} 11=0.03^{*} \operatorname{props}(1)$

end if

$\mathrm{K} \_\mathrm{y}=\operatorname{statev}(13)$

C $\quad * * * * * * * * * * * * * * * * * * * * * * * * * * * * * * * * * * * * * * * * * * * * * * * * * * * * * * * * * * *$

C CONSTITUTIVE LAW

C $\quad * * * * * * * * * * * * * * * * * * * * * * * * * * * * * * * * * * * * * * * * * * * * * * * * * * * * * * * * * * *$

do $10 i=1,3$

do $20 j=1,3$

$$
\operatorname{ddsdde}(i, j)=\odot . \odot \text {; }
$$

ddsdde $(1,1)=(E 11 *(1.0-d 1)) /(1.0-($ nu12*nu21 $) *(1.0-d 2) *(1.0-d 1))$ 


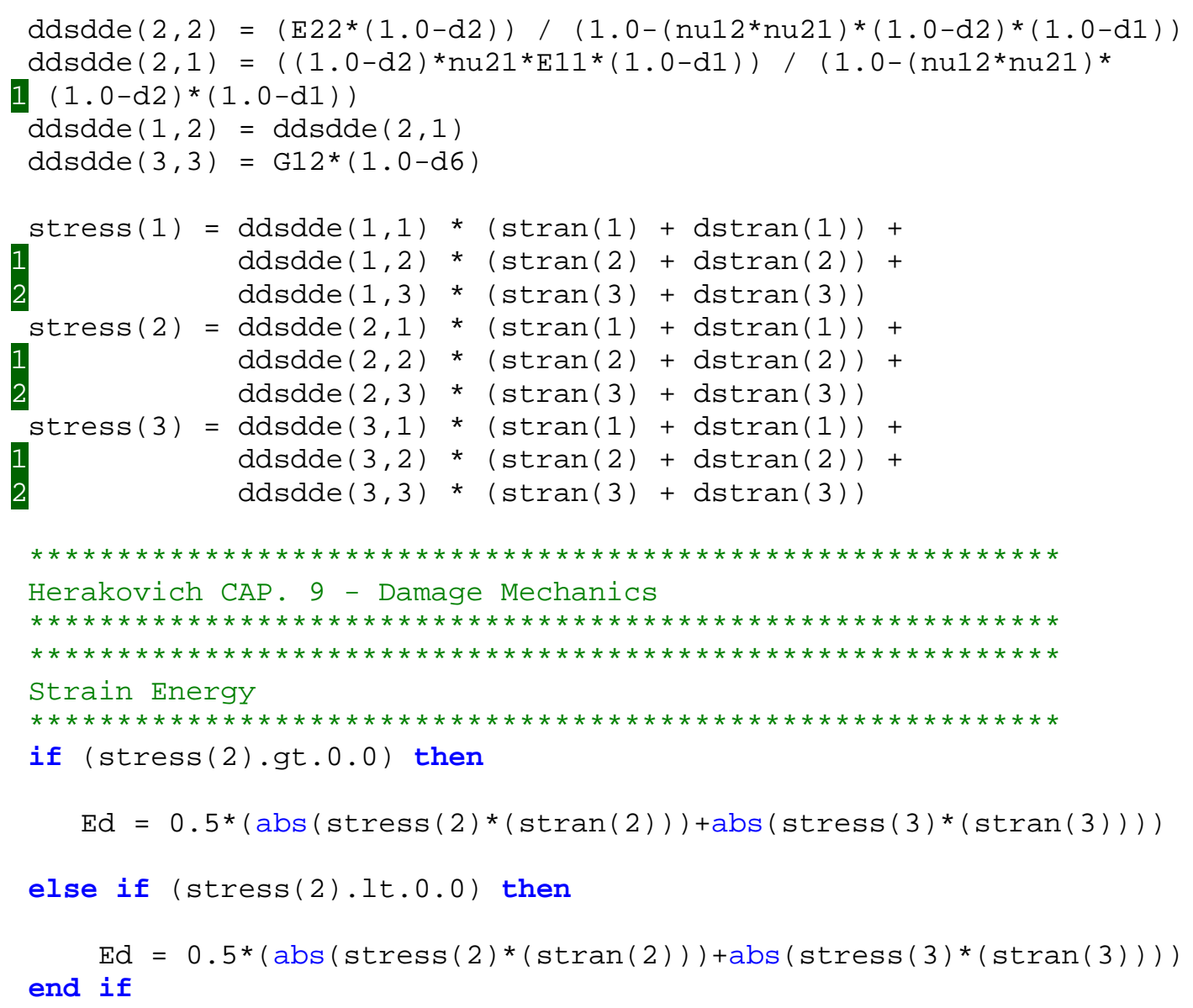

C

Fiber failure - Max tensile or Max Compression

if (stress(1).gt. $\odot . \odot)$ then

$$
\begin{aligned}
& f f=((\operatorname{stress}(1) / X t) * * 2 . \odot) \\
& \text { if }(f f . g e .1 . \odot) \text { then } \\
& d 1=0.99
\end{aligned}
$$

C

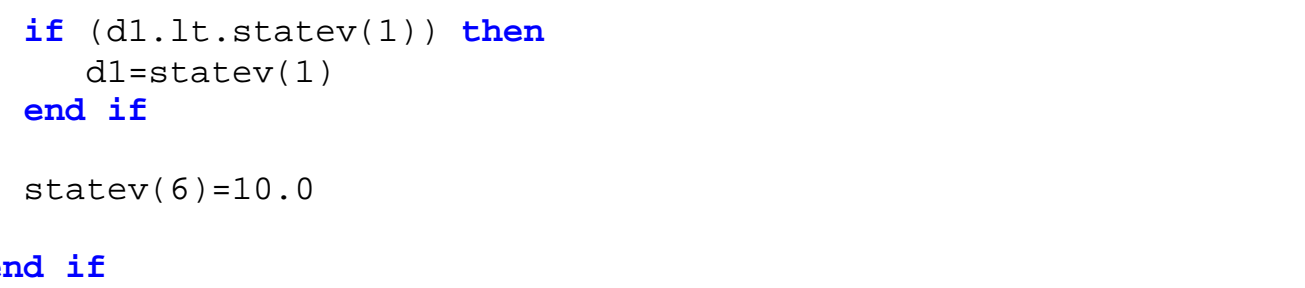

else if ( $\operatorname{stress}(1) \cdot 1 \mathrm{t} .0 .0)$ then

$\mathrm{ff}=(\operatorname{stress}(1) / \mathrm{Xc}) * * 2.0$

if (ff.ge.1.0) then

C

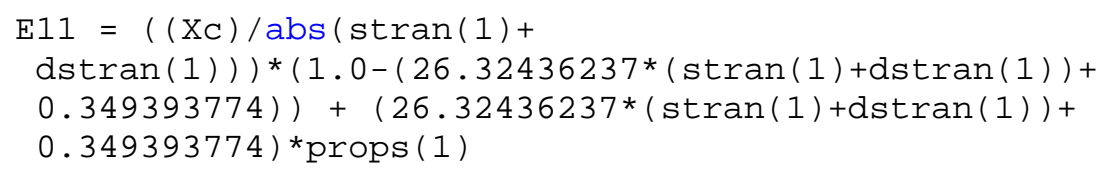


C

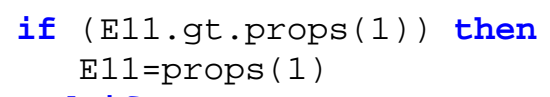

end if

statev $(6)=10.0$

end if

end if

C Matrix damage

C Failure criteria

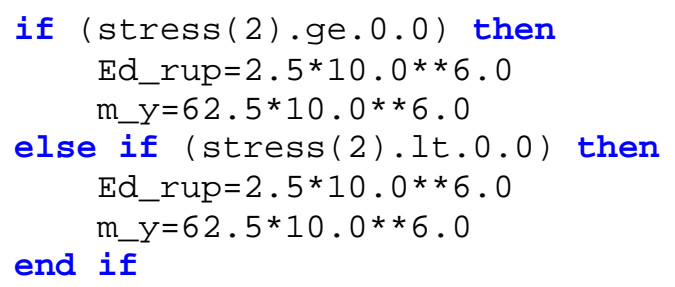

su_y $=\operatorname{sqrt}(((\operatorname{stress}(2) * * 2.0)+(\operatorname{stress}(3) * * 2.0)))-$

$1\left(-\mathrm{S} 12 \_\mathrm{y}+\left(\left(2 * \mathrm{~S} 12 \_\mathrm{y}\right) /\left(1+\left(\left(\operatorname{abs}(\operatorname{stress}(2)) / \mathrm{m}_{-} \mathrm{y}\right) * * 3.0\right)\right)\right)\right)$

C Verification of failure criteria and evolution of plastic surface

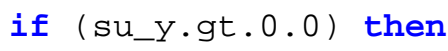

C Thermodynamic forces

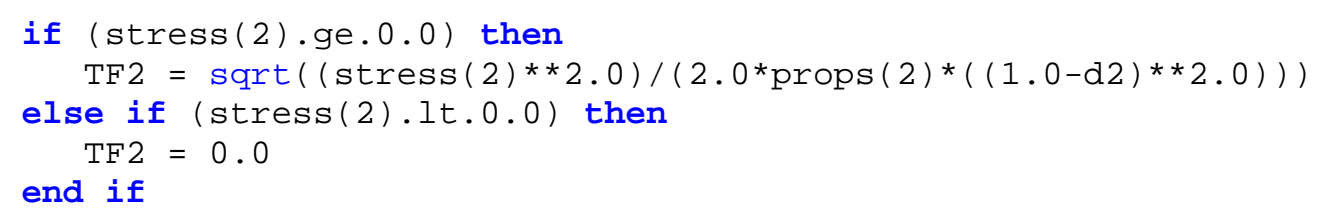

C

end if 


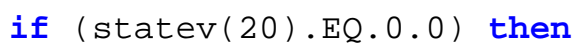

statev $(20)=(\odot .0019 *$ teta $+\odot .363) * \operatorname{TF} 6$

end if

if $\left(\left(s_{-} y \cdot g t \cdot \odot \cdot \odot\right)\right.$.and. (stress $\left.\left.(2) \cdot g t \cdot \odot \cdot \odot\right)\right)$ then

$d 2=\left(-0.000215^{\star}\right.$ teta +0.18752$){ }^{*} T F 2-\operatorname{statev}(19)$

if ((NPT.EQ.1).and.(KSPT.EQ.1).and.(layer.EQ.1)) then

C

end if

if (d2.lt.statev(2)) then

$\mathrm{d} 2=\operatorname{statev}(2)$

end if

if ((d2.ge.0.99).or.(Ed.ge.Ed_rup).or.

1

(stress(2).gt.65)) then $\mathrm{d} 2=\odot .99$ $d 6=0.99$

end if

end if

statev $(6)=10.0$

$d 6=(\odot .0019 * \operatorname{tet} a+\odot .363) *$ TF6 $-\operatorname{statev}(2 \odot)$

if (d6.lt.statev(3)) then

d6 $=$ statev $(3)$

end if

if $((\mathrm{d} 6 . g \mathrm{t} .0 .99)$.or.(Ed.ge.Ed_rup $))$ then

$\mathrm{d} 6=0.99$

$\mathrm{d} 2=\odot .99$

end if

statev $(6)=10.0$

end if

if (stress(2).1t.0.๑) then

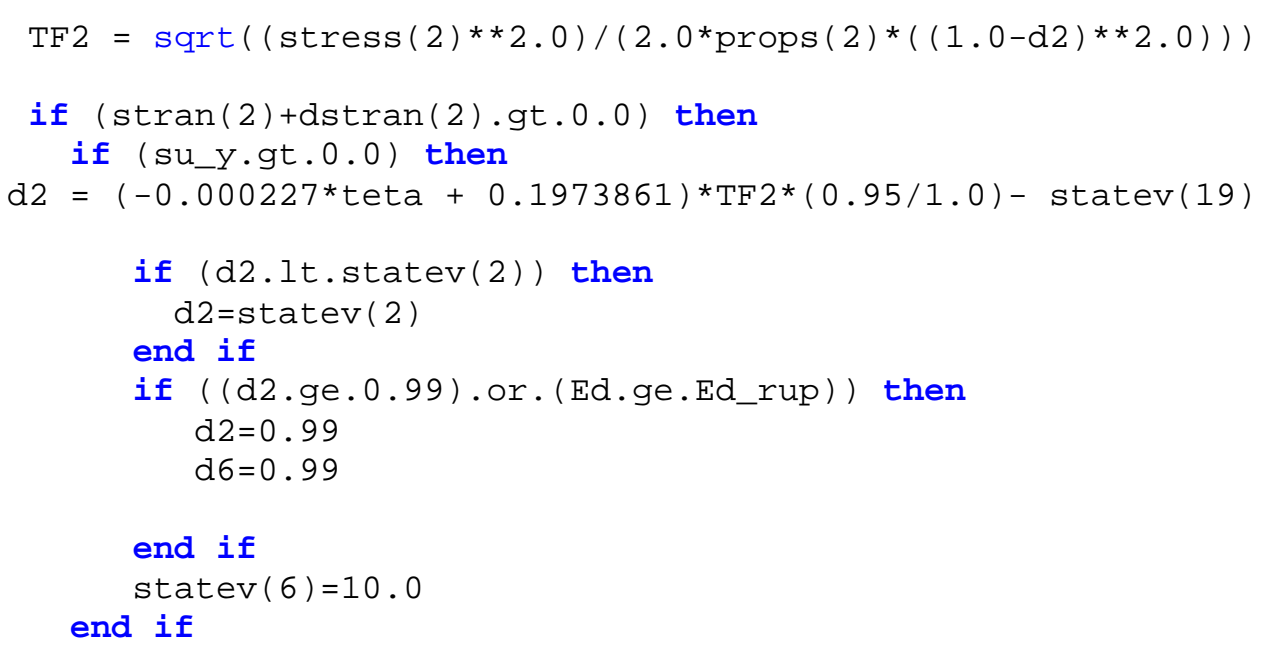


else if $(((\operatorname{stran}(2)+d s \operatorname{tran}(2)) \cdot l t . \odot . \Theta)$. and .

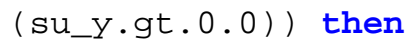

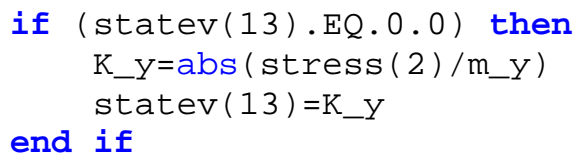

1 $\left.(1.0-\odot .09258)+\odot .09258^{*} \operatorname{props}(2)\right)$

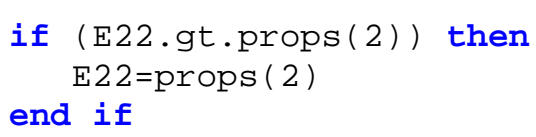

end if

end if

if (d2.lt.statev(2)) then

end if $\mathrm{d} 2=\operatorname{statev}(2)$

if (d6.lt.statev(3)) then d6=statev (3)

end if

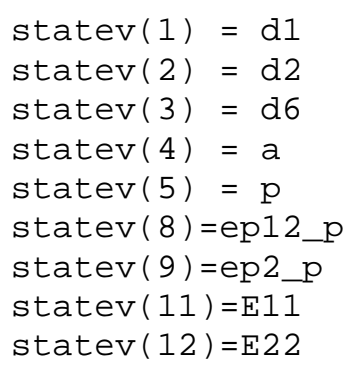

\section{RETURN}




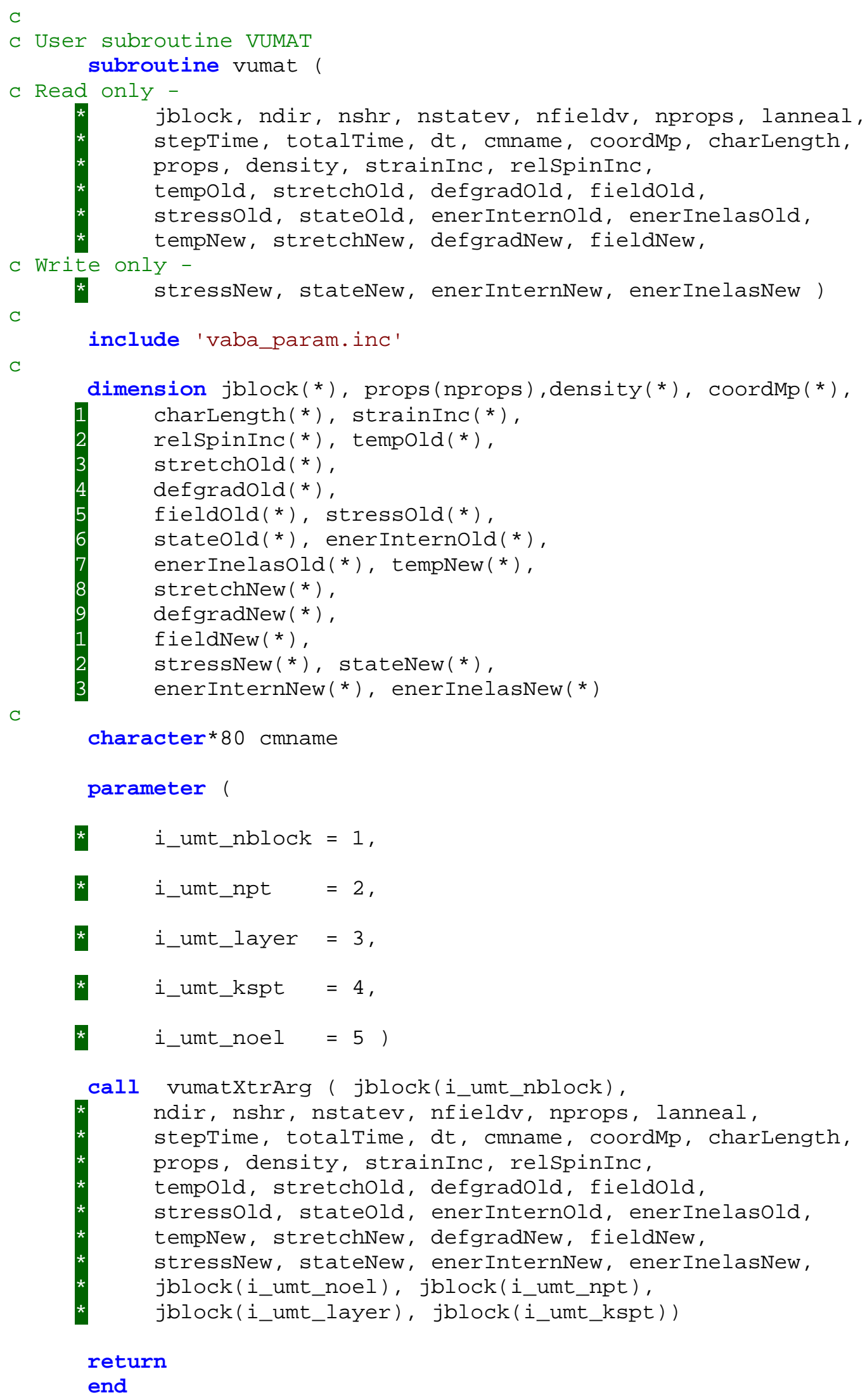

call vumatxtrArg ( jblock(i_umt_nblock), ndir, nshr, nstatev, nfieldv, nprops, lanneal, stepTime, totalTime, dt, cmname, coordMp, charLength, props, density, strainInc, relspinInc, tempold, stretchold, defgradold, fieldold, stressold, stateold, enerInternold, enerInelasold, tempNew, stretchNew, defgradNew, fieldNew, stressNew, stateNew, enerInternNew, enerInelasNew, jblock(i_umt_noel), jblock(i_umt_npt), jblock(i_umt_layer), jblock(i_umt_kspt)) 


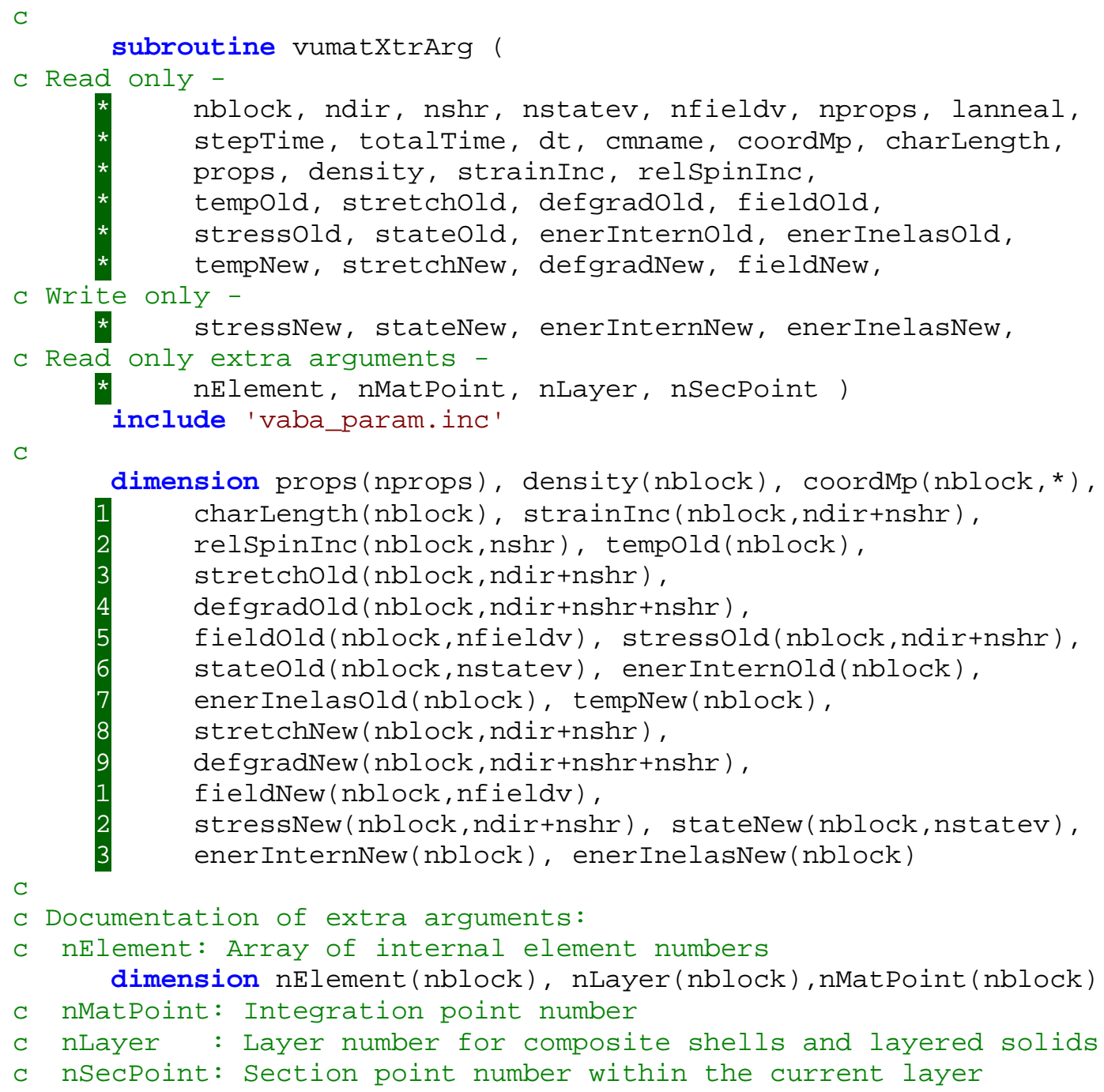

character 80 cmname

double precision E11, E22, nu12, G12, nu21, Xt, Xc, d1, TF2, d2,

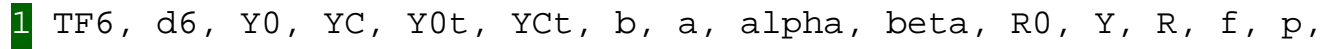
point_a, point_b, Y2C, starin1, teta, PS, AN, S12, E11r,E22r, E11c, E22c, d2_0, d6_0

integer flag

parameter $(X t=X X, X C=X X, Y \odot=\odot . \odot 9, Y C=2.07$,

$1 \mathrm{Y} \odot \mathrm{t}=\odot . \odot 92, \mathrm{YCt}=29.2, \mathrm{R} \odot=15 . \odot$, epsilon $\odot=\odot . \odot \odot 3, \mathrm{~S} 12=53 . \odot * 10 * * 6$, $2 \mathrm{Y} 2 \mathrm{~T}=0.3)$

C $\quad 314.0$

parameter $(b=1.02$, alpha=1.0, beta=3. $0, n p=10, a=0.5)$

dimension orientation(np), $\operatorname{strain}(3), \operatorname{ddsdde}(3,3)$

C Variables for read input file in order to assemble the orientation

C layer matrix

integer point, nc, ply, cp

real(8) ori, thick, gamma1, gamma2, gamma3, gamma, dfdt1, dfdt2,

1 dfdt3, ep1a, ep1b, dep1, DP, ef, ep2_p, ep12_p

c Vari veis para energia

double precision Ed, Ed_rup

parameter (Ed_rup=XX)

C Variables for matrix damage under compression

double precision $m_{-} f, m_{-} y$, su_y, su_f, S12_f, S12_y, K_y 
parameter $\left(m_{-} f=X X, m_{-} y=X X\right.$,

$\left.1 \mathrm{~S} 12 \_f=X X, S 12 \_y=X X\right)$

C State variables list

c state 1 - d1

c state $2-\mathrm{d} 2$

c state 3 - d6

c state 4 - E11

c state 5 - E22

c state 6 - flag

c state 7 - k_y

c state 8 - d2_0

c state $9-\mathrm{d} 6$ - 0

c state 10 - strain11

c steate 11 - strain22

C $\operatorname{parameter}($ job $=$ '3BP०90.inp', part $=$ 'composite')

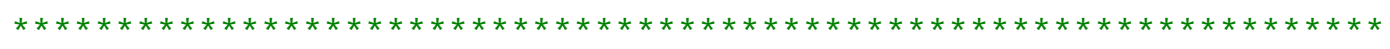

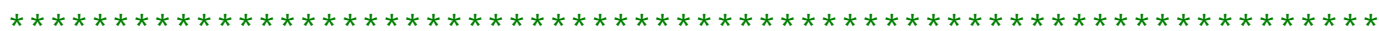

MATERIAL PROPERTIES

C MATERIAL

C

$\mathrm{E} 11=\operatorname{props}(1)$

$\mathrm{E} 22=\operatorname{props}(2)$

nu12 $=\operatorname{props}(3)$

G12 = props (4)

nu21 $=(\operatorname{props}(2) / \operatorname{props}(1)) * \operatorname{props}(3)$

C

do $100 \mathrm{~km}=1$, nblock

C PLY ORIENTATION

orientation $(1)=90 . \odot$

orientation $(2)=60.0$

orientation $(3)=-60.0$

orientation $(4)=90.0$

orientation $(5)=60.0$

orientation $(6)=-60.0$

orientation $(7)=90.0$

orientation $(8)=90.0$

orientation $(9)=-60.0$

orientation $(10)=60.0$

orientation $(11)=90.0$

orientation $(12)=-60.0$

orientation $(13)=60.0$

orientation $(14)=90.0$

teta=abs (orientation (nLayer(1)))

C

C Inicialization of variables

if ( $\operatorname{state0ld}(\mathrm{km}, 6) . \mathrm{lt} .0)$ then

stateold $(\mathrm{km}, 6)=0$

end if 


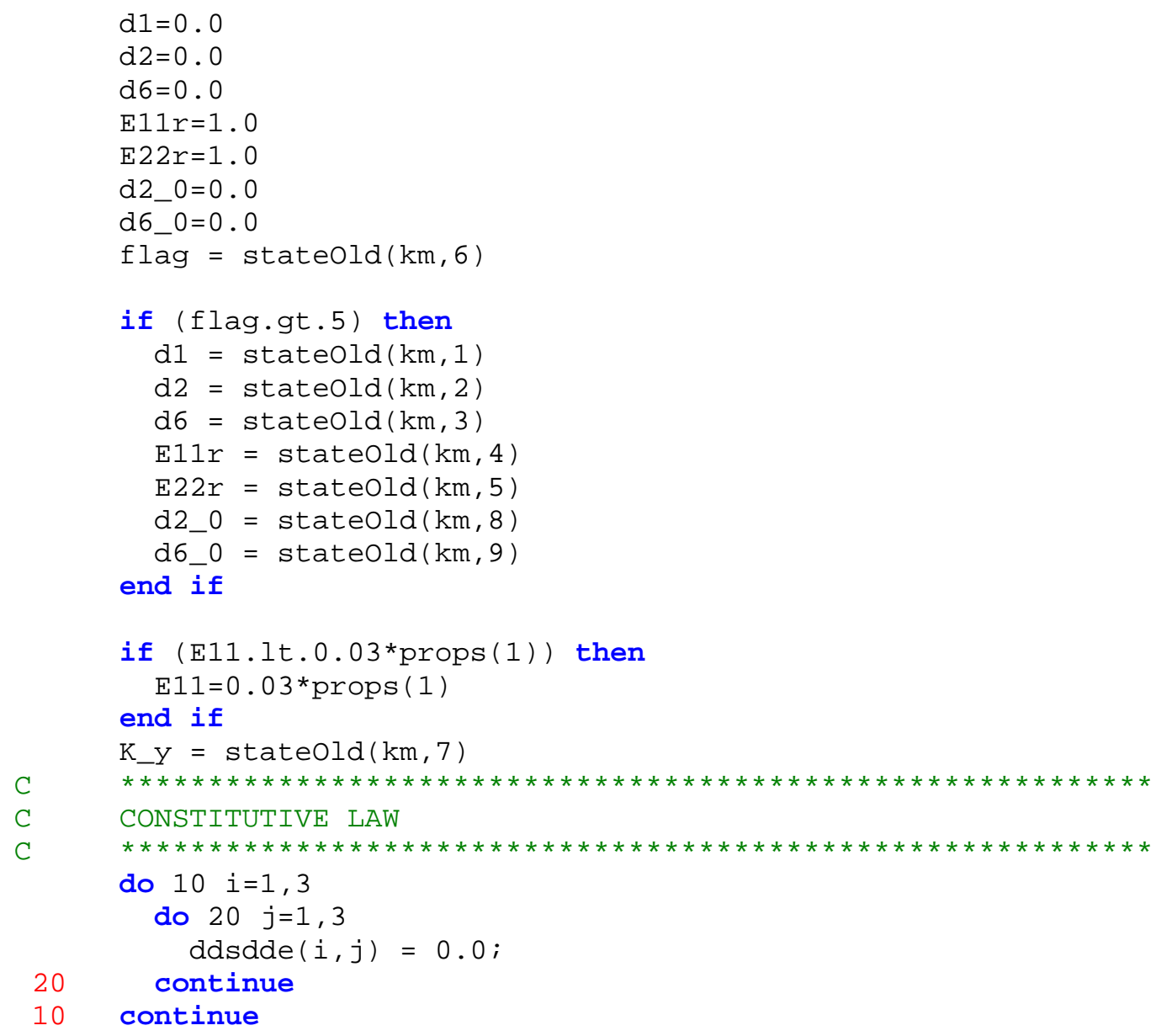

if ((nElement (km) . EQ. 883). and. (nMatPoint (km) .EQ.1)

1 . and. $(\mathrm{d} 2 . \mathrm{ne} . \odot . \odot))$ then write $\left({ }^{*},{ }^{*}\right) \mathrm{d} 2$, d2_0, nMatPoint $(\mathrm{km})$ end if

ddsdde $(1,1)=\left(E 1{ }^{*} E 11 r^{*}(1.0-d 1)\right) /(1.0-($ nu12* nu21)* $(1.0-d 2) *$

$1(1.0-\mathrm{d} 1))$

ddsdde $(2,2)=\left(E 2{ }^{*} E 22 r *(1 . \odot-d 2)\right) /(1 . \odot-(n u 12 * n u 21) *(1 . \odot-d 2) *$

$1(1.0-\mathrm{d} 1))$

ddsdde $(2,1)=\left((1 . \odot-d 2){ }^{*} n u 21 * E 1{ }^{*} E 11 r^{*}(1 . \odot-d 1)\right) /\left(1 . \odot-\left(n u 12{ }^{*} n u 21\right) *\right.$

$1(1.0-\mathrm{d} 2) *(1.0-\mathrm{d} 1))$

ddsdde $(1,2)=\operatorname{ddsdde}(2,1)$

$\operatorname{ddsdde}(3,3)=G 12 *(1 \cdot 0-\mathrm{d} 6)$

C Stresses calculations

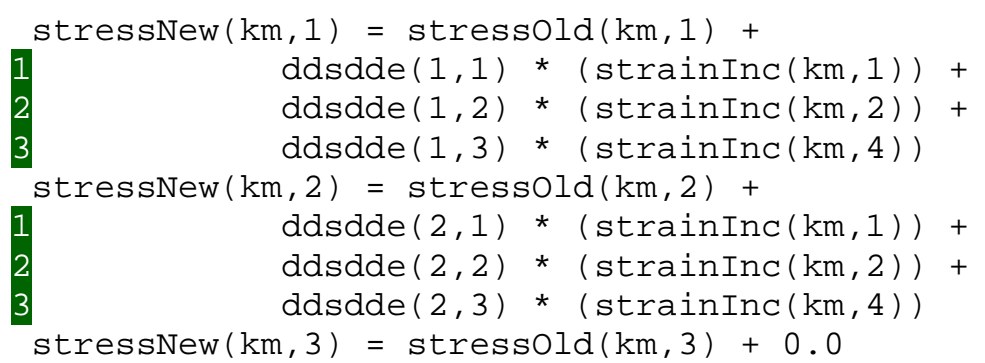




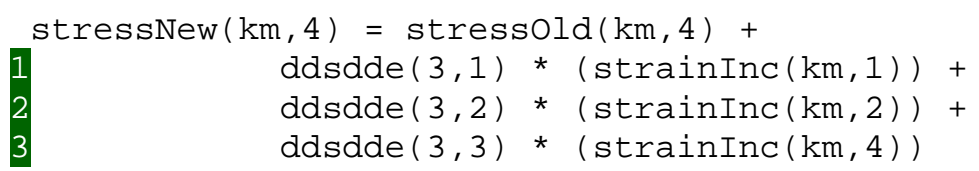

C strains

$\operatorname{strain}(1)=\operatorname{state0ld}(\mathrm{km}, 10)+\operatorname{strainInc}(\mathrm{km}, 1)$

$\operatorname{strain}(2)=\operatorname{state0ld}(\mathrm{km}, 11)+\operatorname{strainInc}(\mathrm{km}, 2)$

$\operatorname{strain}(3)=\operatorname{state0ld}(\mathrm{km}, 12)+\operatorname{strainInc}(\mathrm{km}, 4)$

Herakovich CAP. 9 - Damage Mechanics

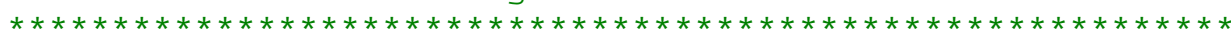

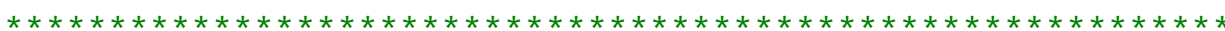

Strain Energy

if ( $\operatorname{stressNew}(\mathrm{km}, 2) \cdot \mathrm{gt} . \odot . \Theta)$ then

$\mathrm{Ed}=0.5^{*}(\operatorname{abs}(\operatorname{stressNew}(\mathrm{km}, 2) *(\operatorname{strain}(2)))+$

$1 \operatorname{abs}(\operatorname{stressNew}(\mathrm{km}, 4) *(\operatorname{strain}(3))))$

else if (stressNew $(k m, 2) \cdot l t .0 .0)$ then

$\mathrm{Ed}=0.5^{*}(\operatorname{abs}(\operatorname{stressNew}(\mathrm{km}, 2) *(\operatorname{strain}(2)))+$

$1 \operatorname{abs}(\operatorname{stressNew}(\mathrm{km}, 4) *(\operatorname{strain}(3))))$

end if

C Fiber failure - Max tensile or Max Compression

if $(\operatorname{stressNew}(k m, 1) \cdot g t \cdot 0 . \Theta)$ then

$f f=((\operatorname{stressNew}(k m, 1) / X t) * * 2 . \odot)$

if (ff.ge.1.0) then

$\mathrm{d} 1=\odot .99$

if ( $11.1 \mathrm{t}$. stateold $(\mathrm{km}, 1))$ then $\mathrm{d} 1=\operatorname{state} 0 \mathrm{ld}(\mathrm{km}, 1)$

end if

$\mathrm{flag}=10$

end if

else if (stressNew $(k m, 1) \cdot 1 \mathrm{t} . \odot . \odot)$ then

$f f=(\operatorname{stressNew}(k m, 1) / X c) * * 2.0$

if (ff.ge.1.0) then

1
2
3

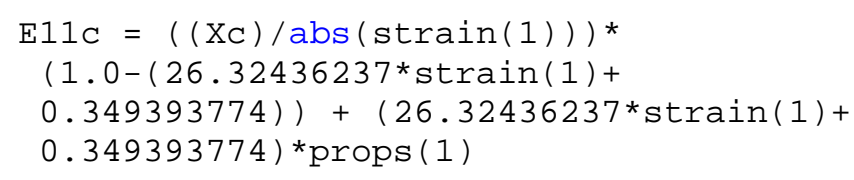

$\mathrm{E} 11 \mathrm{r}=\mathrm{E} 11 \mathrm{c} / \mathrm{props}(1)$

if (E11r.gt.1.0) then

$E 11 r=1.0$

end if 


$$
\mathrm{Flag}=10
$$

end if

end if

C Matrix damage

su_y $=(\operatorname{sqrt}(((\operatorname{stressNew}(\mathrm{km}, 2) * * 2.0)+(\operatorname{stressNew}(\mathrm{km}, 4) * * 2.0)))-$

$\left.1\left(-\mathrm{S} 12 \_\mathrm{y}+\left(\left(2 * \mathrm{~S} 12 \_\mathrm{y}\right) /\left(1+\left(\left(\operatorname{abs}(\operatorname{stressNew}(\mathrm{km}, 2)) / \mathrm{m} \_\mathrm{y}\right) * * 3.0\right)\right)\right)\right)\right)$

C Verification of failure criteria and evolution of plastic surface

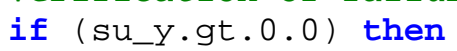

C Thermodynamic forces

if (stressNew $(\mathrm{km}, 2) \cdot g e \cdot \odot . \odot)$ then

$\mathrm{TF} 2=\operatorname{sqrt}((\operatorname{stressNew}(\mathrm{km}, 2) * * 2.0) /$

1 (2.0*props(2)))

else if (stressNew $(k m, 2) . l t .0 .0)$ then

$\mathrm{TF} 2=0.0$

end if

if (TF2.1t.10.0** -4.0$)$ then

$\mathrm{TF} 2=0.0$

end if

TF6 $=\operatorname{sqrt}((\operatorname{stressNew}(\mathrm{km}, 4) * * 2 . \odot) /$

$\left.1 \quad\left(2.0^{*} \operatorname{props}(4)\right)\right)$

if (TF6.lt.10.0**-4.0) then

$\mathrm{TF} 6=0.0$

end if

C Matriz tensile damage evolution parameter calculation

if ( (stateold $(\mathrm{km}, 8) \cdot \mathrm{EQ} \cdot \odot \cdot \odot)$. and .

1 (stressNew $(\mathrm{km}, 2) \cdot \mathrm{gt} . \odot . \Theta)$ ) then

d2_ $\odot=\left(-0.000215^{*}\right.$ teta +0.18752$) * T F 2$

end if

if (stateold $(\mathrm{km}, 9)$. EQ.๑.๑) then

end if

$d 6 \_0=(0.0019 *$ tet $a+0.363) *$ TF6

if $(($ su_y.gt.$\odot \cdot \odot)$. and. (stressNew $(\mathrm{km}, 2) \cdot \mathrm{gt} . \odot \cdot \odot)$. and .

$\left.1 \quad\left(\mathrm{~d} 2 \_0 . \mathrm{gt} . \Theta . \Theta\right)\right)$ then

$\mathrm{d} 2=(-\odot .000215 *$ teta +0.18752$) * T F 2-d 2 \_0$

if (d2.lt. . . $\odot)$ then $\mathrm{d} 2=\odot . \odot$

end if

if ( $\mathrm{d} 2.1 \mathrm{t}$.state0ld $(\mathrm{km}, 2))$ then

$\mathrm{d} 2=\operatorname{state0ld}(\mathrm{km}, 2)$ 
end if

if ((d2.ge.0.99).or.(Ed.ge.Ed_rup)) then

$\mathrm{d} 2=\odot .99$

end if

end if

$\mathrm{flag}=10$

C Matriz shear damage evolution parameter calculation

if (d6_0.gt.0.๑) then

$\mathrm{d} 6=(0.0019 *$ teta $+\odot .363){ }^{*}$ TF6 $-\mathrm{d} 6 \_0$

if (d6.1t.0.๑) then

end if

C $\quad$ stateNew $(\mathrm{km}, 3)=\mathrm{d} 6$

end if

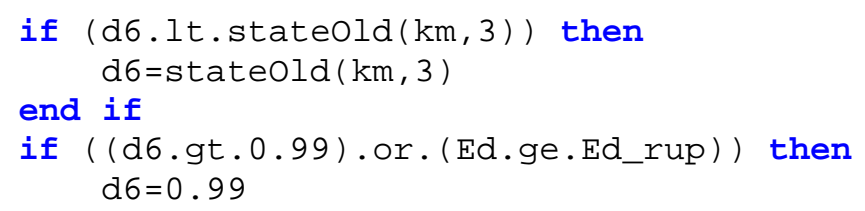

end if

flag $=10$

end if

if $\left(\left(\right.\right.$ su_y.gt $\left._{0} \odot \cdot \odot\right)$.and. $\left.(\operatorname{stressNew}(\mathrm{km}, 2) \cdot \mathrm{lt} \cdot \odot \cdot \odot)\right)$ then

1

$\operatorname{TF2}=\operatorname{sqrt}((\operatorname{stressNew}(\mathrm{km}, 2) * * 2.0) /$

(2.0*props(2)))

if (strain(2).gt. 0.0$)$ then

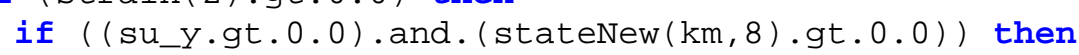

$\mathrm{d} 2=\left(-\odot .000215^{\star}\right.$ teta $\left.+\odot .18752\right){ }^{*} \mathrm{TF} 2-\mathrm{d} 2 \_0$

if $(\mathrm{d} 2 . \mathrm{tt} . \odot . \odot)$ then

$\mathrm{d} 2=0.0$

end if

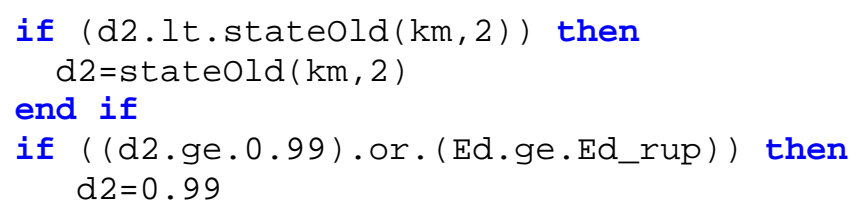


1

$(1.0-0.09258)+0.09258 * \operatorname{props}(2))$

$\mathrm{E} 22 \mathrm{r}=\mathrm{E} 22 \mathrm{c} / \mathrm{props}(2)$

if (E22r.gt.1.0) then

$E 22 r=1.0$

end if

if ((Ed.ge.Ed_rup)) then

$\mathrm{d} 2=0.99$

end if

$\mathrm{flag}=10$

end if

end if

if $(\mathrm{d} 2 . \mathrm{gt} .1 . \odot)$ then $\mathrm{d} 2=0.99$

end if

if (d6.gt.1.0) then $d 6=0.99$

end if

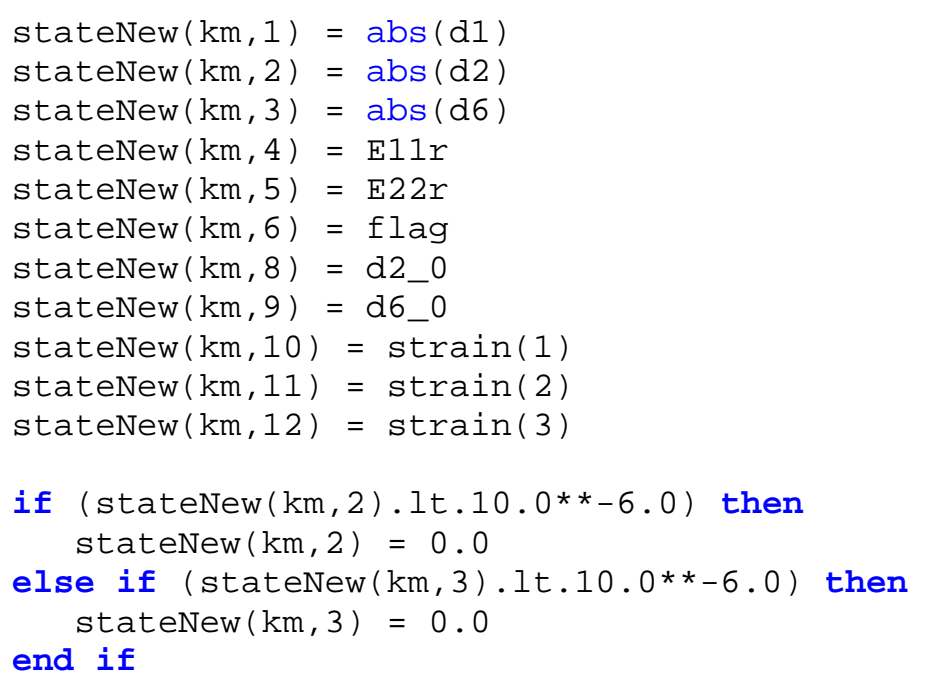

stateNew $(\mathrm{km}, 13)=\mathrm{TF} 2$

stateNew $(\mathrm{km}, 14)=\mathrm{TF} 6$

stateNew $(\mathrm{km}, 15)=$ su_y

stateNew $(\mathrm{km}, 16)=\mathrm{Ed}$

100 continue

RETURN

END

C 